\title{
Kleine und mittlere Unternehmen mit Besonderheiten? - Beiträge zur Mittelstandsforschung an den Beispielen von Innovation und Konjunktur
}

\author{
Dissertation \\ zur Erlangung des wirtschaftswissenschaftlichen Doktorgrades \\ der Wirtschaftswissenschaftlichen Fakultät \\ der Universität Göttingen
}

vorgelegt von

Dipl.-Volksw. Jörg Thomä

aus Ilmenau

Göttingen, 2012 
Erstgutachter: $\quad$ Herr Prof. Dr. Kilian Bizer

Zweitgutachter: $\quad$ Herr Prof. em. Dr. Wolfgang König

Tag der mündlichen Prüfung: 19.03.2012 


\section{Danksagung}

Verschiedene Personen haben zum Gelingen meiner Promotion beigetragen. Ihnen möchte ich daher an dieser Stelle meinen Dank aussprechen. Zu nennen ist insbesondere mein Doktorvater Herr Prof. Dr. Kilian Bizer, der mein Dissertationsvorhaben fortwährend gefördert und begleitet hat. Herrn Prof. Dr. Wolfgang König danke ich für seine wohlwollende Unterstützung und seinem regen Interesse an meinem gewählten Forschungsthema. Dank gebührt auch Herrn Prof. Dr. Wolfgang Benner für seine spontane Bereitschaft als Drittprüfer im Rahmen der Disputation zur Verfügung zu stehen.

Des Weiteren hat das angenehme Forschungsumfeld am Volkswirtschaftlichen Institut für Mittelstand und Handwerk an der Universität Göttingen (ifh) in vielerlei Hinsicht den notwendigen Rahmen für meine wissenschaftliche Arbeit geliefert. Hierfür möchte ich mich bei allen Kolleginnen und Kollegen herzlich bedanken. Daneben bin ich einer Reihe von weiteren Personen dankbar: Frau Dr. Margarita Tchouvakhina und Herrn Dr. Volker Zimmermann von der KfW Bankengruppe in Frankfurt am Main für die gewährte Möglichkeit zur Datennutzung und die ausgesprochen freundliche Betreuung während meines Forschungsaufenthaltes in ihrer Abteilung; dem Zentrum für Europäische Wirtschaftsforschung (ZEW) in Mannheim und namentlich meiner dortigen Ansprechpartnerin Frau Dr. Sandra Gottschalk ebenfalls für die Bereitstellung von Forschungsdaten; meinen Mitdoktorandinnen und -doktoranden für den gegenseitigen Austausch; und zu guter Letzt auch Herrn Dr. Joscha Beckmann für wichtige Hinweise und Anmerkungen.

Es ist für mich kaum vorstellbar, dass ich die Herausforderungen der Promotionszeit ohne die Unterstützung meiner Familie bewältigt hätte. Ein ganz besonderer Dank gilt daher meinen Eltern Elke und Reiner Thomä, weil sie mir jederzeit mit viel Rat und Tat zur Seite gestanden haben. Meine Frau Katja Thomä hat mich dabei mit ihrer liebevollen Art durch alle Höhen und Tiefen auf dem Weg zur Promotion begleitet und bestärkt. Ihr sei daher die vorliegende Arbeit gewidmet.

Göttingen, 04.04.2012

Jörg Thomä 


\section{Inhaltsverzeichnis / Contents}

\section{Kapitel I}

Einführung und Zusammenfassung der Einzelbeiträge

\section{Kapitel II}

Kleinunternehmen und der Schutz von Innovationen - benachteiligt oder einfach anders?

Eingereicht bei „Zeitschrift für KMU und Entrepreneurship (ZfKE)“ im November 2009, Angenommen im Januar 2010, Veröffentlicht im April 2010, Jahrgang 57, Heft 3-4, S. 219-238, DOI: 10.3790/zfke.57.3-4.219.

\section{Kapitel III}

To protect or not to protect? Modes of appropriability in the small enterprise sector (gemeinsam mit Kilian Bizer; Hauptautor: Jörg Thomä)

Working Paper

\section{Kapitel IV}

Innovation protection practices in small and medium enterprises (SMEs)

(gemeinsam mit Volker Zimmermann; Hauptautor: Jörg Thomä)

Working Paper

\section{Kapitel V}

Das Handwerk - ein Konjunkturstabilisator?

Veröffentlicht in: Küpper, H.-U. (Hrsg.), Lehren aus der Krise. Auswirkungen und Konsequenzen aus der Finanz- und Wirtschaftskrise 2008/2009 für den Handwerksbereich, München 2010, ISBN: 978-3-925397-61-5, S. 19-75.

Veröffentlicht in zusammengefasster Form: Thomä, J. (2011): Das Handwerk als Stabilisator der konjunkturellen Entwicklung?, in: Wirtschaftsdienst - Zeitschrift für Wirtschaftspolitik, Jg. 91, Heft 2, S. 127-132, DOI: 10.1007/s10273-0111193-y. 


\section{Weitere Veröffentlichungen, die nicht Teil dieser Dissertation sind}

\section{Veröffentlichungen mit externem wissenschaftlichen Begutachtungsverfahren:}

Fiskalische Wirkungsanalysen zur Bewertung der Siedlungsentwicklung - Ein (Fehl-)Versuch zur Flächenverbrauchsreduktion? (gemeinsam mit Ralph Henger)

Eingereicht bei „Zeitschrift für angewandte Umweltforschung (ZAU)“ im März 2009, Angenommen im Mai 2009, Veröffentlicht im Dezember 2009, Jg. 19, Heft 1-2, S. 58-77.

\section{Sonstige Veröffentlichungen:}

Thomä, J. und Zimmermann, V. (2012), Innovationsschutz im Mittelstand: Strategien und deren Bestimmungsfaktoren, in: KfW Research, Reihe „Standpunkt“ Nr. 16, März 2012.

Thomä, J. (2009): Wohnzufriedenheit und Nutzerpräferenzen im Quartier - Ergebnisse zweier Bewohnerbefragungen, in: Land Use Economics and Planning, Discussion Paper No. 09-06.

Thomä, J. und Krüger, L. (2009): Perspektiven der Gesetzesfolgenabschätzung in der Anwendung, Tagungsbericht zu einem Symposium der Sonderforschungsgruppe Institutionenanalyse, in: Zeitschrift für Politikwissenschaft (ZPol), Jg. 19, Heft 1, S. 157-164.

Thomä, J. (2009): Nachfrageorientiertes Nutzungszyklusmanagement von Wohnquartieren aus ökonomischer Perspektive, in: Land Use Economics and Planning, Discussion Paper No. 08-03. 


\section{Kapitel I}

Einführung und Zusammenfassung der Einzelbeiträge 


\section{Inhalt}

1. Kleine und mittlere Unternehmen als Forschungsgegenstand 9

2. Einführung in den Themenzusammenhang $\quad 12$

2.1 Innovation und Unternehmensgröße 12

2.2 Konjunktur und Unternehmensgröße 14

2.3 Überblick zu den Einzelbeiträgen der Dissertation 17

3. Zusammenfassung der Einzelbeiträge: Hintergrund, Ergebnisse, Implikationen 21

3.1 Kleinunternehmen und der Schutz von Innovationen - benachteiligt oder einfach anders? $\quad 21$

3.2 To protect or not to protect? Modes of appropriability in the small enterprise sector 24

3.3 Innovation protection practices in small and medium enterprises (SMEs) 28

3.4 Das Handwerk - ein Konjunkturstabilisator? 30

$\begin{array}{ll}\text { Literaturverzeichnis } & 33\end{array}$ 


\section{Kleine und mittlere Unternehmen als Forschungsgegenstand}

"The differences in the administrative structure of the very small and the very large firms are so great that in many ways it is hard to see that the two species are of the same genus. [...]; we cannot define a caterpillar and then use the same definition for a butterfly."1

Kleine und mittlere Unternehmen (KMU) gelten in der wirtschaftspolitischen Diskussion als Garanten für Beschäftigung und Wettbewerbsfähigkeit. Damit diese ihr Entwicklungs- und Innovationspotenzial voll ausschöpfen können, bildet aktuell die Schaffung optimaler Rahmenbedingungen für KMU den Ansatzpunkt für eine Reihe politischer Maßnahmen sowohl auf nationaler als auch auf europäischer Ebene. ${ }^{2}$ Trotz der in der Praxis mitunter abweichenden Zielvorgaben und Schwerpunktsetzungen der einzelstaatlichen Programme und Initiativen wird hierbei in der Regel im Kern übereinstimmend vorausgesetzt, dass KMU aufgrund verschiedener größenbedingter Benachteiligungen einer besonderen Unterstützung bedürfen. Die annahmegemäß mit der Behebung eines entsprechenden Marktversagens ${ }^{3}$ verbundene Nutzensteigerung liefert somit eine mögliche Rechtfertigungsgrundlage für die Existenz einer KMU-orientierten Wirtschaftspolitik. Ein systematisches Marktversagen im KMU-Sektor liegt jedoch keineswegs automatisch vor, sind mit einer kleineren Unternehmensgröße doch neben Nachteilen stets auch potenzielle Vorteile verbunden. Zudem ist im konkreten Fall jeweils abzuwägen, ob der staatliche Eingriff insgesamt zu einer Wohlfahrtsverbesserung führt, also die hiermit erzielte Nutzensteigerung dessen Kosten überwiegt und keine effizientere Handlungsalternative mit denselben Resultaten existiert. Politische Entscheidungsträger stehen daher vor der grundsätzlichen Herausforderung, zur notwendigen Begründung ihrer KMU-spezifischen Maßnahmen deren Ausrichtung und den letztlichen Zielerreichungsgrad nicht zuletzt mit Blick auf den jeweiligen Marktversagenstatbestand kontinuierlich z.B. mit Hilfe von Programmevaluierungen zu hinterfragen. ${ }^{4}$

Nach Curran und Blackburn (2001), S. 162f, kann auch die Mittelstandsforschung, verstanden als empirischer Ansatz zur Untersuchung von kleineren Unternehmen und ihrer möglichen Besonderheiten, einen wichtigen Beitrag dazu leisten, das für eine effektive Ausgestaltung politischer Unterstützungsmaßnahmen benötigte Wissen hinsichtlich der Lage, Entwicklung und Problemfelder von KMU zu erhöhen. Ein theoretisch idealer Ausgangspunkt entsprechen-

$1 \quad$ Penrose, E. (1959), S. 19.

2 Vgl. z.B. European Commission (2008) und BMWi (2011).

3 Einen Überblick mit einer Reihe von Beispielen bezüglich möglicher KMU-spezifischer Marktversagenstatbestände, die etwa durch das Vorliegen von asymmetrischer Information oder positiver Externalitäten verursacht werden können, liefern Bridge, S. u. a. (2009), S. 337ff.

4 Vgl. Storey, D. J. (1994), S. 253ff; Storey, D. J. (2005), S. 475ff; Bridge, S. u. a. (2009), S. 329ff; Scheidegger, E. und Brunetti, A. (2003). Die normative Annahme einer am Gemeinwohl orientierten KMUPolitik kann freilich die Existenz und die Ausgestaltung entsprechender Maßnahmen nicht vollständig erklären. Aus politökonomischer Sicht (,public choice“) müssten in diesem Zusammenhang z.B. auch die eigennutzorientierten Kalküle von Politikern, die Budgetmaximierung der Bürokraten oder der Einfluss von Interessengruppen im politischen Entscheidungsprozess berücksichtigt werden. 
der Forschungsanstrengungen wäre dabei eine Definition, die durch qualitative Merkmale die Gesamtheit der KMU von Großunternehmen möglichst trennscharf abzugrenzen vermag. Diesbezüglich naheliegend ist z.B. die gerade in KMU häufig ausgeprägte und insbesondere dem traditionell in Deutschland stark verankerten Mittelstandsbegriff zu Grunde liegende enge Verbindung zwischen Eigentum und Leitung, die sich im idealtypischen Fall durch die Einheit der wirtschaftlichen Existenz des Inhabers und seines Unternehmens mit der daraus resultierenden persönlichen Verantwortlichkeit des Unternehmers zeigt. In der empirischen Forschung scheitert der Versuch einer solchen oder ähnlichen qualitativen KMU-Abgrenzung jedoch häufig an der großen Heterogenität kleinerer Unternehmen und der schwierigen Messbarkeit vor allem im Rahmen breiter empirisch-quantitativer Untersuchungen. ${ }^{5}$

Abbildung 1: KMU in Deutschland 2008 - Unternehmenszahl, Umsatz und Beschäftigte

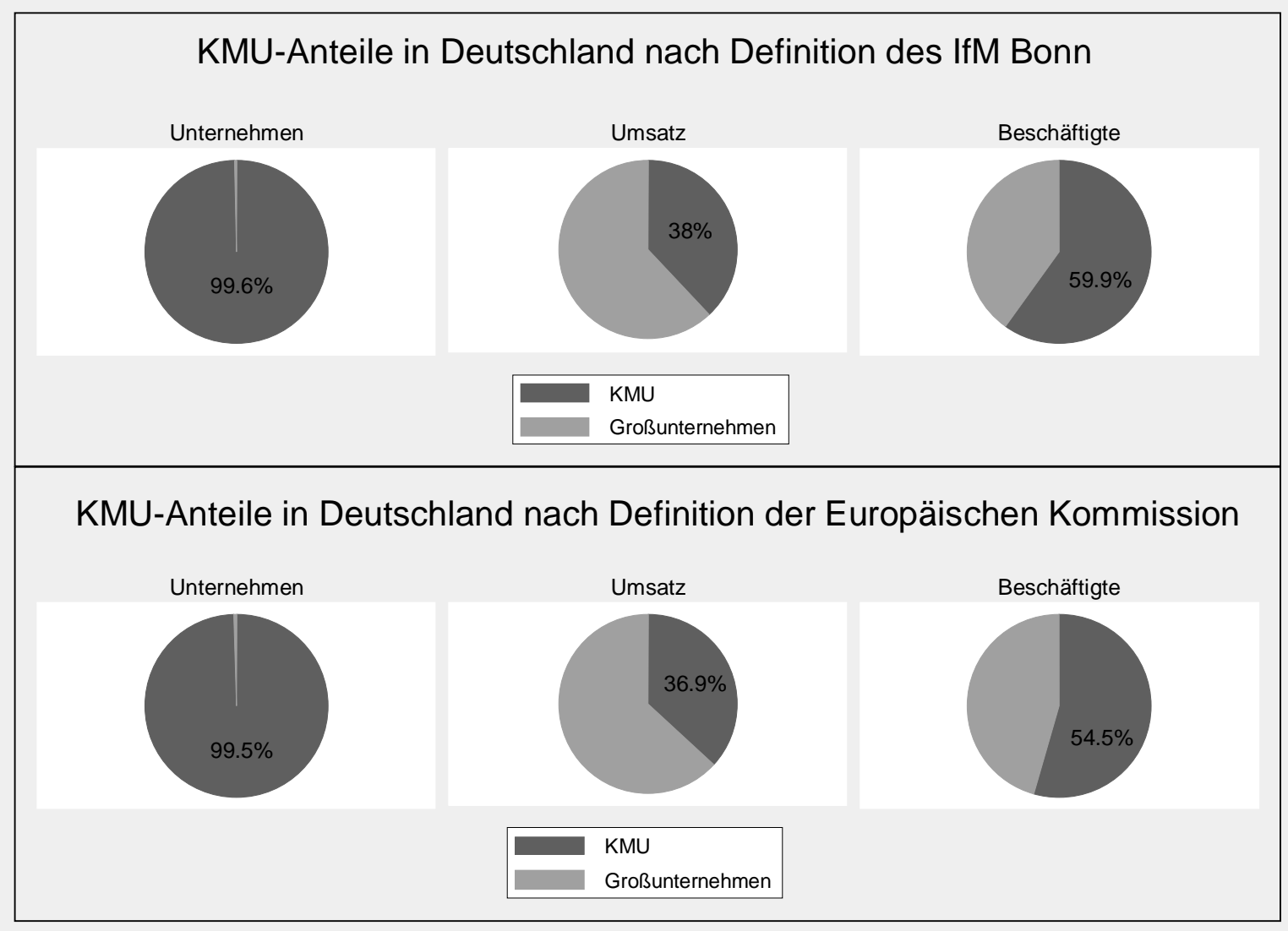

Quelle: Berechnungen des ifm Bonn auf Grundlage des Unternehmensregisters (Statistisches Bundesamt, Auswertungsstichtag: 30.06.2010), in: http://www.ifm-bonn.org (letzter Zugriff: 01.07.2011).

Zur besseren Operationalisierung von KMU haben sich daher in der Wissenschaft pragmatischere Abgrenzungen auf der Grundlage von quantitativen Kriterien durchgesetzt, obwohl mit einer solchen Vereinfachung zwangsläufig unternehmens- und branchenspezifische Merkmale nur eingeschränkt berücksichtigt werden können. ${ }^{6}$ So ist beispielsweise im deutschen

5 Vgl. Welter, F. (2003), S. 28f; Krämer, W. (2003), S. 8f. Für eine detaillierte Diskussion der Schwierigkeiten bei der Abgrenzung von KMU siehe Curran, J. und Blackburn, R. A. (2001), S. 8ff.

6 Vgl. Curran, J. und Blackburn, R. A. (2001), S. 9f; Welter, F. (2003), S. 29f; Krämer, W. (2003), S. 9ff. 
Sprachraum in Arbeiten aus dem Bereich der Mittelstandsforschung die Grenze von 499 Beschäftigten weit verbreitet, was z.B. durch die KMU-Definition des Bonner Instituts für Mittelstandsforschung (IfM Bonn) ${ }^{7}$ zum Ausdruck kommt. Im internationalen Kontext gewinnt demgegenüber die Obergrenze für KMU bei 249 Beschäftigten in Anlehnung an die KMUDefinition der Europäischen Kommission ${ }^{8}$, welche primär dem Zweck der wirtschaftspolitisch orientierten Festlegung der möglichen Inanspruchnahme von Fördermaßnahmen dient, auch für die Wissenschaft verstärkt an Relevanz. Unabhängig davon welche dieser beiden Beschäftigungsobergrenze in einer Forschungsarbeit letztlich zu Anwendung kommt, zeigt Abbildung 1 jedoch, dass - gemessen an den quantitativen Schlüsselgrößen Unternehmenszahl, Umsatz und Beschäftigung - den Unternehmen mit 250 bis zu 499 Beschäftigten nur ein relativ geringes Gewicht zukommt. Gleichzeitig wird anhand von Abbildung 1 zudem der volkswirtschaftliche Stellenwert von kleineren Unternehmen deutlich, wodurch allein deshalb eine KMU-Perspektive bei der Untersuchung von unternehmensbezogenen und gleichzeitig wirtschaftspolitisch relevanten Sachverhalten gerechtfertigt erscheint.

Der umrissene Ansatz der Mittelstandsforschung bildet den Kerngegenstand der vorliegenden Arbeit. Zur besseren Einordnung der diesbezüglichen Einzelbeiträge ${ }^{9}$ führt dieses Überblickskapitel in die jeweiligen Forschungsfragen ein. Ferner werden die entsprechenden Untersuchungsergebnisse zusammengefasst und die daraus resultierenden Implikationen diskutiert. Das Kapitel I gliedert sich dabei in zwei Abschnitte: Im Folgenden liefert Abschnitt 2 zunächst aus der KMU-Perspektive einen Literaturüberblick zu den beiden übergreifenden Themenbereichen der Dissertation. Hierdurch soll deutlich werden, dass insbesondere die Themenfelder Innovation und Konjunktur jeweils für sich genommen dazu geeignet sind, den charakteristischen Besonderheiten kleinerer Unternehmen nachzuspüren. Zur Einordnung in diesen Fachkontext erfolgt daraufhin eine überblicksartige Einführung in den Untersuchungsgegenstand der vier Einzelbeiträge. Vor dem Hintergrund der einschlägigen Literatur fasst Abschnitt 3 dann die einzelnen Beiträge zusammen und formuliert auf dieser Grundlage verschiedene wirtschaftspolitische Implikationen der Untersuchungsergebnisse. Mitunter fallen die Ausführungen hierbei inhaltlich detaillierter aus als in den entsprechenden Kapiteln der Dissertation. Dies geschieht, um die Zusammenhänge zwischen den in sich geschlossenen Einzelbeiträgen besser verdeutlichen zu können und um bestimmte Aspekte aus der übergreifenden Perspektive dieses Kapitels eingehender zu vertiefen.

7 Nach der KMU-Definition des IfM Bonn gelten unabhängige Unternehmen mit bis zu 9 Beschäftigten und weniger als 1 Mio. EUR Jahresumsatz als kleine Unternehmen und solche mit 10 bis 499 Beschäftigten und einem Jahresumsatz von bis unter 50 Mio. EUR als mittlere Unternehmen. Vgl. http://www.ifm-bonn.org.

8 Nach der KMU-Definition der Europäischen Kommission ergeben sich für die Gruppe der Kleinstunternehmen sowie der kleinen und mittleren Unternehmen (insgesamt: KMU) folgende Schwellenwerte: Kleinstunternehmen ( $<10$ Beschäftigte, Jahresumsatz und Jahresbilanzsumme $\leq 2$ Mio. EUR); kleine Unternehmen ( $<50$ Beschäftigte, Jahresumsatz und Jahresbilanzsumme $\leq 10$ Mio. EUR); mittlere Unternehmen ( $<250$ Beschäftigte, Jahresumsatz $\leq 50$ Mio. EUR, Jahresbilanzsumme $\leq 43$ Mio. EUR). Zudem muss ein KMU eigenständig sein, d.h. es ist entweder völlig unabhängig oder nicht über einen Kapital- bzw. Stimmrechtsanteil von über 25 \% mit anderen Unternehmen verbunden. Vgl. European Commission (2005).

$9 \quad$ Siehe Kapitel II bis V dieser Dissertation. 


\section{Einführung in den Themenzusammenhang}

\subsection{Innovation und Unternehmensgröße}

Im Falle des Themengebiets Innovation ${ }^{10}$ sind an dieser Stelle die Ergebnisse der in der Vergangenheit stark diskutierten Frage von besonderem Interesse, inwiefern in der Unternehmensgröße eine zentrale Determinante der Innovationstätigkeit gesehen werden kann. Hiermit

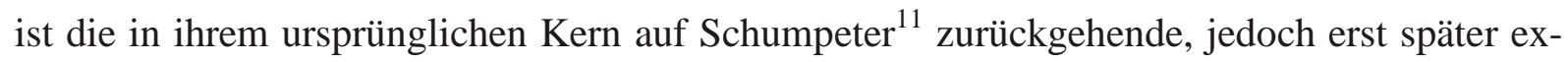
plizit ausformulierte Hypothese angesprochen, dass größere Unternehmen innovativer seien als kleinere. Nach Cohen, W. (1995), S. 184f, lassen sich für eine solche Vermutung in der Tat verschiedene Begründungen anführen. Demzufolge wird z.B. argumentiert, dass Großunternehmen vor allem deshalb über einen ausgeprägten Vorteil im Rahmen der Innovationstätigkeit verfügen, da sie im ressourcenintensiven Bereich der Forschung \& Entwicklung (F\&E) ihre Größen- und Verbundvorteile effektiv ausspielen können, über bessere Möglichkeiten zur Finanzierung entsprechender Vorhaben verfügen und auch eher in der Lage sind, die wirtschaftlichen Risiken ihrer Innovationstätigkeiten durch die gleichzeitige Durchführung von verschiedenen F\&E-Projekten zu reduzieren (Risikodiversifizierung). Eine umfangreiche Zahl an vorliegenden Arbeiten hat in der Schumpeter'schen Tradition den Zusammenhang zwischen einer F\&E-Tätigkeit - verstanden als zentraler Inputfaktor für Innovationen und der Unternehmensgröße untersucht. Als übereinstimmendes Ergebnis wurde hierbei in der Tat ein positiver Zusammenhang zwischen diesen beiden Größen festgestellt. ${ }^{12}$

Aus zwei Gründen führt jedoch eine alleinige Orientierung an F\&E-bezogenen Indikatoren zur Unterschätzung des Innovationspotenzials von KMU. Erstens hat F\&E in kleineren Unternehmen einen informelleren und weniger institutionalisierten Charakter als in größeren

10 Im Rahmen der vorliegenden Dissertation orientiert sich der Begriff Innovation an den Definitionen und Standards des Oslo-Manual. Demnach wird unter einer Produktinnovation ein neues oder merklich verbessertes Produkt (inkl. Dienstleistungen) und unter einer Prozessinnovation eine neue oder merklich verbesserte Fertigungs- / Verfahrenstechnik bzw. ein neues oder merklich verbessertes Verfahren zur Dienstleistungserbringung / zum Produktvertrieb verstanden. Für die Einschätzung des Neuheitsgrades ist dabei jeweils die Unternehmenssicht ausschlaggebend. Im Detail siehe OECD und Eurostat (2005), S. 45ff.

11 In seiner Theorie der wirtschaftlichen Entwicklung hatte Schumpeter noch als Beispiel für die Erfüllung der zur Erbringung von Innovationen („Durchsetzung neuer Kombinationen“) von ihm als zentral betrachteten Unternehmerfunktion unter anderem den „Gründer“ genannt und somit indirekt auf die dynamische Rolle kleinerer Unternehmen verwiesen (Vgl. Schumpeter, J. A. (1934), S. 100ff und S. 115f). Im Rahmen seines späteren Werks Kapitalismus, Sozialismus und Demokratie sah Schumpeter jedoch zunächst kleine und mittlere Unternehmen und zuletzt auch die Unternehmerfunktion selbst durch das unaufhaltsame Vordringen von Großunternehmen, die als „vollkommen bürokratisierte industrielle Rieseneinheiten“ mit ihren „geschulten Spezialistengruppen“ zunehmend stärker den technischen Fortschritt und damit letztlich die wirtschaftliche Entwicklung an sich bestimmen würden, in der zwangsläufigen Verdrängung begriffen (Vgl. Schumpeter, J. A. (1942), S. 174f und S. 213ff).

12 Für umfangreiche Literaturübersichten siehe Acs, Z. J. und Audretsch, D. B. (2005), S. 62ff; Cohen, W. (1995), S. 185ff und Symeonidis, G. (1996), S. 6ff. Es herrscht jedoch weitgehend Einigkeit darüber, dass das Ausmaß von F\&E-Aktivitäten typischerweise nur proportional mit der Unternehmensgröße wächst. Zudem zeigte sich ebenfalls übereinstimmend, dass kleinere Unternehmen tendenziell eine größere F\&E-Produktivität (d.h. die Anzahl erbrachter Innovationen im Verhältnis zum F\&E-Input) aufweisen. 
Unternehmen, da oftmals weder eine formale F\&E-Abteilung noch ein explizit ausgewiesenes F\&E-Budget vorhanden sind. Zudem liegt der Schwerpunkt hierbei anstatt auf systematischen Forschungsanstrengungen (dem „, $\mathrm{F}^{“}$ ) eher auf marktnahen Entwicklungen (dem „Е“), bei denen vor allem personenbezogenes Erfahrungswissen und Können als Inputfaktor für Innovationen im Vordergrund stehen. Quantifizierbare Messgrößen wie die absolute Höhe der F\&EAusgaben können daher das Innovationspotenzial entsprechender KMU-Aktivitäten lediglich unvollständig erfassen. Dieser Umstand gilt umso mehr, da kleinere Unternehmen im operativen Tagesgeschäft eher flexibel auf die nachfrageseitige Notwendigkeit zur ressourcenzehrenden F\&E-Aktivität reagieren und eine solche daher anstatt auf regulärer oftmals nur auf gelegentlicher Basis zur Umsetzung von konkreten Problemlösungen betreiben. ${ }^{13}$ Zweitens sind kleinere Unternehmen häufig auch ohne ein F\&E-intensives Engagement dazu in der Lage, relevante Innovationsbeiträge zu leisten. Die Art des Neuheitsgrades von KMU-Innovationen bestimmt sich in vielen Fällen daher gerade nicht durch die Einführung von grundlegend neuen Produkten und Dienstleistungen. Stattdessen ist vielmehr die Fähigkeit kleinerer Unternehmen entscheidend, individuellen Kundenbedürfnissen z.B. hinsichtlich der Qualitätseigenschaften von bereits existierenden Produkten durch inkrementelle Anpassungs- und Verbesserungsleistungen schnell und flexibel entgegenzukommen und ein solches Angebot im Idealfall noch durch produktbegleitende Dienstleistungen ergänzen zu können. Innovative KMU sind dabei häufig in Marktnischen zu finden und können mit Hilfe einer solchen, auf die Erreichung von Qualitätsvorsprüngen abzielenden Differenzierungsstrategie ihre Wettbewerbsfähigkeit sichern. $^{14}$

Nur weil Innovationsprozesse in kleineren Unternehmen weniger durch systematische F\&EAnstrengungen geprägt sind, bedeutet dies folglich nicht gleichzeitig, dass KMU weniger innovativ wären als Großunternehmen. Aus theoretischer Sicht sind in diesem Zusammenhang vielmehr die Komplementaritäten zwischen kleineren und größeren Unternehmen hervorzuheben, da diese im idealtypischen Fall auf Grundlage ihrer jeweiligen Stärken und Schwächen sich ergänzende und daher gleichsam wichtige Innovationsbeiträge leisten. ${ }^{15}$ Die klassische Zusammenstellung von Rothwell (1983), S. 16, zu den Vor- und Nachteilen, die eine kleinere Unternehmensgröße im Rahmen der Innovationstätigkeit oftmals mit sich bringt, liefert diesbezüglich einen ersten Überblick. Demnach sind kleinere Unternehmen bei der Erbringung von Innovationen aufgrund ihrer kleineren Ressourcenbasis vor allem unter materiellen Gesichtspunkten im Nachteil. Sie würden jedoch über verhaltensbezogene Vorteile (Kundennähe, Flexibilität, effiziente interne Entscheidungswege etc.) verfügen, die in dieser Form durch größere Konkurrenten nur schwer nachzuahmen sind. Ein solches Schema kann freilich nur ein erster Ansatzpunkt zur Untersuchung des Innovationsverhaltens von kleineren Unternehmen sein. Denn vor allem bei innovativen KMU handelt es sich um eine sehr hetero-

13 Vgl. Kleinknecht, A. H. (1987); Kleinknecht, A. H. (1991); Baldwin, J. und Gellatly, G. (2003), S. 67f; Lahner, J. (2004), S. 103f.

14 Vgl. Wynarczyk, P. u. a. (1993), S. 30ff; Baldwin, J. und Gellatly, G. (2003), S. 68; Mazzarol, T. und Reboud, S. (2009), S. 213f; Pleschak, F. u. a. (1994), S. 14f.

15 Vgl. Nooteboom, B. (1994), S. 335f; Lahner, J. (2004), S. $194 f$. 
gene Gruppierung, bei der die Spannweite vom mittelständischen Hightech-Unternehmen bis zum traditionellen Handwerksbetrieb reicht. Hierdurch ergibt sich die Relevanz einer differenzierten Forschungsperspektive, die den spezifischen Eigenschaften bestimmter Typen von KMU und den jeweils charakteristischen Merkmalen ihrer Innovationsbeiträge Rechnung trägt. ${ }^{16}$ Entsprechend ausgerichtete Studien ordnen sich dabei in eine allgemeine Entwicklungslinie der Innovationsforschung ein, wonach zur Erklärung der beobachtbaren Heterogenität im Innovationsgeschehen anstelle der Unternehmensgröße vor allem Einflussfaktoren wie das Vorhandensein von technologischen Möglichkeiten, Nachfragebedingungen, Eigenschaften der relevanten Wissensbasis oder die Aussicht auf eine erfolgreiche Aneignung von Erträgen aus der eigenen Innovationstätigkeit heranzuziehen sind. ${ }^{17}$

\subsection{Konjunktur und Unternehmensgröße}

Im Vergleich zum vorrangegangenen Themenbereich wurde der Zusammenhang zwischen dem Verlauf der Konjunktur ${ }^{18}$ und der Rolle der Unternehmensgröße im Rahmen von empirischen Studien - wohl nicht zuletzt aufgrund der schwierigen Datenlage - deutlich seltener untersucht. Dabei mangelt es nicht an Einschätzungen hinsichtlich möglicher Besonderheiten von KMU im Konjunkturverlauf. In kleineren Unternehmen würden demnach die Auswirkungen von gesamtwirtschaftlichen Konjunkturschwankungen auf Output und Beschäftigung geringer ausfallen als in größeren Unternehmen, was für eine aus wirtschaftspolitischer Sicht wichtige Stabilisatorfunktion von KMU gerade in Zeiten rezessiver Phasen sprechen würde. Gemäß Krämer (2003), S. 26, lassen sich diesbezüglich zunächst verschiedene absatzorientierte Argumente anführen. Die Fokussierung der Mehrzahl der KMU auf den Binnenmarkt mit einer überwiegend lokalen und regionalen Absatzausrichtung würde z.B. dazu führen, dass kleinere Unternehmen im Durchschnitt weniger abhängig vom besonders volatilen Exportgeschäft sind. Dieser Umstand bildet in der Regel die Erklärungsgrundlage, warum

16 Dies gilt nicht zuletzt auch für wirtschaftspolitische Handlungserfordernisse, die sich hierdurch zielgenauer ableiten lassen. So arbeiten Ortega-Argilés, R. u. a. (2009) z.B. heraus, dass eine gezielte Unterstützung der F\&E-Aktivitäten bestimmter KMU-Untergruppen zur Beseitigung von größenbedingten Benachteiligungen durchaus gerechtfertigt ist. Aufgrund der zentralen Bedeutung der Kundennähe für viele innovationsaktive KMU fordert hingegen Semlinger, K. (2007) eine Ergänzung solcher vorrangig an technologischen Kompetenzen und hieraus resultierenden Finanzierungsengpässen ansetzenden angebotsseitigen Unterstützungsmaßnahmen durch ein nachfrageseitig orientierte Innovationsförderung. Hierunter würde z.B. eine Stärkung der Marketingkompetenzen in KMU fallen oder aber eine grundsätzliche Stimulierung der Innovationsnachfrage in KMU-relevanten Märkten, da diese laut Semlinger oftmals unterentwickelt ist und somit kleinere Unternehmen in der möglichen Entfaltung ihres Innovationspotenzials hemmen kann.

17 Siehe hierzu beispielhaft Cohen, W. (1995); Malerba, F. (2005); von Tunzelmann, N. und Acha, V. (2005).

18 In Anlehnung an Maußner, A. (1994), S. 5, werden im Rahmen der vorliegenden Dissertation unter dem Begriff Konjunktur die wellenförmigen „Schwankungen wirtschaftlicher Aktivität um einen Normalzustand“ verstanden, die sich im idealtypischen Fall eines mehrjährigen Konjunkturzyklus anhand von vier Phasen unterteilen lassen (1. Abschwung bzw. Rezession; 2. Konjunkturtal bzw. Depression; 3. Aufschwung bzw. Erholung; 4. Hochkonjunktur bzw. Boom). In empirischen Analysen zeigen sich Konjunkturschwankungen an den mehr oder weniger zyklisch wiederkehrenden Auf- und Abwärtsbewegungen von Indikatoren wie z.B. dem Bruttoinlandsprodukt, der Beschäftigung oder der Kapazitätsauslastung. Zur möglichen methodischen Operationalisierung von Konjunkturzyklen siehe Kapitel V dieser Dissertation. 
mittelständischen Unternehmen besonders in Zeiten exportinduzierter Wirtschaftskrisen eine Stabilisatorfunktion zugeschrieben wird. Im Rahmen einer KMU-typischen Nischenstrategie würden sich zudem häufig enge und auf Vertrauen basierende Kundenbeziehungen entwickeln, die sich im Konjunkturverlauf ebenfalls stabilisierend auswirken können. Ferner wäre in diesem Zusammenhang auch die hohe Nähe vieler KMU zum privaten Verbrauch von Relevanz, da es sich hierbei um ein relativ wenig schwankungsanfälliges Nachfrageaggregat handelt. Nach Erixon (2009), S. 297, sprechen des Weiteren unter konjunkturellen Gesichtspunkten verschiedene Gründe für eine stabilere Beschäftigungslage in KMU. Denn aufgrund ihrer bereits kleinen Unternehmensgröße wäre für KMU ein Beschäftigungsabbau in Zeiten einer Rezession per se eine weniger in Frage kommende Bewältigungsstrategie als sie es für Großunternehmen ist. Durch die Möglichkeit zum zeitlich flexibleren Einsatz eines Eigentümer-Unternehmers wären viele kleinere Unternehmen darüber hinaus eher dazu in der Lage, auf der betrieblichen Ebene die Auswirkungen von konjunkturellen Schwankungen abzufedern. Zudem würden krisenbedingt notwendig werdende Lohnkürzungen in KMU leichter auf Akzeptanz stoßen, da dort häufiger eigene Familienmitglieder beschäftigt sind. Familienunternehmen wären in einem Konjunkturabschwung darüber hinaus von vornherein weniger geneigt Beschäftigung abzubauen. Alles in allem deutet somit aus theoretischer Sicht einiges darauf hin, dass kleinere Unternehmen weniger sensibel auf die zyklischen Schwankungen der gesamtwirtschaftlichen Konjunkturlage reagieren und sich diesbezüglich folglich stabiler darstellen als größere Unternehmen. ${ }^{19}$

Vorliegende empirische Studien haben primär den Zusammenhang zwischen dem Konjunkturgeschehen und der unternehmensgrößenbezogenen Beschäftigungsentwicklung untersucht. Im Falle des industriellen Sektors in Deutschland zeigen Fendel und Frenkel (1998) mit Hilfe von Jahresdaten für den Zeitraum 1978 bis 1992, dass die Entwicklung der aggregierten Nettobeschäftigung $^{20}$ im Falle von Großunternehmen stärker auf gesamtwirtschaftliche Konjunkturschwankungen reagiert als es in der Gruppe der KMU (hier: zwischen 20 und 500 Beschäftigte) der Fall ist. Dieses Ergebnis erwies sich unter Berücksichtigung verschiedener Kontrollvariablen (Branchenzugehörigkeit, diverse Struktureffekte) als robust. Die Autoren sehen vor diesem Hintergrund ihre theoretische Ausgangshypothese einer konjunkturstabilisierenden Wirkung der KMU-Beschäftigungsentwicklung bestätigt. Aus derselben Perspektive untersuchen Varum und Rocha (2011) den potenziellen Einfluss der Größe eines Unternehmens auf dessen prozentuales Beschäftigungswachstum, wobei sie einen besonderen Fokus auf Anpassungsreaktionen während rezessiver Konjunkturphasen legen. Auf der Grundlage von

19 Eine etwaige Stabilisatorfunktion von KMU kann somit freilich auch bedeuten, dass kleinere Unternehmen in einem Konjunkturaufschwung z.B. weniger stark von einer wachsenden Exportnachfrage profitieren können oder zögerlicher neue Mitarbeiter einstellen als größere Unternehmen. Sowohl Krämer, W. (2003) als auch Erixon, F. (2009) stimmen zudem darin überein, dass KMU aufgrund ihrer tendenziell schwächeren Finanzierungsposition von konjunkturellen Abschwungphasen auch stärker betroffen sein können als Großunternehmen (z.B. aufgrund von Schwierigkeiten bei der ausreichenden Versorgung mit Bankkrediten und einem gleichzeitigen Mangel an alternativen Finanzierungsformen).

20 Der Nettobeschäftigungseffekt ergibt sich in diesem Falle als Saldo der aggregierten Beschäftigungsgewinne und -verluste je Unternehmensgrößenklasse. 
Jahresdaten für den Zeitraum 1988 bis 2007 zeigen ihre Ergebnisse dabei im Falle des portugiesischen Verarbeitenden Gewerbes zunächst, dass das Beschäftigungswachstum zwar sowohl in KMU (hier: bis zu 249 Beschäftigte) als auch in Großunternehmen von konjunkturellen Abschwüngen negativ betroffen ist. Dieser Effekt fällt jedoch im Falle von Großunternehmen stärker aus, was nach Ansicht der Autoren auf eine wichtige Stabilisatorfunktion von KMU deutet. Den Ergebnissen von Varum und Rocha (2011) zufolge wächst die Beschäftigung in Großunternehmen in anschließenden Aufschwungphasen auch wieder schneller an als diejenige von KMU. Folglich finden die Ergebnisse von Fendel und Frenkel (1998) ihre Bestätigung, wonach KMU über die verschiedenen Phasen eines Konjunkturzyklus hinweg eine grundsätzlich geringere Beschäftigungsdynamik aufweisen als Großunternehmen und somit - aus relativer Perspektive - stabilisierend wirken. Die Studie von Davidsson u.a. (1999) deutet in diesem Zusammenhang zudem auf die mögliche Relevanz von qualitativen KMU-Merkmalen. Mit Hilfe von Jahresdaten für alle schwedischen Wirtschaftsbereiche im Zeitraum 1988 bis 1994 können die Autoren zunächst wiederum durch einen absoluten und relativen Vergleich von aggregierten Beschäftigungsgewinnen und -verlusten aufzeigen, dass KMU (hier: bis zu 200 Beschäftigte) während der starken Rezession der schwedischen Volkswirtschaft im Zeitraum 1990 bis 1993 in größerem Ausmaß zur Stabilisierung der gesamtwirtschaftlichen Beschäftigungslage beigetragen haben als Großunternehmen. Eine differenziertere Betrachtung des KMU-Sektors lieferte diesbezüglich weitere Aufschlüsse. Demnach waren hierfür in erster Linie kleine Einbetriebsunternehmen verantwortlich, die häufig durch die Mitarbeit eines Eigentümer-Unternehmers und seiner Familienmitglieder geprägt sind. ${ }^{21}$

Alles in allem sprechen somit in der Tat verschiedene empirische Belege für eine Stabilisatorfunktion von KMU im Rahmen des gesamtwirtschaftlichen Konjunkturverlaufs. Aus unterschiedlichen Gründen ergibt sich vor dem Hintergrund der vorliegenden Literatur dennoch die Relevanz von weiterführenden Forschungsanstrengungen auf diesem Gebiet. Dies trifft zum einen auf die verwendete Datenbasis zu. Wie im vorrangegangenen Absatz deutlich wurde, liegen bisherigen Studien in der Regel jährliche Werte zur Untersuchung der konjunkturellen Muster in der Beschäftigungsentwicklung von KMU und Großunternehmen zu Grunde. Zur Analyse von eher kurzfristigen Konjunkturschwankungen wären jedoch vor allem Monatsbzw. Quartalsdaten von Interesse, da diese - so sie denn verfügbar sind - die Datenbasis um wichtige unterjährige Informationen erweitern würden. Zum anderen deuten bereits die dargestellten Ergebnisse von Davidsson u.a. (1999) auf die Relevanz von differenzierenden Analysen in diesem Themenzusammenhang hin, um die Heterogenität von KMU auch im Falle des Konjunkturgeschehens stärker berücksichtigen zu können. Letzteres wäre auch in zukünftigen Untersuchungen von Bedeutung, die zur weiteren empirischen Fundierung der Stabilisatorhypothese auch die Absatzseite von KMU und Großunternehmen - sprich die konjunkturelle Stabilität der für diese jeweils relevanten Marktnachfrage - in den Fokus ihrer Betrachtung rücken könnten. Eine Orientierung an diesen Untersuchungspunkten könnte auf

21 Siehe auch Moscarini, G. und Postel-Vinay, F. (2009) für eine umfangreiche länderübergreifende Analyse, deren Ergebnisse ebenfalls die These einer im Falle von Großunternehmen höheren Konjunkturanfälligkeit der Beschäftigungsentwicklung stützt. 
diesem Wege ein tiefergehendes Verständnis der gesamtwirtschaftlichen Rolle von KMU (vor allem während wirtschaftlicher Krisenzeiten) erlauben.

\section{3 Überblick zu den Einzelbeiträgen der Dissertation}

Durch den vorangegangenen Literaturüberblick sollte deutlich werden, dass insbesondere die beiden Themenfelder Innovation und Konjunktur jeweils für sich genommen geeignet sind, kleinere Unternehmen auf ihre möglichen Besonderheiten hin zu analysieren. ${ }^{22}$ Die Einzelbeiträge der Dissertation lassen sich in diesen Fachkontext einordnen (vgl. im Folgenden Abbildung 2). Alle vier verfolgen einen empirisch-quantitativen Untersuchungsansatz. In Abhängigkeit vom Untersuchungsziel kommen dabei entweder Analysemethoden zur Überprüfung hergeleiteter Hypothesen (Varianzanalyse, Probitanalyse) oder empirische Verfahren zur Aufdeckung von inhaltlich relevanten Beziehungszusammenhängen in den vorliegenden Daten (Faktorenanalyse, Clusteranalyse, Zeitreihenzerlegung) zur Anwendung. Die Operationalisierung von KMU orientiert sich im Einzelfall an der zu beantwortenden Forschungsfrage und den Gegebenheiten der jeweiligen Datenbasis.

Die ersten drei Einzelbeiträge widmen sich dem Schutz von Innovationen in KMU. Die Fähigkeit zur Hervorbringung von Innovationen stellt zwar eine wesentliche Grundlage für die Wettbewerbsfähigkeit von privatwirtschaftlichen Unternehmen dar. Die Sicherstellung des diesbezüglichen Erfolgs hängt jedoch nicht zuletzt davon ab, ob Unternehmen angesichts der raschen Verbreitung von neuem Wissen auch in der Lage sind, von den Erträgen der eigenen Innovationstätigkeit in ausreichendem Maße zu profitieren. Hiermit ist das sogenannte ,appropriability“-Problem angesprochen, nämlich die Schwierigkeiten denen sich Unternehmen bei der Aneignung von Innovationserträgen gegenübersehen und die Anreizprobleme, die hieraus erwachsen können. ${ }^{23}$ Intellectual Property Rights (IPRs) ${ }^{24}$ - allen voran der Patentschutz - spielen in diesem Zusammenhang eine zentrale Rolle. Gemäß der klassischen ökonomischen Begründung sollen Unternehmen durch die Gewährung von IPRs die Resultate ihrer Innovationsaktivitäten vor der Imitation durch Konkurrenten besser schützen können, wodurch wiederum Anreize zur Generierung von volkswirtschaftlich vorteilhaften Innovationen gesetzt würden und sich ein entsprechendes Marktversagen abbauen ließe. ${ }^{25}$ Im Kern auf die-

22 Die direkten Zusammenhänge zwischen dem Konjunkturverlauf und der Innovationstätigkeit von Unternehmen wurden hierbei ausgeblendet. Einen diesbezüglichen Literaturüberblick liefern z.B. Geroski, P. A. und Walters, C. F. (1995), S. 917ff. Für entsprechende Analysen vor dem Hintergrund der jüngsten Finanz- und Wirtschaftskrise siehe auch Filippetti, A. und Archibugi, D. (2011) und Zimmermann, V. (2010).

23 Vgl. Teece, D. J. (1986); Teece, D. J. (2006); Geroski, P. (1995), S. 91ff.

24 IPRs lassen sich in gewerbliche Schutzrechte (z.B. Patente, Geschmacksmuster, Marken) und den Urheberrechtsschutz unterteilen. Zur genaueren Information siehe WIPO (A) und WIPO (B).

25 Vgl. Granstrand, O. (1999), S. 83ff; Guellec, D. und van Pottelsberghe de la Potterie, B. (2007), S. 46ff. Auch im Falle der Gewährung von IPRs als (zeitlich befristete) immaterielle Monopolrechte ergeben sich der Theorie nach jedoch nur suboptimale Innovationsanreize für rationale Investoren, da diese den Umfang ihrer Innovationsaktivitäten nur an den erwarteten Monopolgewinnen ausrichten und nicht am potenziellen gesellschaftlichen Gesamtnutzen (Vgl. Greenhalgh, C. und Rogers, M. (2007), S. 544f). 
ser Grundannahme beruhend, gehört die Förderung einer intensiveren bzw. effektiveren IPRNutzung durch KMU zu den aktuellen Punkten auf der innovationspolitischen Agenda. ${ }^{26}$ Denn trotz ihrer innovatorischen Bedeutung greifen KMU nur unterdurchschnittlich häufig auf IPRs zurück. In der wirtschaftspolitischen Diskussion besteht angesichts dieser Tatsache die Sorge, dass kleinere Unternehmen aufgrund von größenbedingten Benachteiligungen im IPR-System nicht ausreichend genug auf die staatlich gewährte Aneignungsmöglichkeit zurückgreifen können. Die Beseitigung von hieraus resultierenden Innovationshemmnissen stellt daher ein übergreifendes Ziel der KMU-orientierten Fördermaßnahmen im IPR-Bereich dar.

Abbildung 2: Überblick zu den Einzelbeiträgen der Dissertation

Kleine und mittlere Unternehmen mit Besonderheiten? - Beiträge zur Mittelstandsforschung an den Beispielen von Innovation und Konjunktur

\begin{tabular}{|c|c|c|c|c|}
\hline Themenfeld & & Innovation & & Konjunktur \\
\hline Kapitel & II & III & IV & $\mathrm{V}$ \\
\hline Titel & $\begin{array}{l}\text { Kleinunternehmen } \\
\text { und der Schutz von } \\
\text { Innovationen - } \\
\text { benachteiligt oder } \\
\text { einfach anders? }\end{array}$ & $\begin{array}{l}\text { To protect or not to } \\
\text { protect? Modes of } \\
\text { appropriability in } \\
\text { the small enterprise } \\
\text { sector }\end{array}$ & $\begin{array}{l}\text { Innovation pro- } \\
\text { tection practices in } \\
\text { small and medium } \\
\text { enterprises (SMEs) }\end{array}$ & $\begin{array}{c}\text { Das Handwerk- } \\
\text { ein Konjunktur- } \\
\text { stabilisator? }\end{array}$ \\
\hline Datenbasis & $\begin{array}{c}\text { Community } \\
\text { Innovation Survey } \\
\text { 2000/2001 (CIS 3) }\end{array}$ & $\begin{array}{c}\text { Mannheimer } \\
\text { Innovationspanel } \\
2005\end{array}$ & $\begin{array}{c}\text { KfW- } \\
\text { Mittelstandspanel } \\
2007\end{array}$ & $\begin{array}{c}\text { Handwerks- } \\
\text { berichterstattung } \\
\text { 1996-2009 }\end{array}$ \\
\hline $\begin{array}{l}\text { Unter- } \\
\text { suchungs- } \\
\text { gruppe }\end{array}$ & $\begin{array}{l}\text { Innovative Unter- } \\
\text { nehmen zusammen- } \\
\text { gefasst in drei Grö- } \\
\text { ßenklassen (klein, } \\
\text { mittel, groß) }\end{array}$ & $\begin{array}{c}\text { Innovative } \\
\text { Kleinunternehmen } \\
\text { (hier: } 5 \text { bis } 49 \\
\text { Beschäftigte) }\end{array}$ & $\begin{array}{c}\text { Innovative KMU } \\
\text { (hier: bis zu } 499 \\
\text { Beschäftigte) }\end{array}$ & $\begin{array}{l}\text { Zulassungspflich- } \\
\text { tiges Handwerk } \\
\text { (Anlage A HwO) }\end{array}$ \\
\hline $\begin{array}{l}\text { Analyse- } \\
\text { methode }\end{array}$ & Varianzanalyse & $\begin{array}{c}\text { Faktorenanalyse, } \\
\text { Clusteranalyse }\end{array}$ & Probitanalyse & $\begin{array}{l}\text { Zeitreihen- } \\
\text { zerlegung }\end{array}$ \\
\hline
\end{tabular}

Verschiedene empirische Befunde sprechen in der Tat dafür, dass kleinere Unternehmen aufgrund von größenbedingten Defiziten einen geringeren Nutzen aus dem IPR-System ziehen können als größere Unternehmen. Entsprechende Bereiche, in denen sich KMU potenziellen Benachteiligungen gegenübersehen, sind z.B. die Anmeldungs- und Aufrechterhaltungskosten von Patenten, die im Falle von langwierigen Durchsetzungsstreitigkeiten benötigten finanziel-

26 Vgl. z.B. PRO INNO Europe (2007); Radauer, A. u. a. (2007); Blind, K. u. a. (2009),S. 94ff; BMWi (2010). 
len Ressourcen sowie die Schwierigkeiten bei der Nutzbarmachung der in öffentlich zugänglichen Patentdatenbanken gespeicherten Informationen. ${ }^{27}$ Trotz dieser empirischen Evidenz steht jedoch eine abschließende Antwort auf die Frage weiterhin aus, warum IPRs nur unterdurchschnittlich häufig durch kleinere Unternehmen genutzt werden. Nach Scotchmer (2004), S. 39ff und S. 259ff, kann nicht automatisch vorausgesetzt werden, dass es sich bei IPRs von vornherein um den besten Weg zur Lösung des „,appropriability“-Problems handelt. ${ }^{28}$ Bisher wurde jedoch selten nach der grundsätzlichen Relevanz von IPRs für die Aneignung von Innovationserträgen in KMU gefragt. $^{29}$ Dies ist insofern bedeutsam, da sich Innovationsprozesse in kleineren Unternehmen - wie in Abschnitt 2.1 diskutiert - in gewisser Hinsicht von denjenigen in größeren Unternehmen unterscheiden. Es kann folglich nicht ausgeschlossen werden, dass in KMU die Aneignung von Innovationserträgen ebenfalls charakteristische Besonderheiten aufweist. Ohne die vorliegenden Hinweise auf das Vorhandensein von größenbedingten Benachteiligungen im IPR-System in Frage stellen zu wollen, möchten die Einzelbeiträge in den Kapiteln II bis IV dieser Dissertation daher ein differenzierteres Bild zum Aneignungsverhalten von innovativen KMU und damit letztlich zur Bedeutung von IPRs für kleinere Unternehmen liefern. Sie ordnen sich auf diesem Wege in eine Reihe von Arbeiten aus dem Bereich der Innovationsforschung ein, welche die unternehmens- und branchenspezifische Rolle von verschiedenen formellen und informellen Aneignungsmechanismen (z.B. IPRs, zeitlicher Vorsprung vor Wettbewerbern, Geheimhaltung etc.) behandeln. ${ }^{30}$

Den Rahmen des vierten Einzelbeitrags bildet die Finanz- und Wirtschaftskrise 2008/2009 in Deutschland. Diese führte mit einer Schrumpfung des preisbereinigten Bruttoinlandsprodukts um -4,7\% im Jahr 2009 zur bisher stärksten Rezession der bundesdeutschen Wirtschaftsgeschichte. ${ }^{31}$ Auch KMU zeigten sich von den Auswirkungen der Krise stark betroffen. In der öffentlichen Diskussion zum Krisenverlauf wurde jedoch wiederholt die Meinung vertreten, dass gerade die mittelständischen Unternehmen als wichtige Stabilisatoren der Gesamtwirtschaft gewirkt hätten. Denn im Vergleich zu den vom Einbruch des Außenhandels besonders schwer in Mitleidenschaft gezogenen Großunternehmen blieb die konjunkturelle Lage der KMU noch vergleichsweise stabil. ${ }^{32}$ Deutlich wurde dies z.B. durch starke unternehmensgrößenbezogene Unterschiede in monatlichen Beurteilungen zur aktuellen Geschäftslage, wie sie

27 Vgl. z.B. Kingston, W. (2000); Hall, M. u. a. (2003); Macdonald, S. (2003); Lanjouw, J. O. und Schankerman, M. (2004).

28 Alternative Wege zur Schaffung von Innovationsanreizen sind aus Sicht der Wirtschaftspolitik z.B. direkte staatliche Fördermaßnahmen (etwa in Form finanzieller Hilfen) oder steuerliche Anreize für F\&E. Für eine Diskussion der Vor- und Nachteile verschiedener Lösungsvarianten siehe Geroski, P. (1995), S. $94 \mathrm{ff}$.

29 Als Beispiele für die wenigen vorliegenden Studien in diesem Themenbereich siehe Kitching, J. und Blackburn, R. A. (2003) und Leiponen, A. und Byma, J. (2009).

30 Siehe diesbezüglich z.B. Levin, R. C. u. a. (1987); Harabi, N. (1995); Cohen, W. M. u. a. (2000); Arundel, A. (2001); González-Álvarez, N. und Nieto-Antolín, M. (2007); Amara, N. u. a. (2008).

31 Vgl. Statistisches Bundesamt, Pressemitteilung Nr. 010 vom 12.01.2011, „Deutsche Wirtschaft 2010: Rasanter Aufschwung nach der Krise“, in: http://www.destatis.de (letzter Zugriff: 20.07.2011).

32 Vgl. KfW u. a. (2010), S. $5 f f$. 
in Abbildung 3 durch entsprechende Salden des $K f W$-ifo-Mittelstandsbarometers ${ }^{33}$ zum Ausdruck kommen. Hierbei handelt es sich um einen saison- und mittelwertbereinigten Indikator, weshalb Werte von größer (kleiner) Null auf eine überdurchschnittliche bzw. positive (unterdurchschnittliche bzw. negative) Konjunkturlage schließen lassen. ${ }^{34}$ Demnach erreichte die konjunkturelle Entwicklung von Großunternehmen in der ersten Jahreshälfte 2009 ihren bisherigen Tiefpunkt seit Beginn des Erhebungszeitraums, der nur mit der Lage im Rezessionsjahr 1993 vergleichbar ist. Der Rückgang der Mittelstandskonjunktur fiel demgegenüber deutlich geringer aus und verharrte zudem auf einem Niveau, das noch etwas über den bisherigen Tiefstständen im Zeitraum 2002/2003 lag. Diese Beobachtung spricht in der Tat dafür, dass KMU im Rezessionsjahr 2009 stabilisierend gewirkt haben. Auch aus der Langfristperspektive gesehen stützt Abbildung 3 darüber hinaus die Stabilisatorhypothese, da die Ausschläge der Geschäftslagebeurteilungen über den gesamten Konjunkturverlauf hinweg im Falle von KMU - tendenziell - geringer ausfallen als bei Großunternehmen.

Abbildung 3: KfW-ifo-Mittelstandsbarometer (Aktuelle Geschäftslage in KMU und Großunternehmen; Monatswerte; saison- und mittelwertbereinigte Salden)

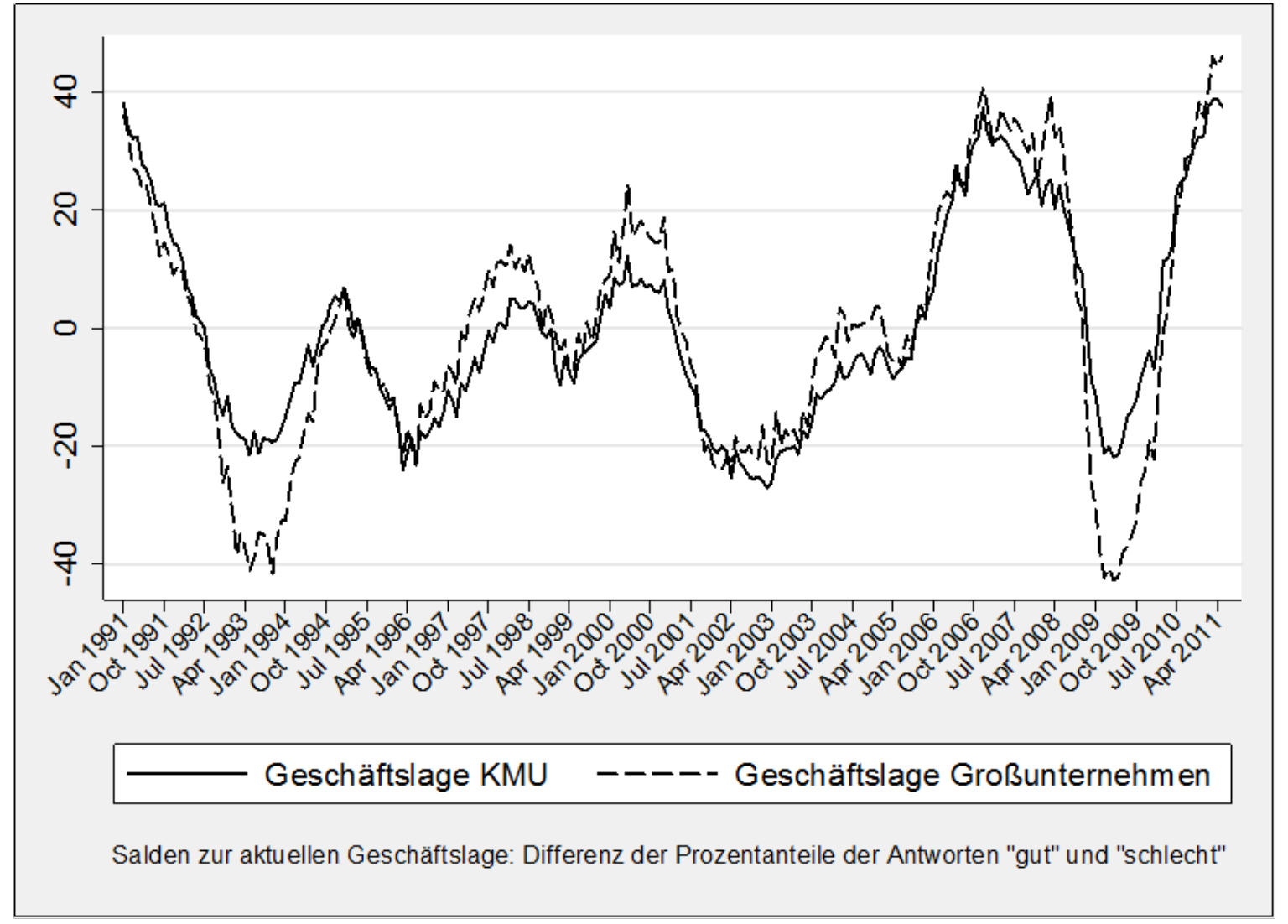

Quelle: KfW Bankengruppe

33 Das KfW-ifo-Mittelstandsbarometer stellt eine Indikatorenfamilie dar, die auf einer größenklassenbezogenen Auswertung des ifo-Geschäftsklimas für die gewerbliche Wirtschaft (Verarbeitendes Gewerbe, Bauhauptgewerbe, Großhandel, Einzelhandel) beruht. Zu den KMU zählen in diesem Falle Unternehmen mit bis zu 500 Beschäftigten und max. 50 Mio. EUR Jahresumsatz mit Ausnahmen im Falle des Einzelhandels (max. 12,5 Mio. EUR Jahresumsatz) und des Bauhauptgewerbes (bis zu 200 Beschäftigte).

Vgl. Borger, K. (2011), S. 3. 
Der vierte Einzelbeitrag möchte vor diesem Hintergrund ein neues Licht auf die etwaige Stabilisatorfunktion von KMU werfen. Konkret erfolgt dies durch eine Untersuchung des Zusammenhangs zwischen dem gesamtwirtschaftlichen Konjunkturgeschehen und der konjunkturellen Lage im KMU-dominierten und durch die Handwerksordnung (HwO) juristisch abgegrenzten Wirtschaftsbereich „Handwerk“.$^{35}$ Denn auch die Handwerkswirtschaft konnte sich der schweren Wirtschaftskrise 2008/2009 nicht entziehen. Nach den vorliegenden Zahlen gingen im Rezessionsjahr 2009 die nominalen Umsätze im zulassungspflichtigen Handwerk gegenüber dem Vorjahr um 5\% zurück. ${ }^{36}$ Prozentual gesehen fiel dieser Rückgang damit sogar stärker aus als der des nominalen Bruttoinlandsprodukts (-3,5 \% gegenüber 2008). Auf der anderen Seite stellte sich jedoch die konjunkturelle Lage vieler Handwerksunternehmen während der Krise im Vergleich zu anderen Wirtschaftsbereichen deutlich stabiler dar. ${ }^{37}$ Das Handwerk hat demnach - anders als die Entwicklung seines Gesamtumsatzes zunächst vermuten lässt - eine konjunkturelle Stabilisatorfunktion innegehabt. Angesichts dieser (scheinbar) gegensätzlichen Sicht auf die Rolle des Handwerks in der zurückliegenden Wirtschaftskrise gewinnt die Frage nach der Konjunkturabhängigkeit des Handwerks mit den hieraus resultierenden Implikationen hinsichtlich der volkswirtschaftlichen Funktion des handwerklich geprägten Teils der Wirtschaft wieder an aktueller Relevanz. In der Forschung wurde der umrissene Sachverhalt in der Vergangenheit bereits vereinzelt aufgegriffen. ${ }^{38}$ Im Kern der Betrachtung stand hierbei letztlich die Überprüfung der Hypothese, wonach das kleinbetrieblich geprägte Handwerk aufgrund seiner höheren Flexibilität und Anpassungsfähigkeit eher zur Stabilisierung der Konjunktur beitragen würde als Großunternehmen. Die vorliegenden empirischen Arbeiten sprechen aber eher gegen eine solche Vermutung, da die Autoren der Handwerkswirtschaft ein überwiegend prozyklisches, d.h. ein konjunkturverstärkendes Verhalten attestieren. Im Hinblick auf die umrissene Lage des Handwerks in der Wirtschaftskrise 2008/2009 untersucht daher der Einzelbeitrag im Kapitel V der Dissertation, ob dieses Ergebnis nicht dennoch einer gewissen Differenzierung bedarf.

\section{Zusammenfassung der Einzelbeiträge: Hintergrund, Ergebnisse, Implikationen}

\subsection{Kleinunternehmen und der Schutz von Innovationen - benachteiligt oder einfach anders?}

Der erste Einzelbeitrag bildet in zweifacher Hinsicht eine Grundlage für die beiden folgenden Untersuchungen zum Schutz von Innovationen in KMU. Zum einen wirft er die im vorangegangenen Abschnitt dargelegte Frage auf, warum kleinere Unternehmen nur in unterdurchschnittlichem Maße von IPRs Gebrauch machen. Vor dem Hintergrund der einschlägigen

\footnotetext{
35 Zur Legaldefinition des deutschen Handwerks und zur vergangenen Entwicklung seiner Unternehmensgrößenstruktur vgl. Dürig, W. u. a. (2004), S. 9ff und S. 41ff.

36 Vgl. Statistisches Bundesamt, Pressemitteilung Nr. 110 vom 19.03.2010, „5,0 \% weniger Umsatz im Handwerk im Jahr 2009“, in: http://www.destatis.de (letzter Zugriff: 21.07.2011).

37 Vgl. ZDH (2009a); ZDH (2009b); IW-Köln (2010).

38 Vgl. Schmidt, K.-H. (1975), S. 1-171; Momm, H.-J. (1983), S. 27ff; Dürig, W. u. a. (2004), S. 66ff.
} 
theoretischen und empirischen Literatur wird ausgeführt, dass diesbezüglich neben einem Nachweis von größenbedingten Benachteiligungen im IPR-System insbesondere vertiefende Analysen zur Aneignung von Innovationserträgen in kleineren Unternehmen von Interesse wären. Zum anderen steckt der erste Einzelbeitrag den empirischen Rahmen für die beiden folgenden Untersuchungen ab, da er auf Grundlage von länderübergreifenden Daten der dritten gemeinschaftlichen Innovationserhebung der Europäischen Union (CIS 3) den grundsätzlichen Zusammenhang zwischen der Größe von innovativen Unternehmen und der Nutzungshäufigkeit von Aneignungsmechanismen untersucht. ${ }^{39}$

Die empirische Analyse führt zu zwei wesentlichen Ergebnissen. Erstens wächst nicht nur die Wahrscheinlichkeit der IPR-Nutzung sondern auch die Nutzungshäufigkeit von informellen Aneignungsmechanismen (hier: zeitlicher Vorsprung, Geheimhaltung, Komplexität der Gestaltung) mit steigender Unternehmensgröße. Es wird jedoch deutlich, dass hierbei stets zwischen absoluten und relativen Effekten zu trennen ist, da aus relativer Perspektive wiederum informelle Aneignungsmechanismen mit sinkender Unternehmensgröße an Bedeutung gewinnen. Dieses Resultat ergänzt damit den Befund von Arundel (2001), der die Notwendigkeit einer relativen Interpretation in diesem Zusammenhang am Beispiel der eingeschätzten Effektivität von Aneignungsmechanismen herausgearbeitet hat. Zweitens sind insbesondere die Ergebnisse zur Größenklasse der Kleinunternehmen auffällig. Der prozentuale Anteil der innovativen Kleinunternehmen, die formelle bzw. informelle Aneignungsmechanismen nutzen, fällt verhältnismäßig gering aus. Vor diesem Hintergrund diskutiert der Beitrag mögliche Besonderheiten im Aneignungsverhalten kleinerer Unternehmen, die neben größenbedingten Benachteiligungen ebenfalls für eine geringere IPR-Nutzung in KMU verantwortlich sein könnten. Die Ergebnisse des ersten Einzelbeitrags deuten damit auf die wirtschaftspolitischen Implikationen von vertiefenden Forschungsanstrengungen in diesem Bereich hin, was zur besseren Veranschaulichung und aufgrund der Relevanz für die beiden folgenden Einzelbeiträge an dieser Stelle detaillierter als in Kapitel II ausgeführt wird.

Eine unterdurchschnittliche IPR-Nutzung durch KMU ist aus wirtschaftspolitischer Sicht letztlich nur dann besorgniserregend, wenn erstens überhaupt ein Marktversagen im Sinne des oben dargelegten „appropriability“-Problems vorliegt und zweitens kleinere Unternehmen im gegebenen Fall anstatt mit IPRs nicht auch mit Hilfe von alternativen Aneignungsmechanismen in ausreichendem Maße von den Erträgen der eigenen Innovationtätigkeit profitieren können. ${ }^{40}$ Vor diesem Hintergrund erscheint der empirische Befund einer mit sinkender Unternehmensgröße wachsenden relativen Bedeutung von informellen Aneigungsmechanismen in einem neuen Licht. Des Weiteren lassen die im ersten Einzelbeitrag diskutierten et-

39 Betrachtet werden aggregierte Ergebnisse für drei Unternehmensgrößenklassen (klein = 10-49 Beschäftigte, mittel = 50 bis 249 Beschäftigte und groß = mehr als 250 Beschäftigte). Hinweis: Zur Vereinheitlichung mit den beiden folgenden Einzelbeiträgen wird an dieser Stelle von formellen und informellen Aneignungsmechanismen gesprochen. Im bereits veröffentlichten ersten Einzelbeitrag (siehe Kapitel II) wird eine hiervon leicht abweichende Terminologie verwendet, nämlich: formelle und strategische Aneignungsmechanismen.

40 Vgl. Dosi, G. u. a. (2006), S. 1111ff und S. 1116f; Jensen, P. H. und Webster, E. (2006), S. $45 f$ und S. 54. 
waigen Besonderheiten im Aneignungsverhalten kleinerer Unternehmen darauf schließen, dass sich die der klassischen ökonomischen IPR-Begründung zu Grunde liegende Problematik der Nicht-Aneigbarkeit neuen Wissens womöglich gerade in innovativen KMU weniger stellt als in Großunternehmen. Denn die Existenz eines Marktversagens ist im vorliegenden Fall vor allem von der Geschwindigkeit abhängig, mit der eine innovative Wissensbasis von potenziellen Imitatoren in ihren Wesensmerkmalen nachvollzogen werden kann. Nach Reed und DeFillippi (1990), S. 91ff, beeinflussen von einem theoretischen Standpunkt aus im Kern drei Eigenschaften von Wissen die Möglichkeit zur Imitation: Kodifizierbarkeit, Komplexität und Spezifität. Alle drei Wissensdimensionen können jeweils Quelle einer „natürlichen“ Imitationsbarriere sein, was - insbesondere im Falle ihrer Interaktion - die Aussicht auf eine erfolgreiche Aneignung von Innovationserträgen von sich aus verbessert.

Kodifizierbarkeit bezieht sich auf den Umstand, dass Wissen sowohl expliziter als auch impliziter Natur sein kann. Explizite bzw. leicht kodifizierbare Wissensbestandteile sind als beschreibbare Informationen zu verstehen, wodurch ein (unerwünschter) Wissenstransfer relativ leicht möglich ist. Implizites Wissen („tacit knowledge“) kommt hingegen in mehr oder weniger unbewusst erworbenen Fertigkeiten zum Ausdruck. Es kann daher kaum oder gar nicht formalisiert und beschrieben sowie oftmals nur unter erheblichem Aufwand transferiert werden (so z.B. durch das gezielte Abwerben des Wissensträgers). Umso impliziter sich die innovative Wissensbasis eines Unternehmens folglich darstellt, umso schwieriger gestaltet sich auch eine unerwünschte Imitation. Komplexität basiert wiederum auf der Kompetenz, zur Bereitstellung eines Innovationsbeitrags verschiedene Fähigkeiten und Ressourcen auf eine bestimmte bzw. einzigartige Art und Weise miteinander zu kombinieren. Mit wachsendem Komplexitätsgrad neuen Wissens wird eine erfolgreiche Imitation weniger wahrscheinlich. Spezifität bezieht sich hingegen auf die Kontextabhängigkeit von Wissen. Sie resultiert aus dem gezielten Einsatz von Fähigkeiten und Ressourcen zur Befriedigung von individuellen Kundenwünschen. Kerngedanke dieser Wissensdimension ist, dass innovierende Unternehmen mit ihren Kunden eine Art Symbiose eingehen, was sich in einer beiderseitig vorteilhaften und langandauernden Geschäftsbeziehung äußern kann. Durch eine solche individuelle Spezialisierung von Kompetenzen steigen die Alleinstellungsmerkmale der zugrundeliegenden Wissensbasis, wodurch eine Imitation durch die Konkurrenz weniger wahrscheinlich wird und sich entsprechende Wettbewerbsvorteile ergeben können. ${ }^{41}$

Zumindest ein Teil der im ersten Einzelbeitrag diskutierten potenziellen Besonderheiten im Aneignungsverhalten von kleineren Unternehmen lässt sich in diesen ressourcenökonomischen Erklärungsrahmen einordnen. Zum einen ist gerade die operative Wissensbasis in kleineren Unternehmen eher impliziter Natur, da Innovationsaktivitäten dort häufig in den Routinen des Tagesgeschäfts eingebettet sind und stark erfahrungsbasiert vonstattengehen. ${ }^{42}$ Hinsichtlich der Relevanz von IPRs für den Schutz von Innovationen in KMU ist dies mit zwei möglichen

41 Vgl. Reed, R. und DeFillippi, R. J. (1990), S. 91ff; Zander, U. und Kogut, B. (1995), S. 79ff; Teece, D. J. (2003), S. 133ff.

42 Vgl. Nooteboom, B. (1994), S. 336f; Rantakyrö, L. (2005), S. $220 f f$. 
Implikationen verbunden. Erstens dürften z.B. Patente tendenziell weniger in Frage kommen, da eine Patentanmeldung die Reduktion der zu schützenden Wissensbestandteile auf beschreibbare, d.h. kodifizierbare Informationen erfordert. Gestützt wird diese Vermutung durch empirische Befunde, wonach mit einer höheren Kodifizierbarkeit der Wissensbasis eines Unternehmens auch dessen Neigung zur Nutzung von Patenten als Aneignungsmechanismus wächst. ${ }^{43}$ Zweitens dürfte der Patentschutz gleichzeitig weniger notwendig für kleinere Unternehmen sein, weil diese aufgrund der imitationshemmenden Wirkung von implizitem Wissen den potenziellen Vorteil haben, sich die Erträge ihrer Innovationstätigkeit auch auf diesem Wege erfolgreich anzueignen. ${ }^{44}$

Zum anderen leisten gerade innovative Kleinunternehmen ihre Innovationsbeiträge häufig auf Grundlage einer ausgeprägten Interaktion von komplexem und spezifischem Wissen. In der einschlägigen Literatur kommt dies dadurch zum Ausdruck, dass insbesondere im Vorhandensein einer unternehmensspezifischen Problemlösungskompetenz eine Chance kleinerer Unternehmen für eine erfolgreiche Innovationstätigkeit gesehen wird. ${ }^{45}$ Wesentliche, sich gegenseitig stark bedingende Bestandteile einer solchen Abgrenzungsstrategie sind eine ausgeprägte Spezialisierung, die z.B. durch eine Konzentration auf Marktnischen zum Ausdruck kommt, ferner die Kompetenz verschiedene Fähigkeiten und Ressourcen auf eine bestimmte bzw. einzigartige Art und Weise miteinander zu kombinieren und überdies eine hohe Kundennähe, welche sich in neuen Problemlösungen für einen sehr beschränkten Anwenderkreis niederschlagen kann. Die auf Grundlage einer unternehmensspezifischen Problemlösungskompetenz erworbenen Alleinstellungsmerkmale dürften daher aus Sicht von kleineren Unternehmen die Notwendigkeit verringern, IPRs zum Schutz von Innovationen zu nutzen. ${ }^{46}$

\subsection{To protect or not to protect? Modes of appropriability in the small enterprise sector}

Der zweite Einzelbeitrag beschränkt sich in seiner Analyse von unterschiedlichen Aneignungsstrategien auf eine Betrachtung des Kleinunternehmenssektors (hier: innovative Unternehmen mit 5 bis 49 Beschäftigten), weil vor allem von diesem KMU-Bereich allein aufgrund der besonders hohen Zahl an zugehörigen Unternehmen eine große Heterogenität im Aneignungsverhalten erwartet werden kann. Zudem soll hierdurch auch ein Beitrag zur stärkeren Akzentuierung der charakteristischen Besonderheiten von innovativen Kleinunterneh-

43 Vgl. Nieto, M. und Pérez-Cano, C. (2004); González-Álvarez, N. und Nieto-Antolín, M. (2007). Die Möglichkeit zum Rückgriff auf den Patentschutz dürfte freilich auch deshalb geringer sein, weil der inkrementelle Neuheitsgrad vieler KMU-Innovationsbeiträge häufig eine Patentanmeldung nicht zulässt.

44 Vgl. Nooteboom, B. (1994), S. 334ff; Hurmelinna-Laukkanen, P. und Puumalainen, K. (2007), S. 96f.

45 Vgl. z.B. Lahner, J. (2004), S. 285; Nooteboom, B. (1994), S. 334f; Mazzarol, T. und Reboud, S. (2009), S. 214; Frank, H. u. a. (2010), S. $24 \mathrm{f}$.

46 Hierauf deuten bereits die Interviewbefragungen von Kitching, J. und Blackburn, R. A. (2003), S. 23ff, und Lahner, J. (2004), S. 260f und S. 312ff. Deren Ergebnisse lassen darauf schließen, dass kleinere Unternehmen neben den Kosten der Anmeldung und Durchsetzung womöglich auch deshalb auf einen ressourcenzehrenden Patentschutz verzichten, weil sie diesen aufgrund einer Konzentration auf kleine Nischenmärkte bzw. der Überzeugung vom Alleinstellungcharakter eigener Kompetenzen nicht als notwendig erachten. 
men gerade abseits des Hightech-Bereichs geliefert werden, welche in jüngster Zeit verstärkt angemahnt worden ist. ${ }^{47}$ Im vorliegenden Fall zeigt eine entsprechend orientierte Auswertung von Daten des Mannheimer Innovationspanels, dass sich der Kleinunternehmenssektor unter aneignungsstrategischen Gesichtspunkten in vier Segmente unterteilen lässt. Diese in sich homogenen Unternehmensgruppen werden daraufhin anhand verschiedener Merkmale (z.B. Relevanz von F\&E-Aktivitäten, Art und Neuheitsgrad der getätigten Innovationen, Auswirkungen von Innovationsaktivitäten auf den Unternehmenserfolg, Bedingungen des Wettbewerbsumfelds etc.) charakterisiert. Durch eine solche Einordnung in den unternehmensund branchenspezifischen Kontext soll ein besseres Verständnis bezüglich der verschiedenen von innovativen Kleinunternehmen verfolgten Aneignungsstrategien erlangt werden. ${ }^{48}$

Den Untersuchungsergebnissen zufolge wird Patenten und anderen IPRs von einem relativ kleinen Teil des Kleinunternehmenssektors durchaus eine hohe Bedeutung für den Schutz von Innovationen eingeräumt. Für die überwiegende Mehrzahl der innovativen Kleinunternehmen besteht die Kernfrage jedoch offenbar nicht darin, IPRs zu nutzen oder nicht, sondern ob überhaupt eine Notwendigkeit zur Ergreifung von aktiven Schutzmaßnahmen besteht. ${ }^{49}$ Des Weiteren wird deutlich, dass sich die Nutzung von formellen und informellen Aneignungsmechanismen keineswegs gegenseitig ausschließen muss. Vielmehr basiert die Effektivität einzelner Aneignungsstrategien in vielen Fällen offenbar nicht zuletzt auf der Kombination von unterschiedlichen aber sich gegenseitig ergänzenden Schutzmaßnahmen. Beispielsweise wird eine Geheimhaltungsstrategie und ein zeitlicher Vorsprung vor der Konkurrenz sowohl von Kleinunternehmen, die gleichzeitig auch auf IPRs zum Schutz von Innovationen setzen, als auch von einem Teil des Kleinunternehmenssektors, in dem die Komplexität der technischen Produktgestaltung als informeller Aneignungsmechanismus von Relevanz ist, für wichtig erachtet. Demgegenüber werden technische Schutzrechte wie Patente und eine komplexe Produktgestaltung im Rahmen einer Aneignungsstrategie kaum miteinander kombiniert. Hierbei handelt es sich folglich um alternative Schutzmaßnahmen. ${ }^{50}$

Daneben liefert die eingehende Charakterisierung der vier Unternehmensgruppen vertiefte Erkenntnisse bezüglich der Wirksamkeit und der Verfügbarkeit sowohl von IPR-orientierten als auch von nicht-IPR-orientierten Aneignungsstrategien. Hierdurch zeigt sich z.B., dass der patentorientierte Teil des Kleinunternehmenssektors (11\% der Untersuchungsstichprobe) weni-

47 Vgl. Abel, R. (2006); Lahner, J. (2008); Frank, H. u. a. (2010).

48 Sowohl inhaltlich als auch methodisch knüpft der erste Einzelbeitrag dabei an eine Reihe von vorliegenden Studien an, die sich zur möglichen Erfassung der Heterogenität im Innovationsverhalten der Abgrenzung und Klassifizierung von unterschiedlichen „Innovationstypen“ (Taxonomies of innovation) widmen. Vgl. hierzu z.B. Pavitt, K. (1984); Malerba, F. und Orsenigo, L. (1997); Evangelista, R. (2000); Hollenstein, H. (2003); Jong de, J. und Marsili, O. (2006); Peneder, M. (2010).

49 Somit erklärt sich auch der im Rahmen des ersten Einzelbeitrags aufgezeigte Umstand, dass die absolute Nutzungshäufigkeit sowohl von formellen als auch von informellen Aneignungsmechanismen mit steigender Unternehmensgröße wächst. Siehe Abschnitt 3.1. in diesem Kapitel oder Kapitel II.

50 Dies bestätigt die Analyseergebnisse von Amara, N. u. a. (2008), wonach der Patentschutz und die Komplexität der Gestaltung aus Unternehmenssicht unterschiedliche Aneignungsalternativen darstellen. 
ger durch „KMU-typische“ Innovationsmerkmale (etwa diskontinuierliche F\&E-Aktivitäten, hoher Stellenwert der Kundennähe, Relevanz inkrementeller Qualitätsverbesserungen etc.) geprägt ist als die überwiegende Mehrzahl der befragten innovativen Kleinunternehmen. Die diesbezüglichen Ausführungen im Rahmen des zweiten Einzelbeitrags lassen daher darauf schließen, dass neben größenbedingten Benachteiligungen im IPR-System auch der spezifische Charakter der Innovationsaktivitäten in kleineren Unternehmen für die unterdurchschnittliche IPR-Nutzung in KMU verantwortlich sein dürften. Besonders gut deutlich wird dies anhand der hohen Bedeutung des Aneignungsmechanismus „Komplexität der Gestaltung“ für eine Reihe von kleinunternehmerischen Innovatoren. Wie am Beispiel des oben dargestellten ressourcenökonomischen Erklärungsansatzes ausgeführt wurde, kann eine technisch komplexe Produktgestaltung insbesondere im Falle einer gleichzeitigen Spezialisierung auf individuelle Kundenwünsche eine effektive Schutzwirkung entfalten. Die Untersuchungsergebnisse sprechen eben hierfür, da innovative Kleinunternehmen, welche auf diesen Aneignungsmechanismus zurückgreifen, gleichzeitig eine hohe Flexibilität und Servicequalität bei Kundenwünschen als bedeutenden Wettbewerbsfaktor erachten. Des Weiteren stützt die empirische Auswertung die Einschätzung von Mazzarol und Reboud (2009), S. 214, wonach in diesem Zusammenhang der inkrementelle Charakter vieler kleinunternehmerischer Innovationen eine wichtige Rolle spielt. Denn im Falle von graduellen Anpassungen und Weiterentwicklungen kommt ein Rückgriff auf den Patentschutz aufgrund des vergleichsweise geringen Neuheitsgrades ohnehin weniger in Frage. Damit inkrementelle Innovationen dennoch die Wettbewerbsfähigkeit von kleineren Unternehmen unterstützen können, muss den Autoren zufolge der Komplexitätsgrad von entsprechenden Innovationsbeiträgen hoch genug sein, um die Gefahr einer Imitation durch die Konkurrenz wirksam zu verringern.

Der zweite Einzelbeitrag ist mit verschiedenen wirtschaftspolitischen Implikationen verbunden. Im Besonderen gilt dies hinsichtlich der deutlich gewordenen Heterogenität im Aneignungsverhalten von kleineren Unternehmen, da dieser Umstand auf die Relevanz eines entsprechend differenzierten Förderansatzes deutet. Wird daher die grundsätzliche aneignungsstrategische Relevanz von IPRs als Kriterium herangezogen, ist die unterdurchschnittliche IPR-Nutzung durch KMU aus wirtschaftspolitischer Sicht nicht automatisch ein Grund zur Sorge. Denn im Falle von innovativen Kleinunternehmen lässt sich die häufig anzutreffende Wahl von nicht-IPR-orientierten Aneignungsstrategien auch vor dem Hintergrund ihrer internen und externen Kontextbedingungen erklären. Dieser Umstand spricht dabei keineswegs gegen KMU-orientierte Unterstützungsmaßnahmen im IPR-Bereich. ${ }^{51}$ Politische Entschei-

51 Der Studie von Radauer, A. u. a. (2007), S. 23ff, zufolge betrachten (IPR-aktive) KMU die mit einer Nutzung des IPR-Systems verbundenen Transaktionskosten (Kosten der Anmeldung und Durchsetzung eines Schutzrechts bzw. die zeitliche Dauer bis zu dessen Erteilung) als häufigstes Hindernis für eine intensivere Nutzung formeller Schutzrechte. Es kann somit nicht ausgeschlossen werden, dass kleinere Unternehmen formelle Schutzmaßnahmen aus Kostengründen bewusst durch informelle Instrumente substituieren (müssen). Allerdings ist nach der von Radauer, A. u. a. (2007), S. 75ff, vorgenommenen Bewertung von verschiedenen europäischen KMU-Fördermaßnahmen im IPR-Bereich die finanzielle Unterstützung einer Patentanmeldung kaum mit Substitutionseffekten zwischen formellen und informellen Schutzmaßnahmen verbunden. Dieser Umstand dürfte sich unter anderem vor dem Hintergrund der im zweiten Einzelbeitrag aufgezeigten Komplementaritäten zwischen formellen und informellen Aneignungsmechanismen erklären. 
dungsträger sollten sich jedoch bewusst sein, dass sich hiermit in erster Linie nur die Aneignungsbedingungen von bestimmten KMU-Gruppen verbessern lassen dürften. Nichtsdestotrotz zeigen sich vor allem kleinere Unternehmen hinsichtlich der sich ihnen möglicherweise durch das IPR-System bietenden Vorteile von vornherein häufig wenig informiert bzw. sensibilisiert. ${ }^{52}$ Daher erscheinen wirtschaftspolitische Unterstützungsmaßnahmen gerechtfertigt, die KMU in eine bessere Lage versetzen, ihre Entscheidung für oder gegen IPRs auf einer fundierten Informationsgrundlage treffen zu können. Innovationsorientierte KMU, welche z.B. trotz patentierbarer Erfindungen auf die technische Komplexität der Produktgestaltung als Aneignungsmechanismus setzen, würde in der Folge möglicherweise stärker bewusst sein, dass sich im Falle eines Scheiterns ihrer informellen Schutzanstrengungen weniger Möglichkeiten bieten, gegen eine erfolgreiche Imitation rechtlich vorzugehen. Des Weiteren ist angesichts der durch den zweiten Einzelbeitrag deutlich gewordenen Komplementaritäten zwischen formellen und informellen Aneignungsmechanismen der Einschätzung von Blind u.a. (2009), S. 132 und S. 140f, zuzustimmen, wonach in der KMU-orientierten Förderlandschaft anstelle einer einseitigen Fokussierung auf die Höhe der Patentanmeldezahlen zukünftig der grundsätzliche „IP-Managementgedanke“ stärker im Vordergrund stehen sollte. Das würde sich in der Praxis etwa durch eine explizite Einbeziehung von informellen Schutzmaßnahmen in die Beratungsleistung äußern.

Beachtung verdient unter wirtschaftspolitischen Gesichtspunkten zudem der Umstand, dass nach den vorliegenden Untersuchungsergebnissen IPRs auch deshalb keine Rolle für die überwiegende Mehrzahl der innovativen Kleinunternehmen spielen, weil dort offenbar vor allem aufgrund einer kleineren Wahrscheinlichkeit zur Einführung von Produktinnovationen ein aktiver Schutz vor Imitation grundsätzlich von geringer Bedeutung ist. Unterstützungsmaßnahmen zur Verbesserung der Aneignungsbedingungen von KMU sollten daher auch direkt am Innovationspotenzial von kleineren Unternehmen - beispielsweise durch eine kleinunternehmensgerechte Ausgestaltung von Förderprogrammen ${ }^{53}$ oder durch einen stärkeren Fokus auf die hohe Bedeutung der Kundennähe für das Innovationsgeschehen in $\mathrm{KMU}^{54}$ - ansetzen. Eine hierdurch mögliche Intensivierung der Innovationsaktivitäten von kleineren Unternehmen dürfte dann mehr oder weniger „zwangsläufig“ eine stärkere Nutzung von formellen und informellen Schutzmaßnahmen in KMU zur Folge haben.

52 Vgl. WIPO (2003), S. 10f; Greenhalgh, C. und Rogers, M. (2007), S. 563f; Radauer, A. u. a. (2007), S. 26f.

53 Für diesbezügliche Kriterien siehe im Detail Lahner, J. (2008), S. 60f.

54 Die Ergebnisse von Mazzarol, T. und Reboud, S. (2005) zeigen, dass der kommerzielle Innovationserfolg von KMU und damit letztlich deren Möglichkeiten zur Aneignung von Innovationserträgen stark von der Kundenakzeptanz hinsichtlich neuer oder merklich verbesserter Produkte abhängt. Die hohe Bedeutung der Kundennähe kann daher nach Mazzarol, T. und Reboud, S. (2005), S. 490ff, für kleinere Unternehmen auch den Nachteil einer zu starken Abhängigkeit von einzelnen Kunden mit sich bringen, wenn diese keine Bereitschaft zur Würdigung bzw. zur Annahme von entsprechenden Innovationsbeiträgen erkennen lassen. Nach Ansicht der Autoren sollte sich eine Wirtschaftspolitik zur Stärkung des Innovationspotenzials von kleineren Unternehmen deshalb auf die Schnittstelle zwischen KMU und deren Kunden konzentrieren. Zur Relevanz einer solchen nachfrageseitigen Ergänzung der KMU-orientierten Innovationsförderung siehe auch Fußnote Nr. 16. 


\subsection{Innovation protection practices in small and medium enterprises (SMEs)}

Der dritte Einzelbeitrag knüpft an die vorhergehende Untersuchung an, indem er einerseits die Erklärungsfaktoren für die Relevanz von einzelnen Aneignungsmechanismen detaillierter untersucht und andererseits die Betrachtung auf alle innovativen KMU (hier: innovative Unternehmen mit bis zu 499 Beschäftigten) erweitert. Neben der Berücksichtigung von IPRs wird auf Grundlage von Daten des KfW-Mittelstandspanels ein besonders Gewicht auf informelle Schutzmaßnahmen (hier: zeitlicher Vorsprung, Geheimhaltung, Komplexität der Gestaltung, langfristige Bindung qualifizierten Personals) gelegt. Dies geschieht, um der Heterogenität im Aneignungsverhalten von kleineren Unternehmen gerecht zu werden. Der Erkenntnisgewinn des dritten Einzelbeitrags besteht unter anderem darin, die Nutzung von Aneignungsmechanismen aus einer wissensbasierten Perspektive zu untersuchen. Dies erlaubt einen neuen Blick auf den Stellenwert von bestimmten Schutzmaßnahmen in innovativen KMU, was insbesondere im Falle der langfristigen Bindung qualifizierten Personals gilt. Hierbei handelt es sich um einen mitarbeiterorientierten Aneignungsmechanismus, der vor allem für KMU von Bedeutung sein dürfte. Denn Innovationsaktivitäten in kleineren Unternehmen sind - wie oben bereits dargelegt - aufgrund eines hohen Stellenwerts von interaktiven Lernprozessen mit Kunden und Lieferanten sowie der wichtigen Rolle von erfahrungsbasiertem Know-how in der Praxis stark durch die Herstellung und Nutzung von schwer-kodifizierbarem, personengebundenen Wissen („tacit knowledge“) geprägt. ${ }^{55}$

Die empirische Überprüfung einer Reihe von Untersuchungshypothesen zeigt, dass sich die von innovativen KMU vorgenommene Einschätzung hinsichtlich der unternehmensinternen Relevanz von bestimmten Aneignungsmechanismen durch verschiedene Faktoren erklären lässt. Beispielhaft lassen sich an dieser Stelle folgende Ergebnisse des dritten Einzelbeitrags mit ihren wirtschaftspolitischen Implikationen zusammenfassen. Eigene F\&E-Anstrengungen steigern sowohl die Bedeutung von IPRs als auch von informellen Schutzmaßnahmen. Da entsprechende Aktivitäten primär dem Zweck der Generierung von neuem Wissen dienen und angesichts der im vorangegangenen Beitrag aufgezeigten Tatsache, nach der sich die Nutzung von formellen und informellen Aneignungsmechanismen gegenseitig wirksam ergänzen kann, ist dieser Umstand nicht verwunderlich. Die in Abschnitt 2.1 diskutierte Heterogenität im Innovationsprozess von kleineren Unternehmen bezüglich der Rolle von F\&E zeigt sich jedoch daran, dass die langfristige Bindung qualifizierten Personals offenbar auch für nicht-F\&Eaktive Innovatoren (mit einem Stichprobenanteil von über 60\% die Mehrzahl der befragten

55 Hierauf deuten bereits die Ergebnisse der Studie von Kitching, J. und Blackburn, R. A. (2003), S. 19f. Die befragten KMU-Eigentümer-Unternehmer sahen den mit einem möglichen Abgang von Schlüsselpersonal verbundenen Know-how-Verlust als größte Bedrohung für ihr zu schützendes Wissen an und damit z.B. kritischer im Vergleich zu entsprechenden Gefahren durch Konkurrenten oder Kooperationspartner. Auf der Grundlage von acht Fallstudien kommen zudem Olander, H. u. a. (2009), S. 365, zu dem Schluss, dass eine adäquate Personalpolitik gerade für KMU aufgrund der starken Gebundenheit ihrer Wissensbasis an einzelne Personen ein zentraler Aneignungsmechanismus ist. Zudem wäre dies auch deshalb der Fall, weil sie allein aufgrund ihrer kleineren Unternehmensgröße einen Verlust von Schlüsselpersonal weniger gut verkraften können als Großunternehmen. 
innovativen KMU) als effektiver Aneignungsmechanismus wirken kann. ${ }^{56}$ Das Aneignungsverhalten von kleineren Unternehmen ist in diesem Zusammenhang also möglicherweise mit charakteristischen Besonderheiten verbunden. Hierauf deuten noch weitere Ergebnisse des dritten Einzelbeitrags. Diesbezüglich zu erwähnen ist z.B. die empirische Bestätigung der Hypothese, wonach sich handwerkliches Wissen aufgrund seines stark impliziten Charakters in erster Linie durch die langfristige Bindung qualifizierten Personals vor unerwünschter Imitation schützen lässt. ${ }^{57}$ Wirtschaftspolitische Maßnahmen zur Verbesserung der Aneignungsbedingungen von innovativen KMU sollten vor diesem Hintergrund gerade auch auf der Personalseite von kleineren Unternehmen ansetzen. Dies könnte durch eine Unterstützung bei der erfolgreichen Etablierung einer ergebnis- und bindungsorientierten Führungskultur und der gleichzeitigen Umsetzung einer bindungsorientierten Personalpolitik geschehen (etwa durch die Verbreitung von besonders effektiven Managementinstrumenten anhand von „Best Practice“-Beispielen oder der Verdeutlichung ihres konkreten Beitrags zum wirtschaftlichen Unternehmenserfolg). ${ }^{58}$ Aus einer übergreifenden Perspektive wird am Beispiel der diskutierten Rolle einer mitarbeiterorientierten Aneignungsstrategie für innovative KMU zudem noch einmal Folgendes deutlich: Eine einseitige Orientierung an Innovationsindikatoren wie die Höhe der F\&E-Ausgaben oder die Zahl der Patentanmeldungen kann insbesondere das Innovationsgeschehen in kleineren Unternehmen mit seiner häufig auf interaktivem Lernen und erfahrungsbasiertem Know-how liegenden Betonung nur ungenügend abbilden. ${ }^{59}$

Daneben liefern die Ergebnisse des dritten Einzelbeitrags auch Hinweise zur Nutzungsrelevanz von anderen Aneignungsmechanismen. Unmittelbar einleuchtend ist etwa die Beobach-

56 Dies steht im Einklang zu den Ergebnissen von Rammer, C. u. a. (2009). KMU können demzufolge auch ohne eigene F\&E-Anstrengungen einen vergleichbaren Innovationserfolg erzielen wie F\&E-aktive Unternehmen, wenn sie dies durch ein mitarbeiterorientiertes Innovationsmanagement kompensieren und im Idealfall noch durch eine Nutzung von externen Informationsquellen sowie innovationsbezogene Kooperationsaktivitäten ergänzen. Unter einem mitarbeiterorientierten Innovationsmanagement verstehen Rammer, C. u. a. (2009), S. 46f, sowohl die Nutzung einer Reihe von Instrumenten des Personalmanagements (z.B. Zielvereinbarungen mit Führungskräften, Maßnahmen zur Identifikation, Förderung und Bindung von Schlüsselpersonal, Schaffung von Anreizen hinsichtlich des innerbetrieblichen Vorschlagswesens etc.) als auch die Anregung von unternehmensinternem Teamwork (z.B. Förderung eines regelmäßigen informellen Austauschs zwischen Mitarbeitern, gemeinsame Entwicklung von Innovationsstrategien, gegenseitige Unterstützung bei der Lösung von innovationsbezogenen Problemen etc.).

57 Bereits in der Grundlagenarbeit von Polanyi, M. (1958), S. 53 und S. 206, wird ein Ausbildungsverhältnis zwischen Meister und Lehrling, in der Letzterer unter Anleitung und mittels „Learning by Doing“ bestimmte Fertigkeiten entwickelt, als idealtypischer Weg für den Aufbau von implizitem Erfahrungswissen beschrieben. Bezogen auf das Handwerk, in dem diese Form des Wissenserwerbs traditionell stark verankert ist, definieren daher verschiedene Autoren handwerkliches Wissen im Kern als „tacit knowledge“. Vgl. hierzu z.B. Sennett, R. (2008), S. 50ff und S. 94; Gamble, J. (2001), S. 185f; Gamble, J. (2004), S. $190 \mathrm{ff}$.

58 Für Elemente einer ergebnis- und bindungsorientierten Führungskultur und mögliche Ansatzpunkte einer bindungsorientierten Personalpolitik sowie ihre jeweilige Relevanz für innovative Unternehmen siehe Flüter-Hoffmann, C. und Stettes, O. (2011), S. $10 \mathrm{ff}$.

59 Aus einer KMU-Perspektive stützt der dritte Einzelbeitrag damit die Einschätzung von Jensen, M. B. u. a. (2007), S. 690, wonach zur besseren Unterstützung einer innovationsorientierten Wirtschaftspolitik insbesondere bei Indikatoren zur Verdeutlichung von informellen und erfahrungsbasierten Lernprozessen ein Weiterentwicklungsbedarf besteht. Siehe hierzu ebenfalls Lundvall, B.-Å. und Borrás, S. (2005), S. 617. 
tung, dass der Patentschutz in erster Linie für F\&E-intensivere KMU ein entscheidender Aneignungsmechanismus ist. Kleinere Unternehmen setzen jedoch unabhängig davon auch dann auf Patente und andere IPRs zum Schutz von Innovationen, wenn sie im direkten Wettbewerb mit deutlich größeren Konkurrenten stehen. Um zu verhindern, dass Großunternehmen ihren Ressourcenvorteil im Umgang mit dem IPR-System als Wettbewerbsvorteil gegen sie ausspielen, müssen sich solche KMU offenbar bewusst mit Fragen hinsichtlich des rechtlichen Schutzes von geistigem Eigentum auseinandersetzen und auch selbst intensiver auf entsprechende Aneignungsmechanismen zurückgreifen. ${ }^{60}$ Dieser Umstand verdient aus wirtschaftspolitischer Sicht besonderes Augenmerk. Denn es liegen empirische Hinweise vor, wonach KMU im Falle von Schutzrechtsstreitigkeiten mit größeren Unternehmen (etwa im Rahmen von Patentverletzungsverfahren) deutlich benachteiligt sind. ${ }^{61}$ Bezüglich anderer informeller Aneignungsmechanismen zeigt sich beispielsweise Folgendes: Wenn Kooperationsbeziehungen mit anderen Unternehmen oder mit wissenschaftlichen Einrichtungen bestehen, ist ein zeitlicher Vorsprung vor der Konkurrenz für innovative KMU eine eher zu realisierende Schutzmaßnahme. Dies ist insofern relevant, da kleinere Unternehmen vorrangig deshalb mit externen Partnern kooperieren, um die größenbedingten Nachteile ihrer geringeren internen Ressourcenbasis zu kompensieren. ${ }^{62}$ Auch unter aneignungsstrategischen Gesichtspunkten rechtfertigen sich folglich wirtschaftspolitische Maßnahmen, welche die Wettbewerbsfähigkeit von KMU durch eine Förderung von Kooperationsaktivitäten unterstützen sollen.

\subsection{Das Handwerk - ein Konjunkturstabilisator?}

Der vierte Einzelbeitrag vertieft bzw. ergänzt die vorliegenden Forschungsarbeiten zur etwaigen konjunkturellen Stabilisatorfunktion der Handwerkswirtschaft aus verschiedenen Gründen. ${ }^{63}$ Erstens fällt auf, dass in bisherigen Untersuchungen stets das Gesamthandwerk als Untersuchungseinheit gewählt wurde. Gerade der durch die Handwerksordnung juristisch abgegrenzte Wirtschaftsbereich „Handwerk“ ist jedoch keine homogene Branche gemäß der Wirtschaftszweigsystematik. ${ }^{64}$ Diese Heterogenität sollte somit auch in einer Untersuchung zur

60 Aufgrund ihrer unternehmensinternen Patent- bzw. IP-Abteilungen mit entsprechend spezialisiertem Fachpersonal haben Großunternehmen, unabhängig von den Kostengesichtspunkten einer IPR-Nutzung, häufig gegenüber kleineren Unternehmen den Vorteil eines professionelleren IP-Managements. Dies versetzt größere Unternehmen eher in die Lage, IPRs nicht nur zum Schutz von Innovationen sondern auch als strategisches Instrument im Wettbewerb zu nutzen (etwa zur Blockade von Wettbewerbern, zur Verbesserung der Unternehmensreputation, zur Erfüllung einer Tauschmittelfunktion etc.). Vgl. hierzu Matthews, D. u. a. (2003), S. 52; Radauer, A. u. a. (2007), S. 28ff; Blind, K. u. a. (2006), S. 661ff.

61 Vgl. z.B. Kingston, W. (2000). Zur Senkung der hohen IPR-Durchsetzungskosten für KMU, schlägt Kingston, W. (2000), S. 11f, die Etablierung obligatorischer Schiedsgerichtsverfahren oder die Gründung eines Interessenverbandes patentierender KMU (eine sog. „Patent Defence Union“) auf freiwilliger Basis vor. Um kleinere Unternehmen gegen das Risiko von Durchsetzungsstreitigkeiten besser abzusichern, diskutieren Lanjouw, J. O. und Schankerman, M. (2004), S. 68f, dagegen die Möglichkeit zur effektiveren Ausgestaltung von Rechtsschutzversicherungen gegen Patentklagen.

62 Vgl. European Commission (2004), S. 13 und S. 52.

63 Vgl. Abschnitt 2.3.

64 Vgl. Kornhardt, U. und Kucera, G. (2003), S. 108ff; Dürig, W. u. a. (2004), S. 15ff. 
Rolle des Handwerks im Rahmen des gesamtwirtschaftlichen Konjunkturgeschehens durch eine getrennte Betrachtung von handwerklichen Gewerbegruppen berücksichtigt werden. Zweitens liegen den bisherigen Untersuchungen jährliche Werte zur Messung des langfristigen Konjunkturverlaufs im Handwerk zu Grunde. Zur Analyse von eher kurzfristigen Konjunkturschwankungen sind jedoch vor allem unterjährige Informationen von Interesse, weshalb im vorliegenden Fall auf vierteljährliche Beschäftigungs- und Umsatzdaten der Handwerksberichterstattung zurückgegriffen wird. Drittens setzt eine Beantwortung der Ausgangsfrage unter methodischen Gesichtspunkten zumindest näherungsweise die Aufdeckung von konjunkturellen Zyklen der Handwerkswirtschaft durch einen Ausschluss von anderweitigen Einflüssen voraus. Solche Einflüsse können saisonaler Natur sein oder eher mit längerfristigen Trendentwicklungen zusammenhängen, wie z.B. den strukturell bedingten Beschäftigungs- und Umsatzverlusten im Handwerk ab der Mitte der 90er Jahre. ${ }^{65}$ Eine Berücksichtigung dieser drei Punkte im Rahmen des vierten Einzelbeitrags liefert die Grundlage für eine neue Antwort auf die Frage, inwieweit das Handwerk eine konjunkturelle Stabilisatorfunktion besitzt und welche Folgerungen sich hieraus für die Wirtschaftspolitik ergeben.

Die Untersuchung zeigt, dass sich die Handwerkswirtschaft gemessen an der Umsatzentwicklung in einen konjunkturabhängigen und einen weniger konjunkturabhängigen Bereich teilt. Da ersterer umsatztechnisch überwiegt, spricht dies eher gegen eine generelle Stabilisatorfunktion des Handwerks. Hierunter fallen sowohl die in der Wirtschaftskrise 2008/2009 besonders stark betroffenen Handwerke für den gewerblichen Bedarf als auch das Bau- und Ausbaugewerbe. Die konsumnahen Gewerbegruppen des Handwerks sind im Vergleich hierzu deutlich weniger konjunkturabhängig. Das charakteristische Beschäftigungsverhalten im überwiegenden Teil der Handwerkswirtschaft spricht andererseits eher für eine konjunkturelle Stabilisatorfunktion. Denn im Vergleich zur Gesamtwirtschaft hält der Großteil der Handwerksbetriebe auch in konjunkturell schlechten Zeiten tendenziell länger an eigenen Mitarbeitern fest. In diesem Zusammenhang beleuchtet der vierte Einzelbeitrag auch die Hintergründe der in den einzelnen Gewerbegruppen des Handwerks unterschiedlich stark ausgeprägten Konjunkturabhängigkeit. Hierdurch wird deutlich, dass die Betroffenheit des Handwerks von wirtschaftlichen Krisen stets von der Struktur der jeweils aktuellen volkswirtschaftlichen Nachfrageschwäche abhängt. Dies ist der Tatsache geschuldet, dass die unterschiedliche Konjunkturabhängigkeit der einzelnen Handwerksbereiche im Wesentlichen ein Spiegelbild der gesamtwirtschaftlichen Nachfrageentwicklung ist. Konkret wird die konjunkturelle Entwicklung im Handwerk in erster Linie von der Baunachfrage, danach in abgeschwächter Form von der privaten Konsumnachfrage und erst mit einigem Abstand von der Nachfrage nach Ausrüstungsgütern bestimmt. Die schwankungsanfällige Exportnachfrage spielt hingegen für die Ausschläge der Handwerkskonjunktur nur eine untergeordnete Rolle. Bei der privaten Investitionsnachfrage handelt es sich ebenfalls um eine besonders volatile Verwendungskomponente des Bruttoinlandsprodukts, wodurch sich die höhere Konjunkturabhängigkeit der entsprechenden handwerklichen Gewerbegruppen (Bau, Ausbau und vor allem gewerblicher Bedarf)

65 Vgl. Lageman, B. u. a. (2005), S. 4ff. 
erklärt. Die private Konsumnachfrage ist dagegen in der Regel ein Zyklusdämpfer der gesamtwirtschaftlichen Konjunkturlage. Dadurch können die konsumnahen Handwerke auch eher stabilisierend wirken.

Auf Grundlage dieser Ergebnisse erklärt sich zunächst die Lage des Handwerks in der Wirtschaftskrise 2008/2009. Die Gesamtwirtschaft wurde in der Krise vor allem durch einen Exporteinbruch sowie durch deutliche Rückgänge der Nachfrage nach Ausrüstungsgütern und gewerblichen Bauten in Mitleidenschaft gezogen. Abgesehen davon blieb die Binnennachfrage - auch aufgrund erheblicher konjunkturpolitischer Eingriffe - weitgehend stabil. ${ }^{66}$ Die Handwerkswirtschaft wiederum ist insgesamt gesehen kaum exportabhängig. Des Weiteren spielen die vom Einbruch der Export- und Ausrüstungsnachfrage des Krisenjahrs 2009 besonders stark betroffenen Handwerke für den gewerblichen Bedarf für die Ausschläge der Handwerkskonjunktur eine weniger große Rolle als andere Handwerksbereiche. Die konjunkturelle Lage stellte sich daher im Krisenjahr 2009 in weiten Teilen des binnenmarktorientierten Handwerks vergleichsweise stabil dar. Zusammengefasst lässt sich damit auch die Ausgangsfrage nach der konjunkturellen Stabilisatorfunktion des Handwerks folgendermaßen beantworten: Gemessen an der Umsatzentwicklung besitzt das Handwerk keine generelle Stabilisatorfunktion. In Abhängigkeit vom Charakter der jeweils aktuellen konjunkturellen Gesamtkonstellation kann ein solches Phänomen jedoch durchaus begünstigt werden. Die diesbezüglichen Grundvoraussetzungen sind eine stabile Entwicklung der Inlandsnachfrage und die geringe Exportabhängigkeit des Handwerks. Ist die Konjunktursituation aufgrund einer schwachen Binnennachfrage ungünstig, wie z.B. im Zeitraum 2002 bis 2005, kann das Handwerk aber auch als Krisenverstärker wirken. Vor allem die konjunkturellen Bewegungen im Bausektor und in abgeschwächter Form diejenigen im Konsumgüterbereich bestimmen dabei, ob das Handwerk stabilisiert oder nicht. Demgegenüber spricht ein relativ beharrendes Beschäftigungsverhalten eines Großteils der Handwerkswirtschaft dafür, dass das Handwerk im Bereich des Arbeitsmarkts eine wichtige konjunkturelle Stabilisatorfunktion besitzt.

Mit den Ergebnissen des vierten Einzelbeitrags sind verschiedene Implikationen verbunden. Die Tatsache, dass das Handwerk nur dann als Konjunkturstabilisator wirken kann, wenn sich die Inlandsnachfrage entsprechend stabil entwickelt, zeigt zunächst, wie wichtig aus Sicht des Handwerks konkrete wirtschaftspolitische Maßnahmen zur Stärkung des Binnenmarkts sind. Gleichwohl dürfte die deutsche Wirtschaft auch zukünftig nicht umhin können, auf die Export- und Weltmarktorientierung als wichtigen Wachstumstreiber zu setzen. ${ }^{67}$ Eine etwaige Stabilisatorfunktion des kaum exportabhängigen Handwerks dürfte daher auch zukünftig bedeuten, dass die Handwerkswirtschaft von wichtigen Wachstumsbereichen der Volkswirtschaft weitgehend abgekoppelt bleibt. In den Betrieben und Organisationen des Handwerks sollten deshalb die Wogen der Weltkonjunktur stärker als bisher als lohnenswerte Chance be-

66 Zur Lage der deutschen Volkswirtschaft im Jahr 2009 siehe ifo (2009a), S. 29ff und ifo (2009b), S. 27ff.

67 Dies gilt trotz des Umstands, dass sich im Zuge der Wirtschaftskrise 2008/2009 eine intensive Debatte hinsichtlich der Vor- und Nachteile der überdurchschnittlichen Exportorientierung der deutschen Volkswirtschaft entwickelt hat. Vgl. hierzu z.B. Horn, G. u. a. (2010). 
griffen werden. Dies kann zum einen durch eine unmittelbare Ausweitung der handwerklichen Auslandsgeschäfte geschehen. ${ }^{68}$ Hinsichtlich der indirekten Exportimpulse wäre zum anderen eine stärkere Einbindung des Handwerks in die gesamtwirtschaftliche Wertschöpfungskette wichtig. Aufgrund der aufgezeigten konjunkturellen Stabilisatorfunktion des Handwerks im Bereich des Arbeitsmarkts sollten Handwerksbetriebe zudem bei Bedarf durch geeignete wirtschaftspolitische Maßnahmen in die Lage versetzt werden, ihre Beschäftigten auch in konjunkturell schwierigen Zeiten möglichst lange zu halten. ${ }^{69}$ Darüber hinaus setzt die Erfüllung einer solchen Funktion die Sicherstellung des Personalangebots voraus. Damit das Handwerk daher zukünftig in Zeiten des verschärften Fachkräftemangels eine beschäftigungsstabilisierende Funktion ausüben kann, sind entsprechende Anstrengungen seitens der Betriebe, der Handwerksorganisationen und der Politik gefragt.

\section{Literaturverzeichnis}

Abel, R. (2006): Innovationen in Kleinunternehmen: Wahrnehmung, Wirklichkeit und Wege, in: Abel, R., Bass, H. H. und Ernst-Siebert, R. (Hrsg.), Kleine und mittelgroße Unternehmen im globalen Innovationswettbewerb, München und Mering, S. 63-87.

Acs, Z. J. und Audretsch, D. B. (2005): Innovation and technological change, in: Acs, Z. J. und Audretsch, D. B. (Hrsg.), Handbook of entrepreneurship research. An interdisciplinary survey and introduction, New York, S. 55-79.

Amara, N., Landry, R. und Traoré, N. (2008): Managing the protection of innovations in knowledge-intensive business services, in: Research Policy, Jg. 37, Heft 9, S. 1530-1547.

Arundel, A. (2001): The relative effectiveness of patents and secrecy for appropriation, in: Research Policy, Jg. 30, Heft 4, S. 611-624.

Baldwin, J. und Gellatly, G. (2003): Innovation strategies and performance in small firms, Cheltenham, UK.

Blind, K., Cuntz, A., Köhler, F. und Radauer, A. (2009): Die volkswirtschaftliche Bedeutung geistigen Eigentums und dessen Schutzes mit Fokus auf den Mittelstand, Studie im Auftrag des Bundesministeriums für Wirtschaft und Technologie, in: http://www.bmwi.de/Dateien/Patentserver/PDF/volkswirtschaftliche-bedeutung-geistigen-eigentumsendbericht,property=pdf,bereich=bmwi,sprache=de,rwb=true.pdf (letzter Zugriff: 22.07.2011).

Blind, K., Edler, J., Frietsch, R. und Schmoch, U. (2006): Motives to patent: Empirical evidence from Germany, in: Research Policy, Jg. 35, Heft 5, S. 655-672.

BMWi (2010): Mit dem Patent zum Erfolg. Innovationsförderung für Unternehmen, Bericht zur Initiative SIGNO - Schutz von Ideen für die gewerbliche Nutzung, Bundesministerium für Wirtschaft und Technologie (BMWi), in: http://www.patentserver.de/Dateien/Patentserver/PDF/mit-dem-patent-zumerfolg,property=pdf,bereich=patentserver,sprache=de,rwb=true.pdf (letzter Zugriff: 19.07.2011).

68 Die Untersuchung von Müller, K. (2008), S. 33ff und S. 63f, hat gezeigt, dass in der Handwerkswirtschaft noch ein erhebliches Exportpotenzial besteht.

69 Die auch für Handwerksbetriebe erleichterte Möglichkeit zur Inanspruchnahme des Kurzarbeitergeldes im Rahmen des zweiten Konjunkturpakets während der Wirtschaftskrise 2008/2009 kann z.B. als entsprechende Maßnahme gewertet werden. 
BMWi (2011): Auf den Mittelstand setzen: Verantwortung stärken - Freiräume erweitern. Die Mittelstandsinitiative des Bundesministeriums für Wirtschaft und Technologie (BMWi), in: http://www.bmwi.de/BMWi/Redaktion/PDF/Publikationen/mittelstandsinitiative,property=pdf,bereich=bmwi,sprache=de,rwb=true.pdf (letzter Zugriff: 03.07.2011).

Borger, K. (2011): Mittelstandskonjunktur: Normalisierung hat begonnen, in: KfW-ifoMittelstandsbarometer: Mai 2011, in: http://www.kfw.de/kfw/de///I/Download_Center/Fachthemen/Research/PDFDokumente_Mittelstandsbarometer/KfW-ifo-Mittelstandsbarometer_2011-05_.pdf (letzter Zugriff: 21.07.2011).

Bridge, S., O'Neill, K. und Martin, F. (2009): Understanding enterprise. Entrepreneurship and small business, third edition, Basingstoke.

Cohen, W. (1995): Empirical studies of innovative activity, in: Stoneman, P. (Hrsg.), Handbook of the economics of innovation and technological change, Oxford S. 182-264.

Cohen, W. M., Nelson, R. R. und Walsh, J. P. (2000): Protecting their intellectual assets: appropriability conditions and why U.S. manufacturing firms patent (or Not), in: NBER Working Papers Series, Heft 7552.

Curran, J. und Blackburn, R. A. (2001): Researching the small enterprise, London.

Davidsson, P., Lindmark, L. und Olofsson, C. (1999): SMEs and job creation during a recession and recovery, in: Acs, Z. J., Carlsson, B. und Karlsson, C. (Hrsg.), Entrepreneurship, small and medium-sized enterprises and the macroeconomy, Cambridge, S. 286-309.

Dosi, G., Marengo, L. und Pasquali, C. (2006): How much should society fuel the greed of innovators? On the relations between appropriability, opportunities and rates of innovation, in: Research Policy, Jg. 35, Heft 8, S. 1110-1121.

Dürig, W., Lagemann, B., Rothgang, M., Trettin, L. und Welter, F. (2004): Determinanten des Strukturwandels im deutschen Handwerk, Band I, Rheinisch-Westfälisches Institut für Wirtschaftsforschung, Essen.

Erixon, F. (2009): SMEs in Europe: taking stock and looking forward in: European View, Jg. 8, Heft 2, S. 293-300.

European Commission (2004): SMEs and cooperation. Observatory of European SMEs 2003, No. 5, in: http://ec.europa.eu/enterprise/policies/sme/files/analysis/doc/smes_observatory_2003_report5_en.pdf (letzter Zugriff: 01.08.2011).

European Commission (2005): The new SME definition. User guide and model declaration, European Commission, Enterprise and Industry Publications, in: http://ec.europa.eu/enterprise/policies/sme/files/sme_definition/sme_user_guide_en.pdf (letzter Zugriff: 06.07.2011).

European Commission (2008): “Think Small First”. A “Small Business Act” for Europe. Communication from the Commission to the Council, the European Parliament, the European Economic and Social Committee and the Committee of the Regions, in: http://eur-lex.europa.eu/LexUriServ/LexUriServ.do?uri=COM:2008:0394:FIN:en:PDF (letzter Zugriff: 03.07.2011).

Evangelista, R. (2000): Sectoral patterns of technological change in services, in: Economics of Innovation and New Technology, Jg. 9, Heft 3, S. 183-221. 
Fendel, R. und Frenkel, M. (1998): Do small and medium-sized enterprises stabilize employment? Theoretical considerations and evidence from Germany, in: Zeitschrift für Wirtschafts- und Sozialwissenschaften, Jg. 118, S. 163-184

Filippetti, A. und Archibugi, D. (2011): Innovation in times of crisis: National Systems of Innovation, structure, and demand, in: Research Policy, Jg. 40, Heft 2, S. 179-192.

Flüter-Hoffmann, C. und Stettes, O. (2011): Neue Balance zwischen betrieblicher Flexibilität und Stabilität - Ergebnisse einer repräsentativen IW-Befragung, in: IW-Trends Vierteljahresschrift zur empirischen Wirtschaftsforschung aus dem Institut der deutschen Wirtschaft Köln, Heft 2011/1, S. 3-18.

Frank, H., Lueger, M. und Korunka, C. (2010): Innovative Kleinunternehmen: Eine qualitative Systemanalyse, in: Zeitschrift für KMU und Entrepreneurship (ZfKE), Jg. 58, Heft 1, S. 1-30.

Gamble, J. (2001): Modelling the invisible: the pedagogy of craft apprenticeship, in: Studies in Continuing Education, Jg. 23, Heft 2, S. 185-200.

Gamble, J. (2004): Retrieving the general from the particular. The structure of craft knowledge, in: Muller, J., Davies, B. und Morais, A. (Hrsg.), Reading Bernstein, Researching Bernstein, London und New York, S. 189-203.

Geroski, P. (1995): Markets for technology: knowledge, innovation and appropriability, in: Stoneman, P. (Hrsg.), Handbook of the economics of innovation and technological change, Oxford, S. 90-131.

Geroski, P. A. und Walters, C. F. (1995): Innovative activity over the business cycle, in: The Economic Journal, Jg. 105, S. 916-928.

González-Álvarez, N. und Nieto-Antolín, M. (2007): Appropriability of innovation results: an empirical study in spanish manufacturing firms, in: Technovation, Jg. 27, Heft 5, S. 280295.

Granstrand, O. (1999): The economics and management of intellectual property: towards intellectual capitalism, Cheltenham, UK.

Greenhalgh, C. und Rogers, M. (2007): The value of intellectual property rights to firms and society, in: Oxford Review of Economic Policy, Jg. 23, Heft 4, S. 541-567.

Guellec, D. und van Pottelsberghe de la Potterie, B. (2007): The economics of the European patent system. IP policy for innovation and competition, Oxford

Hall, M., Oppenheim, C. und Sheen, M. (2003): Barriers to the use of patent information in SMEs, in: Blackburn, R. A. (Hrsg.), Intellectual property and innovation management in small firms, London und New York, S. 144-160.

Harabi, N. (1995): Appropriability of technical innovations. An empirical analysis, in: Research Policy, Jg. 24, Heft 6, S. 981-992.

Hollenstein, H. (2003): Innovation modes in the Swiss service sector: a cluster analysis based on firm-level data, in: Research Policy, Jg. 32, Heft 5, S. 845-863.

Horn, G., Sturn, S. und Treeck, T. (2010): Die Debatte um die deutsche Exportorientierung, in: Wirtschaftsdienst - Zeitschrift für Wirtschaftspolitik, Jg. 90, Heft 1, S. 22-28.

Hurmelinna-Laukkanen, P. und Puumalainen, K. (2007): Nature and dynamics of appropriability: strategies for appropriating returns on innovation, in: R\&D Management, Jg. 37, Heft 2, S. 95-112. 
ifo (2009a): Im Sog der Weltrezession. Gemeinschaftsdiagnose Frühjahr 2009, in: ifo Institut für Wirtschaftsforschung, ifo Schnelldienst, Jg. 62, Heft 8/2009.

ifo (2009b): Zögerliche Belebung - steigende Staatsschulden. Gemeinschaftsdiagnose Herbst 2009, in: ifo Institut für Wirtschaftsforschung, ifo Schnelldienst, Jg. 62, Heft 20/2009.

IW-Köln (2010): Handwerk - Fast ohne Schrammen durch die Krise, in: Informationsdienst des Instituts der deutschen Wirtschaft, Köln, Jg. 36, Heft 17, S. 4-5.

Jensen, M. B., Johnson, B., Lorenz, E. und Lundvall, B. Å. (2007): Forms of knowledge and modes of innovation, in: Research Policy, Jg. 36, Heft 5, S. 680-693.

Jensen, P. H. und Webster, E. (2006): Firm size and the use of intellectual property rights, in: The Economic Record, Jg. 82, Heft 256, S. 44-55.

Jong de, J. und Marsili, O. (2006): The fruit flies of innovations: A taxonomy of innovative small firms, in: Research Policy, Jg. 35, Heft 2, S. 213-229.

KfW, Creditreform, IfM, RWI und ZEW (2010): Konjunkturelle Stabilisierung im Mittelstand - aber viele Belastungsfaktoren bleiben, MittelstandsMonitor 2010 - Jährlicher Bericht zu Konjunktur- und Strukturfragen kleiner und mittlerer Unternehmen, Frankfurt am Main.

Kingston, W. (2000): Enforcing small firms' patent rights. Research report funded by the European Commission. Trinity College, Dublin, Ireland, in: ftp://ftp.cordis.europa.eu/pub/innovation-policy/studies/studies_enforcing_firms_patent_rights.pdf (letzter Zugriff: 20.07.2011).

Kitching, J. und Blackburn, R. A. (2003): Innovation, intellectual property and informality: evidence from a study of small enterprises and some implications for policy, in: Blackburn, R. A. (Hrsg.), Intellectual property and innovation management in small firms, London und New York, S. 16-34.

Kleinknecht, A. H. (1987): Measuring R\&D in small firms: how much are we missing?, in: The Journal of Industrial Economics, Jg. 36, Heft 2, S. 253-256.

Kleinknecht, A. H. (1991): More evidence on the undercounting of small firm R\&D, in: Research Policy, Jg. 20, Heft 6, S. 579-587.

Kornhardt, U. und Kucera, G. (2003): Investitionsverhalten im Handwerk: Ursachen für die Investitionsschwäche im Handwerk seit Mitte der 90er Jahre, Göttinger handwerkswirtschaftliche Studien, Nr. 68, Duderstadt.

Krämer, W. (2003): Mittelstandsökonomik. Grundzüge einer umfassenden Analyse kleiner und mittlerer Unternehmen, München.

Lageman, B., Dürig, W. und Trettin, L. (2005): Determinanten des Strukturwandels im Handwerk, in: Bundesarbeitsblatt, Heft 7-2005, S. 4-16.

Lahner, J. (2004): Innovationsprozesse im Handwerk, Göttinger handwerkswissenschaftliche Studien, Nr. 69, Duderstadt.

Lahner, J. (2008): Innovative Kleinunternehmen - systematisch unterschätzt oder nur übersehen?, in: RegioPol - Zeitschrift für Regionalwirtschaft, Jg. 2, S. 53-61.

Lanjouw, J. O. und Schankerman, M. (2004): Protecting intellectual property rights: Are small firms handicapped?, in: The Journal of Law \& Economics, Jg. 47, Heft 1, S. 45-74.

Leiponen, A. und Byma, J. (2009): If you cannot block, you better run: Small firms, cooperative innovation, and appropriation strategies, in: Research Policy, Jg. 38, Heft 9, S. 1478-1488. 
Levin, R. C., Klevorick, A. K., Nelson, R. R. und Winter, S. G. (1987): Appropriating the returns from industrial research and development, in: Brookings Papers on Economic Activity, Jg. 3, S. 783-831.

Lundvall, B.-Å. und Borrás, S. (2005): Science, technology, and innovation policy, in: Fagerberg, J., Mowery, D. C. und Nelson, R. R. (Hrsg.), The Oxford handbook of innovation, Oxford, S. 599-631.

Macdonald, S. (2003): Bearing the Burden: Small Firms and the Patent System, in: Journal of Information, Law and Technology (JILT), Heft 1/2003, in: http://www2.warwick.ac.uk/fac/soc/law/elj/jilt/2003_1/macdonald (letzter Zugriff: 20.07.2011).

Malerba, F. (2005): Sectoral systems. How and why innovation differs across sectors, in: Fagerberg, J., Mowery, D. C. und Nelson, R. R. (Hrsg.), The Oxford handbook of innovation, Oxford, S. 380-406.

Malerba, F. und Orsenigo, L. (1997): Technological regimes and sectoral patterns of innovative activities, in: Industrial and Corporate Change, Jg. 6, Heft 1, S. 83-117.

Matthews, D., Pickering, J. und Kirkland, J. (2003): A strategic approach to managing intellectual property, in: Blackburn, R. A. (Hrsg.), Intellectual property and innovation management in small firms, London and New York, S. 35-54.

Maußner, A. (1994): Konjunkturtheorie, Berlin.

Mazzarol, T. und Reboud, S. (2005): Customers as predictors of rent returns to innovation in small firms: an exploratory study, in: International Journal of Entrepreneurship and Innovation Management, Jg. 5, Heft 5-6, S. 483-494.

Mazzarol, T. und Reboud, S. (2009): The strategy of small firms. Strategic management and innovation in the small firm, Cheltenham, UK.

Momm, H.-J. (1983): Konjunkturprognosen im Handwerk. Systeme der Früherkennung von Konjunkturbetroffenheiten in ausgewählten Handwerkszweigen, Göttinger handwerkswirtschaftliche Studien, Nr. 32, Göttingen.

Moscarini, G. und Postel-Vinay, F. (2009): Large employers are more cyclically sensitive, in: NBER Working Paper Series, Working Paper 14740.

Müller, K. (2008): Auslandsgeschäfte im Handwerk, Göttinger handwerkswirtschaftliche Studien, Nr. 76, Duderstadt.

Nieto, M. und Pérez-Cano, C. (2004): The influence of knowledge attributes on innovation protection mechanisms, in: Knowledge and Process Management, Jg. 11, Heft 2, S. 117126.

Nooteboom, B. (1994): Innovation and diffusion in small firms: Theory and evidence, in: Small Business Economics, Jg. 6, Heft 5, S. 327-347.

OECD und Eurostat (2005): Oslo Manual. The measurement of scientific and technological activities. Guidelines for collecting and interpreting innovation data, third edition, in: http://browse.oecdbookshop.org/oecd/pdfs/free/9205111e.pdf (letzter Zugriff: 13.07.2011), Paris.

Olander, H., Hurmelinna-Laukkanen, P. und Mähönen, J. (2009): What's small size got to do with it? Protection of intellectual assets in SMEs, in: International Journal of Innovation Management, Jg. 13, Heft 3, S. 349-370.

Ortega-Argilés, R., Vivarelli, M. und Voigt, P. (2009): R\&D in SMEs: a paradox?, in: Small Business Economics, Jg. 33, S. 3-11. 
Pavitt, K. (1984): Sectoral patterns of technical change: towards a taxonomy and a theory, in: Research Policy, Jg. 13, Heft 6, S. 343-373.

Peneder, M. (2010): Technological regimes and the variety of innovation behaviour: Creating integrated taxonomies of firms and sectors in: Research Policy, Jg. 39, Heft 3, S. 323-334.

Penrose, E. (1959): The theory of the growth of the firm, third edition, published 1995, Oxford.

Pleschak, F., Sabisch, H. und Wupperfeld, U. (1994): Innovationsorientierte kleine Unternehmen, Wiesbaden.

Polanyi, M. (1958): Personal knowledge. Towards a post-critical philosophy, Paperback edition published 1974, Chicago.

PRO INNO Europe (2007): A memorandum on removing barriers for a better use of IPR by SMEs, A Report for the Directorate-General for Enterprise and Industry (European Commission) by an IPR Expert Group, Paper No. 3, in: http://www.proinnoeurope.eu/publications (letzter Zugriff: 19.07.2011).

Radauer, A., Streicher, J. und Ohler, F. (2007): Benchmarking national and regional support services for SMEs in the field of intellectual and industrial property, Final benchmarking report on behalf of the European Commission, Austrian Institute for SME Research, in: http://www.proinno-europe.eu/publications (letzter Zugriff: 19.07.2011).

Rammer, C., Czarnitzki, D. und Spielkamp, A. (2009): Innovation success of non-R\&Dperformers: substituting technology by management in SMEs, in: Small Business Economics, Jg. 33, Heft 1, S. 35-58.

Rantakyrö, L. (2005): Knowledge management in small firms, in: Global Business and Economics Review, Jg. 7, Heft 2/3, S. 214-225.

Reed, R. und DeFillippi, R. J. (1990): Causal ambiguity, barriers to imitation, and sustainable competitive advantage, in: Academy of Management Review, Jg. 15, Heft 1, S. 88-102.

Rothwell, R. (1983): Innovation and firm size: a case for dynamic complementarity; or, is small really so beautiful?, in: Journal of General Management, Jg. 8, Heft 3, S. 5-25.

Scheidegger, E. und Brunetti, A. (2003): Warum eine KMU-Politik?, in: Die Volkswirtschaft. Das Magazin für Wirtschaftspolitik, Jg. 76, Heft 6, S. 4-7.

Schmidt, K.-H. (1975): Die Konjunkturabhängigkeit des Handwerks, in: Aßmann, K. und Schmidt, K.-H. (Hrsg.), Die Konjunkturabhängigkeit der Klein- und Mittelbetriebe, Göttinger handwerkswirtschaftliche Studien, Nr. 26, Göttingen, S. 1-171.

Schumpeter, J. A. (1934): Theorie der wirtschaftlichen Entwicklung. Eine Untersuchung über Unternehmergewinn, Kapital, Kredit, Zins und den Konjunkturzyklus, 8. Auflage, erschienen 1993 bei Duncker \& Humblot, unveränderter Nachdruck der 1934 erschienenen 4. Auflage, Berlin.

Schumpeter, J. A. (1942): Kapitalismus, Sozialismus und Demokratie, Titel der Originalausgabe von 1942: Capitalism, Socialism and Democracy, 6. Auflage, erschienen 1987 im Francke Verlag, Tübingen.

Scotchmer, S. (2004): Innovation and Incentives, Cambridge, Massachusetts.

Semlinger, K. (2007): Innovationshemmnis "Kundennähe"? - Zur Notwendigkeit einer nachfrageseitigen Ergänzung der kleinbetriebsorientierten Innovationsförderung, in: Zeitschrift für KMU und Entrepreneurship, Jg. 55, Heft 3, S. 147-166.

Sennett, R. (2008): The craftsman, New Haven and London. 
Storey, D. J. (1994): Understanding the small business sector, London.

Storey, D. J. (2005): Entrepreneurship, small and medium sized enterprises and public policies, in: Acs, Z. J. und Audretsch, D. B. (Hrsg.), Handbook of entrepreneurship research. An interdisciplinary survey and introduction, New York, S. 473-511.

Symeonidis, G. (1996): Innovation, firm size and market structure: Schumpeterian hypotheses and some new themes, in: OECD Economics Department Working Paper, Heft 161, OECD Publishing, in: http://www.oecdilibrary.org/docserver/download/fulltext/5lgsjhvj86f0.pdf?expires=1310309030\&id=id\&accname=guest\&c hecksum=4E940871D4113E7761913E452367A568 (letzter Zugriff: 10.07.2011).

Teece, D. J. (1986): Profiting from technological innovation: Implications for integration, collaboration, licensing and public policy, in: Research Policy, Jg. 15, Heft 6, S. 285-305.

Teece, D. J. (2003): The strategic management of technology and intellectual property, in: Faulkner, D. O. und Campbell, A. (Hrsg.), The Oxford Handbook of Strategy; Volume I: A Strategy Overview and Competitive Strategy, Oxford, S. 132-166.

Teece, D. J. (2006): Reflections on “Profiting from Innovation”, in: Research Policy, Jg. 35, Heft 8, S. 1131-1146.

Varum, C. A. und Rocha, V. C. (2011): Employment and SMEs during crises, in: Small Business Economics, Online First: DOI: 10.1007/s11187-011-9343-6 (im Erscheinen).

von Tunzelmann, N. und Acha, V. (2005): Innovation in "low-tech" industries, in: Fagerberg, J., Mowery, D. C. und Nelson, R. R. (Hrsg.), The Oxford handbook of innovation, Oxford, S. 407-432.

Welter, F. (2003): Strategien, KMU und Umfeld. Handlungsmuster und Strategiegenese in kleinen und mittleren Unternehmen, Berlin.

WIPO (2003): Intellectual property (IP) rights and innovation in small and medium-sized enterprises, World Intellectual Property Organization (WIPO), in:

http://www.wipo.int/export/sites/www/sme/en/documents/pdf/iprs_innovation.pdf (letzter Zugriff: 19.07.2011).

WIPO (A): Understanding Industrial Property, World Intellectual Property Organization (WIPO), Publication No. 895(E), in: http://www.wipo.int/freepublications/en/intproperty/895/wipo_pub_895.pdf (letzter Zugriff: 20.07.2011).

WIPO (B): Understanding Copyright and related rights, World Intellectual Property Organization (WIPO), Publication No. 909(E), in:

http://www.wipo.int/freepublications/en/intproperty/909/wipo_pub_909.pdf (letzter Zugriff: 20.07.2011).

Wynarczyk, P., Watson, R., Storey, D., Short, H. und Keasey, K. (1993): Managerial labour markets in small and medium-sized enterprises, London.

Zander, U. und Kogut, B. (1995): Knowledge and the speed of the transfer and imitation of organizational capabilities: An empirical test, in: Organization Science, Jg. 6, Heft 1, S. 76-92.

ZDH (2009a): Konjunkturbericht 1/2009, Kein Einbruch der Handwerkskonjunktur - Aber starke Verlangsamung und gedämpfte Erwartungen, Zentralverband des Deutschen Handwerks (ZDH), Berlin. 
ZDH (2009b): Konjunkturbericht 2/2009, Handwerkskonjunktur spürbar belebt - Starke Unterschiede zwischen den Branchen, Zentralverband des Deutschen Handwerks (ZDH), Berlin.

Zimmermann, V. (2010): Innovation und Konjunktur, in: KfW-Research, Reihe "Standpunkt", Nr 4., in: http://kfw.de/kfw/de///II/Download_Center/Fachthemen/Research/PDFDokumente_Standpunkt/Standpunkt,_Ausgabe_4,_Juni_2010.pdf (letzter Zugriff: 19.07.2011). 


\section{Kapitel II}

Kleinunternehmen und der Schutz von Innovationen benachteiligt oder einfach anders? 


\section{Einleitung}

Die Wettbewerbsfähigkeit von Unternehmen und Volkswirtschaften bestimmt sich maßgeblich durch die Generierung, Kommerzialisierung und Verbreitung von Innovationen. Dem Schutz geistigen Eigentums durch Patente und andere „Intellectual Property Rights“ (IPRs) wird hierbei eine besondere Rolle beigemessen. Dahinter steht die Annahme, dass Innovatoren mit Hilfe von IPRs eine bessere Aneignung von Innovationserträgen gelingt, woraus konkrete Innovationsanreize resultieren und ein entsprechendes Marktversagen abgebaut wird (Greenhalgh / Rogers 2007, 544 f.). Vor dem Hintergrund eines zunehmend strategisch orientierten IPR-Einsatzes (Burr et al. 2007), neueren Entwicklungen wie der „open-science“-Bewegung (David 2004) und vertieften Erkenntnissen darüber, unter welchen Bedingungen Unternehmen von ihren Innovationen profitieren können (Teece 2002), wird diese theoretische Begründung für die Existenz von IPRs jedoch auch kritisch hinterfragt (Dosi et al. 2006a; Dosi et al. 2006b; Nelson 2006). Nach Suzanne Scotchmer gehen dennoch viele Diskussionen über den innovationsförderlichen Effekt verbesserter Aneignungsbedingungen (,appropriability“'1) von der Grundannahme aus, dass der IPR-Schutz bereits die Lösung darstellt. Sie hält es stattdessen für angebracht, anstatt mit der Lösung zunächst mit dem Problem (d.h. die Schwierigkeit von Innovationen zu profitieren und hieraus resultierende Anreizprobleme) zu beginnen (Scotchmer 2004, 39 ff. und 259 ff.).

Gerade im Falle von Einschätzungen zur Relevanz von IPRs für kleine und mittlere Unternehmen (KMU) scheint das von Scotchmer beschriebene Phänomen charakteristisch zu sein. Denn oftmals besteht die Sorge, dass die unterdurchschnittliche Nutzung des IPR-Systems durch KMU mit einer geringeren Innovationstätigkeit in kleineren Unternehmen einhergeht (Iversen 2003, 13 ff.). Die Förderung einer intensiveren Nutzung formeller Schutzrechte wird in der Folge als Möglichkeit begriffen, das „appropriability“-Problem in KMU zu lösen (WIPO 2003, 2). Unterstützung findet diese Annahme bereits durch die klassische Zusammenstellung der KMU-spezifischen Vor- und Nachteile im Innovationsprozess von Rothwell (1983), wonach kleinere Unternehmen mit Blick auf den Patentschutz benachteiligt sind. Hierfür sprechen Befunde, wonach z.B. hohe Kosten der Anmeldung und Durchsetzung formeller Schutzrechte sowie die Komplexität der in Patentdatenbanken gespeicherten Informationen dazu führen, dass KMU aufgrund größenbedingter Defizite einen geringeren Nutzen aus dem IPR-System ziehen können (Hall et al. 2003; Lanjouw / Schankerman 2004; Macdonald 2003). In der förderpolitischen Praxis leiten sich vor diesem Hintergrund zahlreiche KMU-relevante Unterstützungsmaßnahmen auf nationaler und internationaler Ebene ab (Radauer et al. 2007; Blind et al. 2009, 94 ff.). Mit Blick auf das am Anfang stehende „appropriability“-Problem wird hierbei jedoch selten nach der tatsächlichen Relevanz von IPRs für die Aneignung von Innovationserträgen in kleineren Unternehmen gefragt. Dies verwundert insofern, da betriebliche Innovationsprozesse und die damit einhergehende Wahl adäquater

\footnotetext{
"Appropriability of innovations summarizes the possibilities of protecting innovations from imitation and of reaping profits from innovative activities” (Malerba 2002, 252)
} 
Innovationstrategien in KMU spezifische Besonderheiten aufweisen (Baldwin / Gellatly 2003; Meyer 2001). Nicht zuletzt der Kleinunternehmenssektor (weniger als 50 Beschäftigte) ist hierbei angesprochen, was sich beispielsweise in einer begrenzten finanziellen und organisatorischen Ressourcenbasis, möglichen Vorteilen der kleineren Unternehmensgröße (Flexibilität, Kundennähe usw.), der bestimmenden Rolle der Unternehmerpersönlichkeit oder einem deutlich geringeren Formalisierungsgrad der Innovationstätigkeit niederschlägt (Abel 2006; Lahner 2008). Von vornherein kann daher nicht ausgeschlossen werden, dass in kleineren Unternehmen die Aneignung von Innovationserträgen ebenfalls charakteristische Besonderheiten aufweist.

Trotz der vorhandenen Evidenz für eine Benachteiligung von KMUs im IPR-System muss daher festgestellt werden, dass eine abschließende Antwort auf die übergreifende Frage, warum IPRs durch kleinere Unternehmen weniger genutzt werden, weiterhin aussteht. Der vorliegende Aufsatz setzt hier an, indem er das „appropriability“-Problem aus einer KMUPerspektive diskutiert, erste Antworten gibt und nicht zuletzt den Weg für weiterführende Forschungsanstrengungen aufzeigt. Der Beitrag gliedert sich wie folgt. Kapitel II umreißt den theoretischen und empirischen Untersuchungsrahmen. Kapitel III liefert die Ergebnisse einer empirischen Auswertung bezüglich grundlegender Einflussfaktoren auf die Nutzung von Aneignungsmechanismen. Vor diesem Hintergrund schließt Kapitel IV mit einem Fazit und einer ausführlichen Diskussion der Ergebnisse.

\section{Untersuchungsrahmen: Das „appropriability“-Problem}

\section{Theoretische Grundlagen}

Erste Grundzüge des „appropriability“-Problems wurden von Schumpeter (1942) beschrieben. Demnach würde im Zustand vollkommener Konkurrenz die Innovationsrate gering ausfallen, was letztlich auf die fehlende Möglichkeit zur Aneignung von Innovationserträgen zurückzuführen sei. Nur die Aussicht auf (zeitweise) Monopolgewinne würde Unternehmern den Anreiz zur Generierung von Innovationen geben, was in der Summe zum Prozess der „schöpferischen Zerstörung“ führe. Arrow (1962) und Nelson (1959) lieferten ebenfalls grundlegende Beiträge, indem sie mangelnde Innovationsanreize mit ökonomischen Eigenschaften von Wissensgütern in Verbindung brachten. Innovationswissen weise demnach Eigenschaften eines öffentlichen Gutes auf, weshalb eine Ausschließbarkeit, wenn überhaupt, nur teilweise möglich ist. Aufgrund von Externalitäten müsse eine Aneignung von Innovationserträgen daher immer unvollständig bleiben, was in der Folge zu einer Unterinvestition in Innovationsaktivitäten führt. Der Sinn des IPR-Schutzes besteht folglich darin, eine höhere Aneignung von Innovationserträgen zu erlauben und Innovationsanreize zu setzen.

Neben IPRs nutzen Unternehmen in der Praxis jedoch eine Vielzahl von Aneignungsmechanismen, um von eigenen Innovationen profitieren zu können. Die dahinter stehende Logik legte erstmals Teece (1986) dar. Demnach beeinflusst neben dem technologischen Paradigma 
einer Branche und der Zugriffsmöglichkeit auf komplementäre Güter ${ }^{2}$ vor allem das branchen- bzw. unternehmensspezifische Aneignungsregime die Höhe des Innovationserfolgs. Dessen Stärke bestimmt sich nach Teece durch den Grad der Replizierbarkeit einer Innovation und dem Wirkungsgrad von IPRs als Imitationsschutz. Ein Aneignungsregime wäre dann als schwach zu bezeichnen, wenn eine Innovation aufgrund einer hohen Kodifizierbarkeit des relevanten Wissens leicht nachvollziehbar ist und IPRs entweder nicht verfügbar oder ineffektiv sind (Teece 1986; Teece 2002, 16 ff. ). Daran anknüpfend verdeutlichen HurmelinnaLaukkanen / Puumalainen (2007) die Zusammenhänge zwischen verschiedenen Aneignungsmechanismen (vgl. Abb. 1). Neben IPRs kommen demnach weitere institutionelle Absicherungen wie Geheimhaltungsvereinbarungen, Ansätze des Personalmanagements zur Steigerung der Mitarbeiterbindung oder Strategien, die unter die Kategorien zeitlicher Vorsprung und Geheimhaltung fallen, als Aneignungsmechanismen in Betracht. Umso mehr die zu schützende Innovation zudem auf implizitem Erfahrungswissen („tacit knowledge“) basiert, das in den Arbeitsroutinen des Unternehmens eingebettet ist, desto größer sei die Wirkung eines natürlichen Imitationsschutzes. Wissen nimmt somit nicht nur die Rolle des Schutzgegenstandes ein, sondern kann selbst als Aneignungsmechanismus fungieren (HurmelinnaLaukkanen / Puumalainen 2007, 96 ff.).

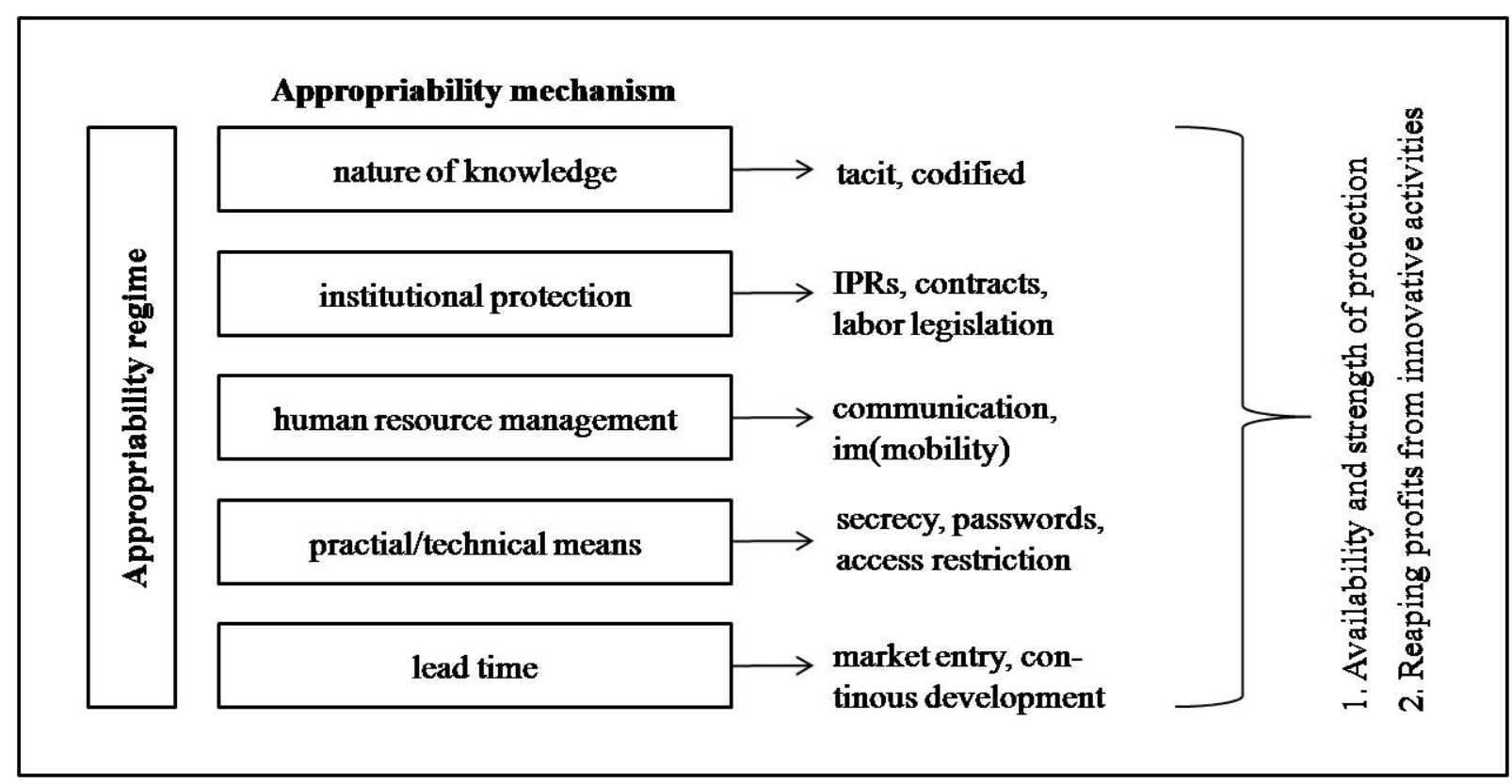

Quelle: Darstellung auf Grundlage von Hurmelinna-Laukkanen / Puumalainen (2007), 98.

\section{Abbildung 1: Das Aneignungsregime mit seinen Bestandteilen}

Aneignungsmechanismen erfüllen dabei stets zwei Funktionen (vgl. Abb. 1). Erstens müssen sie verfügbar und wirksam sein (Imitationsschutz). Zweitens bestimmt sich deren Effizienz nicht zuletzt durch den Beitrag zum Innovationserfolg (Hurmelinna-Laukkanen 2009, 285). Hierbei ist zu beachten, dass ein zu hoher Grad der Schutzwirkung auch schädlich

2 Ergänzende Fähigkeiten und Ressourcen, z.B. im Produktions-, Vertriebs- oder Marketingbereich. 
für den Innovationserfolg sein kann. Externe Wissensflüsse, z.B. in Form von Netzwerken, könnten hierdurch behindert werden, welche ansonsten positiv zum Innovationserfolg beigetragen hätten (Hurmelinna et al. 2007). Der anzustrebende Ausgleich zwischen Wissensaustausch und Wissensschutz führt zudem dazu, dass der Einsatz von Aneignungsmechanismen keine Maximierungs- sondern eine Optimierungsaufgabe darstellt. Die verschiedenen Aneignungsmechanismen müssen sich daher auch keineswegs gegenseitig ausschließen. Es ist z.B. denkbar, dass sich die Vorteile des Patentschutzes und der Geheimhaltungsstrategie erst durch deren gleichzeitige Nutzung zum Schutz von verschiedenen Komponenten einer Innovation bzw. durch deren Einsatz an verschiedenen Punkten im Lebenszyklus einer Innovation ergeben (Bosworth / Webster 2006, 89 f.).

\section{Empirische Literatur}

Die grundlegenden Studien von Levin et al. (1987) und Cohen et al. (2000) verdeutlichten, dass in den meisten Branchen der Patentschutz für innovierende Unternehmen im Vergleich zu anderen Aneignungsmechanismen nur von begrenzter Bedeutung ist. Stattdessen erschienen oftmals der zeitliche Vorsprung, die Geheimhaltungsstrategie und komplementäre Fähigkeiten im Vertrieb-, Service- und Produktionsbereich als deutlich wichtigere Aneignungsmechanismen. Produktinnovatoren schätzten besonders den zeitlichen Vorsprung als wichtig ein, wohingegen Prozessinnovatoren eher die Geheimhaltung als wirksam betrachteten. Für Produktinnovationen waren zudem Patente relativ gesehen wichtiger. Als Gründe nicht zu patentieren wurden in beiden Untersuchungen am häufigsten der Zwang zur Veröffentlichung der Patentschrift, die damit verbundene Sorge einer Umgehung der patentierten Erfindung und der mangelnde Neuheitsgrad von Erfindungen genannt. Die mit der Anmeldung und Durchsetzung von Patenten verbundenen Kosten waren vor allem für kleinere Unternehmen ein Grund auf diese zu verzichten (Cohen et al. 2000, 15 f.).

Bei einer Sichtung der allgemeinen empirischen Literatur ist die Konzentration auf bestimmte Aneignungsmechanismen (etwa Patente) auffällig. Andere Mechanismen - z.B. weitere IPRs oder die Rolle des „tacit knowledge“ - haben eine weitaus geringere Beachtung erfahren. Eine Ausnahme bildet die Studie von González-Álvarez / Nieto-Antolín (2007). Demnach setzen Unternehmen, die vor allem explizites Wissen („codified knowledge“) im Innovationsprozess nutzen und größer sind, eher auf den Patentschutz. Unternehmen, die sich hingegen durch eine hohe Bedeutung des impliziten Wissens auszeichnen, würden hingegen eher zur Geheimhaltung tendieren. Dies dürfte unter anderem darauf zurückzuführen sein, dass in größeren Unternehmen oftmals F\&E-Abteilungen vorhanden sind, was wiederum zu einem stärkeren Formalisierungsgrad der dortigen Innovationsprozesse führt. Des Weiteren steht in vielen Untersuchungen nur die Wirkung des Imitationsschutzes im Vordergrund. Die zweite Funktion eines Aneignungsmechanismus - der Beitrag zum Innovationserfolg - wurde deutlich seltener untersucht. Eine Ausnahme bildet hier die Studie von HurmelinnaLaukkanen et al. (2008). Nur für inkrementelle Innovatoren konnte in diesem Fall ein positiver Zusammenhang zwischen der Stärke des Aneignungsregimes und dem Innovationserfolg nachgewiesen werden. Angesichts der Tatsache, dass formelle Schutzrechte wie das Patent für 
inkrementelle Innovatoren weniger verfügbar sein dürften als für radikale Innovatoren, deuten diese Ergebnisse somit darauf hin, dass sich Aneignungsstrategien vor allem auch in Abhängigkeit vom Neuheitsgrad einer Innovation unterscheiden. ${ }^{3}$

Eine Literaturrecherche zeigt zudem, dass die Aneignung von Innovationserträgen in kleineren Unternehmen und damit einhergehend die Relevanz von IPRs für die Innovationstätigkeit in KMU bisher nicht systematisch untersucht wurde. Vor allem ist in diesem Zusammenhang wenig über Kleinunternehmen bekannt. Verschiedene Autoren mahnen daher einen entsprechenden Forschungsbedarf an (etwa Blackburn 2003 und Greenhalgh / Rogers 2007, 563 f.). Bei den vorliegenden Arbeiten ist hinsichtlich der Methodik zwischen einem statistisch-ökonometrischen Vorgehen, bei welchem sich der Thematik über das quantifizierbare Merkmal der Unternehmensgröße angenähert wird, und einem eher qualitativ gelagerten Vorgehen der angewandten Mittelstandsforschung, das die Besonderheiten kleinerer Unternehmen stärker in den Blick rücken lässt, zu unterscheiden.

Arundel (2001) zeigt, dass F\&E-treibende Unternehmen aller Größenklassen zwar die Geheimhaltung wichtiger ansehen als Patente. Die Wahrscheinlichkeit, dass ein Unternehmen mit Produktinnovationen die Geheimhaltung effektiver bewertet als den Patentschutz, steigt jedoch mit sinkender Unternehmensgröße. Nach Klose / Zimmermann (2008) wächst die eingeschätzte Effektivität von formellen und strategischen Aneignungsmechanismen mit der Unternehmensgröße, wobei jedoch das relative Verhältnis zwischen beiden über alle Größenklassen ungefähr gleich bleibt. Leiponen / Byma (2009) weisen ebenfalls nach, dass kleinere Unternehmen bei der Wahl ihrer Aneignungsstrategie anstatt den Patentschutz eher die Geheimhaltung und den zeitlichen Vorsprung als wichtig ansehen. Kitching / Blackburn (2003) widmen sich in diesem Zusammenhang stärker der Bedeutung für den Innovationserfolg von KMU. In Interviews zur Aneignung von Innovationserträgen befragte EigentümerUnternehmer gaben an, neben der Geheimhaltung hierzu vor allem auch auf andere strategische Aneignungsmechanismen, wie z.B. die langfristige Bindung qualifizierten Personals, zu bauen. Solche, in die allgemeinen Arbeitsroutinen eingebetteten Mechanismen, würden nicht nur zum Schutz von Wissen, sondern auch zur konsequenten Kommerzialisierung von Innovationen genutzt werden. IPRs erschienen hingegen vielen befragten Unternehmern als weitgehend irrelevant für die eigene Innovationstätigkeit. Diese Ergebnisse lassen somit erste Zweifel an der Annahme, das „appropriability“-Problem in KMU sei in erster Linie mit Hilfe einer intensiveren IPR-Nutzung zu lösen, aufkommen

Bezüglich weiterer allgemeiner Studien vgl. z.B. Harabi (1995); Hanel (2008) und Laursen / Salter (2005). 


\section{Nutzung von Aneignungsmechanismen: grundsätzliche Einflussfaktoren}

\section{Datenbasis und deskriptiver Befund am Beispiel Deutschlands}

Die dritte gemeinschaftliche Innovationserhebung der Europäischen Union (CIS 3) bietet für eine Auswahl von formellen und strategischen Aneignungsmechanismen repräsentative Daten für eine Reihe von EWR-Ländern. ${ }^{4}$ Hierbei lag die Frage zu Grunde, ob in den Jahren 1998 bis 2000 von den einzelnen Mechanismen zum Zwecke des Innovationsschutzes mindestens einmal Gebrauch gemacht wurde. Als Grundlage für CIS 3 diente das Oslo-Handbuch (2. Aufl. 1997), das sowohl Leitlinien zur Methodik als auch Definitionen der wichtigsten Innovationskonzepte beinhaltet und somit eine europaweite Vergleichbarkeit der Daten ermöglicht. Auf nationaler Ebene lag die Zuständigkeit zur Durchführung von CIS 3 in der Regel bei den statistischen Ämtern oder einem Ministerium. Das Statistische Amt der Europäischen Gemeinschaft (Eurostat) war koordinierend tätig. In allen Ländern dienten Unternehmen mit mindestens 10 Beschäftigten aus einem breiten Erfassungsbereich von Wirtschaftszweigen (NACE) als statistische Einheit. Mit Ausnahme einer Vollerhebung in Island wurden die Innovationsdaten in allen Fällen mit Hilfe einer geschichteten Zufallsstichprobe erhoben. Diese Werte wurden im Anschluss entsprechend der nationalen Grundgesamtheit hochgerechnet (Larsson 2004, 7; Europäische Kommission, 284 ff.).

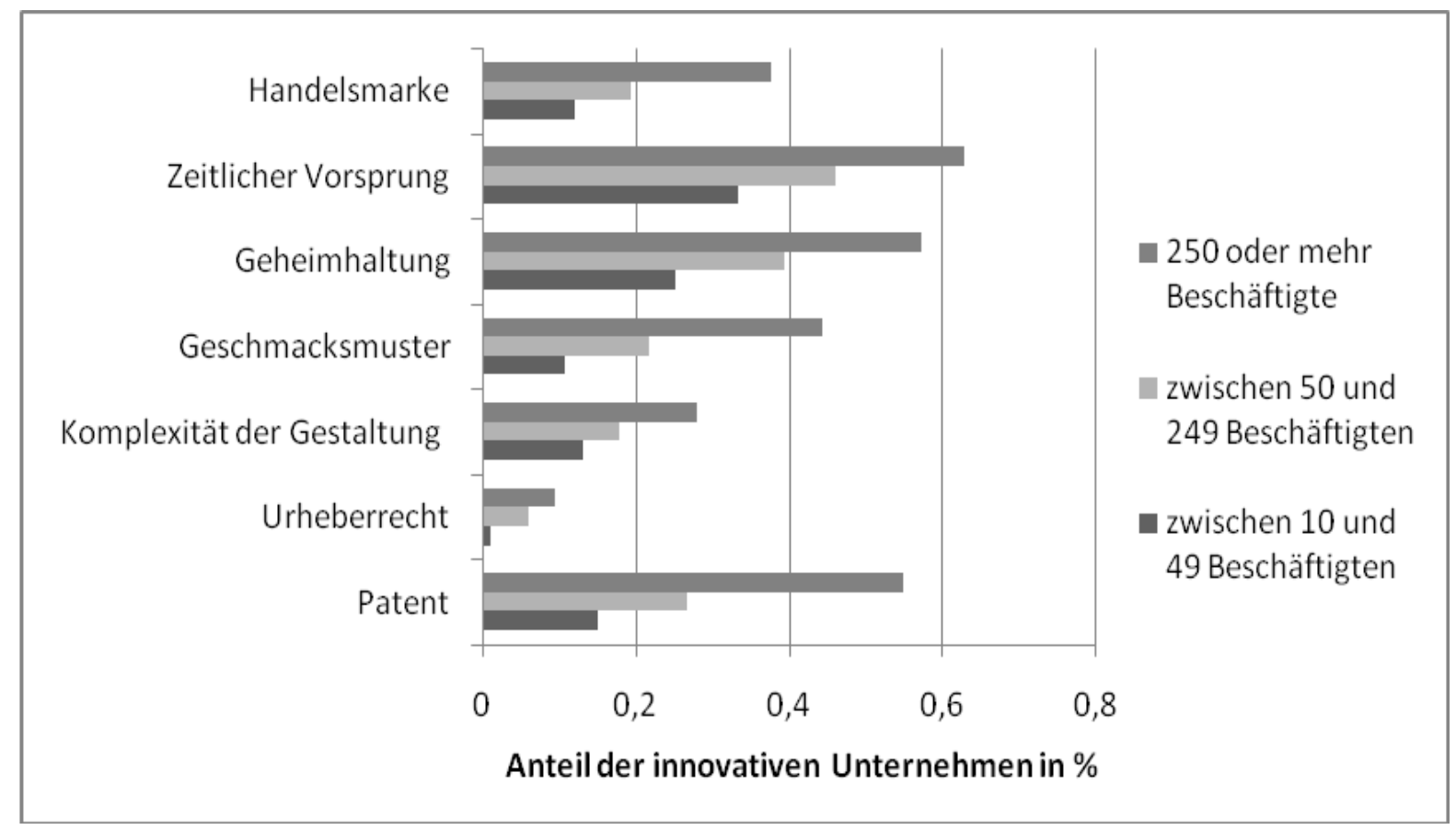

Quelle: Eurostat, CIS 3, Deutschland.

Abbildung 2: Nutzungshäufigkeit von Aneignungsmechanismen in innovativen Unternehmen des deutschen Verarbeitenden Gewerbes

\footnotetext{
4 Hier: Österreich, Belgien, Deutschland, Dänemark, Finnland, Frankreich, Griechenland, Island, Italien, Luxemburg, Niederlande, Norwegen, Portugal, Schweden, Spanien, Vereinigtes Königreich
} 
Die im Folgenden verwendeten CIS 3-Daten liegen in aggregierter Form vor (Anteil der Unternehmen in \%), wobei zwischen der Unternehmensgröße, dem Wirtschaftssektor und dem Innovationstyp differenziert werden kann. Neben einer deskriptiven Auswertung für einzelne Länder erlaubt diese Form der Datenaufbereitung statistische Analysen zur Überprüfung von europaweiten Zusammenhängen im Innovationsverhalten (Jaumotte / Pain 2005).

Tabelle 1: Relative Nutzungsanteile formeller und strategischer Aneignungsmechanismen im deutschen Verarbeitenden Gewerbe

\begin{tabular}{lccccc}
\hline Schutzmechanismus & Kleinunternehmen & & Mittelunternehmen & \multicolumn{2}{c}{ Großunternehmen } \\
\hline Handelsmarke & 10,9 & $<$ & 11,0 & $<$ & 12,8 \\
\hline Geschmacksmuster & 9,8 & $<$ & 12,3 & $<$ & 15,1 \\
\hline Urheberrecht & 0,9 & $<$ & 3,4 & $>$ & 3,2 \\
\hline Patent & 13,6 & $<$ & 15,1 & $<$ & 18,6 \\
\hline Zeitlicher Vorsprung & 30,3 & $>$ & 26,0 & $>$ & 21,4 \\
\hline Geheimhaltung & 22,7 & $>$ & 22,3 & $>$ & 19,4 \\
\hline $\begin{array}{l}\text { Komplexität der } \\
\text { Gestaltung }\end{array}$ & 11,8 & $>$ & 10,0 & $>$ & 9,5 \\
\hline & $100 \%$ & & $100 \%$ & & $100 \%$ \\
\hline
\end{tabular}

Quelle: Eurostat, CIS 3, Deutschland.

Den deutschen Beitrag für CIS 3 lieferte die jährliche Innovationserhebung des Zentrums für Europäische Wirtschaftsforschung (ZEW). Entsprechend des konzeptionellen Ansatzes wurden hierbei insgesamt 71581 bzw. 61 \% der befragten Unternehmen als innovativ klassifiziert (Europäische Kommission, 102). Am Beispiel des deutschen Verarbeitenden Gewerbes zeigt Abbildung 2, dass der Anteil innovativer Unternehmen, die formelle bzw. strategische Aneignungsmechanismen nutzten, mit der Unternehmensgröße steigt. Kleinere Unternehmen nutzten demnach offenbar nicht nur IPRs, sondern auch strategische Aneignungsmechanismen weniger häufig als größere Unternehmen. Dies scheint der Wahrnehmung zu widersprechen, dass KMU stärker auf strategische als auf formelle Mechanismen vertrauen. Eine relative Interpretation der Ergebnisse bestätigt jedoch die Erwartung einer mit sinkender Unternehmensgröße zunehmenden Bedeutung von strategischen Aneignungsmechanismen (vgl. Tabelle 1). Diese Beobachtungen führen daher zu zwei Hypothesen:

$\mathrm{H}$ 1: Der absolute Nutzungsanteil formeller und strategischer Aneignungsmechanismen wächst mit steigender Unternehmensgröße.

H 2: Mit sinkender Unternehmensgröße wächst die relative Bedeutung strategischer Aneignungsmechanismen. 


\section{Statistische Überprüfung durch länderübergreifenden Vergleich}

a) Nutzungshäufigkeit formeller und strategischer Aneignungsmechanismen

Die auf Basis der deskriptiven Auswertung gebildeten Hypothesen werden im Folgenden mit Hilfe der Varianzanalyse (ANOVA) überprüft. Die Varianzanalyse wurde aufgrund des Skalenniveaus der vorliegenden Daten als multivariates Verfahren gewählt, was in Tabelle 2 durch einen Überblick zur Variablenstruktur zum Ausdruck kommt.

Tabelle 2: Variablenstruktur der Varianzanalyse zur Überprüfung von grundsätzlichen

Einflussfaktoren auf die Nutzungshäufigkeit von Aneignungsmechanismen

\begin{tabular}{|c|c|c|c|}
\hline Variablentyp & Bezeichnung & Skalenniveau & Faktorstufen \\
\hline abhängig & $\begin{array}{l}\text { Anteil der Unternehmen } \\
\text { (in \%) }\end{array}$ & metrisch & \\
\hline unabhängig & $\begin{array}{l}\text { Unternehmensgröße } \\
\text { (Beschäftigte) }\end{array}$ & nominal & $\begin{array}{l}1=10 \text { bis } 49 \\
2=50 \text { bis } 249 \\
3=250 \text { oder mehr }\end{array}$ \\
\hline unabhängig & Sektor & nominal & $\begin{array}{l}1 \text { = Verarbeitendes Gewerbe } \\
2 \text { = Dienstleistungssektor }\end{array}$ \\
\hline unabhängig & Innovationstyp & nominal & $\begin{array}{l}1=\text { nur Prozessinnovation } \\
2=\text { nur Produktinnovation } \\
3=\text { beides durchgeführt }\end{array}$ \\
\hline
\end{tabular}

In ausführlicher Form erfolgt an dieser Stelle eine Auswertung zum Aneignungsmechanismus „Patent“ und der „Geheimhaltung“ als dessen strategischem Pendant. Konzipiert als länderübergreifender Vergleich auf Grundlage der europaweiten CIS 3-Daten testet ein dreifaktorielles Varianzanalysemodell die Effekte der Unternehmensgröße, des Sektors und des Innovationstyps auf die Höhe des Anteils der Unternehmen (in \%), die im Befragungszeitraum von diesen Mechanismen Gebrauch gemacht haben. ${ }^{5}$

Die Mittelwerte der einzelnen Faktorstufen (vgl. Tabelle 3) lassen vermuten, dass der Anteil der Unternehmen mit Patentnutzung mit wachsender Unternehmensgröße steigt und im Verarbeitenden Gewerbe deutlicher höher ausfällt als im Dienstleistungssektor. Letzteres dürfte darauf zurückzuführen sein, dass Innovationen im Dienstleistungssektor oftmals nichttechnischer Natur sind und somit den Patentkriterien nicht genügen (Klose / Zimmermann 2008, 5). Des Weiteren nutzen Prozessinnovatoren offenbar deutlich seltener Patente zum Innovationsschutz als Produktinnovatoren. Dies dürfte dadurch zu erklären sein, dass sich Pro-

5 Untersucht werden Quadratwurzel-transfomierte Werte, da nur für diese die Normalverteilungsannahme nicht verworfen werden konnte. Die Varianzhomogenität wurde mit Hilfe der Levene-Teststatistik überprüft. 
zessinnovationen aufgrund ihrer geringeren unternehmensexternen Nachvollziehbarkeit durch strategische Mechanismen effektiver schützen lassen als durch IPRs (Teece 2002, 19).

\section{Tabelle 3: Mittelwerte der Faktorstufen über alle Beobachtungen (Patentnutzung)}

\begin{tabular}{lll}
\hline \multicolumn{1}{c}{ Unternehmensgröße } & \multicolumn{1}{c}{ Sektor } & \multicolumn{1}{c}{ Innovationstyp } \\
\hline 10 bis 49 Beschäftigte $=0,102$ & Verarbeitendes Gewerbe $=0,231$ & Prozessinnovation $=0,087$ \\
50 bis 249 Beschäftigte $=0,174$ & Dienstleistungssektor $=0,140$ & Produktinnovation $=0,222$ \\
250 oder mehr $=0,285$ & & beides durchgeführt $=0,222$ \\
\hline
\end{tabular}

Lesebeispiel: Von den innovativen Unternehmen der Größenklasse 10 bis 49 Beschäftigte nutzten 10,2 \% in den Jahren 1998-2000 mindestens einmal das Patent zum Schutz ihrer Innovationen.

Die Ergebnisse der Varianzanalyse zeigen, dass die Haupteffekte der Faktoren Unternehmensgröße $\left(F_{2,200}=36.69, \mathrm{p}<0.001\right)$, Sektor $\left(F_{1,200}=25.94, \mathrm{p}<0.001\right)$ und Innovationstyp $\left(F_{2,200}=38.10, \mathrm{p}<0.001\right)$ hochsignifikant sind. Des Weiteren bestehen signifikante Wechselwirkungen zwischen den Faktoren Unternehmensgröße und Sektor $\left(F_{2,200}=5.53, \mathrm{p}<0.01\right)$ sowie zwischen den Faktoren Sektor und Innovationstyp ( $\left.F_{2,200}=3.47, \mathrm{p}<0.05\right)$. Im Rahmen der anschließenden Analyse ergibt der paarweise Vergleich der Gruppenmittelwerte mit Hilfe des Tukey HSD-Tests für den Faktor Unternehmensgröße, dass sich alle Größengruppen signifikant zum 5 \%-Niveau voneinander unterscheiden, wobei vor allem die Mittelwertdifferenz zwischen der kleinsten und der größten Unternehmensgruppe auffällig ist (vgl. Tabelle 3). Für die Faktoren Sektor und Innovationstyp bestätigt sich zum 5\%-Niveau, dass im Verarbeitenden Gewerbe häufiger Patente genutzt werden als im Dienstleistungssektor und dass Unternehmen mit Prozessinnovationen Patente deutlich seltener nutzen.

Die Berechnung der Stärke der Haupteffekte („Omega Quadrat“6) ergibt für die Unternehmensgröße einen großen Effekt $\left(\omega^{2}=0.180\right)$, für den Sektor einen mittleren Effekt $\left(\omega^{2}=0.063\right)$ und für den Innovationstyp ebenfalls einen großen Effekt $\left(\omega^{2}=0.187\right)$. Eine grafische Interpretation zeigt im Falle der Interaktion zwischen den Faktoren Unternehmensgröße und Sektor eine ordinale Wechselwirkung (vgl. Abb. 3). Der Unternehmensgrößeneffekt der Patentnutzung ist demnach zwar in beiden Sektoren deutlich zu beobachten, er fällt im Verarbeitenden Gewerbe jedoch stärker aus. Die grafische Interpretation der Wechselwirkung zwischen den Faktoren Innovationstyp und Sektor legt nahe, dass im Verarbeitenden Gewerbe bei alleiniger Durchführung von Produktinnovationen der Anteil der Unternehmen mit Patentnutzung im Vergleich zum Dienstleistungssektor überproportional ansteigt. Bei beiden Wechselwirkungen handelt es sich um kleine Effekte $\left(\omega^{2}=0.023\right.$ und $\left.\omega^{2}=0.012\right)$.

\footnotetext{
6 Nach Cohen (1988) sind im Rahmen der Varianzanalyse folgende Richtlinien für die Effektstärke $\omega^{2} \mathrm{zu}$ beachten: $0,01=$ klein; $0,06=$ mittel; $0,14=$ groß .
} 


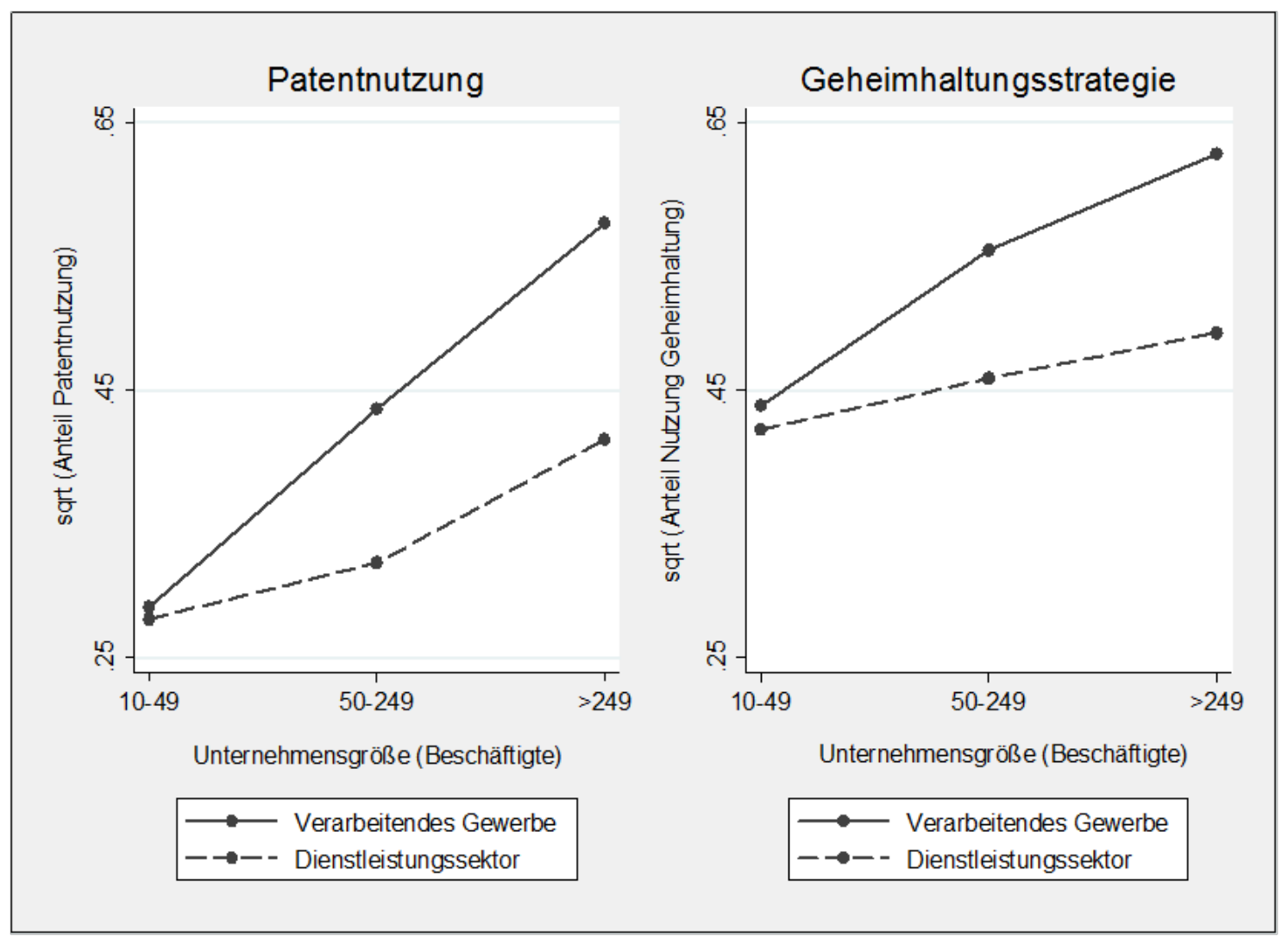

Abbildung 3: Wechselwirkungen zwischen Unternehmensgröße und Sektor

Im Falle der Geheimhaltungsstrategie lassen die Mittelwerte der Faktorstufen vermuten, dass der Nutzungsanteil mit der Unternehmensgröße wächst und im Verarbeitenden Gewerbe höher ausfällt als im Dienstleistungssektor (vgl. Tabelle 4). Produktinnovatoren nutzen zudem die Geheimhaltung offenbar häufiger als Prozessinnovatoren. Erwartungsgemäß ist zudem der Anteil der Prozessinnovatoren, welche auf die Geheimhaltung setzen, deutlich höher als im Falle der Patentnutzung.

Tabelle 4: Mittelwerte der Faktorstufen über alle Beobachtungen (Geheimhaltung)

\begin{tabular}{lll}
\hline \multicolumn{1}{c}{ Unternehmensgröße } & \multicolumn{1}{c}{ Sektor } & \multicolumn{1}{c}{ Innovationstyp } \\
\hline 10 bis 49 Beschäftigte $=0,209$ & Verarbeitendes Gewerbe $=0,320$ & Prozessinnovation $=0,167$ \\
50 bis 249 Beschäftigte $=0,290$ & Dienstleistungssektor $=0,248$ & Produktinnovation $=0,271$ \\
250 oder mehr $=0,357$ & & beides durchgeführt $=0,375$ \\
\hline
\end{tabular}

Lesebeispiel: Von den innovativen Unternehmen der Größenklasse 10 bis 49 Beschäftigte nutzten 20,9 \% in den Jahren 1998-2000 mindestens einmal die Geheimhaltungsstrategie zum Schutz ihrer Innovationen.

Die Varianzanalyse ergibt in diesem Fall eine hohe Signifikanz der Faktoren Unternehmensgröße $\left(F_{2,200}=11.13, \mathrm{p}<0.001\right)$, Sektor $\left(F_{1,200}=20.58, \mathrm{p}<0.001\right)$ und Innovationstyp $\left(F_{2,200}=41.42, \mathrm{p}<0.001\right)$. Des Weiteren besteht eine schwach-signifikante Wechselwirkung zwischen den Faktoren Unternehmensgröße und Sektor $\left(F_{2,200}=3.00, \mathrm{p}<0.1\right)$. Der Tukey 
HSD-Test ergibt, dass sich innovative Kleinunternehmen bei der Nutzung der Geheimhaltungsstrategie jeweils von Mittel- und Großunternehmen signifikant zum 5\%-Niveau unterscheiden. Anders als Tabelle 4 zunächst vermuten lässt, kann zwischen den beiden anderen Größenklassen kein signifikanter Unterschied festgestellt werden. Für die Faktoren Sektor und Innovationstyp bestätigt sich zum 5\%-Niveau die bereits geäußerte Vermutung, dass im Verarbeitenden Gewerbe häufiger die Geheimhaltungsstrategie genutzt wird als im Dienstleistungssektor und dass sich alle Innovationstypen voneinander unterscheiden. Die Effektstärkenberechnung ergibt für die Unternehmensgröße und den Sektor einen mittleren Effekt $\left(\omega^{2}=0.061\right.$ bzw. $\left.\omega^{2}=0.060\right)$ sowie für den Innovationstyp einen großen Effekt $\left(\omega^{2}=0.243\right)$. Eine grafische Interpretation der Wechselwirkung zeigt, dass auch im Falle der Geheimhaltungsstrategie der Größeneffekt im Verarbeitenden Gewerbe stärker ausfällt als im Dienstleistungssektor. Insgesamt ist er jedoch schwächer ausgeprägt als im Falle der Patentnutzung (vgl. Abb. 3). Hierbei handelt es sich um einen kleinen Effekt $\left(\omega^{2}=0.012\right)$.

Eine Varianzanalyse bezüglich der übrigen formellen und strategischen Aneignungsmechanismen brachte als wesentliches Ergebnis ebenfalls eine Signifikanz des Unternehmensgrößeneffekts bei hoher bzw. mittlerer Effektstärke. Die weitere Analyse ergab, dass dies vor allem auf einen geringeren Nutzungsanteil im Kleinunternehmenssektor zurückzuführen ist.

b) Zur relativen Bedeutung strategischer Aneignungsmechanismen

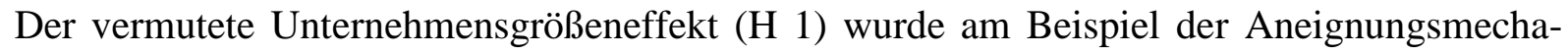
nismen „Patent“ und „Geheimhaltung“ bestätigt. Mit steigender Unternehmensgröße wächst folglich der Anteil der Unternehmen, die formelle bzw. strategische Aneignungsmechanismen nutzen. Im Folgenden wird die Hypothese zur relativen Nutzungshäufigkeit überprüft $(\mathrm{H} 2)$. Hierzu wurden die Daten bzgl. der strategischen Aneignungsmechanismen in einem durchschnittlichen Nutzungsanteil zusammengefasst und zu einem entsprechenden durchschnittlichen IPR-Nutzungsanteil ins Verhältnis gesetzt. Wiederum mit Hilfe der Varianzanalyse wird die relative Bedeutung strategischer Aneignungsmechanismen in Abhängigkeit von der Unternehmensgröße und vom Sektor getestet. ${ }^{7}$ Nur der Haupteffekt des Faktors Unternehmensgröße fällt hierbei signifikant aus $\left(F_{2,74}=4.52, \mathrm{p}<0.05\right)$, was einem mittleren Effekt entspricht $\left(\omega^{2}=0.081\right)$. Der Tukey HSD-Test ergibt, dass dieser Effekt auf einen zum 5 \%-Niveau signifikanten Unterschied zwischen innovativen Klein- und Großunternehmen zurückzuführen ist. Somit bestätigt sich das Bild eines Box-Plot-Vergleichs, nach welchem bereits vermutet werden konnte, dass gerade in Kleinunternehmen strategische Aneignungsmechanismen besonders ins Gewicht fallen (vgl. Abb. 4).

7 Der durchschnittliche IPR-Nutzungsanteil basiert auf Daten zu den Schutzrechten „Patent“, „Geschmacksmuster“ und „Handelsmarke“. Untersucht werden logarithmierte Werte, da nur für diese die Normalverteilungsannahme nicht verworfen werden konnte. Die Varianzhomogenität wurde mit der LeveneTeststatistik überprüft. 


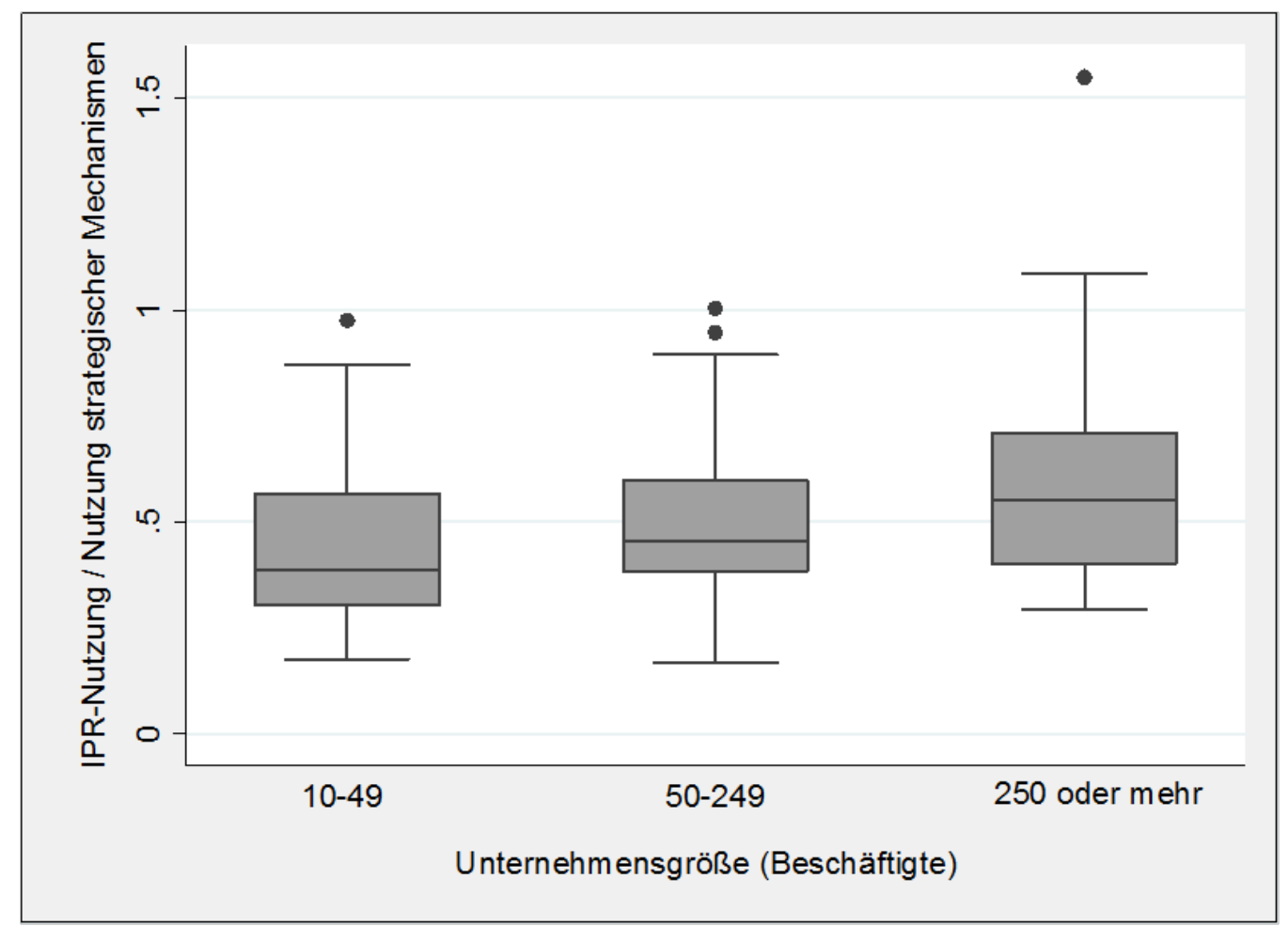

Abbildung 4: Relative Bedeutung strategischer Aneignungsmechanismen in Abhängigkeit von der Unternehmensgröße

\section{Fazit und Diskussion der Ergebnisse}

Der vorliegende Beitrag wirft die Frage auf, warum in KMU intellektuelle Eigentumsrechte (IPRs) weniger genutzt werden als in Großunternehmen. Es wurde dargelegt, dass diesbezüglich insbesondere eine Untersuchung des sogenannten „appropriability“-Problems von Interesse ist. Die Sichtung der theoretischen und empirischen Literatur brachte als wesentliches Ergebnis, dass die KMU-spezifische Aneignung von Innovationserträgen bisher nicht systematisch untersucht worden ist, obwohl in kleineren Unternehmen in diesem Zusammenhang Besonderheiten zu erwarten sind. Eine Einschätzung verschiedener Aneignungsmechanismen hinsichtlich ihrer Nutzungsrelevanz und Effektivität (Imitationsschutz) sowie der damit verbundene Beitrag zum Innovationserfolg kann daher die Bedeutung von IPRs für innovative KMU differenzierter beleuchten, als es bislang der Fall gewesen ist. Angesichts breiter Unterstützungsmaßnahmen zur Förderung einer intensiveren IPR-Nutzung in KMU sind mit einer solchen Untersuchung wichtige wirtschaftspolitische Implikationen verbunden.

Vor diesem Hintergrund wurden grundlegende Einflussfaktoren auf die Nutzungshäufigkeit von Aneignungsmechanismen untersucht. Am Beispiel der Patentnutzung und der Geheimhaltungsstrategie zeigte sich, dass der Anteil der innovativen Unternehmen, die formelle bzw. strategische Mechanismen nutzen, mit der Unternehmensgröße steigt, wobei dieser Effekt im Verarbeitenden Gewerbe stärker ausgeprägt ist als im Dienstleistungssektor. Eine relative Interpretation der Nutzungsanteile führte zu dem Ergebnis, dass strategische Aneig- 
nungsmechanismen jedoch mit sinkender Unternehmensgröße an Bedeutung gewinnen. Hieraus lassen sich zunächst zwei Schlussfolgerungen ableiten.

Erstens bleibt festzuhalten, dass nicht nur die Nutzung von IRRs, sondern auch die Nutzung von strategischen Aneignungsmechanismen mit der Unternehmensgröße wächst. Es wurde aber deutlich, dass hierbei stets zwischen absoluten und relativen Effekten zu trennen ist. Dies ergänzt den Befund von Arundel (2001), der die Notwendigkeit einer relativen Interpretation am Beispiel der eingeschätzten Effektivität von Aneignungsmechanismen herausgearbeitet hat. Zweitens ist der signifikante Unterschied zwischen Klein- und Großunternehmen auffällig. Demnach ist der Anteil der innovativen Kleinunternehmen, die formelle bzw. strategische Aneignungsmechanismen nutzen, deutlich geringer als der Anteil entsprechender Großunternehmen. Bei der Interpretation dieses Ergebnisses ist zu berücksichtigen, dass das verwendete Maß zur Messung der Nutzungshäufigkeit bereits für wichtige Wirkungen der Unternehmensgröße kontrolliert. Denn zum einen wurden die Unternehmen gefragt, ob sie im Befragungszeitraum die jeweiligen Aneignungsmechanismen mindestens einmal zum Schutz ihrer Innovationen nutzten. Zum anderen beziehen sich die Auswertungsergebnisse auf den Anteil der innovativen Unternehmen in der jeweiligen Größenklasse, die von den verschiedenen Aneignungsmechanismen Gebrauch gemacht haben. Auch vor dem Hintergrund des Literaturbefunds, dass zur Aneignung von Innovationserträgen und damit einhergehend zur Rolle von IPRs in innovativen Kleinunternehmen wenig bekannt ist, stellt sich daher für weiterführende Forschungsanstrengungen die Frage, warum diese sich derart unterschiedlich verhalten. Verschiedene Gründe könnten hierfür verantwortlich sein:

Zunächst sind größenbedingte Benachteiligungen im IPR-System nicht per se von der Hand zu weisen. Großunternehmen können die verschiedenen, im Rahmen der Nutzung von formellen Schutzrechten anfallenden Kosten besser schultern als kleinere Unternehmen. Trotz der obigen Einschränkungen bleibt auch festzuhalten, dass kleinere Unternehmen seltener verschiedene Innovationsprojekte parallel durchführen, wodurch insgesamt sowohl die Nutzung formeller als auch strategischer Aneignungsmechanismen weniger dringlich sein könnte (Aschhoff et al. 2007, 116). Es ist jedoch zu klären, ob wirklich nur die Rolle des Unternehmensgrößeneffekts ausschlaggebend ist. Die obige Auswertung deutet bereits darauf hin, dass dies nicht unbedingt der Fall ist. Viele der von Kleinunternehmen generierten Innovationen dürften z.B. auch überhaupt nicht in den Schutzbereich von IPRs fallen, da sie oftmals inkrementeller Natur sind (Abel 2006, 68 ff.). In diesem Fall würden andere (verfügbare) Aneignungsmechanismen zwangsläufig eine größere Rolle spielen. Zudem bleibt auch zu klären, ob und - falls ja - warum IPRs für kleinere Unternehmen weniger effektiv wirken. Denn andere Aneignungsmechanismen könnten womöglich in Kleinunternehmen viel besser funktionieren. Die Rolle des „tacit knowledge“ ist in diesem Zusammenhang noch weitgehend unklar, obwohl gerade das unternehmensspezifische Erfahrungswissen für die dynamischen und wenig formalisierten Innovationsprozesse in kleineren Unternehmen von großer Bedeutung ist (Nooteboom 1994, 334 ff.; Lahner 2004, 285). Im Rahmen des kleinbetrieblichen Innovationsgeschehens dürfen auch die häufig zu beobachtende Konzentration auf Nischenmärkte und die hohe Bedeutung der Kundennähe, die sich in innovativen Problemlö- 
sungen für einen sehr beschränkten Anwenderkreis niederschlagen kann, nicht außer Acht gelassen werden (Lahner 2008, 54 ff.). Denn wie gezeigt bestimmt sich die Aneignung von Innovationserträgen gerade auch durch die Replizierbarkeit einer Innovation. Die Möglichkeit zur Imitation durch die Konkurrenz sinkt folglich mit dem Anstieg der Spezifität des zu schützenden Innovationswissens. Zugleich sollte die Relevanz einzelner Aneignungsmechanismen für den Innovationserfolg berücksichtigt werden. Der notwendige Ausgleich zwischen Wissensaustausch und Wissensschutz dürfte im Falle des Innovationserfolgs von kleineren Unternehmen Besonderheiten aufweisen. Ein hoher Grad an „tacit knowledge“ wäre dann z.B. differenziert zu sehen, da dieser neben einem höheren Imitationsschutz in der Regel auch mit einer verminderten Absorptionsfähigkeit neuer, innovationsrelevanter Informationen einhergeht (Nooteboom 1994, 334). Die häufig zu beobachtende Strategie innovativer KMU, diese Schwäche durch informelle externe Wissensflüsse (z.B. Netzwerke mit Zulieferern und Kunden) zu kompensieren, müsste dann auch unter diesem Gesichtspunkt eingeordnet werden. Im Sinne von Teece (1986) ist hiermit die Bedeutung von komplementären Gütern für den Innovationserfolg von KMU angesprochen (Baldwin / Gellatly 2003, 277 ff.).

Abschließend bleibt somit festzuhalten, dass - beim Versuch die Bedeutung von IPRs für innovative KMU zu klären - eine einseitige Konzentration auf mögliche Nachteile der kleineren Unternehmensgröße zu kurz greift. Dies dennoch zu versuchen erinnert an die Diskussion des Zusammenhangs zwischen Unternehmensgröße und Innovation im Sinne der NeoSchumpeter-Hypothese. Diese konnte empirisch nicht bestätigt werden und hat inzwischen einem neuen Innovationsverständnis Platz gemacht, wonach die Unternehmen der einzelnen Größenklassen auf Grundlage ihrer jeweiligen Stärken und Schwächen ergänzende Innovationsbeiträge leisten (Lahner 2004, 101 ff.). Eine zukünftige Einschätzung der Bedeutung von IPRs für innovative KMU sollte folglich auch die Heterogenität im Innovationsverhalten stärker in den Mittelpunkt rücken. Oder anders ausgedrückt: Nur weil in kleineren Unternehmen der Schutz von Innovationen anders funktioniert, muss dies nicht automatisch schlecht sein.

\section{Literatur}

Abel, R. (2006): Innovationen in Kleinunternehmen: Wahrnehmung, Wirklichkeit und Wege. In: Abel, R. / Bass, H. H. / Ernst-Siebert, R. (Hrsg.): Kleine und mittelgroße Unternehmen im globalen Innovationswettbewerb. München, Mering: Rainer Hampp, 63-87.

Arrow, K. J. (1962): Economic Welfare and the Allocation of Resources for Invention. In: Nelson, R. R. (Hrsg.): The Rate and Direction of Inventive Activity. Princeton, NJ: Princeton University Press, 609-625.

Arundel, A. (2001): The relative effectiveness of patents and secrecy for appropriation. In: Research Policy, 30 (4), 611-624.

Aschhoff, B. / Blind, K. / Ebersberger, B. / Fraaß, B. / Rammer, C. / Schmidt, T. (2007): Schwerpunktbericht zur Innovationserhebung 2005. Bericht an das Bundesministerium für Bildung und Forschung (BMBF). ZEW-Dokumentation Nr. 07-03. 
Baldwin, J. / Gellatly, G. (2003): Innovation Strategies and Performance in Small Firms. Cheltenham, UK: Edward Elgar.

Blackburn, R. A. (2003): Small firms, innovation and intellectual property management: the context and a research agenda. In: Blackburn, R. A. (Hrsg.): Intellectual Property and Innovation Management in Small Firms. London, New York: Routledge, 4-15.

Blind, K. / Cuntz, A. / Köhler, F. / Radauer, A. (2009): Die volkswirtschaftliche Bedeutung geistigen Eigentums und dessen Schutzes mit Fokus auf den Mittelstand. Eine Studie im Auftrag des Bundesministeriums für Wirtschaft und Technologie. Berlin.

Bosworth, D. / Webster, E. (2006): An economic perspective. In: Bosworth, D. / Webster, E. (Hrsg.): The Management of Intellectual Property. Cheltenham: Edward Elgar, 85-107.

Burr, W. / Stephan, M. / Soppe, B. / Weisheit, S. (2007): Patentmanagement - Strategischer Einsatz und ökonomische Bewertung von technologischen Schutzrechten. Stuttgart: Schäffer-Poeschel.

Cohen, J. (1988): Statistical power analysis for the behavioral sciences. Hillsdale, NJ: Erlbaum.

Cohen, W. M. / Nelson, R. R. / Walsh, J. P. (2000): Protecting Their Intellectual Assets: Appropriability Conditions and Why U.S. Manufacturing Firms Patent (or Not). In: NBER Working Papers Series, 7552.

David, P. A. (2004): Can "Open Science" be Protected from the Evolving Regime of IPR Protections? In: Journal of Institutional and Theoretical Economics, 160, 1-26.

Dosi, G. / Malerba, F. / Ramello, G. B. / Silva, F. (2006a): Information, appropriability, and the generation of innovative knowledge four decades after Arrow and Nelson: an introduction. In: Industrial and Corporate Change, 15 (6), 891-901.

Dosi, G. / Marengo, L. / Pasquali, C. (2006b): How much should society fuel the greed of innovators? On the relations between appropriability, opportunities and rates of innovation. In: Research Policy, 35 (8), 1110-1121.

Europäische Kommission, (2004): Innovation in Europe: Results for the EU, Iceland and Norway. Luxemburg: Office for Official Publications of the European Communitites.

González-Álvarez, N. / Nieto-Antolín, M. (2007): Appropriability of innovation results: An empirical study in Spanish manufacturing firms. In: Technovation, 27 (5), 280-295.

Greenhalgh, C. / Rogers, M. (2007): The Value of Intellectual Property Rights to Firms and Society. In: Oxford Review of Economic Policy, 23 (4), 541-567.

Hall, M. / Oppenheim, C. / Sheen, M. (2003): Barriers to the use of patent information in SMEs. In: Blackburn, R. A. (Hrsg.): Intellectual Property and Innovation Management in Small Firms. London, New York: Routledge, 144-160.

Hanel, P. (2008): The use of intellectual property rights and innovation by manufacturing firms in Canada. In: Economics of Innovation and New Technology, 17 (3), 285-309.

Harabi, N. (1995): Appropriability of Technical Innovations. An Empirical Analysis. In: Research Policy, 24 (6), 981-992.

Hurmelinna, P. / Kyläheiko, K. / Jauhiainen, T. (2007): The Janus face of the appropriability regime in the protection of innovations: Theoretical re-appraisal and empirical analysis. In: Technovation, 27 (3), 133-144. 
Hurmelinna-Laukkanen, P. / Puumalainen, K. (2007): Nature and dynamics of appropriability: strategies for appropriating returns on innovation. In: R\&D Management, 37 (2), 95-112.

Hurmelinna-Laukkanen, P. / Sainio, L.-M. / Jauhiainen, T. (2008): Appropriability regime for radical and incremental innovations. In: R\&D Management, 38 (3), 278-289.

Hurmelinna-Laukkanen, P. (2009): The availability, strength and efficiency of appropriability mechanisms - protecting investments in knowledge creation. In: International Journal of Technology Management, 45 (3), 282-290.

Iversen, E. (2003): Norwegian Small and Medium-Sized Enterprises and the Intellectual Property Rights System: Exploration and Analysis. Eine Studie im Auftrag der World Intellectual Property Organization (WIPO). Oslo.

Jaumotte, F. / Pain, N. (2005): From Innovation Development to Implementation: Evidence from the Community Innovation Survey. In: OECD Economics Department Working Papers, 458.

Kitching, J. / Blackburn, R. A. (2003): Innovation, intellectual property and informality: evidence from a study of small enterprises and some implications for policy. In: Blackburn, R. A. (Hrsg.): Intellectual Property and Innovation Management in Small Firms. London, New York: Routledge, 16-34.

Klose, J. / Zimmermann, V. (2008): Wie schützen kleine und mittlere Unternehmen ihre Innovationen? Die Bedeutung rechtlicher und strategischer Schutzinstrumente im Vergleich. In: KfW-Research, WirtschaftsObserver online, 39.

Lahner, J. (2004): Innovationsprozesse im Handwerk. Duderstadt: Mecke Druck.

Lahner, J. (2008): Innovative Kleinunternehmen - systematisch unterschätzt oder nur übersehen? In: RegioPol - Zeitschrift für Regionalwirtschaft, 2, 53-61.

Lanjouw, J. O. / Schankerman, M. (2004): Protecting Intellectual Property Rights: Are Small Firms Handicapped? In: The Journal of Law \& Economics, 47 (1), 45-74.

Larsson, A. (2004): Innovationsergebnisse und -hemmnisse. In: Statistik kurz gefasst 1/2004. Eine Schriftenreihe der Europäischen Kommission. Luxemburg.

Laursen, K. / Salter, A. (2005): My Precious. The Role of Appropriability Strategies in Shaping Innovative Performance. In: DRUID Working Paper No. 05-02.

Leiponen, A. / Byma, J. (2009): If you cannot block, you better run: Small firms, cooperative innovation, and appropriation strategies. In: Research Policy, 38 (9), 1478-1488.

Levin, R. C. / Klevorick, A. K. / Nelson, R. R. / Winter, S. G. (1987): Appropriating the Returns from Industrial Research and Development. In: Brookings Papers on Economic Activity, 3, 783-831.

Macdonald, S. (2003): Bearing the Burden: Small Firms and the Patent System. In: Journal of Information, Law and Technology (JILT), 2003 (1), http://www2.warwick.ac.uk/fac/soc/law/elj/jilt/2003_1/macdonald.

Malerba, F. (2002): Sectoral systems of innovation and production. In: Research Policy, 31 (2), 247-264.

Meyer, J.-A. (2001): Innovationsmanagement in kleinen und mittleren Unternehmen, Jahrbuch der KMU- Forschung (hrsg. von Jörn-Axel Meyer). München: Vahlen.

Nelson, R. R. (1959): The simple economics of basic scientific research. In: The Journal of Political Economy, 67 (3), 297-306. 
Nelson, R. R. (2006): Reflections of David Teece's "Profiting from technological innovation...". In: Research Policy, 35 (8), 1107-1109.

Nooteboom, B. (1994): Innovation and Diffusion in Small Firms: Theory and Evidence. In: Small Business Economics, 6 (5), 327-347.

Radauer, A. / Streicher, J. / Ohler, F. (2007): Benchmarking National and Regional Support Services for SMEs in the Field of Intellectual and Industrial Property. Eine Studie im Auftrag der Europäischen Kommission. Wien.

Rothwell, R. (1983): Innovation and firm size: a case for dynamic complementarity; or, is small really so beautiful? In: Journal of General Management, 8(3), 5-25.

Schumpeter, J. A. (1942): Kapitalismus, Sozialismus und Demokratie, Originaltitel: Capitalism, Socialism and Democracy, 8. unveränderte Auflage (2005). Tübingen: Francke.

Scotchmer, S. (2004): Innovation and Incentives. Cambridge: MIT Press.

Teece, D. J. (1986): Profiting from technological innovation: Implications for integration, collaboration, licensing and public policy. In: Research Policy, 1986 (15), 285-305.

Teece, D. J. (2002): Managing Intellectual Capital. Organizational, Strategic, and Policy Dimensions. Oxford: Oxford University Press.

WIPO (2003): Intellectual Property (IP) Rights and Innovation in Small and Medium-sized Enterprises, World Intellectual Property Organization (WIPO). Genf. 


\section{Kapitel III}

To protect or not to protect?

Modes of appropriability in the small enterprise sector 


\section{Introduction}

The varying ability of innovators to protect themselves from imitation and to appropriate an adequate proportion of innovation returns is regarded as an important driver of diversity in innovation activities both at the firm and the sector level (Levin et al., 1985; Pavitt, 1984; Teece, 1986).

Intellectual Property Rights (IPRs), especially patents, play a major role in this discussion. In theory, IPRs are an effective mechanism for resolving the appropriability problem of knowledge. Indeed, the standard justification for granting IPRs is that they induce incentives to produce socially desirable innovations, thereby mitigating the effects of innovation market failure (Granstrand, 1999; Greenhalgh and Rogers, 2007; Guellec and Van Pottelsberghe de la Potterie, 2007). Based on this assumption, the fostering of IPR usage by small and medium enterprises (SMEs) is regarded as an integral part of innovation policy. Despite their widely recognized importance for innovations, smaller firms often refrain from using registered IPRs. One explanation may be that SMEs are disadvantaged by their smaller company size when it comes to the awareness, acquisition and enforcement of IPRs (Cohen et al., 2000; Hall et al., 2003; Lanjouw and Schankerman, 2004; Macdonald, 2004; Rothwell, 1983). Thus, to attenuate these potential impediments to innovation the strengthening of IPR usage by SMEs is regarded as a major task for policy-makers (European Commission, 2006; PRO INNO Europe, 2007; Radauer et al., 2007; WIPO, 2003).

However, according to Jensen and Webster (2006) this must not be the end of the story. They argue that policy makers should first take into account the general appropriability conditions that small firms face before focusing on their ability to utilize IPRs. Similarly, albeit more generally, Scotchmer (2004) points out that it is always better to start from the appropriability problem itself rather than assume at the outset that IPRs are the best solution. Hence, two aspects take on special interest.

Firstly, the prevalence of innovation market failure in sectors or industries has to be assessed. In the present context this depends to a large degree on the inherent replicability of technology and the subsequent ease of imitation by competitors. At one extreme there is market failure because the relevant knowledge base is fully codifiable, leaving the marginal costs of imitation at zero. In this instance one would expect a strong positive link between IPR protection and innovation incentives. At the other extreme the critical knowledge base is highly tacit in nature. Apart from the hiring away of key employees, imitation by competitors may now be impossible. Lack of innovation incentives owing to low appropriability should therefore be less of a problem, since a high degree of knowledge tacitness serves in itself as a strong protection mechanism (Dosi et al., 2006; Hurmelinna-Laukkanen and Puumalainen, 2007; Jensen and Webster, 2006; Teece, 2002, 2003). Indeed, much of the operating knowledge in small firms tends to be tacit. Tacitness therefore works as an effective appropriation method, in particular for small firms (Nooteboom, 1994). Moreover, patents may not be available to a great number of small firms precisely because their tacit knowledge base cannot be reduced to codified information.

Secondly, even in the case of potential market failure a low level of IPR usage does not give rise to public concern when effective remedies are available. A number of studies have 
shown the relative unimportance of IPRs as a means of profiting from innovation. Other appropriation methods such as lead time, secrecy or complementary assets are deemed in most industries to be more effective than patents (e.g. Cohen et al., 2000; Harabi, 1995; König and Licht, 1995; Laursen and Salter, 2005; Levin et al., 1987; Mansfield, 1986). ${ }^{1}$ Some of these studies also discuss the impact of firm size on the perceived effectiveness of IPR protection. Broadly speaking, they come to the conclusion that smaller firms are less likely to make use of IPRs because of cost and complexity issues, and instead probably rely on informal methods such as secrecy (e.g. Arundel, 2001; González-Álvarez and Nieto-Antolín, 2007; Hanel, 2008; Sattler, 2003).

Despite the economic significance of SMEs, however, very few studies explicitly address the general appropriability conditions of small firms and draw conclusions for innovation policy from their findings. Kitching and Blackburn (1998) examined this issue systematically for the first time (see also Kitching and Blackburn, 2003). From a telephone survey of small firms in the UK (four sectors: computer software, design, electronics, mechanical engineering) and subsequent face-to-face interviews, the authors showed that most SME owner-managers preferred informal protection practices (e.g. creating high-trust relations with customers and suppliers, maintaining a lead time advantage over competitors or operating in small niche markets) because they found them more familiar, cheaper, less time-consuming and more effective than IPRs. Furthermore, owner-managers saw these practices as vital to the commercialization of innovations and hence as a key component of their broader competitive strategy. Thus, the majority of small business owners did not consider access to or the use of IPRs as an impediment to the successful appropriation of innovation returns. Indeed, most owner managers were largely indifferent to the IPR system, since they felt that it neither facilitated nor hindered their innovative efforts. On the other hand, the use of IPRs was only reported under extremely selective conditions. SME owner-managers preferred IPRs in situations where the potential benefits were perceived to outweigh any potential acquisition or enforcement costs. Anticipation of a high degree of commercial innovation success, an appraisal of IPRs as more effective than informal methods and the possession of the necessary resources to acquire formal protection constituted the prerequisites here. From their results, Kitching and Blackburn $(1998,2003)$ conclude that policy attempts to remove barriers to IPR usage may have little impact on innovation by SMEs. In their view, instead of focusing on the protection of existing innovations through easier access to IPRs, policy makers should promote the introduction of new innovations in SMEs.

The study by Leiponen and Byma (2009) also has an explicit focus on small firms. According to these authors, the appropriation strategies pursued by small firms differ qualitatively from those of larger firms. In a survey of knowledge-intensive Finnish SMEs in the manufacturing and service industries, it is again shown that a great number of small firms prefer informal protection practices to IPRs. Only highly R\&D-intensive small firms and those that cooperate with universities in R\&D saw patents as the most important protection instrument. Indeed, it becomes evident that innovation-related cooperation activities in general have a major

1 See López (2009) for a comprehensive literature review on this topic. 
impact on the kind of appropriation strategies chosen by SMEs. Furthermore, the majority of small firms did not consider secrecy to be the most important protection mechanism. Instead they tended for the most part to benefit from a speed to market strategy in their efforts to achieve a lead time advantage over competitors. From their results, Leiponen and Byma (2009) argue that small firms may be disadvantaged by their size in the use of IPRs, and suggest a critical re-evaluation of current patent-focused IPR policies. Moreover, they discuss several proposals on how the IPR system might provide more support for SMEs.

Using a large sample size, the aim of our paper is to corroborate existing empirical evidence and to deepen the understanding of appropriation strategies taken by innovative small firms. Several issues deserve further investigation. Firstly, SMEs should not be treated as a single entity. Instead, the strong skewness in firm size distribution towards smaller enterprises requires consideration with regard to the great diversity among small firms. In this way policy-makers will be in a better position to meet the specific needs of certain SMEs (Curran and Blackburn, 2001). Taxonomies of innovation are a common method of accounting for such heterogeneity at the firm or sector level (Evangelista, 2000; Hollenstein, 2003; Jensen et al., 2007; Pavitt, 1984). As de Jong and Marsili (2006) have indicated, the taxonomic approach is particularly beneficial when studying the variability of innovative small firms. Yet, they did not focus on appropriation strategies in their identification and profiling of distinct clusters of small firms. We therefore seek to determine and characterize different modes of appropriability in the small enterprise sector. In this way, we will not have to treat the different innovation protection mechanisms under review as separate choices or even as mutually exclusive, as is the case in most studies on this topic (for an exception, see Amara et al., 2008). An examination of their interplay seems especially promising, since the strength of individual appropriation methods often lies in their combined use (Bosworth and Webster, 2006). As a further advantage we can interpret the use of IPRs by small firms within the context of their overall appropriation strategy. In so doing, policy-makers may arrive at a better understanding of the general importance of patents in the ability of small firms to profit from innovation (Arundel, 2000).

Secondly, as the above discussion suggests, it might be argued that the less frequent SME usage of IPRs not only results from size-related disadvantages inherent in the IPR system but also reflects specific features of innovation protection practices in small firms. In fact, it is worth noting that small firms are not merely a scaled-down version of large firms (Penrose, 1959). Since the former are less likely to introduce $R \& D$-intensive innovations that are fundamentally new, the novelty of their innovations is frequently determined differently from that of large firms. Because of behavioural advantages in terms of flexibility and speed of response, innovation in smaller firms is often associated with a better differentiation of existing products by focusing on superior customer service or by the fast, flexible and incremental adjustment of product quality to customer needs (Appiah-Adu and Singh, 1998; Baldwin and Gellatly, 2003; Mazzarol and Reboud, 2009; Wynarczyk et al., 1993). As a result, for example, in consideration of the framework of Teece (1986), complementary assets such as sales, services or manufacturing capabilities may in many cases be of greater importance in the successful commercialization of small firm innovation than the protection of core technological know-how via IPRs. Thus, to determine whether the lower use of IPRs by SMEs 
might also be related to such factors, this paper aims to interpret the appropriation strategies of innovative small firms in the light of their specific business contexts.

Thirdly, evidence from Kitching and Blackburn (1998, 2003) suggests that small business owners use informal methods not only to protect their innovations but also to commercialize them. Thus, two key conditions regarding the appropriability of innovations were reported. ${ }^{2}$ Preventing imitation through strong protection is one important condition for an effective appropriation strategy. Nevertheless, a second, albeit crucial, condition consists in the degree to which protection methods are likely to increase innovation returns and enable innovators to garner sufficient profit from their innovations (Hurmelinna-Laukkanen, 2009; Jantunen and Hurmelinna-Laukkanen, 2006; Teece, 1986, 2006). Studies of appropriability frequently focus on the first condition, implicitly assuming that the second condition has been met. However, a comparison of different appropriation strategies to assess the relevance of IPRs for innovative small firms also calls for consideration of their effectiveness with regard to company goals. Moreover, in this way more insight can be derived into which kinds of innovation are protected by which types of protection mechanism. A further purpose of our paper is therefore to give some indication of the relationship between firm performance as measured by several innovation effects and distinct modes of appropriability in the small enterprise sector.

The rest of the paper is structured as follows: Section 2 describes the data set and the variables used. In Section 3, factor analysis and cluster analysis are combined to identify distinct modes of appropriability in the small enterprise sector. Assuming that this taxonomy represents different types of appropriation strategy chosen by innovative small firms, Section 4 assesses the predictive validity of the derived cluster solution and describes its characteristics in relevant business dimensions. Section 5 provides some concluding remarks and discusses the implications for policy and research.

\section{Data set and variables}

Our empirical analysis rests on data from the German Innovation Survey ("Mannheim Innovation Panel"), which is conducted annually by the Centre for European Economic Research (ZEW) on behalf of the German Federal Ministry of Education and Research. Based on a broad set of questions covering all fields of innovation, this survey offers detailed information on the innovation activities of firms in the manufacturing and service sectors. To carry out the analysis we used anonymised data from the survey wave of 2005. In that year the survey constituted the German part of the fourth EU-wide Community Innovation Survey (CIS IV) covering the three-year reference period 2002-2004. While the German Innovation Survey corresponds fully with the harmonized CIS questionnaire with regard to content and methodology, it has distinct advantages over the CIS. First of all, data is available on a wider selection of questions, allowing the relationship between the innovation activities of firms, their market environments and their economic performance to be analysed in more depth. In

We apply the conventional definition as proposed, for example, by Malerba (2002, p. 252): “Appropriability of innovations summarizes the possibilities of protecting innovations from imitation and of reaping profits from innovative activities”. 
the present case, for example, we have information not only on the use of IPR protection and other informal methods such as secrecy or lead time advantage as measured on a binary scale (i.e. 'yes' or 'no'), but also on their degree of importance on an ordinal scale. Furthermore, compared with the CIS, the German Innovation Survey has a somewhat broader sector and size coverage. It also includes firms with between 5 and 9 employees and has a larger set of service sectors. Both aspects are especially beneficial to our subject of investigation (see Janz et al., 2001 and Rammer et al., 2009 for a general discussion of the German Innovation Survey).

The population of the 2005 survey wave was drawn as a stratified random sample. The variables used for stratification were industry classification (mainly at the 2-digit sectoral level), firm size (7 to 8 size classes, depending on the industry sector) and region (East or West Germany). The gross sample, corrected for neutral losses (owing to firm closure, mergers etc.), comprised a total of 27,926 firms, 5,476 of which returned a questionnaire. This represents a response rate of $20 \%$. A stratified random sample was drawn from the group of non-responding firms to check for a potential bias resulting from unit non-response. About $19 \%$ of non-respondents were contacted in a telephone survey to answer several core innovation-related questions (Aschhoff et al., 2007).

Since we are interested in the roles of different protection mechanisms in innovative small firms, we restricted our data set in two ways. Firstly, the analysis was confined to the subsample of innovators. A company was classified as innovative if it had introduced product and/or process innovations during the reference period 2002-2004 or if it reported innovation projects not yet completed in this time frame. Secondly, for several reasons, we removed firms with more than 49 employees from the dataset. Given the strong skewness in company size distribution towards smaller firms, it could be argued that the heterogeneity of appropriation strategies - our interest - is likely to be highest among small-sized firms. Furthermore, since our study also aims to inform policy-making more effectively in this field, we decided to focus on one important subgroup of innovative SMEs. Finally, as Greenhalgh and Rogers (2007, p. 563) point out, existing empirical research on the potential disadvantages of smaller firms using IPRs "has generally neglected to include information about the smallest firms (i.e. those with fewer than 50 employees)". Accordingly, our sample contains data on 1,624 innovative small firms which have between 5 and 49 employees.

The primary variables used for the empirical analysis in Section 3 are based on a set of questions in which firms were asked if they had used certain innovation protection mechanisms during the reference period, that is, those of patent, utility model ${ }^{3}$, industrial design, trademark, copyright, secrecy, complexity of design and lead time advantage. The first five of these constitute formal protection methods. They are granted as exclusive rights on intellec-

The utility model was introduced as a "petty patent" in some countries (including Germany) to provide a cheaper but simpler alternative to patent protection. It is deemed particularly suited to the specific needs of SMEs. Utility models are available for less inventive steps (incremental improvements and adaptations of existing products), can be registered more quickly and are less expensive to acquire and maintain than patents. Compared with patents, however, they have a shorter protection term and provide less legal protection (Radauer et al., 2007; WIPO, 2004). 
tual property for a certain period of time. The final three comprise informal efforts by innovators to protect themselves against imitation. They either prevent unintended knowledge spillover to competitors (secrecy), increase the time and cost required for imitation (complexity of design) or lead to benefits gained from the rapid implementation of innovation projects (lead time advantage). Firms were further asked to assess the importance of the mechanisms used. Therefore, the relevance of an innovation protection method is measured on a 4-point Likert scale $(0=$ no utilization, $1=$ low importance, $2=$ moderate importance, $3=$ high importance). ${ }^{4}$ Since not all respondents provided full information on this set of questions, the number of observations available for statistical analysis in Section 3 was reduced to 1,257.

Descriptive statistics on the primary variables are provided in Table 1. As expected, informal protection practices are, on average, more important for small firm innovators than IPRs. Lead time is most frequently considered to be of moderate or high importance, followed by secrecy and the complexity of design. However, for a certain number of respondents IPR protection is also of moderate or high importance (12.5\% in the case of patents). Furthermore, it becomes evident that in each case a large number of innovative small firms refrained from using the corresponding mechanisms.

\section{Table 1}

Descriptive statistics on the importance of innovation protection mechanisms ( $\mathrm{N}=$ number of small firms; \% = share of sample)

\begin{tabular}{lcccccccc}
\hline & \multicolumn{2}{c}{ No utilization } & \multicolumn{2}{c}{$\begin{array}{c}\text { Low } \\
\text { importance }\end{array}$} & \multicolumn{2}{c}{$\begin{array}{c}\text { Moderate } \\
\text { importance }\end{array}$} & \multicolumn{2}{c}{$\begin{array}{c}\text { High } \\
\text { importance }\end{array}$} \\
\cline { 2 - 9 } & $\mathrm{N}$ & $\%$ & $\mathrm{~N}$ & $\%$ & $\mathrm{~N}$ & $\%$ & $\mathrm{~N}$ & $\%$ \\
\hline Patent & 1062 & 84.9 & 33 & 2.6 & 45 & 3.6 & 111 & 8.9 \\
Utility model & 1108 & 88.6 & 39 & 3.1 & 40 & 3.2 & 64 & 5.1 \\
Industrial design & 1222 & 97.7 & 24 & 1.9 & 4 & 0.3 & 1 & 0.1 \\
Trademark & 1069 & 85.5 & 32 & 2.6 & 61 & 4.9 & 89 & 7.1 \\
Copyright & 1176 & 94.0 & 29 & 2.3 & 13 & 1.0 & 33 & 2.6 \\
$\begin{array}{l}\text { Secrecy } \\
\text { Complexity of }\end{array}$ & 832 & 66.5 & 28 & 2.2 & 117 & 9.4 & 274 & 21.9 \\
design & 1016 & 81.2 & 26 & 2.1 & 79 & 6.3 & 130 & 10.4 \\
$\begin{array}{l}\text { Lead time } \\
\text { advantage }\end{array}$ & 799 & 63.9 & 25 & 2.0 & 82 & 6.6 & 345 & 27.6 \\
\hline
\end{tabular}

Note: The total number of observations amounts to only 1,251 companies in each case, since 6 observations were deleted as outliers in the later cluster analysis (see Section 3.2).

Descriptions of the variables used in Section 4 for validating and profiling the derived appropriation modes are shown in Table A1 (see Appendix). In order to assess the predictive validity of our cluster solution, we follow Hair et al. (1998) in selecting variables that are not

4 The full question asked in the German CIS was: Has your company used one (or more) of the following IP protection mechanisms during 2002-2004? If yes: please indicate its importance for innovation protection in your company. 
used to form clusters but are expected to vary strongly across them. In the light of the discussion above, two variables should be key drivers of variability in the appropriation strategies of innovative small firms: the conducting of in-house R\&D on a permanent basis and engagement in innovation-related cooperation activities. Furthermore, a third input-related variable reflecting different levels of innovation intensity (i.e. the total innovation expenditure as a share of turnover in \%) is used for validation, since more expensive innovations should also require stronger protection efforts to recover these investments.

Next, to provide a better understanding of the choice of protection mechanisms made by innovative small firms, we use a set of variables to characterize each appropriation mode in three key dimensions. Firstly, we are able to use 22 industry dummies that primarily refer to the two-digit level of NACE Rev. $1^{5}$ to describe the industry composition of the clusters. Yet, to account for the fact that some industries are more likely to appreciate protection methods than others, we created a variable of industry groups according to their approximate degree of knowledge intensity (four categories: knowledge-intensive manufacturing, other manufacturing, knowledge-intensive services, and other services). For this purpose we used the NACE Rev.1 classification of knowledge-intensive industries in the case of Germany provided by Grupp et al. (2000). Since the degree of R\&D intensity has drawbacks as an indicator of innovation in service sectors, the criteria used by these authors to assess the knowledge intensity of industries are given as above-average shares of engineering and natural science graduates, of $\mathrm{R} \& \mathrm{D}$ personnel and of general graduates (including graduates from non-technical sciences).

Secondly, we examine the possible influence of several output-oriented measures on the choice of appropriation strategy. To begin with, we examine the roles of basic types of innovators. The fact that smaller firms are less likely to carry out several innovation projects at the same time suggests that their use of innovation protection mechanisms tends to be more selective. We consider four innovator groups: product-only innovators, process-only innovators, comprehensive innovators (small firms engaged in product and process innovations) and nascent innovators (small firms that report only innovation projects not yet completed during the reference period). Because process technology cannot easily be observed from the outside and contains a relatively high degree of knowledge tacitness (often a result of hands-on experience and intimate familiarity with the processes involved), it is expected to be less prone to reverse engineering by imitators than product technology (Gopalakrishnan et al., 1999; Nieto and Pérez-Cano, 2004; Teece, 2003). Thus, in the case of process innovations, either a lower overall need for active protection efforts or at least a greater importance of secrecy protection may be observed. Moreover, since comprehensive innovators are more innovative in the broad sense (Baldwin and Gellatly, 2003), we expect a general preference of protection methods by these small firms. With regard to nascent innovators, it would be interesting to know whether innovation protection on the whole is considered less relevant.

In addition, we argue that the need for innovation protection is positively correlated to the degree of innovation. We therefore distinguish different kinds of significant innovations by

That is, WZ 93 (“Klassifikation der Wirtschaftszweige”), the German version of NACE Rev.1. 
creating four binary variables, whereby the first two constitute product innovations and the last two process innovations (for the following see Rammer et al., 2009). On the one hand, new-to-market innovations comprise recently introduced products or services that have not yet been supplied to the respective market segments. This implies that they do not necessarily need to be world firsts as innovations, but may gain their innovative character from market boundaries such as a regional business focus or a concentration on specific customer groups. New-to-firm innovations are product innovations that have no predecessors in a firm's range of products or services. They may be new-to-market innovations at the same time or simply an imitation of a competitor's product. Both types of significant product innovation may result in more profitability through an increased market share or the charging of higher prices. To be successful in this sense, however, firms need to take inventive steps that are clearly visible to their customers. Thus, in the case of new-to-market innovations in particular, we expect a high importance of innovation protection efforts. On the other hand, efficiency innovations are new processes that improve the cost efficiency of production processes (e.g. through advanced automation, simplification of procedures or the realization of synergies) and may result in increased price competitiveness among the innovating firms. Quality innovations are new processes that increase the quality of products or services and may therefore also permit higher sales. They are particularly relevant in service industries and often demand more precise and sometimes more time-consuming and costly production processes. Comparing the two kinds of significant process innovation, we suspect a relatively higher need to consciously protect quality innovation, since efficiency innovations are more indirectly related to outcomes. By contrast, quality innovations are in a sense outputs themselves, since they aim at reshaping production processes to better serve customer needs. Thus, in order to be successful, quality innovations must be more observable to the outside than efficiency innovations, which should in turn increase the corresponding risk of imitation.

The profiling variables of the degree of innovation already provide some indications of the relationship between firm performance and distinct modes of appropriability in the small enterprise sector. In fact, appropriation methods should not only help innovators thwart imitation but also contribute to achieving company goals, i.e. enable them to garner profits from their innovations (Hurmelinna-Laukkanen and Puumalainen, 2007). Differences in the utilization of innovation protection mechanisms may thus also be due to the fact that overall appropriation strategies target different company goals. Hence, to give further indications in this regard, a number of binary variables relating to the effects of innovation activities on firm performance are created. In this way, we are able to observe which product-oriented innovation effects (i.e. increased range of goods/services, entering of new markets, increased market share), which process-oriented ones (improved flexibility of production/service provision, increased capacity of production/service provision, reduced labour costs per unit output, reduced materials and energy per unit output) and which effects related to product as well as to process innovations (improved quality of goods/services) had a relevant impact on the economic performance of a small firm during the reference period. Thus, it is worth noting that we do not focus only on measurements of performance related to product innovations but also on the effects of significant process innovations. 
Thirdly, we investigate how innovation protection mechanisms in general and IPRs in particular complement the overall competitive strategy of small firms by lowering the threat of imitation by competitors. Several authors highlight the key role of sector-specific appropriability conditions in determining the endogenous relationship between market structure (e.g. firm size or the degree of concentration) and the rate of innovation (Cohen and Levin, 1989; Levin et al., 1985; Malerba and Orsenigo, 1993, 1997). Hence, the choice of appropriation strategies should - at least to some degree - be a rational response to external market conditions. We argue that this is especially true of small firms, since their management decisions are more straightforward in the sense that their scope for alternative strategies is limited (e.g. because of a lack of market power, a narrow customer and product base or a lack of resources or riskreducing behaviour among small business owners; see Wynarczyk et al., 1993) and because their appropriation strategies are likely to be handled by small business owners personally (Matthews et al., 2003). The evidence of Kitching and Blackburn (1998, 2003; see Section 1) already lends support to this argument, as it shows that small firms tend to adopt IPRs only under highly selective circumstances and are thus probably less likely than large firms to use IPRs routinely (for this argument, see also Arundel and Kabla 1998). Hence, we include diverse variables in the market environment of innovative small firms that may influence the use and importance of protection mechanisms.

To begin with, the geographical market orientation is of interest (export market activities or regional business focus). Focusing solely on the regional business environment may make it easier to develop high-trust relations with customers and suppliers, thereby lessening the need for protection efforts. The contrary may hold for firms with an international orientation, where maintaining patents could be a prerequisite for the successful penetration of export markets. Nonetheless, as Baldwin and Johnson (1996) point out, small firms that are more innovative are more likely to have an export orientation than those that are less innovative. Thus, the innovativeness of exporting SMEs may be associated with a greater emphasis on knowledge protection per se.

The general dynamism of the market environment may also play a key role in determining the perceived strength of single protection mechanisms. For example, methods such as secrecy and lead time advantage are likely to be far more effective in preventing imitation by competitors than patents in industries, where short product life cycles and a fast pace of technological advance are characteristic (Hall and Ziedonis, 2001; Hurmelinna-Laukkanen et al., 2008). To account for such dynamics, we used respondents' answers to the question of whether products and services mature rapidly (short product life cycle) and whether technologies change rapidly (fast pace of technology) in their main markets.

Next we used answers to questions on the ease of substitution and the number of main competitors to assess how innovation protection practices are related to the fact that small firms often follow a differentiation or niche strategy to overcome scale-related disadvantages in innovation. We created a binary variable that equals one if a small firm reports that its products are unlikely to be substituted by those of competitors. Another variable measures whether a small firm faces more than five main competitors. To assess whether a small firm tends to operate in a market where scale economies are important, another binary variable indicates whether its main competitors are larger than it in size. Furthermore, as Iversen (2003, 
2008) suggests, the relevance of IPRs in complementing a small firm's differentiation or niche strategy depends on the nature of the competition in the respective market. The author distinguishes three principal ways by which a firm can develop distinctiveness: by price, by technological performance, and in the eyes of the consumer. IPRs can help firms protect their distinct positions, either with respect to the underlying technical novelty (notably through patents or utility models) or by their distinctiveness as perceived by the customer (notably through trademarks or industrial designs). Thus, in the case of technological or design-based competition, protection by IPRs may serve as an effective instrument. On the other hand, IPRs have less significance in markets characterized by strong price competition, since selling efforts or technological performance play a lesser role in this context (Iversen, 2003, 2008). To account for such differences we use binary variables on the importance of price and non-price competition in the main markets of respondents (price, quality, technological edge, customer service and flexibility).

\section{Empirical analysis for the identification of appropriation modes}

\subsection{Types of innovation protection mechanism: factor analysis}

In the next subsection, we employ cluster analysis to examine whether innovative small firms form distinct groups with respect to their overall appropriation strategy. For two reasons, we used the results of a factor analysis as clustering variables to compress the eight primary variables into distinct types of innovation protection mechanism. First of all, the grouping of correlated variables into factors avoids the overweighting of single variable sets (i.e. different types of protection mechanism). Secondly, since factor scores are a linear combination of weighted individual variables, they are likely to represent more robust variables than the originals (Hair et al., 1998). Two standard measures are used to ensure that the primary variables are sufficiently correlated with each other to justify the application of factor analysis. Bartlett's test of sphericity $(2051.39, \mathrm{p}<0.000)$ and the Kaiser-Meyer-Olkin Measure of Sampling Adequacy $(\mathrm{KMO}=0.749)$ both show satisfactory results. Furthermore, we apply the latent root criterion to decide on the number of factors, since it is the most commonly used approach for this aim. Accordingly, the number of factors to extract equals the number of factors with eigenvalues greater than one.

By using standard factor analysis (principal component factoring and varimax rotation), a three-factor solution is found, explaining $65 \%$ of the variance. The rotated factor loadings are shown in Table A2 (see Appendix). The first factor accounts for the most variance and is marked by high loadings on all three informal protection mechanisms. It is thus labelled 'Informal methods'. The importance of patents and utility models for the appropriation of innovation returns loads significantly on Factor 2, whereas Factor 3 shows high loadings on industrial designs and copyrights. Patents and utility models are sought to protect new solutions to technical problems, allowing them to be summarized under the heading 'Technical IPRs'. On the other hand, industrial designs and copyrights can be described as 'Other IPRs', since, although their protection can also be related to technology-oriented areas, the subject of their protection does not lie in technical novelty. Instead, protection is given to the visual ap- 
pearance of products (industrial design) and artistic creations such as books, blueprints or computer programs (copyright). The picture is less clear in the case of trademarks, however. The respective variable loads equally on the second factor and on the third. Initially, one would expect only higher loadings on the latter, since trademarks do not actually constitute a technical IPR either. They represent a sign (or combination of signs) that helps customers distinguish the products or services from those of competing firms. Yet, the loading on the second factor, as well as that on the third, is not surprising, since trademarks in particular can be effectively combined with technical IPRs. The additional use of trademarks might create brand equity for patenting firms ("Technology Brands") and significantly increase their appropriation of the returns generated by intangible technological assets (Jennewein, 2005; Ramello and Silva, 2006).

\subsection{Identification and interpretation of appropriation strategies: cluster analysis}

The results of the factor analysis already indicate that at least some innovation protection mechanisms are not mutually exclusive but rather complement each other effectively. To gain a better understanding of this aspect we carried out a cluster analysis of the three factors to identify different modes of appropriability. As suggested by Hair et al. (1998), we combined hierarchical and non-hierarchical methods of cluster analysis to gain the benefits of each. In a first step, hierarchical techniques were used to identify outliers and determine the number of clusters. Ward's algorithm with squared Euclidean distance as the measure of similarity led to a 4-Cluster solution. ${ }^{6}$ Prior to this we used the single linkage method to identify outliers, since this procedure is particularly prone to chaining. The corresponding dendrogram caused us to delete six observations as outliers, reducing the dataset to 1,251 firms. In a second step, to adjust the hierarchical results, the cluster centroids from the Ward method served as initial seed points for a k-means (non-hierarchical) cluster procedure. Table 2 shows the final cluster solution using the original values of the primary variables. A non-hierarchical cluster analysis with random seeds led to very similar results, demonstrating the stability of the derived solution. The robustness of the cluster results were further corroborated by a Kruskal-Wallis test, which examined whether the clusters were really distinctive in view of the primary variables. It revealed that the average values of the latter differed significantly across the four clusters.

Thus, according to the cluster results (see Table 2), four modes of appropriability are prevalent in the small enterprise sector. The ‘informal protection group’ (Cluster 1), comprising 241 innovative small firms (19\% of the total sample), is characterized by the highest scores in all three informal protection methods. IPRs, on the other hand, seem to have almost no relevance for the appropriation of innovation returns in this group. Maintaining a lead time advantage over competitors is thereby considered as most important. Firms in the informal protection group, however, also assign on average a higher relevance to secrecy and the com-

Apart from a visual inspection of the dendrogram we used the results from two stopping rules implemented in our statistical software (Calinski-Harabasz pseudo-F index and the Duda-Hart Je(2)/Je(1) index) to determine the number of clusters. 
plexity of design. A distinctive feature of the 'patent-oriented group' (Cluster 2), of 139 members (11\% of the total sample), is that technical IPRs (especially patents) are seen to be important for the protection of innovation results. Trademarks are also relevant for these innovators, which confirms the special role of trademark protection as discussed above.

\section{Table 2}

Cluster solution: mean values of the primary variables and statistical significance of cluster differences (Kruskal-Wallis test)

\begin{tabular}{|c|c|c|c|c|c|c|}
\hline & \multicolumn{4}{|c|}{ Cluster of innovative small firms } & \multirow{2}{*}{ d.f. } & \multirow{2}{*}{$\begin{array}{l}\text { Chi- } \\
\text { Square }\end{array}$} \\
\hline & $\begin{array}{c}1 \\
(n=241)\end{array}$ & $\begin{array}{c}2 \\
(n=139)\end{array}$ & $\begin{array}{c}3 \\
(n=71)\end{array}$ & $\begin{array}{c}4 \\
(n=800)\end{array}$ & & \\
\hline Patent & 0.30 & 2.25 & 0.75 & 0.02 & 3 & $259.2 * *$ \\
\hline Utility model & 0.11 & 1.56 & 0.66 & 0.03 & 3 & $146.3^{* *}$ \\
\hline Industrial design & 0.00 & 0.03 & 0.44 & 0.00 & 3 & $26.7 * *$ \\
\hline Trademark & 0.35 & 1.01 & 1.28 & 0.13 & 3 & $98.1^{* *}$ \\
\hline Copyright & 0.02 & 0.00 & 2.04 & 0.01 & 3 & $177.7 * *$ \\
\hline Secrecy & 2.26 & 2.15 & 1.48 & 0.17 & 3 & $487.7 * *$ \\
\hline $\begin{array}{l}\text { Complexity of } \\
\text { design }\end{array}$ & 1.78 & 0.48 & 1.06 & 0.01 & 3 & $296.1^{* *}$ \\
\hline $\begin{array}{l}\text { Lead time } \\
\text { advantage }\end{array}$ & 2.62 & 2.04 & 1.62 & 0.24 & 3 & $510.0 * *$ \\
\hline Share of sample & $19 \%$ & $11 \%$ & $6 \%$ & $64 \%$ & & \\
\hline
\end{tabular}

Note: Values are means of Likert scale responses $(0=$ no utilization, $1=$ low importance, $2=$ moderate importance, 3 = high importance)

** report a significance level of $1 \%$

Apart from technical IPRs, firms in the patent-oriented group also attribute higher importance to both secrecy and lead time advantage (albeit lower than in Cluster 1), but not to complexity of design. This finding is of interest for two reasons: firstly, secrecy is often seen as the strategic alternative to patent protection with its involved disclosure requirement (Granstrand, 1999; Hussinger, 2004; Nieto and Pérez-Cano, 2004). Yet, our results imply that small firm innovators either make use of both protection mechanisms simultaneously (e.g. by patenting the codified components of an innovation and keeping core technological know-how secret) or employ them at different stages of the innovation process during the reference period (for example, patents may be highly effective in the commercialization stage, while secrecy may be more relevant in the early stages of the innovation process; see Cohen et al. 2000). ${ }^{7}$ Secondly, patents and the complexity of design are instead used as substitutes, which

\footnotetext{
7 It might be argued, of course, that respondents referred to the protection of different innovation projects. Yet, since smaller firms are less likely to conduct different innovation projects concurrently we are of the opinion that this argument has less weight in the present context.
} 
corroborates the findings of Amara et al. (2008). ${ }^{8}$ On the one hand, it can be argued that this is due to the fact that innovations in the first cluster are less patentable than those in the second and it explains why these firms compensate by using a different protection mechanism. In fact, in the case of frequent incremental improvements, complex product design is an informal, technology-based protection method ideally suited to reducing the opportunity of imitation by competitors (Päällysaho and Kuusisto, 2008; for a small firm perspective see Mazzarol and Reboud, 2009). Conversely, however, firms in the first cluster do not show a higher preference for utility models (also referred to as "petty patents" - see Footnote 3). Hence, the assurance that the sheer complexity of the technological knowledge and corresponding learning effects will dissuade potential imitators may be a further reason why a number of small firms in the informal protection group do not seek out IPRs. Small firms may therefore also forgo expensive patents because they simply see no need for their use.

The 'copyright-oriented group' (Cluster 3), with 71 firms (6\% of the total sample), is characterized by a higher relevance of other than technical IPRs. Copyright protection in particular serves as an important protection mechanism for these innovators. Compared with the other groups, trademarks and industrial design are more important to them in innovation protection. The relatively low score on the industrial design variable is puzzling at first (see also Table 1). However, this is probably due to the fact that in order to harmonize the German Innovation Survey with the CIS questionnaire, the term 'Industriedesign' was used instead of the more common term 'Geschmacksmuster' (Aschhoff et al., 2007). This must have caused some confusion among respondents, so a higher relevance of industrial design in Cluster 3 can be assumed. The greater importance of certain IPRs to innovators in the copyright-oriented group is accompanied by the use of all three informal protection methods; although it should be noted that the corresponding score levels in all cases are lower than those in the first cluster. All in all, the use of innovation protection mechanisms by firms in the third cluster seems slightly less selective than that observed in the first and second groups (e.g. because the corresponding firms also indicate little preference for technical IPRs). Finally, the results for the 'non-protection group' (Cluster 4) reveal a striking feature of the small enterprise sector: by far the most innovative small firms (64\% of the total sample) made no conscious effort to protect their innovations during the reference period. Thus, in view of the methods under consideration it seems that innovators in Cluster 4 either face a lower risk of imitation than those in the remaining three groups or other factors are responsible for innovation protection efforts playing only a minor role.

\section{Modes of appropriability: validation and profiling}

The results given in Table 3 confirm the assumption that in-house R\&D activities, an engagement in innovation-related cooperation and the intensity of innovation expenditure are key drivers of variability in the appropriation strategies adopted by innovative small firms. We see significant differences in these variables across the four clusters identified in the pre-

8 The mutually exclusive nature of technical IPRs and complexity of design is also shown in Table A2 (see Appendix). 
vious section, leading us to the conclusion that the derived appropriation modes have a predictive validity. Firms in the patent-oriented group conduct in-house $R \& D$ on a permanent basis much more often than those in the other groups, have a stronger focus on cooperative innovation and tend to be concentrated at the highest level of innovation expenditure. Generally, the results point to a high degree of innovativeness in these firms. The contrary is evident in the non-protection group, where the corresponding firms show by far the lowest level in the first two variables and tend to spend a relatively small percentage of their monetary resources on innovation activities. Thus, when it comes to these indicators, the second and the fourth modes are in a sense complete opposites. Clusters 1 and 3 display values in between this range, whereby members of the former group are, on average, more inclined to continuous R\&D activities, cooperative innovation and a higher intensity of innovation expenditure. Since the basic condition for protection under the patent system is a high degree of inventive novelty, it is evident that the less frequent use of technical IPRs by small firms may often result from a lack of this requirement because of the incremental nature of their innovations. At the same time, however, a relatively low level of investment in innovation might in many cases also reduce the need for expensive patents to appropriate sufficient rates of return on such activities. Yet, contrary to what might be expected, a large number of innovative small firms (given the relative size of Cluster 4) do not switch to informal protection methods, but forgo any attempt at innovation protection at all.

\section{Table 3}

Predictive validity of selected variables: percentage share per cluster and statistical significance of cluster differences (Pearson's chi-square test)

\begin{tabular}{|c|c|c|c|c|c|c|}
\hline & \multirow[t]{2}{*}{ Total } & \multicolumn{4}{|c|}{ Cluster } & \multirow{2}{*}{$\begin{array}{l}\text { Chi- } \\
\text { Square }\end{array}$} \\
\hline & & 1 & 2 & 3 & 4 & \\
\hline Permanent in-house $R \& D$ & 33 & 56 & 75 & 37 & 16 & $245.26^{* *}$ \\
\hline Cooperative innovation & 21 & 34 & 58 & 25 & 11 & $183.56^{* *}$ \\
\hline \multicolumn{7}{|c|}{ Total innovation expenditure (\% of turnover) } \\
\hline $0 \% \leq x<1 \%$ & 23 & 7 & 9 & 16 & 31 & \\
\hline $1 \% \leq x<5 \%$ & 32 & 27 & 24 & 26 & 36 & \\
\hline $5 \% \leq x<15 \%$ & 23 & 31 & 21 & 34 & 20 & \\
\hline$x \geq 15 \%$ & 22 & 35 & 46 & 24 & 13 & $137.59 * *$ \\
\hline
\end{tabular}

Note: The table shows the corresponding usage share for each cluster. For example, 56\% of firms in Cluster 1 conducted in-house R\&D on a permanent basis in 2002-2004.

** report a significance level of $1 \%$

In order to characterize each mode of appropriability, Tables 4 - 6 show descriptive profiles in three relevant dimensions (industry composition by knowledge intensity; output of innovation; features of the market environment). By examining whether the four clusters also differ in terms of the profiling variables, we gain further information on the characteristics of innovative small firms that choose a certain appropriation strategy. Table 4 shows that each appropriation mode is distributed across all of the four industry sectors under consideration. 
However, the industry composition of individual modes appears to be concentrated in certain ways. Members of the first three groups are rarely found in service industries with low knowledge intensity. Instead, the non-protection group consists of an above-average share of firms belonging to this industry sector. Furthermore, the industry composition of Clusters 1 and 2 is more strongly concentrated than in other groups. The majority of small firms in the informal protection group operate in knowledge-intensive service sectors. A smaller, albeit sizable, share of this group is also found in knowledge-intensive manufacturing industries. More or less the opposite is true of the patent-oriented group, while only a small share of firms belonging to the non-protection group are found in knowledge-intensive manufacturing industries. The industry composition of the fourth mode is distributed more evenly across the other industry sectors. Firms in the copyright-oriented group are well represented in knowledge-intensive service industries but are also frequently found in manufacturing industries with low knowledge intensity. To sum up, the results in Table 4 indicate that small firms that consider innovation protection mechanisms important tend to be concentrated in more innovative industries.

\section{Table 4}

Knowledge intensity of industries, percentage share per cluster and statistical significance of cluster differences (Pearson's chi-square test)

\begin{tabular}{lc|cccccc}
\hline & Total & \multicolumn{2}{c}{ Cluster } & & & \multirow{2}{*}{$\begin{array}{l}\text { Chi- } \\
\text { Square }\end{array}$} \\
\cline { 3 - 6 } & & & 1 & 2 & 3 & 4 & \\
\hline Industry Sector & & & & & & \\
Knowledge-intensive manufacturing & 22 & 30 & 43 & 24 & 16 & \\
Other manufacturing & 26 & 20 & 22 & 32 & 28 & \\
Knowledge-intensive services & 34 & 41 & 33 & 37 & 31 & \\
Other services & 18 & 9 & 2 & 7 & 25 & $114.09 * *$ \\
\hline
\end{tabular}

Notes: The table shows the corresponding usage share for each cluster and the total sample. For example, $30 \%$ of small firms in Cluster 1 belong to manufacturing industries that are classified as knowledge-intensive.

** report a significance level of $1 \%$

From the information displayed in Table 5, it is evident that a small firm's mode of appropriability varies strongly with the type of innovator, the underlying degree of novelty and the corresponding effects on firm performance. The composition of each cluster according to basic innovator type indicates, once again, a stronger concentration of the first and second modes. Both clusters consist of above-average shares of product-only innovators and comprehensive innovators. Furthermore, compared with the other groups, the patent-oriented group has the lowest share of process-only innovators. In contrast, the fourth mode is characterised by an above-average share of this type of innovator. This finding lends support to the assumption that, because of its lower observability and codifiability, process technology is less likely to be patented than product technology. Yet, because of this, process-only innovators are apparently more inclined to forgo any kind of innovation protection method than to show a higher preference for secrecy (as is often assumed in order to explain the less frequent use of 
patents by process innovators; see, for example, Arundel, 2001; Hanel, 2008; Nieto and Pérez-Cano, 2004). The non-protection group is further characterized by a relatively large share of nascent innovators. This indicates that, for some firms choosing the fourth mode, the use of protection methods may be less relevant because they have not yet completed their current innovation projects. These results demonstrate that, depending on their specific types of innovation, small firms tend to be selective in the adoption of appropriation strategies.

\section{Table 5}

Output of innovation, percentage share per cluster and statistical significance of cluster differences (Pearson's chi-square test)

\begin{tabular}{|c|c|c|c|c|c|c|}
\hline & \multirow[t]{2}{*}{ Total } & \multicolumn{4}{|c|}{ Cluster } & \multirow{2}{*}{$\begin{array}{l}\text { Chi- } \\
\text { Square }\end{array}$} \\
\hline & & 1 & 2 & 3 & 4 & \\
\hline \multicolumn{7}{|l|}{ Type of innovator } \\
\hline Product-only innovator & 38 & 43 & 46 & 39 & 35 & \\
\hline Process-only innovator & 17 & 7 & 4 & 13 & 23 & \\
\hline Comprehensive innovator & 32 & 42 & 40 & 34 & 28 & \\
\hline Nascent innovator & 12 & 8 & 10 & 14 & 14 & $69.67 * *$ \\
\hline \multicolumn{7}{|l|}{ Degree of innovation } \\
\hline Introduction of new-to-market innovations & 36 & 48 & 67 & 50 & 21 & $106.23 * *$ \\
\hline Introduction of new-to-firm innovations & 48 & 58 & 66 & 65 & 38 & $46.33^{* *}$ \\
\hline Introduction of efficiency innovations & 42 & 44 & 49 & 44 & 40 & 2.02 \\
\hline Introduction of quality innovations & 64 & 78 & 68 & 71 & 59 & $14.07 * *$ \\
\hline \multicolumn{7}{|l|}{ Effects of innovation } \\
\hline Increased range of goods/services & 75 & 88 & 87 & 81 & 69 & $47.42^{* *}$ \\
\hline Entering of new markets & 57 & 68 & 74 & 68 & 50 & $46.93 * *$ \\
\hline Increased market share & 50 & 63 & 66 & 66 & 41 & $63.21 * *$ \\
\hline Improved quality in goods/services & 76 & 83 & 75 & 81 & 73 & $10.33 *$ \\
\hline $\begin{array}{l}\text { Improved flexibility of production/service } \\
\text { provision }\end{array}$ & 61 & 62 & 50 & 69 & 62 & $9.20 *$ \\
\hline $\begin{array}{l}\text { Increased capacity of production/service } \\
\text { provision }\end{array}$ & 45 & 47 & 37 & 46 & 45 & 3.66 \\
\hline Reduced labour costs per unit output & 31 & 34 & 28 & 34 & 30 & 2.23 \\
\hline $\begin{array}{l}\text { Reduced materials and energy per unit } \\
\text { output }\end{array}$ & 20 & 22 & 25 & 22 & 18 & 3.66 \\
\hline
\end{tabular}

Note: The table shows the corresponding usage share for each cluster and the total sample. For example, $42 \%$ of small firms in Cluster 1 are classified as product-only innovators.

** report a significance level of $1 \%$, * of $5 \%$

Table 5 further confirms that the decision by small firms whether to use innovation protection methods or not is, at least in part, a function of their innovativeness. On average, members of the non-protection group tend less often to introduce any kind of significant innovation. In the case of product innovations, however, the results are far more striking. A focus on 
new-to-market innovations is a specific characteristic of the patent-oriented group, which again points to the innovativeness of these firms. The opposite is true of firms in the fourth group. Above-average shares of new-to-market innovations are also found in the first and third clusters. In the case of new-to-firm innovations the results are similar but less pronounced, possibly because they may also be imitations of competitors' products. On the other hand, there is little variation in appropriation modes in the case of efficiency innovations, which suggests that, through a lower overall risk of imitation, this kind of significant process innovation requires less protection. Hence, the relatively high degree of tacitness of knowledge that is typically embodied in efficiency innovations may often be sufficient in itself to successfully appropriate the corresponding returns. Quality innovations seem to be especially important for members of the informal protection group. As with the results for efficiency innovations, this is worth noting, since it may also imply that other appropriation strategies function equally or even more effectively for this kind of significant process innovation than those based, for example, on patent protection.

Table 5 elaborates on the latter results by further showing the relationship between the identified appropriation modes and various effects of innovation on firm performance. In this way it is confirmed that, compared with the non-protection mode, all other appropriation strategies are related to higher shares of respondents reporting product-oriented effects (increased range of goods/services, entering of new markets, increased market share) having a relevant impact. We interpret this finding as indicating that the use of formal and informal protection methods in general is likely to assist small firms in appropriating higher returns from their product innovations. However, as the above results already suggest, the same may not hold true for the appropriability of process innovations. Much less variation is observed between the fourth and the other groups in the case of process-related innovation effects, a finding that is not surprising, given the relatively high number of efficiency and quality innovations and the above-average share of process-only innovators in the non-protection group. Hence, concerning the appropriation of process innovation returns, our results indicate that the fourth mode is not necessarily less effective than other appropriation strategies.

With respect to the relevance of product-oriented innovation effects, Table 5 also indicates that the first three appropriation modes do not differ much from each other, although it has to be noted that innovation activities in the patent-oriented group are more strongly related to the entering of new markets. Thus, depending on their specific business contexts, both IPR-based and informal appropriation strategies seem to work effectively for product-innovating small firms. Yet, it is worth noting that the patent-oriented group is characterized in almost all cases by a relatively lower share of respondents reporting process-related innovation effects having a relevant impact on their firm performance. Perhaps most interesting in this regard are the improvement of quality in goods and services and the better production and service provision in terms of flexibility and capacity as outcomes of innovation. In fact, the ability to offer superior customer service and to adjust product quality to meet particular customer needs in a fast, flexible and incremental way is often seen as a distinct advantage of small firms in their innovation efforts (see Section 1). Since even the non-protection group is about average with respect to these innovation effects, we interpret this finding (together with the results of Table 3) as an indication that the less frequent use of technical IPRs by smaller firms should also be 
related to the specific nature of their innovation regime, an argument further supported by Table 6.

To begin with, as expected, the appropriation strategies chosen by innovative small firms tend to be aligned to their market environments (see Table 6). One striking feature is that firms actively engaged in innovation protection usually operate in markets other than those of firms in the non-protection group. A higher percentage of them are engaged in exports, whereas many members of the non-protection group focus solely on their regional business environment. Small firm innovators who choose one of the first three modes also face a more dynamic market environment in terms of the product life cycle and the pace of technology. In addition, these small firms tend to have larger counterparts, which is probably why they are less able to compete on price than firms in the non-protection group. Thus, to overcome scalerelated disadvantages in innovation, many small firms in the first three groups seem to pursue a differentiation or niche strategy (their products are more difficult to substitute and they have fewer main competitors), complemented by the use of several methods to protect this distinctiveness. On the other hand, members of the non-protection groups more often face strong price competition than other firms, a further explanation of why innovation protection efforts have low relevance in this group (protection methods such as IPRs have less applicability in cases where firms compete on price only).

In Table 6 we see that there is also some variation between the first three modes, which indicates a higher effectiveness of certain protection methods in matching specific market environments. A larger number of small firms in the patent-oriented group are active in export markets, which once again reflects their innovative abilities. Moreover, maintaining patents is probably a prerequisite for successful entry into foreign markets, a fact that may explain the relatively high importance of the entering of new markets as an innovation effect in this group (see Table 5). Yet, under certain circumstances protection methods other than patents seem to be preferred. For example, the high emphasis on informal protection methods in the first group should also be due to these firms are tending to face shorter product life cycles, making lead time or secrecy particularly effective. It seems that this finding also holds true for the pace of technological advance but the cluster differences are slightly less pronounced in this case. Furthermore, logically enough, small firms in the copyright-oriented group seldom compete on technological advances compared with members of the first two appropriation modes. On the contrary, the strong science base of the patent-oriented group is corroborated by the fact that it consists of a strong above-average share of small firms showing a commitment to technological competition. In all groups, more or less the same proportion of innovators tend to operate in markets where quality of product and service are vital to remaining competitive, which is why no significant differences between clusters are found in this regard. However, the informal protection and non-protection groups are characterized by above-average shares of small firms with a strong customer orientation. This finding is of special interest for two reasons. Firstly, in line with the above discussion, it indicates that complementary assets such as customer-oriented service and manufacturing capabilities may often carry more weight than the adoption of (technical) IPRs when it comes to the successful appropriation of innovation returns in the small enterprise sector. Secondly, it points to a further reason why complexity of design rather than patent protection works effectively for a number of small 
firms. Usually less standardized and - based on firm-specific skills and competence in problem solving - frequently tailored to meet individual customer needs, small firm innovations are often likely to go hand in hand with higher costs and more time for reverse-engineering from the perspective of the potential imitator, all of which makes the complexity of design an effective appropriation method and lowers the need for patent protection.

\section{Table 6}

Market environment, percentage share per cluster and statistical significance of cluster differences (Pearson's chi-square test)

\begin{tabular}{|c|c|c|c|c|c|c|}
\hline & \multirow[t]{2}{*}{ Total } & \multicolumn{4}{|c|}{ Cluster } & \multirow{2}{*}{$\begin{array}{l}\text { Chi- } \\
\text { Square }\end{array}$} \\
\hline & & 1 & 2 & 3 & 4 & \\
\hline \multicolumn{7}{|l|}{ Geographical markets } \\
\hline Export market activities & 37 & 49 & 73 & 45 & 27 & $127.58^{* *}$ \\
\hline Regional business focus & 25 & 14 & 4 & 17 & 33 & $76.47^{* *}$ \\
\hline \multicolumn{7}{|l|}{ Features of competition } \\
\hline Short product life cycle & 37 & 47 & 38 & 41 & 33 & $15.54^{* *}$ \\
\hline Fast pace of technology & 46 & 53 & 48 & 49 & 43 & $8.26^{*}$ \\
\hline Difficult substitution & 37 & 42 & 49 & 47 & 32 & $23.74 * *$ \\
\hline \multicolumn{7}{|l|}{ Main competitors } \\
\hline More than 5 main competitors & 39 & 33 & 33 & 34 & 43 & $10.51^{* *}$ \\
\hline Main competitors are larger in size & 46 & 50 & 57 & 54 & 43 & $13.00^{* *}$ \\
\hline \multicolumn{7}{|l|}{ Important factors for competition } \\
\hline Price & 43 & 33 & 34 & 36 & 49 & $27.16^{* *}$ \\
\hline Technological edge & 12 & 21 & 25 & 13 & 7 & $54.35^{* *}$ \\
\hline Quality & 44 & 44 & 49 & 47 & 43 & 2.01 \\
\hline Customer service and flexibility & 27 & 30 & 17 & 17 & 29 & $13.94 * *$ \\
\hline
\end{tabular}

Notes: The table shows the corresponding usage share for each cluster and the total sample. For example, $49 \%$ of small firms in Cluster 1 were active in export markets in 2002-2004.

** report a significance level of $1 \%$, * of $5 \%$

\section{Conclusion and implications for policy and research}

This paper has examined various modes of appropriability in the small enterprise sector. Our empirical analysis revealed that innovative small firms can be divided into four distinct groups according to the kind of appropriation strategy they adopt. One finding is that for many innovative small firms the main issue is not whether to use IPRs or not, but whether to protect their innovations from imitation at all. Furthermore, the results show that formal and informal protection mechanisms should not be seen as mutually exclusive, since small firm innovators combine several methods to form their appropriation strategies. With the exception of the non-protection group, informal protection mechanisms play a significant role in all appropriation modes. Yet this is only true in the case of secrecy and lead time advantage, since these two methods are either complemented by technical and other IPRs or are used in 
combination with complexity of design as a technology-based informal protection method. Hence, our findings do not imply that patents are of low relevance to the protection of small firm innovations in general. But it is evident that only in a lesser proportion of the small enterprise sector is the use of patents (in combination with other formal and informal protection mechanisms) perceived to be of significant importance for the appropriation of innovation returns.

The validation and profiling of the identified appropriation modes has shown that the relevance of various protection methods can be explained to a large extent by their availability and efficacy in specific business contexts (for a brief overview see Table 7). As expected, the degree of innovativeness plays a major role. Members of the patent-oriented group are much more innovative than other small firms in terms of indicators such as continuity of in-house $\mathrm{R} \& \mathrm{D}$, cooperative innovation or the introduction of new-to-market innovations. Hence, given the relatively small size of this cluster, patents may be less available for a large number of small firms as they are more likely to innovate incrementally. However, at the same time, a lower level of investment in innovation might in many cases also reduce the need for expensive patents in appropriating sufficient rates of return on innovation activities. In line with the latter argument, our results further indicate that the relevance of each appropriation mode also depends on other factors (e.g. the type of innovator, the kind of significant innovation, and corresponding effects on firm performance or the general market environment) that under certain circumstances even make the non-protection mode a reasonable choice.

With respect to the degree of innovation and corresponding effects on small firm performance, we have found some indications that each appropriation mode - depending on the object of protection - can be effective regarding company goals. These results have given us deeper insights into the kinds of innovation that are protected by certain types of protection mechanism. Appropriation strategies that employ informal protection methods only or also use IPRs seem to be much more likely to aid small firms in appropriating higher returns from their product innovations. At this point, the first three appropriation modes do not differ much from each other, which suggests that they work equally effectively for product-innovating small firms in a variety of business contexts. Yet, when it comes to the appropriation of process innovation returns, our results indicate that the non-protection mode is not necessarily less effective than other appropriation strategies. Furthermore, the patent-oriented appropriation mode has been observed to be less characterized by measures typically associated with innovation in most small firms (e.g. with respect to certain process-related innovation effects and customer orientation as a success factor in securing and sustaining a competitive advantage) than, for example, the first appropriation mode with its strong emphasis on informal protection methods. Thus, we conclude that apart from cost and complexity issues, the low propensity of smaller firms to adopt IPRs should also be related to the specific nature of their innovation regime. In summing up, this study implies that the use of IPRs by innovative small firms is highly selective. This by no means ignores the fact that IPRs can be a crucial factor in their appropriation strategy. However, many small firm innovators do not perceive IPRs to be important, since they either forgo active protection efforts entirely or find alternative means of protection more relevant to the successful appropriation of innovation returns. 
Table 7

Classification of innovative small firms according to their mode of appropriability

\begin{tabular}{|c|c|c|c|c|}
\hline Cluster & 1 & 2 & 3 & 4 \\
\hline \multicolumn{5}{|l|}{ Appropriation strategy } \\
\hline $\begin{array}{l}\text { Protection mechanisms } \\
\text { (dominant ones are } \\
\text { marked in bold) }\end{array}$ & $\begin{array}{l}\text { Secrecy, complexity } \\
\text { of design, lead time }\end{array}$ & $\begin{array}{l}\text { Patent, utility } \\
\text { model, trademark, } \\
\text { secrecy, lead time }\end{array}$ & $\begin{array}{l}\text { Trademark, copy- } \\
\text { right, secrecy, com- } \\
\text { plexity of design, } \\
\text { lead time }\end{array}$ & $\begin{array}{l}\text { No usage of inno- } \\
\text { vation protection } \\
\text { mechanisms }\end{array}$ \\
\hline Share $(\mathrm{N}=1251)$ & $19 \%$ & $11 \%$ & $6 \%$ & $64 \%$ \\
\hline \multicolumn{5}{|l|}{ Input of innovation } \\
\hline In-house R\&D & Medium frequency & High frequency & Medium frequency & Low frequency \\
\hline Cooperative innovation & Medium frequency & High frequency & Medium frequency & Low frequency \\
\hline $\begin{array}{l}\text { Expenditure on } \\
\text { innovation }\end{array}$ & Medium intensity & High intensity & Medium intensity & Low intensity \\
\hline Industry sector & $\begin{array}{l}\text { Tends to be concen- } \\
\text { trated on knowledge- } \\
\text { intensive services }\end{array}$ & $\begin{array}{l}\text { Tends to be concen- } \\
\text { trated on knowledge- } \\
\text { intensive manufac- } \\
\text { turing }\end{array}$ & $\begin{array}{l}\text { Well represented in } \\
\text { all sectors apart from } \\
\text { non-knowledge- } \\
\text { intensive services }\end{array}$ & $\begin{array}{l}\text { Above average } \\
\text { share of non- } \\
\text { knowledge- } \\
\text { intensive services }\end{array}$ \\
\hline \multicolumn{5}{|l|}{ Output of innovation } \\
\hline Type of innovator & $\begin{array}{l}\text { Comprehensive } \\
\text { innovators } \\
\text { Product-only } \\
\text { innovators }\end{array}$ & $\begin{array}{l}\text { Comprehensive } \\
\text { innovators } \\
\text { Product-only } \\
\text { innovators }\end{array}$ & $\begin{array}{l}\text { All types of product } \\
\text { and process } \\
\text { innovators } \\
\text { Nascent innovators }\end{array}$ & $\begin{array}{l}\text { Above-average } \\
\text { share of process- } \\
\text { only innovators } \\
\text { Nascent innovators }\end{array}$ \\
\hline $\begin{array}{l}\text { Degree and effects of } \\
\text { innovation }\end{array}$ & Quality innovations & $\begin{array}{l}\text { New-to-market } \\
\text { innovations } \\
\text { New-to-firm } \\
\text { innovations } \\
\text { Relatively low rele- } \\
\text { vance of most effects } \\
\text { related to process } \\
\text { innovations }\end{array}$ & $\begin{array}{l}\text { New-to-firm } \\
\text { innovations }\end{array}$ & $\begin{array}{l}\text { Particularly less } \\
\text { engaged in signi- } \\
\text { ficant product } \\
\text { innovation } \\
\text { Below average re- } \\
\text { levance of product- } \\
\text { oriented innova- } \\
\text { tion effects }\end{array}$ \\
\hline \multicolumn{5}{|l|}{ Market environment } \\
\hline Geographical markets & Intermediate position & $\begin{array}{l}\text { Strong international } \\
\text { orientation }\end{array}$ & Intermediate position & $\begin{array}{l}\text { Strong regional } \\
\text { business focus }\end{array}$ \\
\hline Features of competition & $\begin{array}{l}\text { Medium difficulty of } \\
\text { substitution } \\
\text { Shorter product life } \\
\text { cycles } \\
\text { Faster pace of tech- } \\
\text { nology }\end{array}$ & $\begin{array}{l}\text { Difficult substitution } \\
\text { Intermediate position } \\
\text { in terms of market } \\
\text { dynamism }\end{array}$ & $\begin{array}{l}\text { Difficult substitution } \\
\text { Intermediate position } \\
\text { in terms of market } \\
\text { dynamism }\end{array}$ & $\begin{array}{l}\text { Easy substitution } \\
\text { Longer product life } \\
\text { cycles } \\
\text { Slower pace of } \\
\text { technology }\end{array}$ \\
\hline Main competitors & $\begin{array}{l}\text { Smaller number of } \\
\text { main competitors } \\
\text { Intermediate position } \\
\text { in terms of main } \\
\text { competitors' relative } \\
\text { firm size }\end{array}$ & $\begin{array}{l}\text { Smaller number of } \\
\text { main competitors } \\
\text { Main competitors } \\
\text { often larger in size }\end{array}$ & $\begin{array}{l}\text { Smaller number of } \\
\text { main competitors } \\
\text { Main competitors } \\
\text { often larger in size }\end{array}$ & $\begin{array}{l}\text { Larger number of } \\
\text { main competitors } \\
\text { Main competitors } \\
\text { often similar in } \\
\text { size }\end{array}$ \\
\hline $\begin{array}{l}\text { Important factors for } \\
\text { competition }\end{array}$ & $\begin{array}{l}\text { Non-price com- } \\
\text { petition } \\
\text { Technological edge } \\
\text { Customer service } \\
\text { and flexibility }\end{array}$ & $\begin{array}{l}\text { Non-price com- } \\
\text { petition } \\
\text { Technological edge }\end{array}$ & $\begin{array}{l}\text { Non-price com- } \\
\text { petition }\end{array}$ & $\begin{array}{l}\text { Price competition } \\
\text { Customer service } \\
\text { and flexibility }\end{array}$ \\
\hline
\end{tabular}

Note: The assignment of attributes to the classification categories was oriented on the observed specificities of each appropriation mode in comparison with the others. Thus, for the sake of overview, only key characteristics are highlighted. 
Our results have several implications for innovation policies that target small businesses. First of all we were able to account for the heterogeneity among innovative small firms when it comes to different appropriation strategies. In this way, policy makers aiming to improve the appropriability conditions of smaller firms are in a better position to meet the specific needs of certain subgroups of SMEs. For example, it is important to be aware that innovative small firms effectively combine the use of secrecy and lead time either with technical IPRs or with complexity of design. Policy attempts to foster the use of IPRs by SMEs should therefore not be based on the argument that small firms might have difficulty in coping with the IPR system, since they are, on average, more likely to use informal methods such as secrecy or lead time advantage. Moreover, this study indicates that the less frequent use of IPRs by smaller firms should not necessarily give rise to public concern since it is also related to the specific nature of their innovation regime. As a result, non-IPR-oriented appropriation strategies are often a reasonable choice for small firm innovators, which implies that easier and cheaper access to IPRs does not necessarily lead to a higher use by SMEs. Moreover, the fostering of IPR protection in such parts of the small enterprise sector that do not depend on such means of protection in terms of appropriability may make it harder for corresponding companies to profit from knowledge-sharing activities and thus may inhibit further innovation (see Hurmelinna et al., 2007). Hence, although this does not make policy attempts to enhance the effective use of IPRs by SMEs irrelevant, policy-makers should nevertheless bear in mind that a corresponding target group may be more limited than might be expected at first glance. Either way, the decision of innovative small firms to use IPRs or not should be well informed and take into account both the potential cost and the potential benefits of using formal protection methods. Small business owners, for example, who prefer complexity of product design as a protection method for patentable inventions should be aware that legal action against imitators will be more difficult if their protection efforts prove unsuccessful. The policy implication here is that small firm innovators should always be familiar with the constraints and opportunities of the IPR system.

The fact that numerous small firms do not use IPRs because they attach little importance to innovation protection as a whole also deserves attention. As our results imply, only when a certain degree of innovativeness has been reached do small firm innovators become concerned about the appropriation of innovation results and consequently perceive the use of formal or informal protection methods to be important. Thus, notwithstanding that we have found the non-protection mode to constitute an effective appropriation strategy under certain circumstances, policy-makers should first of all allocate their resources to promoting general innovative activities in small firms, especially those targeted at developing product innovations. Such an approach may necessarily result in a growing use of IPRs by small firms (indeed, also of informal protection methods).

One limitation of our study is the lack of data on alternative means of protection which are particularly relevant to small firms in forming a successful appropriation strategy. Future research efforts should therefore more explicitly consider the role of knowledge tacitness, since we expect this protection mechanism to work more effectively for small rather than large firms. In this regard, although companies with a strong tacit knowledge base, for example, also tend to opt for secrecy protection (see González-Álvarez and Nieto-Antolín, 2007), the 
human resource management of innovative small firms, in particular, is worth being investigated in the context of appropriability. This is because the hiring away of key employees is often the only way for imitators to replicate a company's knowledge assets with a strong tacit component. Furthermore, from this study we see that additional insights are required into the role that complementary assets play in the appropriation of innovation returns in small firms. Finally, our study has not made a direct comparison of the appropriation strategies between small and large firms. An in-depth exploration of potential differences in this regard might be a challenge for future research.

\section{Appendix A}

\section{Table A1}

Description of variables used for validating and profiling the cluster solution; reference period: 2002-2004

\begin{tabular}{|c|c|}
\hline Variable & Description \\
\hline \multicolumn{2}{|l|}{ Variables for validating the cluster solution } \\
\hline Permanent in-house R\&D & 1 if firm conducted in-house $R \& D$ on a permanent basis, 0 otherwise \\
\hline Cooperative innovation & $\begin{array}{l}1 \text { if firm was engaged in innovation-related cooperation with other } \\
\text { firms or scientific institutions, } 0 \text { otherwise }\end{array}$ \\
\hline \multirow{4}{*}{$\begin{array}{l}\text { Total innovation expenditure } \\
\text { (\% of turnover) }\end{array}$} & 1 if firm's intensity of total innovation expenditure is: $0 \% \leq \mathrm{x}<1 \%$ \\
\hline & 2 if firm's intensity of total innovation expenditure is: $1 \% \leq \mathrm{x}<5 \%$ \\
\hline & 3 if firm's intensity of total innovation expenditure is: $5 \% \leq \mathrm{x}<15 \%$ \\
\hline & 4 if firm's intensity of total innovation expenditure is: $x \geq 15 \%$ \\
\hline \multicolumn{2}{|l|}{ Variables for profiling the cluster solution } \\
\hline \multirow[t]{4}{*}{ Industry sector } & $\begin{array}{l}1 \text { if firm's industry is classified as knowledge-intensive manu- } \\
\text { facturing }\end{array}$ \\
\hline & 2 if firm's industry is classified as other manufacturing \\
\hline & 3 if firm's industry is classified as knowledge-intensive services \\
\hline & 4 if firm's industry is classified as other services \\
\hline \multirow[t]{4}{*}{ Type of innovator } & 1 if firm was only active in product innovations, 0 otherwise \\
\hline & 2 if firm was only active in process innovations, 0 otherwise \\
\hline & 3 if firm was active in product and process innovations, 0 otherwise \\
\hline & $\begin{array}{l}4 \text { if firm reports only innovation projects not yet completed (in the last } \\
3 \text { years), } 0 \text { otherwise }\end{array}$ \\
\hline Introduction of new-to-market innovations & $\begin{array}{l}1 \text { if firm introduced product innovations that had not yet been } \\
\text { supplied to the respective market segment, } 0 \text { otherwise }\end{array}$ \\
\hline Introduction of new-to-firm innovations & $\begin{array}{l}1 \text { if firm introduced product innovations that had no predecessors in } \\
\text { the firm's range of products and services, } 0 \text { otherwise }\end{array}$ \\
\hline
\end{tabular}


Table A1 continued

\begin{tabular}{ll}
\hline Variable & Description \\
\hline Introduction of efficiency innovations & $\begin{array}{l}1 \text { if firm introduced process innovations that led to a decrease in unit } \\
\text { costs of production, } 0 \text { otherwise }\end{array}$ \\
Introduction of quality innovations & $\begin{array}{l}1 \text { if firm introduced process innovations that led to an increase in the } \\
\text { quality of products or services, } 0 \text { otherwise }\end{array}$
\end{tabular}

Increased range of goods/services

Entering of new markets

Increased market share

Improved quality in goods/services

Improved flexibility of production/service provision

Increased capacity of production/service provision

Reduced labour costs per unit output

Reduced materials and energy per unit output

Export market activities

Regional business focus

Short product life cycle

Fast pace of technology

Difficult substitution

More than 5 main competitors

Main competitors are larger in size

Price

Quality

Technological edge

Customer service and flexibility
1 if firm considered the impact of this innovation effect to be "high" or "moderate" on a 4-point Likert scale, 0 otherwise

1 if firm considered the impact of this innovation effect to be "high" or "moderate" on a 4-point Likert scale, 0 otherwise

1 if firm considered the impact of this innovation effect to be "high" or "moderate" on a 4-point Likert scale, 0 otherwise

1 if firm considered the impact of this innovation effect to be "high" or "moderate" on a 4-point Likert scale, 0 otherwise

1 if firm considered the impact of this innovation effect to be "high" or "moderate" on a 4-point Likert scale, 0 otherwise

1 if firm considered the impact of this innovation effect to be "high" or "moderate" on a 4-point Likert scale, 0 otherwise

1 if firm considered the impact of this innovation effect to be "high" or "moderate" on a 4-point Likert scale, 0 otherwise

1 if firm considered the impact of this innovation effect to be "high" or "moderate" on a 4-point Likert scale, 0 otherwise

1 if firm had any exports, 0 otherwise

1 if firm only served local or regional markets within a range of about $50 \mathrm{~km}, 0$ otherwise

1 if firm "strongly agrees" or "agrees” on a 4-point Likert scale that products or services mature rapidly in its main market, 0 otherwise

1 if firm "strongly agrees" or "agrees" on a 4-point Likert scale that technologies change rapidly in its main market, 0 otherwise

1 if firm "strongly disagrees" or "disagrees" on a 4-point Likert scale that its products are easily substituted by those of competitors in its main market, 0 otherwise

1 if firm has more than 5 main competitors, 0 otherwise

1 if firm's main competitors are larger in size, 0 otherwise

1 if firm considers price to be an important factor of competition in its main market, 0 otherwise

1 if firm considers quality to be an important factor of competition in its main market, 0 otherwise

1 if firm considers technological edge to be an important factor of competition in its main market, 0 otherwise

1 if firm considers customer service and flexibility to be an important factor of competition in its main market, 0 otherwise 


\section{Table A2}

Factor analysis of the importance of protection mechanisms (principal component factoring, varimax rotated factor loadings)

\begin{tabular}{lccc}
\hline & Factor 1 & Factor 2 & Factor 3 \\
\hline Patent & 0.256 & 0.762 & -0.001 \\
Utility model & 0.085 & 0.797 & 0.118 \\
Industrial design & -0.123 & 0.276 & 0.707 \\
Trademark & 0.198 & 0.446 & 0.448 \\
Copyright & 0.177 & -0.068 & 0.821 \\
Secrecy & 0.774 & 0.329 & -0.007 \\
Complexity of design & 0.821 & -0.088 & 0.142 \\
Lead time advantage & 0.823 & 0.224 & 0.034 \\
\hline Interpretation: & Informal methods & Technical IPRs & Other IPRs \\
\hline Variance explained: & $34.6 \%$ & $17.1 \%$ & $13.0 \%$ \\
\hline
\end{tabular}

\section{References}

Amara, N., Landry, R., Traoré, N., 2008. Managing the protection of innovations in knowledge-intensive business services. Research Policy 37 (9), 1530-1547.

Appiah-Adu, K., Singh, S., 1998. Customer orientation and performance: a study of SMEs. Management Decision 36 (6), 385-394.

Arundel, A., Kabla, I., 1998. What percentage of innovations are patented? Empirical estimates for European firms. Research Policy 27, 127-141.

Arundel, A., 2000. Patents - the viagra of innovation policy?, internal report for the project "Innovation Policy in a Knowledge-Based Economy" commissioned by the European Commission. Retrieved from http://www.edis.sk/ekes/patents.pdf (Accessed June 6, 2010).

Arundel, A., 2001. The relative effectiveness of patents and secrecy for appropriation. Research Policy 30 (4), 611-624.

Aschhoff, B., Blind, K., Ebersberger, B., Fraaß, B., Rammer, C., Schmidt, T., 2007. Schwerpunktbericht zur Innovationserhebung 2005. Bericht an das Bundesministerium für Bildung und Forschung (BMBF). ZEW-Dokumentation Nr. 07-03. Mannheim.

Baldwin, J., Johnson, J., 1996. Business strategies in more- and less-innovative firms in Canada. Research policy 25 (5), 785-804.

Baldwin, J., Gellatly, G., 2003. Innovation strategies and performance in small firms. Edward Elgar, Cheltenham, UK.

Bosworth, D., Webster, E., 2006. An economic perspective. In: Bosworth, D. ,Webster, E. (Eds.), The management of intellectual property. Edward Elgar, Cheltenham, UK, pp. 85-107.

Cohen, W. M., Levin, R. C., 1989. Empirical studies of innovation and market structure. In: Schmalensee, R., Willigand, R. D. (Eds.), Handbook of industrial organization, Volume 2. North-Holland, Amsterdam, pp. 1059-1107. 
Cohen, W. M., Nelson, R. R., Walsh, J. P., 2000. Protecting their intellectual assets: appropriability conditions and why U.S. manufacturing firms patent (or not). NBER Working Papers Series (7552).

Curran, J., Blackburn, R. A., 2001. Researching the small enterprise. SAGE Publications, London.

Dosi, G., Marengo, L., Pasquali, C., 2006. How much should society fuel the greed of innovators? On the relations between appropriability, opportunities and rates of innovation. Research Policy 35 (8), 1110-1121.

European Commission, 2006. Putting knowledge into practice: A broad-based innovation strategy for the EU - Communication from the Commission to the Council, The European Parliament, The European Economic and Social Committee, and the Committee of Regions, COM (2006) 502, Brussels, Belgium. Retrieved from http://eurlex.europa.eu/LexUriServ/site/en/com/2006/com2006_0502en01.pdf (Accessed June 7, 2010).

Evangelista, R., 2000. Sectoral patterns of technological change in services. Economics of innovation and new technology 9 (3), 183-221.

González-Álvarez, N., Nieto-Antolín, M., 2007. Appropriability of innovation results: an empirical study in spanish manufacturing firms. Technovation 27 (5), 280-295.

Gopalakrishnan, S., Bierly, P., Kessler, E. H., 1999. A reexamination of product and process innovations using a knowledge-based view. The journal of high technology management research $10(1), 147-166$.

Granstrand, O., 1999. The economics and management of intellectual property: towards intellectual capitalism. Edward Elgar, Cheltenham, UK.

Greenhalgh, C., Rogers, M., 2007. The value of intellectual property rights to firms and society. Oxford Review of Economic Policy 23 (4), 541-567.

Grupp, H., Legler, H., Jungmittag, A., Schmoch, U., 2000. Hochtechnologie 2000 Neudefinition der Hochtechnologie für die Berichterstattung zur technologischen Leistungsfähigkeit Deutschlands. Karlsruhe/Hannover.

Guellec, D., Van Pottelsberghe de la Potterie, B., 2007. The economics of the European patent system. IP policy for innovation and competition. Oxford University Press, Oxford.

Hair, J. F., Anderson, R. E., Tatham, R. L., Black, W. C., 1998. Multivariate Data Analysis. fifth ed. Prentice Hall, Upper Saddle River, NJ.

Hall, B. H., Ziedonis, R. H., 2001. The patent paradox revisited: An empirical study of patenting in the US semiconductor industry, 1979-1995. The Rand journal of economics 32 (1), 101-128.

Hall, M., Oppenheim, C., Sheen, M., 2003. Barriers to the use of patent information in SMEs. In: Blackburn, R. A. (Eds.), Intellectual property and innovation management in small firms. Routledge, London, New York, pp. 144-160.

Hanel, P., 2008. The use of intellectual property rights and innovation by manufacturing firms in Canada. Economics of Innovation and New Technology 17 (3), 285-309.

Harabi, N., 1995. Appropriability of technical innovations. An empirical analysis. Research Policy 24 (6), 981-992. 
Hollenstein, H., 2003. Innovation modes in the Swiss service sector: a cluster analysis based on firm-level data. Research Policy 32 (5), 845-864.

Hurmelinna-Laukkanen, P., Puumalainen, K., 2007. Nature and dynamics of appropriability: strategies for appropriating returns on innovation. R\&D Management 37 (2), 95-112.

Hurmelinna-Laukkanen, P., Sainio, L.-M., Jauhiainen, T., 2008. Appropriability regime for radical and incremental innovations. R\&D Management 38 (3), 278-289.

Hurmelinna-Laukkanen, P., 2009. The availability, strength and efficiency of appropriability mechanisms - protecting investments in knowledge creation. International Journal of Technology Management 45 (3), 282-290.

Hurmelinna, P., Kyläheiko, K., Jauhiainen, T., 2007. The Janus face of the appropriability regime in the protection of innovations: Theoretical re-appraisal and empirical analysis. Technovation 27 (3), 133-144.

Hussinger, K., 2004. Is silence golden? Patents versus secrecy at the firm level. Centre for European Economic Research (ZEW), Discussion Paper 04-78.

Iversen, E. J., 2003. Norwegian small and medium-sized enterprises and the intellectual property rights system: exploration and analysis, a study carried out for the World Intellectual Property Organization (WIPO), Retrieved from http://www.wipo.int/sme/en/documents/research/norway/norway_study.pdf (Accessed June 4, 2010). Oslo.

Iversen, E. J., 2008. IPRs and Norwegian enterprises: diversification of innovative efforts in Norwegian firms. In: Carayannis, E. G., Kaloudis, A., Mariussen, A. (Eds.), Diversity in the knowledge economy and society: heterogeneity, innovation and entrepreneurship. Edward Elgar, Cheltenham, UK, pp. 191-216.

Jantunen, A., Hurmelinna-Laukkanen, P., 2006. Entrepreneurial orientation, appropriability regimes and innovation performance. International Journal of Learning and Intellectual Capital 3 (2), 153-166.

Janz, N., Ebling, G., Gottschalk, S., Niggemann, H., 2001. The Mannheim innovation panels (MIP and MIP-S) of the Centre for European Economic Research (ZEW). Schmollers Jahrbuch - Zeitschrift für Wirtschafts- und Sozialwissenschaften 121, 123-129.

Jennewein, K., 2005. Intellectual property management: the role of technology-brands in the appropriation of technological innovation. Physica-Verlag, Heidelberg.

Jensen, M.-B., Johnson, B., Lorenz, E., Lundvall, B.-Å., 2007. Forms of knowledge and modes of innovation. Research Policy 36 (5), 680-693.

Jensen, P. H., Webster, E., 2006. Firm size and the use of intellectual property rights. The Economic Record 82 (256), 44-55.

de Jong, J., Marsili, O., 2006. The fruit flies of innovations: A taxonomy of innovative small firms. Research Policy 35 (2), 213-229.

Kitching, J., Blackburn, R. A., 1998. Intellectual property management in the small and medium enterprise (SME). Journal of Small Business and Enterprise Development 5 (4), 327-335.

Kitching, J., Blackburn, R. A., 2003. Innovation, intellectual property and informality: evidence from a study of small enterprises and some implications for policy. In: Blackburn, R. A. (Eds.), Intellectual property and innovation management in small firms. Routledge, London, New York, pp. 16-34. 
König, H., Licht, G., 1995. Patents, R\&D and Innovation. Evidence from the Mannheim Innovation Panel. IFO-Studien 41 (4), 521-544.

Lanjouw, J. O., Schankerman, M., 2004. Protecting intellectual property rights: Are small firms handicapped? The Journal of Law \& Economics 47 (1), 45-74.

Laursen, K., Salter, A., 2005. My precious. The role of appropriability strategies in shaping innovative performance. DRUID Working Paper No. 05-02, Danish Research Unit for Industrial Dynamics.

Leiponen, A., Byma, J., 2009. If you cannot block, you better run: Small firms, cooperative innovation, and appropriation strategies. Research Policy 38 (9), 1478-1488.

Levin, R. C., Cohen, W. M., Mowery, D. C., 1985. R\&D appropriability, opportunity, and market structure: New evidence on some Schumpeterian hypothesis. American Economic Review, Papers and Proceedings 75 (2), 20-24.

Levin, R. C., Klevorick, A. K., Nelson, R. R., Winter, S. G., 1987. Appropriating the returns from industrial research and development. Brookings Papers on Economic Activity 3, 783-831.

López, A., 2009. Innovation and appropriability: Empirical evidence and research agenda. In: World Intellectual Property Organization (Eds.), The economics of intellectual property. Suggestions for further research in developing countries and countries with economies in transition. Retrieved from http://www.wipo.int/export/sites/www/ipdevelopment/en/economics/pdf/wo_1012_e_ch_1.pdf (Accessed May 16, 2010), pp. 132.

Macdonald, S., 2004. When means become ends: considering the impact of patent strategy on innovation. Information Economics and Policy 16 (1), 135-158.

Malerba, F., Orsenigo, L., 1993. Technological regimes and firm behavior. Industrial and Corporate Change 2 (1), 45-71.

Malerba, F., Orsenigo, L., 1997. Technological regimes and sectoral patterns of innovative activities. Industrial and Corporate Change 6 (1), 83-117.

Malerba, F., 2002. Sectoral systems of innovation and production. Research Policy 31 (2), 247-264.

Mansfield, E., 1986. Patents and Innovation: An Empirical Study. Management Science 32 (2), 173-181.

Matthews, D., Pickering, J., Kirkland, J., 2003. A strategic approach to managing intellectual property. In: Blackburn, R. A. (Eds.), Intellectual property and innovation management in small firms. Routledge, London, New York, pp. 35-54.

Mazzarol, T., Reboud, S., 2009. The strategy of small firms. Strategic management and innovation in the small firm. Edward Elgar, Cheltenham, UK.

Nieto, M., Pérez-Cano, C., 2004. The influence of knowledge attributes on innovation protection mechanisms. Knowledge and Process Management 11 (2), 117-126.

Nooteboom, B., 1994. Innovation and diffusion in small firms: Theory and evidence. Small Business Economics 6 (5), 327-347.

Päällysaho, S., Kuusisto, J., 2008. Intellectual property protection as a key driver of service innovation: an analysis of innovative KIBS businesses in Finland and the UK. International Journal of Services Technology and Management 9 (3/4): 268-284. 
Pavitt, K., 1984. Sectoral patterns of technical change: towards a taxonomy and a theory. Research Policy 13 (6), 343-373.

Penrose, E. T., 1959. The theory of the growth of the firm. Blackwell, Oxford.

PRO INNO Europe, 2007. A memorandum on removing barriers for a better use of IPR by SMEs, A Report for the Directorate-General for Enterprise and Industry (European Commission) by an IPR Expert Group, Paper No. 3, Retrieved from http://www.proinnoeurope.eu/promotion-pro-inno-europe-results/page/publications (Accessed June 15, 2010).

Radauer, A., Streicher, J., Ohler, F., 2007. Benchmarking National and Regional Support Services for SMEs in the Field of Intellectual and Industrial Property, Final Benchmarking Report on Behalf of the European Commission, Austrian Institute for SME Research. Retrieved from http://www.insme.org/documents/Benchmarking-ReportSME.pdf (Accessed June 2, 2010).

Ramello, G. B., Silva, F., 2006. Appropriating signs and meaning: the elusive economics of trademark. Industrial and Corporate Change 15 (6), 937-963.

Rammer, C., Czarnitzki, D., Spielkamp, A., 2009. Innovation success of non-R\&Dperformers: substituting technology by management in SMEs. Small Business Economics 33 (1), 35-58.

Rothwell, R., 1983. Innovation and firm size: a case for dynamic complementarity; or, is small really so beautiful? Journal of General Management 8 (3), 5-25.

Sattler, H., 2003. Appropriability of product innovations: An empirical analysis for Germany. International Journal of Technology Management 26 (5-6), 502-516.

Scotchmer, S., 2004. Innovation and Incentives. MIT Press, Cambridge, Massachusetts.

Teece, D. J., 1986. Profiting from technological innovation: Implications for integration, collaboration, licensing and public policy. Research Policy 15 (6), 285-305.

Teece, D. J., 2002. Managing intellectual capital. Organizational, strategic, and policy dimensions. Oxford University Press, Oxford.

Teece, D. J., 2003. The strategic management of technology and intellectual property In: Faulkner, D. O., Campbell, A. (Eds.), The Oxford Handbook of Strategy. Volume I: A Strategy Overview and Competitive Strategy. Oxford University Press, Oxford, pp. 132166.

Teece, D. J., 2006. Reflections on “Profiting from Innovation”. Research Policy 35 (8), 1131-1146.

WIPO (2003). Intellectual property (IP) rights and innovation in small and medium-sized enterprises, World Intellectual Property Organization (WIPO), Retrieved from http://www.wipo.int/export/sites/www/sme/en/documents/pdf/iprs_innovation.pdf (Accessed June 2, 2010).

WIPO (2004). WIPO Intellectual Property Handbook: Policy, Law and Use. World Intellectual Property Organization, Publication No.489. Retrieved from http://www.wipo.int/export/sites/www/about-ip/en/iprm/pdf/ch2.pdf (Accessed June 29, 2010).

Wynarczyk, P., Watson, R., Storey, D., Short, H., Keasey, K., 1993. Managerial labour markets in small and medium-sized enterprises. Routledge, London. 


\section{Kapitel IV}

\section{Innovation protection practices in small and medium enterprises (SMEs) ${ }^{1}$}

1 Der vorliegende Einzelbeitrag wurde im Rahmen eines Kooperationsprojekts des Volkswirtschaftlichen Instituts für Mittelstand und Handwerk an der Universität Göttingen (ifh) und der KfW Bankengruppe/Abteilung Volkswirtschaft erstellt. 


\section{Introduction}

The creation of innovative capabilities is seen as a major driver for competitiveness at the national, industrial and company levels (Cantwell 2005). Yet, introducing new products or processes is not enough if individual companies want to remain competitive, since they must also be able to achieve a reasonable rate of return on such activities. Success in appropriation, however, is far from easy because of the public good characteristics of knowledge. As a consequence, companies easily fail to get enough value from their innovations at the comercialization stage, which can severely undermine incentives for further innovation (Arrow 1962; Geroski 1995). Starting from this premise, the ways firms are trying to protect their innovations from imitation in order to secure corresponding returns have been an enduring research theme for some time. Some of the main topics under investigation included the comparative effectiveness of various formal and informal protection mechanisms such as intellectual property rights (IPRs) and lead time or secrecy (e.g. Levin et al. 1987; Harabi 1995; Cohen et al. 2000); the explanatory determinants of their perceived importance in terms of use and effectiveness (e.g. Galende 2006; Amara et al. 2008; Hanel 2008) or, more specifically, the relationships between attributes of knowledge such as tacitness and complexity, and a firm's choice of certain protection mechanisms (e.g. Nieto/Pérez-Cano 2004; GonzálezÁlvarez/Nieto-Antolín 2007; Hurmelinna-Laukkanen/Puumalainen 2007).

Though a much clearer understanding was obtained in these studies of the roles of various mechanisms in the protection of returns on innovation, there are, nevertheless, still many unanswered questions on this subject (see López 2009). One concerns the explanatory determinants of innovation protection practices in small and medium enterprises (SMEs), a topic which is rather limited in the existing literature. Most previous empirical studies address this issue only in discussing the positive effect of the size of a firm on the relevance of patents or other IPRs to appropriation purposes (see Arundel 2001; Sattler 2003; Galende 2006; González-Álvarez/Nieto-Antolín 2007; Amara et al. 2008; Hanel 2008). Broadly speaking, they come to the conclusion that smaller firms are less likely to rely on IPRs, probably because of cost and complexity issues associated with the IPR system, a fact which raises certain implications for innovation policy initiatives that aim to foster the use of IPRs by SMEs.

However, the lesser use of formal protection mechanisms by smaller firms should not be associated merely with size-related disadvantages inherent in the IPR system, as it may also reflect distinguishing features between small and large firms when it comes to appropriation strategies. Indeed, a telephone survey and subsequent face-to-face interviews conducted by Kitching and Blackburn (1998; 2003) indicate that SMEs often prefer informal protection mechanisms (e.g. the creation of high-trust relationships with customers and suppliers or the maintenance of a lead time advantage over competitors) because they find them more familiar, cheaper, less time-consuming and more effective than IPRs. Furthermore, most respondents in this sample of almost 400 SMEs from four sectors in the UK (computer software, design, electronics, mechanical engineering) saw informal protection practices as vital to the commercialization of innovations and hence as a key component of their broader competitive 
strategy. Thus, the majority of SME owners did not consider the access to and use of IPRs an impediment to the successful appropriation of innovation returns, which is why they were largely indifferent to the formal IPR system. Kitching and Blackburn (1998; 2003) thus concluded that policy attempts to remove barriers to IPR usage may have little impact on innovation by SMEs.

Hence, given that successful appropriation strategies in smaller firms are in fact characterized by a higher degree of informality than those in larger firms, the explanatory determinants of the perceived importance of formal and in particular of informal protection mechanisms should also be examined from an SME perspective. To our knowledge, the only study in this regard is that of Leiponen and Byma (2009). Based on a survey of 504 small knowledgeintensive Finnish firms in the manufacturing and service industries, this study aims to explain the relative importance of three innovation protection mechanisms (patents vs. secrecy vs. speed to market). It is again shown that the majority of small firms prefer informal innovation protection methods to IPRs. Only highly R\&D-intensive small firms and those that cooperate in R\&D with universities saw patents as the most important appropriation instrument. Indeed, it became evident that innovation-related cooperation activities in general have a major impact on the kind of protection mechanism chosen by SMEs. The evidence given by Leiponen and Byma (2009) further indicates that small firms tend for the most part to benefit from a speed to market strategy in their efforts to achieve a lead time advantage over competitors. Given their results, the authors argue that appropriation strategies pursued by small firms differ qualitatively from those of large firms. Moreover, they expect smaller firms to be disadvantaged by their size in the use of IPRs and suggest a critical re-evaluation of current patentfocused IPR policies.

Against this background, the contribution of the present paper is twofold: Firstly, our approach resembles that of a baseline study in this field of research. The empirical analysis is based on a large data set of innovative SMEs from all sectors of the German economy, allowing a number of hypotheses about the perceived importance of various formal and informal protection mechanisms to be tested. In this way, we aim to account for the very heterogeneous nature of SMEs, shown, for example, by the fact that, although smaller firms are less likely to make use of IPR protection on average, there is evidence that IPR-active SMEs can use patents or other IPRs more intensively than larger firms (see Brouwer/Kleinknecht 1999; Greenhalgh/Rogers 2007). Secondly, several hypotheses in Section 2 are proposed from a knowledge-based view, since much of the operating knowledge in innovative SMEs tends to be tacit (Nooteboom 1994). A key reason for this is that smaller firms, as a result of their scalerelated disadvantages, either generally place less emphasis on Research \& Development (R\&D) in achieving innovative results (e.g. by instead relying on "soft" capabilities such as the skills of their staff or collaborative activities with customers), or, often, perform in-house R\&D in a more informal and occasional manner than larger firms (Rothwell 1983; Kleinknecht 1987; 1991; Baldwin/Gellatly 2003; Rammer et al. 2009). In reference to the two ideal modes of learning and innovation contrasted by Jensen et al. (2007), innovation in SMEs visà-vis that in larger firms should therefore be relatively more based on informal processes of 
learning and experience-based know-how with strong tacit elements than on formal processes of $R \& D$ related to the production and use of codified scientific and technical knowledge. Thus, as patents, for example, are by definition a codification of inventions (see Section 2), informal innovation protection practices may in fact work more effectively for a large number of innovative SMEs because they correspond better to their specific mode of innovation. However, this is not to say that IPR protection cannot be of primary importance for particular subgroups of innovative SMEs, as the great diversity among smaller firms should also be of relevance in this knowledge-related case. In summing up, particularly in regard to SMEs we thus expect a knowledge-based view to be a fruitful approach for explaining different forms of innovation protection practices.

The rest of this paper is organised as follows: Section 2 derives empirical hypotheses about the importance as perceived by SMEs of formal and informal protection mechanisms in the light of the existing literature (more precisely: patents, other IPRs, secrecy, complexity of design, long-term retention of qualified personnel and lead time advantage). Our data set and descriptive results are presented in Section 3. Section 4 summarises and discusses the estimation results. Concluding remarks and implications for policy and research are provided in Section 5.

\section{Literature and empirical hypotheses}

Various characteristics of innovative SMEs should explain their perception of the importance of the formal and informal protection mechanisms under consideration. To begin with, and regarding the above discussion, the existence of some form of in-house $R \& D$ should play a role, because such a capacity represents one important source for the internal generation of new knowledge and has a positive impact on the innovative performance of SMEs (see Bougrain/Haudeville 2002; Hall et al. 2009; Rammer et al. 2009). Hence, the risk of imitation and therefore the overall need for innovation protection practices should naturally increase as a result of such activities. Yet, the conduct of in-house R\&D on a permanent basis should especially encourage the choice of patents as the preferred appropriation method, since more intense R\&D-activities tend to be associated with higher degrees of formality and knowledge codification (Brusoni et al. 2005; Jensen et al. 2007). In fact, the ability to describe the originality of an invention by adopting a techno-scientific language is a necessary precondition for a successful patent application, which is why an innovator's preference for patent protecttion increases with the codifiability of the embodied technology (Nieto/Pérez-Cano 2004; González-Álvarez/Nieto-Antolín 2007). Furthermore, as Arundel (2001) points out, innovations developed by continuously R\&D-performing firms are more likely to be in line with the novelty requirement for granting a patent. This leads us to formulate two hypotheses:

H1.1: Conducting some form of in-house $R \& D$ increases the overall need for innovation protection practices.

H1.2: Permanent in-house R\&D increases the preference for patent protection. 
While codified knowledge is relatively easy to communicate and transfer, the opposite holds true for tacit knowledge, owing to its largely person-embodied nature. Because of this, innovative results with a strong tacit knowledge component are less prone to be imitated and can thus be a key factor in a firm's establishing and sustaining a competitive advantage (Reed/DeFillippi 1990; Zander/Kogut 1995). For this reason, tacitness in itself can even be seen as a separate means of appropriation (Hurmelinna-Laukkanen/Puumalainen 2007; HurmelinnaLaukkanen 2009). In fact, by virtue of their strong tacit knowledge base, this appropriation method works particularly effectively for smaller firms (Nooteboom 1994). However, such beneficial effects diminish in the case of the hiring away of key staff by competitors (Teece 2003). For a firm to secure its tacit knowledge against imitation it is therefore often important to employ further methods rather than relying solely on limited knowledge transferability, which is why we expect the long-term retention of qualified personnel to be of particular relevance when it comes to the protection of tacit elements. Yet, despite its widely recognized importance, "tacit knowledge remains a very nebulous concept and is extremely difficult to measure and evaluate" (Howells 1996: 94). To be more clear in this regard we propose the concept of 'craftsmanship', since tacitness lies at the heart of craft knowledge (Sennett 2008). Indeed, apprenticeship relations, where the apprentice is able to develop certain skills only through a process of learning-by-doing under the guidance of a master craftsman are a typical example of the build-up of tacit know-how (Polanyi 1958/1974; see also Gamble 2001; 2004). Therefore, the existence of craft skills can serve as one possible indicator of the capture of tacit knowledge elements in innovative SMEs (regarding this argument see also Nooteboom 1994 and Rantakyrö 2005). The corresponding hypothesis can thus be stated more precisely:

H2: Craft knowledge is effectively protected by the long-term retention of qualified personnel.

The type of an innovation (i.e. product or process) might also have an impact on the relevance of certain protection mechanisms for various knowledge-related reasons. To begin with, process innovations are less observable to potential imitators than new products that are launched on the market, which is why process technology is less likely to be the subject of unwanted reverse engineering. So, in the case of process innovation the use of secrecy in protecting against imitation is often a viable alternative to a reliance on patents with their disclosure requirements (Teece 2003; Nieto/Pérez-Cano 2004). In addition, new knowledge generated through process innovation tends to be more complex than that associated with innovations of products. It probably also incorporates a stronger tacit component, since it often results from experience-based know-how and an intimate familiarity with the processes involved (Gopalakrishnan et al. 1999). Hence, patent protection may also be deemed less important by process innovators, owing to a higher effectiveness of the complexity of design and the long-term retention of qualified personnel in preventing imitation by competitors. The corresponding hypotheses are as follows:

H3.1: Process innovators are less likely to regard patents as an important protection mechanism. 
H3.2: Process innovations are effectively protected by secrecy, the complexity of design and the long-term retention of qualified personnel.

Furthermore, we also expect the engagement of SMEs in cooperating with external partners to play a key role in this. On the one hand, by the possibility of using additional resources, collaboration with other companies (i.e. competitors, suppliers or clients) or with scientific institutions (i.e. universities or public research establishments) can enable firms to innovate continuously and hence more quickly than their rivals, which will often result in a lead time advantage over potential imitators (Galende 2006; Beyer/Stephan 2008). Lead time may therefore be an effective means of appropriation for cooperating SMEs, since smaller firms often depend on such external relationships to compensate for their resource disadvantages in innovation (Hadjimanolis 2000; Hewitt-Dundas 2006). On the other hand, cooperation activities are also expected to be significant, because collaborating firms always have to find a balance between the benefits of knowledge sharing and the simultaneous need for protection against the critical loss of firm-specific knowledge (Norman 2002; Hurmelinna et al. 2007). This should be especially true for the protection of tacit knowledge since the bringing together of corresponding resources often serves as a key element in cooperation activities between firms or between firms and scientific institutions. The only way to increase competitiveness in this manner, however, is by the transfer and sharing of tacit know-how through staff links or flows between collaborating partners (Howells 1996; Norman 2002). Hence, effective efforts to maintain staff loyalty and thus protect tacit knowledge from leaking out should be of higher relevance for cooperating SMEs. In this regard, in the following two ways cooperation agreements with scientific institutions may encourage the long-term retention of qualified personnel by innovative SMEs in particular. Firstly, employing qualified individuals who are able to assimilate and exploit the scientific and technological knowledge generated by universities or public research establishments can be seen as a prerequisite for firms to be successfully engaged in such activities ('absorptive capacity', see Cohen/Levinthal 1989; 1990). Hence, since smaller firms tend to lack suitably qualified manpower in innovation activities vis-à-vis larger firms (Rothwell 1983), innovative SMEs which cooperate with scientific institutions may undertake special efforts to protect such internal resources. Secondly, by the same argument, the possibility of recruiting trained scientists and engineers (university employees, graduates or doctoral students) as a typical outcome of scientific cooperation may further increase the importance of staff-related appropriation strategies for these firms. However, despite the fact that personnel recruitment is an important channel for the flow of tacit knowledge from scientific institutions to private firms, the more codified results of such interactions become visible, for example, through the co-generation of patents by cooperating partners (Schartinger et al. 2002; Bekkers 2008; Levy et al. 2009). Therefore, innovative SMEs cooperating with scientific institutions may also attribute higher relevance to patent protection. To sum up, the following hypotheses can be posed:

H4.1: Cooperation with external partners increases the importance of lead time as a protection mechanism. 
H4.2: Cooperation with external partners, particularly with scientific institutions, increases the importance of the long-term retention of qualified personnel.

H4.3: Cooperation with scientific institutions increases the importance of patent protection.

With regard to the overall relevance of IPRs to innovation protection, it should matter if an innovative SME has to compete against larger counterparts. Large firms are much more able than small ones to make extensive use of IPRs, owing to their lower cost constraints. As a result of their specialized in-house intellectual property expertise (e.g. their own patent departments), they are also more likely to deal with the IPR system in a professional and strategic manner (Matthews et al. 2003; Blind et al. 2006; Radauer et al. 2007). Furthermore, from a knowledge-based view it could be argued that the knowledge developed in the formal R\&D departments of large firms tends to be more easily protected by patents or other IPRs because of its explicit and codified nature (Jensen et al. 2007). In order to reduce the likelihood that large firms use this capacity to gain a competitive advantage, SME innovators probably need a higher awareness of the IPR system and should rely more strongly on corresponding legal protection methods when their main competitors are relatively large. Otherwise large firms may, for example, find themselves in a better position to sue SMEs for infringing on their IPRs, to replicate SMEs' inventions through reverse engineering without legal sanction and subsequently even to file for their own IPRs. On the other hand, smaller firms face strong difficulties in enforcing their IPRs successfully when it comes to litigation against larger competitors (Kingston 2000; Lanjouw/Schankerman 2004). There is anecdotal evidence that at least some SME owners forgo potential patent applications to avoid intimidation by large firms with their greater resource base for litigation, and instead rely on secrecy protection or a lead time advantage (see Hall et al. 2003; Kitching/Blackburn 2003; Macdonald/Lefang 2003). Hence, to gain further insight into this competitive interaction between SMEs and large firms we propose the following hypotheses:

H5.1: SMEs competing against large firms attribute higher importance to IPR protection.

H5.2: SMEs competing against large firms attribute higher importance to secrecy and lead time.

The engagement of an innovative SME in export markets should also have an impact on its conditions of appropriability. Patents can be an important precondition for firms to enter foreign markets successfully (Arundel 2000; Blind et al. 2006). Hence, exporting firms may find IPR protection more important than other forms, especially that of patents. Yet, as De Faria and Sofka (2010) have shown, export activities can increase the relevance of formal as well as informal innovation protection mechanisms. According to the authors, however, the explanation for this result is not straightforward. Following their line of argument, on the one hand, exporting SMEs may be more innovative in the first place (for this argument see Baldwin and Johnson 1996), while on the other, export market activities may serve as a proxy for the intensity of competition that a firm is facing (for this argument see Arundel and Kabla 1998). Even though the direction of causality is not clear in this regard, a higher risk of imita- 
tion may be a likely result either way. We therefore expect innovative SMEs with export activity to be more dependent than others on a profound appropriation strategy. This is expressed through the following hypothesis:

H6: Exporting SMEs attribute a higher overall importance to the use of innovation protection mechanisms.

In addition, the industry sector in which an innovative SME is operating should be of relevance. Patterns of innovation in the service sector differ in certain ways from those in manufacturing, which has implications for the shape of appropriability conditions in corresponding industries. A distinctive feature of many service products is their intangible nature, making them harder to store, transport or export and, last but not least, to protect within the formal IPR system than is the case with typical manufacturing products (Blind et al. 2003; Miles 2005). As the results of Tether (2005) and Leiponen (2005) indicate, one important reason for this is the fact that innovation in services is based less on technological advances and formal R\&D activities. Instead, compared with manufacturing firms, many service firms tend to place greater emphasis on the professional skills of their employees and collaborative interactions with their clients and suppliers to find innovative solutions. Service innovations therefore often demand a more informal approach to the management and organization of the innovation process, which is why Päällysaho and Kuusisto (2008) expect informal protection mechanisms to be particularly effective in the context of services. First of all, this should be the case for the long-term retention of qualified personnel, since the emphasis of service firms on human resources as the key internal source of innovation may be naturally reflected in a higher relevance of maintaining staff loyalty to protect innovative results from imitation. Moreover, the prominent role of tacit knowledge in the close interactions which many innovative service firms maintain with their clients and suppliers should further increase the importance of staff-related appropriation strategies in this regard. In sum, we therefore hypothesize:

H7.1: Service SMEs attribute lower importance to IPR protection than manufacturing SMEs.

H7.2: Service SMEs attribute higher importance to informal protection mechanisms than do manufacturing SMEs, in particular to the long-term retention of qualified personnel.

When it comes to the role of patents in innovation protection, the size of a firm may also be an explanatory factor amongst innovative SMEs. As previous studies have shown (see Section 1), larger firms are, on average, more likely to rely on patents as a means of achieving appropriability. Possible reasons for this, as argued by Arundel (2001), are that many smaller firms cannot afford the costs of patent application, or lack the resources necessary to enforce their rights in the case of infringement. Their innovations are also often based on minor incremental improvements which are not patentable. As a result, small firms may, for example, switch to secrecy as a low-cost alternative (Arundel 2001; Burr et al. 2007) or they may use complex product design as a technology-based substitute for patent protection (Amara et al. 2008; Päällysaho/Kuusisto 2008). We extend these arguments to our discussion of innovation 
protection practices in SMEs and expect a positive relationship between the size of a firm (as measured by the number of its employees) and the use of patents. Yet, the results of Hanel (2008) and Amara et al. (2008) indicate that the perceived importance of other IPRs such as trademarks or copyrights may also increase with the size of a firm, which is why we propose our hypothesis with regard to IPR protection in general. Furthermore, the positive effect of company size may also hold for the use of lead time, since the ability to innovate continuously over time and thus sustain a lead time advantage over competitors often requires constant investment of resources, especially into human capital in terms of skills and motivation of the work force (Galende 2006; González-Álvarez/Nieto-Antolín 2007). Hence, larger SMEs may be more able to employ a lead time strategy to appropriate their innovative results, as they probably lack suitably qualified personnel to a lesser degree than smaller SMEs. Thus, we can derive two hypotheses:

H8.1: Larger SMEs are more likely to rely on IPR protection than smaller ones.

H8.2: Larger SMEs are more likely to rely on lead time than smaller ones.

Finally, the age of a company may be significant. This is due to the fact that the intensity of innovation activities during the reference period should be linked to the time that has elapsed since an innovative SME entered the market. Huergo and Jaumandreu (2004) have shown that small entrant firms tend to present a higher propensity to innovation than older firms, which points to the increased importance of technology entrepreneurship over the past decades. Similarly, the results of Rammer et al. (2009) indicate that younger SMEs tend to be more successful in innovation than older ones. In this case, innovation success was measured through the number of different types of product and process innovations that a firm had introduced during the reference year. Given that the application of an effective appropriation strategy in profiting sufficiently from one's own innovations is therefore a key management challenge especially for start-up firms (Gans et al. 2002; Gans/Stern 2003), younger SMEs may also display a higher probability of employing formal and informal innovation protection mechanisms. The following hypothesis reflects this idea:

H9: Younger SMEs attribute a higher overall importance to the use of innovation protection mechanisms.

\section{Data set and descriptive statistics}

The empirical analysis rests on data from the 2007 survey wave by the KfW-Mittelstandspanel, a panel survey of German SMEs conducted by the KfW Bankengruppe, Frankfurt/Main on an annual basis. The corresponding target population comprises all small and medium enterprises of the German economy with an annual turnover of up to 500 million euros. A distinct advantage of the survey over comparable datasets (e.g. the German contribution to the Community Innovation Surveys) is its broad size coverage, since firms with fewer than five employees in particular are included in the survey. Based on a broad set of questions (e.g. on business development, investment, or finance), the KfW-Mittelstandspanel offers detailed in- 
formation on SMEs, allowing in-depth analysis of their economic behaviour (e.g. see Müller/Zimmermann 2009; Zimmermann 2009). In addition, information is collected in individual waves on special topics of interest. For example, in 2007 several questions on various fields of innovation were included. Of particular interest in this regard are respondents' assessments of different formal and informal mechanisms in terms of their relevance for protecting companies' own innovations against unwanted imitation.

The population of the 2007 survey wave (reference period 2004-2006) was drawn as a stratified random sample. The variables used for stratification were firm size (six size classes, i.e. up to 4 employees, 5-9, 10-19, 20-49, 50-99 and 100 or more employees), industry classification (manufacturing, construction, retail, wholesale, and services), region (East or West Germany) and participation in a government support programme for SMEs conducted by the KfW Bankengruppe. The gross sample comprised a total of 73,917 firms, 13,328 of which returned a questionnaire (Reize 2007). This represents a response rate of $18 \%$, which lies in the typical range for this kind of survey. For the further analysis we used information on 4,162 companies, since we restricted our data set in two ways. Firstly, it was confined to the subsample of innovative companies, which was straightforward since we are interested in strategies taken by SME innovators to keep imitators at bay. However, in this way we also aim to avoid potential reverse causality in the later regression analysis between the decision to innovate in the first place and the essential ability of firms to appropriate sufficient profits from their own innovations and remain competitive (on the usefulness of this distinction see Teece 2006). In accordance with the Oslo Manual guidelines (OECD/Eurostat 2005), a company is classified as innovative if it has introduced new or significantly improved goods or services (product innovation) and/or if it has implemented new or significantly improved production or delivery methods (process innovation) during the reference period. Secondly, from the perspective of our study, firms with more than 499 full-time employees are removed from the dataset to be sure that we keep our focus on smaller-sized firms.

As already noted, the dependent variables of the paper are based on a question which asked respondents to rate on a binary scale (“important” or "not important”) several innovation protection mechanisms with regard to their firm-specific relevance. The first set of mechanisms makes reference to the formal IPR system. In this case we are able to make a distinction between the protection of innovation returns via patents and that via other IPRs (i.e. utility models, trademarks and copyrights grouped under one heading). The second set of mechanisms comprises as informal means of protection secrecy, long-term retention of qualified personnel and lead time advantage. Descriptive statistics for the corresponding variables are provided in Table 1. Overall, informal protection practices are reported to be important more frequently than IPRs. Lead time is the most commonly used mechanism (59.39\% of innovative SMEs) followed by the long-term retention of qualified personnel, secrecy and complexity of design. However, sizeable shares of SME innovators also deem patents (23.57\%) and other IPRs (21.77\%) to be important for protection purposes. Hence, as expected, informal innovation protection methods seem to be of particular relevance for a large number of SMEs. However, 
the descriptive results also indicate that IPRs can be an important means of protection in the SME sector.

Table 1 Perceived importance of innovation protection mechanisms ( 1 = “important"; $0=$ “not important”)

\begin{tabular}{lc}
\hline & in \% of innovative SMEs (N = 4162) \\
\hline Patents & 23.57 \\
Other IPRs (utility models, trademarks, copyrights) & 21.77 \\
Secrecy & 29.05 \\
Complexity of design & 18.02 \\
Long-term retention of qualified personnel & 41.98 \\
Lead time advantage & 59.39 \\
\hline
\end{tabular}

Note: Percentages do not add up to $100 \%$ as multiple answers were allowed.

Descriptions of the independent and the control variables used in the explanatory analysis (Section 4) are shown in Table A1 (see Appendix). As a main explanatory variable set of interest, the existence and continuity of R\&D-activities are measured through three binary variables. The descriptive statistics show that although the majority of SME innovators refrained from conducting their own R\&D activities during the reference period, a sizeable share of innovative SMEs did conduct in-house R\&D occasionally (16.63\%) or even on a permanent basis (21.05\%). The binary variable 'craft skills' is created by using respondents' answers to whether their main firm-internal activities are craft-based or not. In this regard, it has to be noted that the German craft sector is not defined by numbers of employees or financial turnover but instead by law, as including a large number of craft and craft-related occupations in areas such as trades for industrial needs, construction trades, automobile trades and health trades, implying that corresponding companies are active in almost all parts of the economy (i.e. in manufacturing as well as in service industries, for example). In our sample, $35.92 \%$ of innovative SMEs considered their main activities to be of a craft-based nature.

To investigate the influence of the type of innovation, a binary variable indicates whether an innovative SME has introduced process innovations in the past three years. In fact this holds true for almost $62 \%$ of the companies in the data set. Inter-firm cooperation and scientific cooperation are two other binary variables. 35.42\% of the sampled SMEs were engaged in cooperation with other firms, whereas a much smaller number of SME innovators (8.7\%) had such external relationships with universities or public research establishments. Furthermore, slightly more than half of the respondents (52.50\%) reported having generated revenue in 2006 from export sales, which is once again represented by a binary variable.

Another set of binary variables takes account of the relative firm size of the main competitors of SMEs (dummies: larger competitors, equal size competitors, smaller competitors and no main competitors). With regard to the corresponding hypothesis formulated above it is worth noting that $38.35 \%$ of innovative SMEs reported that they compete mainly with much larger 
firms. To assess the role of differences in industry we created four binary variables to cover the manufacturing, construction, trade and service sectors. Around 38\% of the sampled firms belonged to manufacturing industries, whereas almost $24 \%$ were assigned to the service sector. The total number of full-time employees in a responding company in 2006 is taken as an explanatory variable to verify the hypothesized firm size effects. In our sample of innovative SMEs, the average firm size amounts to 50.5 full-time employees. Finally, the age of a company was measured by the number of years since it had entered the market. The average firm in our sample is 34.96 years old.

Moreover, we added some control variables to the following regression analysis. A binary variable was created to control for the legal form of a company. In this regard, the results of Czarnitzki and Kraft (2004) have shown that owner-led SMEs tend to be less active innovators than manager-led SMEs, according to the authors probably because of a different incentive structure. A limited liability implying legal form may therefore be related to more intense innovation activities and hence a higher need for employing adequate means of innovation protection. In our sample, around 70\% of innovative SMEs are characterized by a corresponding legal form. Finally, to control for any structural bias, two binary variables indicate whether a respondent's company was located in East Germany and whether it had received any subsidies through a government support programme conducted by the KfW Bankengruppe during the reference period.

\section{Explanatory analysis and discussion}

Owing to the binary character of our dependent variables, a probit framework was an appropriate choice for the explanatory analysis. The same model specification was estimated separately for the formal and informal innovation protection mechanisms under consideration to determine the probability that an innovative SME perceived them in each case to be important. Probit regression results shown in Table 2 display the impact of the independent and control variables on the role of patents and other IPRs in innovation protection, while corresponding effects for informal protection methods are presented in Table 3. All models as a whole are statistically significant (Wald's Chi-squared Test for full model, significance level of $1 \%$ ), demonstrating the overall explanatory power of the chosen specification. Reported coefficients are marginal effects computed at the mean of the dataset. Finally, it has to be noted that the number of full-time employees and the firm age in years were taken in logarithms in order to better match them with the normal distribution.

To begin with, as expected, the existence of some form of in-house R\&D activity had a major influence on the perceived importance of the formal as well as almost all the informal protection mechanisms under consideration (see Table 2 and 3). Furthermore, in line with the corresponding hypothesis (H1.2), the relevance of patent protection strongly increased in relative terms when R\&D was conducted on a permanent basis. Yet, one result deserves special attention. The role of a staff-related appropriation strategy (i.e. the long-term retention of qualified personnel) seemed hardly affected by R\&D activities, and for this reason Hypothesis H1.1 is only partially confirmed. Hence, this protection mechanism also works effectively for innova- 
tive SMEs that refrain from own in-house R\&D (62.33\% of sampled firms). On the one hand, we interpret this result in the light of Rammer et al. (2009), who have shown that non-R\&Dperforming SMEs can gain a similar innovation success as R\&D performers when they focus on staff-related innovation management tools such as human resource management and team work, especially when these are combined with external knowledge sourcing or cooperation with external partners. On the other hand, we argue that this pattern probably reflects the necessity of using other innovation indicators beyond conventional measures such as R\&D-expenditures when one wants to capture more informal modes of innovation that tend to be based on tacit knowledge resulting from personal experience and learning by doing, using and interacting (see Tunzelmann/Acha 2005; Jensen et al. 2007; Santamaría et al. 2009). Support for this argument is found in the estimated effect of craft skills, as a typical type of tacit knowledge, on the dependent variables. Only in the probit regression performed on the importance of the long-term retention of qualified personnel, was the independent variable relating to craft-based know-how found to have a strong positive and significant impact. Thus, Hypothesis $\mathrm{H} 2$ is supported by the data.

Furthermore, it is confirmed that process-innovating SMEs are less likely to regard patent protection to be important (H3.1). Conversely, our results imply that secrecy protection, the complexity of design and the long-term retention of qualified personnel offer effective protection in the case of process innovation (H3.2). In this instance, it seems that the effects are slightly more pronounced in the case of the latter two informal mechanisms, which may point to the higher degree of complex and tacit knowledge incorporated in process innovations compared with the one in product innovations, as was argued in Section 2. This knowledgebased view of innovation type might also explain why process innovators' lower perception of the importance of formal protection methods not only applies to patents but also to other IPRs (see Table 2).

With regard to the role of cooperation activities, our regression results corroborate those of Leiponen and Byma (2009), since engagement in cooperation is shown to have a major impact on the kind of protection strategy chosen by innovative SMEs. Yet, in general it seems that this is less true for inter-firm cooperation, which is probably due to two factors (for the following see European Commission 2004): on the one hand, SMEs cooperate with other companies in many cases for other reasons than for access to core technological know-how in innovation, such as better market access, additional production capacity or the sharing of costs, a fact which should definitely increase the relevance of complementary assets as a preferred appropriation method for companies engaged in inter-firm cooperation (on the role of complementary sales, service and manufacturing capabilities in terms of appropriability, see Teece 1986 and Cohen et al. 2000). On the other hand, the cooperative inter-firm relations of SMEs frequently build on mutual trust. Hence, the overall need for employing the protection mechanisms under consideration in order to appropriate innovation returns could be lower in the case of inter-firm cooperation. 
Table 2 Importance of IPRs in innovation protection - results from binary probit analysis

\begin{tabular}{|c|c|c|}
\hline Independent variables & Patent & Other IPRs \\
\hline \multicolumn{3}{|c|}{ Reference: No in-house R\&D } \\
\hline Occasional in-house R\&D & $0.137(0.022)^{* * *}$ & $0.121(0.021)^{* * *}$ \\
\hline Permanent in-house R\&D & $0.248(0.023)^{* * *}$ & $0.134(0.022)^{* * *}$ \\
\hline Craft skills & $-0.009(0.015)$ & $0.002(0.015)$ \\
\hline Process innovator & $-0.042(0.014) * * *$ & $-0.043(0.014)^{* * *}$ \\
\hline Inter-firm cooperation & $-0.034(0.014)^{* *}$ & $0.020(0.014)$ \\
\hline Scientific cooperation & $0.053(0.026)^{* *}$ & $0.029(0.024)$ \\
\hline \multicolumn{3}{|c|}{ Reference: Larger competitors } \\
\hline Equal size competitors & $-0.045(0.014) * * *$ & $-0.039(0.014)^{* * *}$ \\
\hline Smaller competitors & $0.015(0.025)$ & $0.018(0.024)$ \\
\hline No main competitors & $-0.032(0.032)$ & $-0.044(0.030)$ \\
\hline Export activities & $0.049(0.015)^{* * *}$ & $0.071(0.015)^{* * *}$ \\
\hline \multicolumn{3}{|l|}{ Reference: Manufacturing } \\
\hline Construction & $-0.003(0.024)$ & $-0.048(0.022)^{* *}$ \\
\hline Trade & $-0.046(0.017) * * *$ & $0.015(0.018)$ \\
\hline Services & $-0.094(0.016)^{* * *}$ & $-0.078(0.016)^{* * *}$ \\
\hline Log (employees) & $0.013(0.006)^{* *}$ & $-0.007(0.006)$ \\
\hline Log (firm age) & $-0.030(0.007)^{* * *}$ & $0.009(0.007)$ \\
\hline \multicolumn{3}{|l|}{ Control variables } \\
\hline Limited liability & $0.023(0.017)$ & $0.023(0.017)$ \\
\hline East Germany & $0.000(0.014)$ & $-0.009(0.014)$ \\
\hline Subsidy status & $-0.003(0.014)$ & $-0.024(0.014)^{*}$ \\
\hline Number of observations & 4162 & 4162 \\
\hline Wald Chi-square (18) & $429.95^{* * *}$ & $244.33^{* * *}$ \\
\hline Log pseudo-likelihood & -2044.12 & -2050.69 \\
\hline
\end{tabular}

Note: Estimated coefficients are marginal effects from probit regressions with robust standard errors in parentheses. Marginal effects are computed at the variable means. In the case of binary variables the marginal effect measures the change in probability when the dummy changes from 0 to 1 .

$* * *$ report a significance level of $1 \%$, ** of $5 \%$ and * of $10 \%$ 
Table 3 Importance of informal mechanisms in innovation protection - results from binary probit analysis

\begin{tabular}{|c|c|c|c|c|}
\hline Independent variables & Secrecy & $\begin{array}{l}\text { Complexity of } \\
\text { design }\end{array}$ & $\begin{array}{l}\text { Long-term } \\
\text { retention of } \\
\text { qualified } \\
\text { personnel }\end{array}$ & $\begin{array}{l}\text { Lead time } \\
\text { advantage }\end{array}$ \\
\hline \multicolumn{5}{|l|}{$\begin{array}{l}\text { Reference: No in-house } \\
\text { R\&D }\end{array}$} \\
\hline Occasional in-house R\&D & $0.119(0.023)^{* * *}$ & $0.069(0.019)^{* * *}$ & $0.001(0.023)$ & $0.103(0.021)^{* * *}$ \\
\hline Permanent in-house R\&D & $0.135(0.022)^{* * *}$ & $0.116(0.020)^{* * *}$ & $0.042(0.023)^{*}$ & $0.144(0.022)^{* * *}$ \\
\hline Craft skills & $-0.017(0.017)$ & $0.011(0.014)$ & $0.071(0.018) * * *$ & $-0.009(0.019)$ \\
\hline Process innovator & $0.038(0.015)^{* *}$ & $0.040(0.012)^{* * *}$ & $0.049(0.016)^{* * *}$ & $0.016(0.016)$ \\
\hline Inter-firm cooperation & $-0.004(0.015)$ & $0.028(0.013)^{* *}$ & $0.015(0.017)$ & $0.055(0.017)^{* * *}$ \\
\hline Scientific cooperation & $0.081(0.028)^{* * *}$ & $0.002(0.021)$ & $0.136(0.030)^{* * *}$ & $0.122(0.029)^{* * *}$ \\
\hline \multicolumn{5}{|l|}{$\begin{array}{l}\text { Reference: Larger } \\
\text { competitors }\end{array}$} \\
\hline Equal size competitors & $-0.033(0.016)^{* *}$ & $-0.009(0.013)$ & $0.008(0.017)$ & $0.000(0.017)$ \\
\hline Smaller competitors & $-0.025(0.026)$ & $-0.001(0.022)$ & $0.027(0.029)$ & $0.053(0.028) *$ \\
\hline No main competitors & $-0.108(0.031)^{* * *}$ & $0.020(0.033)$ & $-0.012(0.042)$ & $-0.020(0.041)$ \\
\hline Export activities & $0.082(0.016)^{* * *}$ & $0.046(0.014)^{* * *}$ & $0.038(0.018)^{* *}$ & $0.029(0.018)$ \\
\hline \multicolumn{5}{|l|}{ Reference: Manufacturing } \\
\hline Construction & $-0.076(0.024)^{* * *}$ & $-0.002(0.021)$ & $0.007(0.029)$ & $0.010(0.029)$ \\
\hline Trade & $-0.068(0.019)^{* * *}$ & $-0.069(0.015)^{* * *}$ & $0.030(0.022)$ & $0.020(0.022)$ \\
\hline Services & $-0.092(0.019)^{* * *}$ & $-0.052(0.015)^{* * *}$ & $0.060(0.023)^{* * *}$ & $-0.042(0.023)^{*}$ \\
\hline Log (employees) & $0.010(0.007)$ & $-0.002(0.006)$ & $0.037(0.007)^{* * *}$ & $0.017(0.007)^{* *}$ \\
\hline Log (firm age) & $-0.027(0.008)^{* * *}$ & $-0.011(0.007)^{*}$ & $-0.030(0.008)^{* * *}$ & $-0.017(0.008)^{* *}$ \\
\hline \multicolumn{5}{|l|}{ Control variables } \\
\hline Limited liability & $-0.022(0.019)$ & $0.021(0.015)$ & $0.020(0.020)$ & $0.050(0.020)^{* *}$ \\
\hline East Germany & $-0.015(0.016)$ & $-0.019(0.013)$ & $-0.042(0.017)^{* *}$ & $-0.065(0.017)^{* * *}$ \\
\hline Subsidy status & $-0.019(0.015)$ & $0.021(0.012)^{*}$ & $0.017(0.016)$ & $0.013(0.016)$ \\
\hline Number of observations & 4162 & 4162 & 4162 & 4162 \\
\hline Wald Chi-square (18) & $319.96^{* * *}$ & $218.84^{* * *}$ & $145.69 * * *$ & $233.09 * * *$ \\
\hline Log pseudo-likelihood & -2344.66 & -1855.71 & -2756.88 & -2689.45 \\
\hline
\end{tabular}

Note: Estimated coefficients are marginal effects from probit regressions with robust standard errors in parentheses. Marginal effects are computed at the variable means. In the case of binary variables the marginal effect measures the change in probability when the dummy changes from 0 to 1 .

$* * *$ report a significance level of $1 \%, * *$ of $5 \%$ and $*$ of $10 \%$ 
In detail, the following results were obtained with regard to the role of cooperation activities: firstly, as expected (H4.1), both forms of external partnership had a positive and significant effect on the likelihood of following a lead time strategy, which reflects the important contribution of cooperation to the competitive strength of SMEs. Secondly, engagement in cooperation with universities or public research establishments strongly increased the relevance of the long-term retention of qualified personnel, a fact that was not observed in the case of interfirm cooperation activities. Thus, Hypothesis H4.2 is only partially confirmed. Thirdly, in accordance with Hypothesis H4.3, innovative SMEs deemed patent protection to be more important when they were engaged in scientific cooperation. Fourthly, some noteworthy results are not included in any hypothesis. Surprisingly, innovative companies engaged in inter-firm cooperation were less likely to regard patents as an important protection mechanism, a result which may perhaps reflect the frequently trust-based and non-formalized nature of inter-firm collaborations among SMEs. However, deeper analysis would be necessary to justify this interpretation. Instead, we found some evidence that the complexity of design works as an important protection mechanism for companies with such external relationships. The reason for this outcome could be traced back to the fact that knowledge complexity as a barrier to imitation arises from the degree of multiple competencies that are combined to provide a new or significantly improved product (Reed/DeFillippi 1990; Zander/Kogut 1995). Apparently for this reason, a protection strategy that builds on complexity of design becomes more effective for SME innovators when they cooperate with other companies. Finally, scientific cooperation is observed to have a positive and significant impact on the relevance of secrecy protection, which, once again, shows how critical this external source of technological innovation is to some parts of the SME sector.

With respect to the relative size of a company's main competitors, it was argued that innovative SMEs which compete with large firms attribute higher importance to IPR protection. In fact, this is what we observe, since SMEs competing mainly with similar-sized companies are less likely to rely on patents or other IPRs for innovation protection than those facing much larger competitors (significant at 0.01). Thus, Hypothesis H5.1 is confirmed. In this instance, the results for innovative SMEs which mainly compete with smaller competitors (true for 9\% of companies in our sample) are also worth noting. The positive sign of this relation in comparison to the reference case is in line with our argument that larger firms can be at an advantage compared with their smaller-sized competitors when it comes to the use of IPRs. Yet, the magnitude of this effect is small, with no statistical significance, confirming that the nature of competitive interaction as suggested by Hypothesis H5.1 is really one between SMEs and large firms and less between SMEs of different size classes. Hypothesis H5.2, however, is only weakly supported by the data. Only in the case of secrecy did we find some evidence that SMEs tend to favour this type of informal protection mechanism more strongly when they have to compete with larger firms.

The regression results further imply that export activities are related to a higher relevance of both formal and informal protection mechanisms. Only in the case of lead time is no significant effect found, although the sign is positive as expected. Thus, there is evidence in sup- 
port of Hypothesis H6. But the interpretation of this effect remains ambiguous since the importance of an adequate appropriation strategy as perceived by exporting SMEs may either be caused naturally by their general innovativeness or result from the higher risk of imitation in highly competitive export markets (see Section 2).

Concerning the supposed differences in innovation between service and manufacturing industries, the regression results confirm that innovative SMEs operating in the service sector are, on average, less likely to rely on IPR protection. Instead, they are more likely than manufacturing SMEs to perceive the long-term retention of qualified personnel as important for innovation protection (significant at 0.01). However, contrary to our expectation, this does not imply that informal protection practices are in general more relevant in services than in manufacturing. Apart from IPRs, the service innovators in our sample are also less likely to rely on secrecy, the complexity of design or lead time advantage (although with relatively low significance in the case of lead time). We interpret this result as an indication that, compared to manufacturing, the overall need for employing different formal and informal innovation protection mechanisms is lower in the service sector, probably because of the very intangible nature of many service products. Nevertheless, the strong human element in the successful provision of service innovation is reflected in a higher importance of a staff-related appropriation strategy for service SMEs. Hence, Hypothesis H7.1 is supported by the data, whereas Hypothesis H7.2 is only partially confirmed.

The effects of firm size on the importance of patent protection and lead time are positive and significant at 0.05 for both probit equations, pointing to certain economies of scale which are obviously associated with these types of innovation protection mechanism. In contrast, other IPRs, secrecy and the complexity of design seem not to be related to any size advantages, indicating that they can be used effectively by smaller as well as larger SMEs. Hence, there is empirical support for Hypothesis H8.2, whereas Hypothesis H8.1 is only confirmed in the case of patents. Furthermore, with respect to the likelihood of realising the long-term retention of qualified personnel, a positive and highly significant effect of firm size is found. No hypothesis was formulated in this regard since the use of this appropriation method should logically increase in absolute terms with the number of a company's employees. Yet, it should also be kept in mind that larger SMEs may be in a better position to maintain staff loyalty in an effective way than smaller ones, since their larger resource base perhaps enables them to provide stronger incentives to their employees (e.g. those related to wages, training opportunities or potential career paths).

The age of an innovative SME is observed to have a negative and significant impact on the perceived importance of all formal and informal protection mechanisms under consideration in this study, except that related to other IPRs. Hence, we found evidence in support of Hypothesis H9 that the higher innovation propensity of younger SMEs is linked to a particular emphasis on effective appropriation strategies for profiting sufficiently from innovation returns. 


\section{Conclusion and implications for policy and research}

This paper has examined the determinants of innovation protection practices in small and medium enterprises (SMEs). Discounting formal mechanisms, that is, intellectual property rights (IPRs), it places a particular emphasis on informal methods, so as to take into account the diversity of smaller firms when it comes to appropriation strategies. On the one hand, our study contributes to the existing literature by using a large data set of innovative SMEs. This way we are able to verify several hypotheses concerning the importance perceived by SMEs of different innovation protection mechanisms on a broad empirical base. On the other hand, by starting from a knowledge-based view, the paper aims to provide a better understanding of certain SME protection practices. The latter is especially true for the long-term retention of qualified personnel, a staff-related appropriation strategy whose explanatory determinants have not been addressed in previous studies. Among other informal methods such as secrecy or lead time, this mechanism should be of particular concern for many smaller firms, owing to their more informal ways of learning and innovation resulting in experience-based know-how with strong tacit elements. In fact, support for this argument is already provided by Kitching and Blackburn (1998; 2003) who have shown that by far the largest proportion of SME owners, in their telephone sample, perceived the departure of key staff as the most significant threat to their specialist and confidential knowledge (e.g. compared to threats from competitors, collaborators, customers or suppliers). In a similar manner, in conducting case studies on eight Finnish SMEs, Olander et al. (2009) came up with the proposition that human resource management is an essential appropriation method for innovative SMEs, since the personembodied nature of their knowledge base makes the loss of key employees a particular risk.

Our descriptive results underline the importance of informal protection practices for innovative SMEs. Lead time was the most commonly used mechanism (59.39\%), followed by the long-term retention of qualified personnel (41.98\%), secrecy (29.05\%) and the complexity of design (18.02\%). However, the descriptive statistics further indicate that patents (23.57\%) and other IPRs (21.77\%) can also be an important means of innovation protection for subgroups of SMEs.

The explanatory analysis has shown that several characteristics of innovative SMEs help to explain their preference for different protection mechanisms. The main findings can be summarised as follows. Firstly, we have observed a positive impact of in-house R\&D activities on both formal and informal innovation protection practices, pointing to the high relevance of $R \& D$ for generating new knowledge. Yet, the expected diversity among smaller firms with regard to R\&D (for more detail see Ortega-Argilés et al. 2009) is shown by the fact that the long-term retention of qualified personnel can also work effectively for non-R\&D performers (i.e. the majority of our sample). We interpret this as an indication of the relatively little formal learning by science and technology in large parts of the SME sector. Secondly, in line with the aforementioned argument, a staff-related appropriation strategy has been observed to provide effective protection under certain circumstances. Notably, this is true in the case of craft-based SMEs and those from service industries owing to the prominent role of 
tacit knowledge in their innovation context. Furthermore, for similar reasons, the long-term retention of qualified personnel seems to be more important for process innovators (together with secrecy and the complexity of design) and for innovative SMEs engaged in scientific cooperation with universities or public research establishments (together with secrecy and lead time). Maintaining a lead time advantage over competitors is also more achievable for larger SMEs and those engaged in inter-firm cooperation. Thirdly, we have found some evidence that the codified results of scientific cooperation activities are protected via patents. Moreover, larger SMEs and, above all, those that conduct R\&D on a permanent basis rely more heavily on patent protection. An interesting finding regarding the overall role of IPR protection for innovative SMEs is that it matters whether they compete with large firms or not. The corresponding results indicate that smaller firms must place a stronger emphasis on patents and other IPRs in order to appropriate their innovation returns successfully when their main competitors are much larger in size. Fourthly, younger as well as exporting SMEs tend to attribute a higher importance to innovation protection practices in general, which probably points to the fact that these firms are particularly challenged by potential imitation.

Our results have some implications for innovation policy. By examining the explanatory determinants of several formal and informal protection mechanisms, the present study has once again shown the heterogeneous nature of smaller firms. Hence, in order to fully recognize this diversity, a targeted policy approach is needed to improve the competitiveness of SMEs in terms of appropriability. On the one hand, there is thus a case for policy initiatives that aim to foster the use by SMEs of formal protection mechanisms by an easier and cheaper access to IPRs and a better enforceability of these legal rights in the case of infringement. Yet, policymakers should remind themselves that particular subgroups of innovative SMEs such as R\&D-performers or start-up firms may benefit more from this public support than others. Hence, innovation policy may, for example, focus on SMEs engaged in competition with large firms since there is empirical evidence that these have great difficulties enforcing their patents successfully in the case of infringement (see Kingston 2000). On the other hand, policy should also take informal means of appropriation into account. For example, initiatives in support of SME cooperation seem to be justified by the fact that a lead time advantage over competitors is more achievable for innovative SMEs that collaborate with other firms or scientific institutions, reflecting the crucial contribution of cooperation activities to the competitive position of SMEs. Moreover, policy-makers may try to remove potential barriers that prevent smaller firms from effectively adopting a staff-related appropriation strategy (e.g. by providing information on the use of human resource management tools). Finally, in more general terms our results imply that, in order not to neglect the strong emphasis placed by smaller firms on informal processes of learning and experience-based know-how, government policies addressing the specific needs of innovative SMEs should focus not only on conventional measures such as formal R\&D-expenditure or the number of patent applications.

One limitation of our study is that, because of the non-availability of panel data on the topic under consideration, it is restricted to cross-section analysis. Furthermore, we did not focus on the complementarities between certain innovation protection mechanisms. An examination of 
their possible interplay seems promising, since the strength of single appropriation methods often lies in their combined use (Bosworth/Webster 2006). On the basis of our results, another path for future research would be to seek a deeper understanding of the appropriation behaviour of SMEs with regard to their human resource management strategies, for example by choosing a qualitative approach. Moreover, the double-sided nature of a company's tacit knowledge base might be addressed by future research. The present study has highlighted the beneficial effects of tacitness when it comes to the protection of innovation results from unwanted imitation. However, limited knowledge transferability is not always preferable for a company in economic terms. This may, for example, be true in cases where the replication and sharing of knowledge within firms or between partners generates collective and cumulative learning as a basis for further innovation (Hurmelinna et al. 2007). Thus, to the extent that knowledge in smaller firms tends to be more tacit than in larger firms, the role of tacitness for innovative SMEs is worth more detailed study. 


\section{Appendix A}

Table A1 Descriptive statistics of independent and control variables (reference period: 20042006, $N=4162$ )

\begin{tabular}{|c|c|c|c|}
\hline Continuous Variables & Description & Mean & S.D. \\
\hline Employees & Number of full-time employees in 2006 & 50.49 & 70.73 \\
\hline Firm age & Years since market entry & 34.96 & 40.03 \\
\hline Binary Variables & Description & \multicolumn{2}{|r|}{ Percentage } \\
\hline No in-house R\&D & 1 if firm conducted no in-house R\&D, 0 otherwise & \multicolumn{2}{|r|}{62.33} \\
\hline Occasional in-house R\&D & $\begin{array}{l}1 \text { if firm conducted in-house } \mathrm{R} \& \mathrm{D} \text { on an occasional } \\
\text { basis, } 0 \text { otherwise }\end{array}$ & \multicolumn{2}{|r|}{16.63} \\
\hline Permanent in-house R\&D & $\begin{array}{l}1 \text { if firm conducted in-house } R \& D \text { on a permanent ba- } \\
\text { sis, } 0 \text { otherwise }\end{array}$ & \multicolumn{2}{|r|}{21.05} \\
\hline Craft skills & $\begin{array}{l}1 \text { if firm considers its main activities to be craft-based, } \\
0 \text { otherwise }\end{array}$ & \multicolumn{2}{|r|}{35.92} \\
\hline Process innovator & 1 if firm introduced process innovations, 0 otherwise & \multicolumn{2}{|r|}{61.97} \\
\hline Inter-firm cooperation & $\begin{array}{l}1 \text { if firm is engaged in cooperation with other firms, } 0 \\
\text { otherwise }\end{array}$ & \multicolumn{2}{|r|}{35.42} \\
\hline Scientific cooperation & $\begin{array}{l}1 \text { if firm is engaged in cooperation with universities or } \\
\text { public research establishments, } 0 \text { otherwise }\end{array}$ & \multicolumn{2}{|r|}{8.70} \\
\hline Larger competitors & $\begin{array}{l}1 \text { if firm's main competitors are much larger, } 0 \text { other- } \\
\text { wise }\end{array}$ & \multicolumn{2}{|r|}{38.35} \\
\hline Equal size competitors & $\begin{array}{l}1 \text { if firm's main competitors are of more or less equal } \\
\text { size, } 0 \text { otherwise }\end{array}$ & \multicolumn{2}{|r|}{48.85} \\
\hline Smaller competitors & $\begin{array}{l}1 \text { if firm's main competitors are much smaller, } 0 \\
\text { otherwise }\end{array}$ & \multicolumn{2}{|r|}{8.94} \\
\hline No main competitors & $\begin{array}{l}1 \text { if firm reported having no main competitors, } 0 \\
\text { otherwise }\end{array}$ & \multicolumn{2}{|r|}{3.87} \\
\hline Export activities & 1 if firm had any exports in 2006, 0 otherwise & \multicolumn{2}{|r|}{52.50} \\
\hline Manufacturing & $\begin{array}{l}1 \text { if firm belongs to the manufacturing sector, } 0 \text { other- } \\
\text { wise }\end{array}$ & \multicolumn{2}{|r|}{38.23} \\
\hline Construction & $\begin{array}{l}1 \text { if firm belongs to the construction sector, } 0 \text { other- } \\
\text { wise }\end{array}$ & \multicolumn{2}{|r|}{11.48} \\
\hline Trade & 1 if firm belongs to the trade sector, 0 otherwise & \multicolumn{2}{|r|}{26.38} \\
\hline Services & 1 if firm belongs to the service sector, 0 otherwise & \multicolumn{2}{|r|}{23.91} \\
\hline Limited liability & $\begin{array}{l}1 \text { if firm's legal form implies limited liability of the } \\
\text { owners, } 0 \text { otherwise }\end{array}$ & \multicolumn{2}{|r|}{70.21} \\
\hline East Germany & 1 if firm is located in East Germany, 0 otherwise & \multicolumn{2}{|r|}{35.42} \\
\hline Subsidy status & 1 if firm received subsidies, 0 otherwise & \multicolumn{2}{|r|}{64.56} \\
\hline
\end{tabular}




\section{Literature}

Amara, N., R. Landry, N. Traoré (2008), Managing the protection of innovations in knowledge-intensive business services. Research Policy 37 (9): 1530-1547.

Arrow, K. J. (1962), Economic welfare and the allocation of resources for invention. Pp. 609626 in: R. R. Nelson (ed.), The rate and direction of inventive activity: economic and social factors, Princeton, NJ.

Arundel, A. (2000), Patents - the viagra of innovation policy?, internal report for the project "Innovation Policy in a Knowledge-Based Economy" commissioned by the European Commission. Retrieved from http://www.edis.sk/ekes/patents.pdf (Accessed May 19, 2011).

Arundel, A. (2001), The relative effectiveness of patents and secrecy for appropriation. Research Policy 30 (4): 611-624.

Arundel, A., I. Kabla (1998), What percentage of innovations are patented? Empirical estimates for European firms. Research Policy 27: 127-141.

Baldwin, J., G. Gellatly (2003), Innovation strategies and performance in small firms. Cheltenham, UK.

Baldwin, J., J. Johnson (1996), Business strategies in more- and less-innovative firms in Canada. Research policy 25 (5): 785-804.

Bekkers, R. (2008), Analysing knowledge transfer channels between universities and industry: To what degree do sectors also matter? Research policy 37 (10): 1837-1853.

Beyer, M., M. Stephan (2008), Schutzstrategien für produktbegleitende Dienstleistungsinnovationen Pp. 195-222 in: A. Eisenkopf, C. Opitz, H. Proff (eds.), Strategisches Kompetenz-Management in der Betriebswirtschaftslehre. Eine Standortbestimmung, Wiesbaden.

Blind, K., J. Edler, R. Frietsch, U. Schmoch (2006), Motives to patent: Empirical evidence from Germany. Research Policy 35 (5): 655-672.

Blind, K., J. Edler, U. Schmoch, B. Anderson, J. Howells, I. Miles, J. Roberst, L. Green, R. Evangelista, C. Hipp, C. Herstatt (2003), Patents in the service industries. Final report for the European Commission. Fraunhofer-ISI, Karlsruhe, Germany. Retrieved from ftp://ftp.cordis.europa.eu/pub/indicators/docs/ind_report_fraunhofer1.pdf (Assessed April 1, 2011).

Bosworth, D., E. Webster (2006), An economic perspective. Pp. 85-107 in: D. Bosworth, E. Webster (eds.), The management of intellectual property, Cheltenham, UK.

Bougrain a, F., B. Haudeville (2002), Innovation, collaboration and SMEs internal research capacities. Research Policy 31: 735-747.

Brouwer, E., A. Kleinknecht (1999), Innovative output, and a firm's propensity to patent. - An exploration of CIS micro data. Research Policy 28 (6): 615-624.

Brusoni, S., O. Marsili, A. Salter (2005), The role of codified sources of knowledge in innovation: empirical evidence from Dutch manufacturing. Journal of Evolutionary Economics 15: 211-231.

Burr, W., M. Stephan, B. Soppe, S. Weisheit (2007), Patentmanagement - Strategischer Einsatz und ökonomische Bewertung von technologischen Schutzrechten. Stuttgart. 
Cantwell, J. (2005), Innovation and competitiveness. Pp. 543-567 in: J. Fagerberg, D. C. Mowery, R. R. Nelson (eds.), The Oxford Handbook of Innovation, Oxford.

Cohen, W. M., D. A. Levinthal (1989), Innovation and learning: the two faces of R\&D. The Economic Journal 99: 569-596.

Cohen, W. M., D. A. Levinthal (1990), Absorptive capacity: a new perspective on learning and innovation. Administrative Science Quarterly 35: 128-152.

Cohen, W. M., R. R. Nelson, J. P. Walsh (2000), Protecting their intellectual assets: appropriability conditions and why U.S. manufacturing firms patent (or Not). NBER Working Papers Series (7552).

Czarnitzki, D., K. Kraft (2004), Firm leadership and innovative performance: evidence from seven EU countries. Small Business Economics 22 (5): 325-332.

De Faria, P., W. Sofka (2010), Knowledge protection strategies of multinational firms. A cross-country comparison. Research Policy 39 (7): 956-968.

European Commission (2004), SMEs and cooperation. Observatory of European SMEs 2003, No. 5. Retrieved from http://ec.europa.eu/enterprise/policies/sme/files/analysis/doc/smes_observatory_2003_rep ort5_en.pdf (Assessed April 17, 2011).

Galende, J. (2006), The appropriation of the results of innovative activity. International Journal of Technology Management 35: 107-135.

Gamble, J. (2001), Modelling the Invisible: the pedagogy of craft apprenticeship. Studies in Continuing Education 23 (2): 185-200.

Gamble, J. (2004), Retrieving the general from the particular. The structure of craft knowledge. Pp. 189-203 in: J. Muller, B. Davies, A. Morais (eds.), Reading Bernstein, Researching Bernstein, London, New York.

Gans, J. S., D. H. Hsu, S. Stern (2002), When Does Start-Up Innovation Spur the Gale of Creative Destruction? RAND Journal of Economics 33 (4): 571-586.

Gans, J. S., S. Stern (2003), The product market and the market for "ideas": commercialization strategies for technology entrepreneurs. Research Policy 32 (2): 333350.

Geroski, P. (1995), Markets for Technology: Knowledge, Innovation and Appropriability. Pp. 90-131 in: P. Stoneman (ed.), Handbook of the Economics of Innovation and Technological Change, Oxford.

González-Álvarez, N., M. Nieto-Antolín (2007), Appropriability of innovation results: an empirical study in spanish manufacturing firms. Technovation 27 (5): 280-295.

Gopalakrishnan, S., P. Bierly, E. H. Kessler (1999), A reexamination of product and process innovations using a knowledge-based view. The journal of high technology management research 10 (1): 147-166.

Greenhalgh, C. A., M. Rogers (2007), Intellectual property activity by service sector and manufacturing firms in the United Kingdom, 1996 - 2000. Pp. 295-317 in: H. Scarbrough (ed.), The evolution of business knowledge, Oxford.

Hadjimanolis, A. (2000), A resource-based view of innovativeness in small firms. Technology Analysis \& Strategic Management 12 (2): 263-281.

Hall, B. H., F. Lotti, J. Mairesse (2009), Innovation and productivity in SMEs: empirical evidence for Italy. Small Business Economics 33: 13-33. 
Hall, M., C. Oppenheim, M. Sheen (2003), Barriers to the use of patent information in SMEs. Pp. 144-160 in: R. A. Blackburn (ed.), Intellectual property and innovation management in small firms, London/New York.

Hanel, P. (2008), The use of intellectual property rights and innovation by manufacturing firms in Canada. Economics of Innovation and New Technology 17 (3): 285-309.

Harabi, N. (1995), Appropriability of technical innovations. An empirical analysis. Research Policy 24 (6): 981-992.

Hewitt-Dundas, N. (2006), Resource and capability constraints to innovation in small and large plants. Small Business Economics 26: 257-277.

Howells, J. (1996), Tacit knowledge, innovation and technology transfer. Technology Analysis \& Strategic Management 8 (2): 91-106.

Huergo, E., J. Jaumandreu (2004), How does probability of innovation change with firm age? Small Business Economics 22 (3/4): 193-207.

Hurmelinna-Laukkanen, P. (2009), The availability, strength and efficiency of appropriability mechanisms - protecting investments in knowledge creation. International Journal of Technology Management 45 (3): 282-290.

Hurmelinna-Laukkanen, P., K. Puumalainen (2007), Nature and dynamics of appropriability: strategies for appropriating returns on innovation. R\&D Management 37 (2): 95-112.

Hurmelinna, P., K. Kyläheiko, T. Jauhiainen (2007), The Janus face of the appropriability regime in the protection of innovations: Theoretical re-appraisal and empirical analysis. Technovation 27 (3): 133-144.

Jensen, M. B., B. Johnson, E. Lorenz, B. Å. Lundvall (2007), Forms of knowledge and modes of innovation. Research Policy 36 (5): 680-693.

Kingston, W. (2000). Enforcing small firms' patent rights. Research report funded by the European Commission. Trinity College, Dublin, Ireland. Retrieved from ftp://ftp.cordis.europa.eu/pub/innovationpolicy/studies/studies_enforcing_firms_patent_rights.pdf (Assessed March 21, 2011).

Kitching, J., R. A. Blackburn (1998), Intellectual property management in the small and medium enterprise (SME). Journal of Small Business and Enterprise Development 5 (4): 327-335.

Kitching, J., R. A. Blackburn (2003), Innovation, intellectual property and informality: evidence from a study of small enterprises and some implications for policy. Pp. 16-34 in: R. A. Blackburn (ed.), Intellectual property and innovation management in small firms, London/New York.

Kleinknecht, A. H. (1987), Measuring R\&D in small firms: how much are we missing? The Journal of Industrial Economics 36 (2): 253-256.

Kleinknecht, A. H. (1991), More evidence on the undercounting of small firm R\&D. Research Policy 20 (6): 579-587.

Lanjouw, J. O., M. Schankerman (2004), Protecting intellectual property rights: Are small firms handicapped? The Journal of Law \& Economics 47 (1): 45-74.

Leiponen, A. (2005), Organization of knowledge and innovation: The case of Finnish business services. Industry and Innovation 12 (2): 185-203.

Leiponen, A., J. Byma (2009), If you cannot block, you better run: Small firms, cooperative innovation, and appropriation strategies. Research Policy 38 (9): 1478-1488. 
Levin, R. C., A. K. Klevorick, R. R. Nelson, S. G. Winter (1987), Appropriating the returns from industrial research and development. Brookings Papers on Economic Activity 3: 783-831.

Levy, R., P. Roux, S. Wolff (2009), An analysis of science-industry collaborative patterns in a large European University. The Journal of Technology Transfer 34 (1): 1-23.

López, A. (2009), Innovation and appropriability: Empirical evidence and research agenda. Pp. 1-32 in: World Intellectual Property Organization (ed.), The economics of intellectual property. Suggestions for further research in developing countries and countries with economies in transition. Retrieved from http://www.wipo.int/export/sites/www/ipdevelopment/en/economics/pdf/wo_1012_e_ch_1.pdf (Accessed May 2, 2011).

Macdonald, S., B. Lefang (2003), Worlds apart: patent information and innovation in SMEs. Pp. 123-143 in: R. A. Blackburn (ed.), Intellectual property and innovation management in small firms, London/New York.

Matthews, D., J. Pickering, J. Kirkland (2003), A strategic approach to managing intellectual property. Pp. 35-54 in: R. A. Blackburn (ed.), Intellectual property and innovation management in small firms, London/New York.

Miles, I. (2005), Innovation in services. Pp. 433-458 in: J. Fagerberg, D. C. Mowery, R. R. Nelson (eds.), The Oxford Handbook of Innovation, Oxford.

Müller, E., V. Zimmermann (2009), The importance of equity finance for R\&D activity. Small Business Economics 33 (3): 303-318.

Nieto, M., C. Pérez-Cano (2004), The influence of knowledge attributes on innovation protection mechanisms. Knowledge and Process Management 11 (2): 117-126.

Nooteboom, B. (1994), Innovation and diffusion in small firms: Theory and evidence. Small Business Economics 6 (5): 327-347.

Norman, P. M. (2002), Protecting knowledge in strategic alliances. Resource and relational characteristics. The Journal of High Technology Management Research 13: 177-202.

OECD, Eurostat (2005), Oslo Manual. The measurement of scientific and technological activities. Guidelines for collecting and interpreting innovation data. Third edition. Paris.

Olander, H., P. Hurmelinna-Laukkanen, J. Mähönen (2009), What's small size got to do with it? Protection of intellectual assets in SMEs. International Journal of Innovation Management 13 (3): 349-370.

Ortega-Argilés, R., M. Vivarelli, P. Voigt (2009), R\&D in SMEs: a paradox? Small Business Economics 33: 3-11.

Päällysaho, S., J. Kuusisto (2008), Intellectual property protection as a key driver of service innovation: an analysis of innovative KIBS businesses in Finland and the UK. International Journal of Services Technology and Management 9 (3/4): 268-284.

Polanyi, M. (1958/1974), Personal knowledge. Towards a post-critical philosophy. Chicago.

Radauer, A., J. Streicher, F. Ohler (2007), Benchmarking National and Regional Support Services for SMEs in the Field of Intellectual and Industrial Property, Final Benchmarking Report on Behalf of the European Commission, Austrian Institute for SME Research. Retrieved from http://www.insme.org/documents/Benchmarking-ReportSME.pdf (Assessed March 21, 2011). 
Rammer, C., D. Czarnitzki, A. Spielkamp (2009), Innovation success of non-R\&Dperformers: substituting technology by management in SMEs. Small Business Economics 33 (1): 35-58.

Rantakyrö, L. (2005), Knowledge management in small firms. Global Business and Economics Review 7 (2/3): 214-225.

Reed, R., R. J. DeFillippi (1990), Causal ambiguity, barriers to imitation, and sustainable competitive advantage. Academy of Management Review 15 (1): 88-102.

Reize, F. (2007), KfW-Mittelstandspanel 2007. Mittelstand im Konjunkturhoch - Defizite bei Innovationen. Frankfurt am Main.

Rothwell, R. (1983), Innovation and firm size: a case for dynamic complementarity; or, is small really so beautiful? Journal of General Management 8 (3): 5-25.

Santamaría, L., M. J. Nieto, A. Barge-Gil (2009), Beyond formal R\&D: Taking advantage of other sources of innovation in low- and medium-technology industries. Research Policy 38 (3): 507-518.

Sattler, H. (2003), Appropriability of product innovations: An empirical analysis for Germany. International Journal of Technology Management 26 (5-6): 502-516.

Schartinger, D., C. Rammer, M. M. Fischer, J. Fröhlich (2002), Knowledge interactions between universities and industry in Austria: sectoral patterns and determinants. Research Policy 31 (3): 303-328.

Sennett, R. (2008), The craftsman. New Haven and London.

Teece, D. J. (1986), Profiting from technological innovation: Implications for integration, collaboration, licensing and public policy. Research Policy 15 (6): 285-305.

Teece, D. J. (2003), The strategic management of technology and intellectual property. Pp. 132-166 in: D. O. Faulkner, A. Campbell (eds.), The Oxford Handbook of Strategy; Volume I: A Strategy Overview and Competitive Strategy, Oxford.

Teece, D. J. (2006), Reflections on “Profiting from Innovation”. Research Policy 35 (8): 1131-1146.

Tether, B. S. (2005), Do services innovate (differently)? Insights from the European Innobarometer Survey. Industry and Innovation 12 (2): 153-184.

Tunzelmann von, N., V. Acha (2005), Innovation in "low-tech" industries. Pp. 407-432 in: J. Fagerberg, D. C. Mowery, R. R. Nelson (eds.), The Oxford Handbook of Innovation, Oxford.

Zander, U., B. Kogut (1995), Knowledge and the speed of the transfer and imitation of organizational capabilities: An empirical test. Organization Science 6 (1): 76-92.

Zimmermann, V. (2009), The Impact of Innovation on Employment in Small and Medium Enterprises with Different Growth Rates. Jahrbücher für Nationalökonomie und Statistik 229 (2-3): 313-326. 


\section{Kapitel V}

\section{Das Handwerk - ein Konjunkturstabilisator?}




\section{Inhalt}

1. Problemstellung: Stabilisator oder Krisenverstärker? 117

2. Überblick zum Forschungsstand 118

3. Die Krise 2008/2009: das Handwerk als Stabilitätsanker? 122

3.1 Lage der Gesamtwirtschaft und wirtschaftspolitische Reaktionen 122

3.2 Betroffenheit des Handwerks 126

4. Datenbasis 132

5. Allgemeine Konjunkturmuster im Handwerk 133

5.1 Methodisches Vorgehen 133

5.2 Stilisierte Fakten zur Handwerkskonjunktur 135

$\begin{array}{lll}5.3 & \text { Zwischenfazit } & 139\end{array}$

6. Maßgebliche Determinanten der Handwerkskonjunktur 142

6.1 Bestimmungsfaktoren der Nachfrage nach Handwerksleistungen 142

6.2 Konjunkturelle Trendentwicklungen im Vorfeld der Krise 148

6.3 Ausblick: Eine zukünftige Stabilisatorfunktion des Handwerks? 153

7. Zusammenfassung und Implikationen 155

$\begin{array}{ll}\text { Literaturverzeichnis } & 158\end{array}$ 


\section{Problemstellung: Stabilisator oder Krisenverstärker?}

Auch das Handwerk konnte sich der schweren Wirtschaftskrise 2008/2009 nicht entziehen. Nach den vorliegenden Berechnungen gingen im Jahr 2009 die nominalen Umsätze im zulassungspflichtigen Handwerk gegenüber dem Vorjahr um 5,0 \% zurück. ${ }^{1}$ Prozentual gesehen erlebte die Handwerkswirtschaft damit einen stärkeren Rückgang als das nominale Bruttoinlandsprodukt (-3,5 \% gegenüber 2008). ${ }^{2}$ Auf der anderen Seite wurde jedoch in ersten Einschätzungen hervorgehoben, dass das Handwerk und darüber hinaus der gewerbliche Mittelstand insgesamt zwar stark von den Auswirkungen der Krise betroffen waren. Alles in allem hätten sie aber in dieser kritischen Phase - anders als die Entwicklung des Handwerksumsatzes im Krisenjahr 2009 vermuten lässt - eine aus volkswirtschaftlicher Sicht wichtige konjunkturelle Stabilisatorfunktion innegehabt. ${ }^{3}$ Vor diesem Hintergrund geht der vorliegende Beitrag der Frage nach, inwieweit die Handwerkswirtschaft tatsächlich - mit Blick auf den aktuellen Fall und allgemein - eine konjunkturelle Stabilisatorfunktion besitzt und welche Folgerungen sich hieraus für die Wirtschaftspolitik ergeben.

Nachdem Kapitel 2 zunächst einen Überblick zum Forschungsstand liefert, wird in Kapitel 3 der Krisenverlauf in der deutschen Realwirtschaft sowohl aus gesamtwirtschaftlicher Perspektive als auch aus Sicht des Handwerks nachgezeichnet. In diesem Kapitel soll vor allem die Frage geklärt werden, ob das Handwerk in der jüngsten Wirtschaftskrise als Konjunkturstabilisator gewirkt hat oder nicht. Nachdem in Kapitel 4 die Datengrundlage der anschließenden Analyse dargestellt wurde, zeigt Kapitel 5 mit Hilfe einer zeitreihenanalytischen Auswertung von Beschäftigungs- und Umsatzdaten der Handwerksberichterstattung (Zeitraum 1996 bis 2009) stilisierte Fakten zur Handwerkskonjunktur auf. Hierbei geht es um statistisch messbare Zusammenhänge zwischen dem gesamtwirtschaftlichen Konjunkturzyklus und der wirtschaftlichen Entwicklung in einzelnen Bereichen der Handwerkswirtschaft. Dieser Vergleich erlaubt bereits erste Rückschlüsse bezüglich der Tatsache, dass die einzelnen Bereiche des Handwerks im Krisenjahr 2009 unterschiedlich stark von der Rezession betroffen waren. Auf dieser Grundlage verdeutlicht wiederum Kapitel 6 die zentralen gesamtwirtschaftlichen Determinanten der Nachfrage nach Handwerksleistungen. An dieser Stelle ist demnach zu klären, wovon die konjunkturellen Bewegungen im Handwerk unter volkswirtschaftlichen Gesichtspunkten maßgeblich abhängen. Vor dem Hintergrund dieser Erkenntnisse wird der konjunkturelle Trendverlauf der Handwerkswirtschaft im Vorfeld der Krise 2008/2009 eingeschätzt. Hierdurch soll zum einen deutlich werden, wie sich der spezifische Charakter einer konjunkturellen Gesamtkonstellation auf die jeweilige Wirtschaftslage im Handwerk

1 Vgl. Statistisches Bundesamt, Pressemitteilung Nr. 110 vom 19.03.2010, „5,0\% weniger Umsatz im Handwerk im Jahr 2009“, in: http://www.destatis.de (letzter Zugriff: 25.05.2010). Parallel dazu waren im zulassungspflichtigen Handwerk 1,5 \% weniger Personen beschäftigt als im Vorjahr.

2 Da die Handwerksumsätze nur in nominaler (d.h. nicht-preisbereinigter) Form vorliegen, wird das nominale Bruttoinlandsprodukt als Vergleichsmaßstab herangezogen.

3 Vgl. IW-Köln (2010) und KfW u. a. (2010), S. 5ff. 
auswirkt. Zum anderen soll auf diesem Wege berücksichtigt werden, dass die vergangene wirtschaftliche Entwicklung im Handwerk neben eher kurzfristigen konjunkturellen Bewegungen vor allem auch durch längerfristige strukturelle Entwicklungsprozesse geprägt war. Nachdem des Weiteren der Frage nachgegangen wurde, inwieweit zukünftig eher eine konjunkturelle Stabilisatorfunktion des Handwerks zu erwarten ist, schließt Kapitel 7 mit einer Zusammenfassung der Ergebnisse und einer abschließenden Beantwortung der Ausgangsfrage. Zudem werden die verschiedenen Implikationen der gewonnenen Untersuchungsergebnisse formuliert. ${ }^{4}$

\section{2. Überblick zum Forschungsstand}

Es liegen nur wenige Untersuchungen vor, die explizit der Frage nach der konjunkturstabilisierenden Funktion des Handwerks nachgegangen sind. ${ }^{5}$ Sie nähern sich der Thematik dabei durch eine Messung der statistischen Zusammenhänge zwischen dem gesamtwirtschaftlichen Konjunkturverlauf und der wirtschaftlichen Entwicklung des Handwerks.

Vor dem Hintergrund der Krisenjahre 1966/1967 und 1974/1975 legte Schmidt (1975) die bisher umfangreichste Untersuchung vor. Schmidt setzte sich hierbei zum Ziel, auf der Grundlage von konjunkturstatistischen Daten zwei, bereits zum damaligen Zeitpunkt diskutierte Hypothesen einer Überprüfung zu unterziehen. Gemäß der sog. „Stabilisatorhypothese“ trägt das kleinbetrieblich geprägte Handwerk aufgrund seiner höheren Flexibilität und Anpassungsfähigkeit eher zur Stabilisierung der Konjunktur bei als Großunternehmen. Laut der „Krisenverstärkerhypothese“ verhält es sich hingegen genau umgekehrt, da in Zeiten des konjunkturellen Abschwungs die allgemeine Flucht in die Selbstständigkeit das Angebot des Handwerks - bei zugleich sinkender Nachfrage nach Handwerksleistungen - wachsen lassen würde. ${ }^{6}$ Im Ergebnis seiner Analyse leitet Schmidt für die Zeit von 1955 bis 1970 „die Tendenz verzögerter prozyklischer Reaktionen des Handwerks auf die Schwankungen der gesamtwirtschaftlichen Aktivität““7 ab.

Momm (1983) vertieft dieses Ergebnis durch eine Erweiterung des Untersuchungszeitraums auf die Jahre 1955 bis 1980. Gemessen an jährlichen Veränderungsraten wird dabei deutlich, dass das Gesamthandwerk und die Gesamtwirtschaft im Untersuchungszeitraum ein nahezu identisches Konjunkturmuster aufwiesen. Der Umsatz des Gesamthandwerks verlief über die Jahre hinweg im Gleichlauf zum Bruttosozialprodukt (entspricht dem Bruttonationaleinkom-

4 Der vorliegende Beitrag wurde im April 2010 abgeschlossen. Der Verfasser dankt Herrn Dr. Ullrich Kornhardt für wertvolle Anregungen und zahlreiche Diskussionen während der Entstehungszeit.

5 Vgl. Schmidt, K.-H. (1975); Momm, H.-J. (1983), S. 27ff und Dürig, W. u. a. (2004), S. 66ff.

$6 \quad$ Vgl. Schmidt, K.-H. (1975), S. 3.

7 Ebd., S. 42. 
men $^{8}$ ), wobei die Schwankungen des Handwerksumsatzes zum Teil größer ausfielen als die der gesamtwirtschaftlichen Produktion (vgl. Abbildung 1). Nach Momm verstärkt das Handwerk folglich eher Konjunkturschwankungen, „statt stabilisierenden Einfluss zu haben“9 .

Abbildung 1: Entwicklung der Gesamtwirtschaft und des Gesamthandwerks im Zeitraum 1955 bis 1980 (nominale Werte; jährliche Veränderungen)

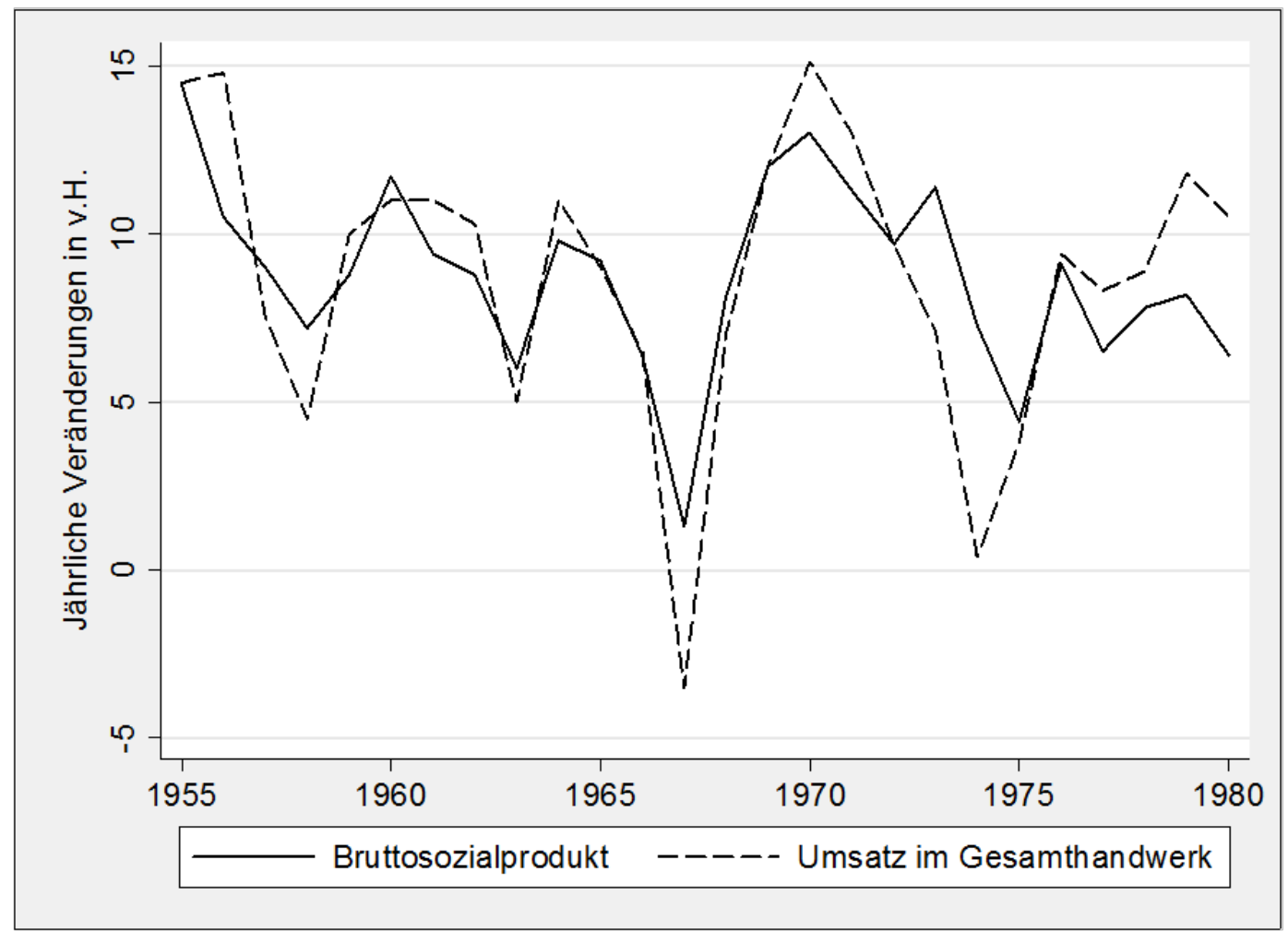

Quelle: Momm (1983), S. 50 und S. 256 (Tabelle 1)

Auch in der jüngeren Studie von Dürig u. a. (2004) setzen die Autoren an den Ergebnissen von Schmidt (1975) an. Der Vergleich der jährlichen Veränderungen des Bruttoinlandprodukts mit denjenigen des Umsatzes im westdeutschen Handwerk (Zeitraum 1950-2002) brachte hierbei wiederum das Resultat, dass sich das Handwerk im Zeitablauf eher prozyklisch verhält und die Schwankungen der Handwerksproduktion in der Regel sogar stärker ausgefallen sind als in der gesamtwirtschaftlichen Konjunktur. Insofern müsse nach Dürig u.a. nicht von einer konjunkturstabilisierenden, sondern von einer konjunkturverstärkenden Rolle der Handwerkswirtschaft ausgegangen werden. ${ }^{10}$ Im Vergleich zu früheren Untersuchungen erweitern die Autoren dieser Studie jedoch die Überprüfung der Stabilisatorhypothese um den Aspekt der Beschäftigungsentwicklung. Dies ist dem Umstand geschuldet, dass mit Blick auf den Arbeitsmarkt in der Vergangenheit regelmäßig eine stabilisierende Funktion des Hand-

8 Das Bruttonationaleinkommen (BNE) ist dem Bruttoinlandsprodukt (BIP) sehr ähnlich. Das BNE wird nach dem Inländerprinzip ermittelt. Das BIP wird nach dem Inlandsprinzip erhoben.

$9 \quad$ Momm, H.-J. (1983), S. 56.

10 Vgl. Dürig, W. u. a. (2004), S. 52ff und S. 66. 
werks angenommen wurde. In Handwerksbetrieben - so die entsprechende Annahme - wird demnach in Krisenzeiten tendenziell länger an Beschäftigten festgehalten als in Industriebetrieben. ${ }^{11}$

In der Tat lassen sich verschiedene Argumente anführen, die für eine vergleichsweise stabile Beschäftigungsentwicklung im Handwerk sprechen. Zunächst sind soziologische Argumente zu nennen, die von einem sozial motivierten Beschäftigungsverhalten ausgehen. Aufgrund der persönlichen Arbeitsbeziehungen in inhabergeführten Kleinbetrieben ist es demnach gerade für das Handwerk charakteristisch, dass Einstellungspraktiken eher auf eine dauerhafte Beschäftigung von Mitarbeitern abzielen und krisenbedingte Entlassungen nur als letzte Auswegmöglichkeit gesehen werden. Neben persönlichen Bindungen lässt sich aber vor allem eine Reihe von ökonomisch-rationalen Argumenten anführen, die für ein stabileres Beschäftigungsverhalten in Handwerksbetrieben sprechen. Die Einstellung neuer Mitarbeiter ist für Kleinbetriebe mit hohen Transaktions- und Einarbeitungskosten (Bearbeitung von Bewerbungen, Einstellungsgespräche, Aneignung betriebsspezifischen Wissens etc.) verbunden. Des Weiteren führt die wichtige Rolle des Humankapitals für die Handwerkswirtschaft nicht erst in Zeiten eines sich abzeichnenden Fachkräftemangels dazu, dass an qualifizierten Beschäftigten möglichst lange festgehalten wird. In diesem Zusammenhang ist zudem die hohe Bedeutung des betriebsspezifischen Erfahrungswissens für den Unternehmenserfolg im Handwerk hervorzuheben. Kündigungen in Handwerksbetrieben führen daher oftmals zu einem kritischen Know-how-Abfluss bzw. im Zuge des Stellenwechsels zu einem unerwünschten Wissenstransfer in andere Unternehmen. Nicht nur in Fällen, bei denen die Betriebe bereits in die berufliche Erstausbildung eines Beschäftigten investiert haben, wird daher auch in Krisenzeiten möglichst lange an qualifizierten Fachkräften festgehalten. Zudem ist die kleinbetrieblich geprägte Größenstruktur des Handwerks als zentraler Faktor zu sehen. Beschäftigtenverluste sind in Kleinunternehmen weniger gut zu verkraften als in Großunternehmen, da z.B. durch die Entlassung eines Mitarbeiters in einem 5-Personen-Betrieb eine deutlich größere Lücke in der Personal- und Organisationsstruktur entsteht als in einem 100-Personen-Betrieb. $^{12}$

Dürig u. a. (2004) sehen insbesondere in den genannten ökonomischen Argumenten eine Ursache für den oftmals zu beobachtenden Umstand, dass viele Handwerksbetriebe in konjunkturellen Abschwungphasen an ihren Beschäftigten möglichst lange festhalten. Dennoch bezweifeln Dürig u.a. eine sich hieraus ergebende Stabilisatorfunktion des Handwerks für die Gesamtwirtschaft, da sie vermuten, dass im konjunkturellen Verlauf ein konservatives Beschäftigungsverhalten von einzelnen Handwerksbetrieben nicht zu einer Stabilisierung der handwerklichen Gesamtbeschäftigung führt. ${ }^{13}$ Verantwortlich hierfür wäre der prozyklische

\footnotetext{
11 Vgl. Dispan, J. (2003), S. 120.

12 Vgl. Dürig, W. u. a. (2004), S. 68ff.

13 Vgl. ebd., S. 70f.
} 
Aggregationseffekt der kontinuierlichen Personalfluktuation (Austausch von Beschäftigten im Zuge von Personalzugängen und -abgängen), welcher in Kleinunternehmen erfahrungsgemäß deutlich größeren Schwankungen unterliegen würde als in Großunternehmen. ${ }^{14}$ In der Summe - so die These der Autoren - führt das konservative Beschäftigungsverhalten der handwerklichen Kleinbetriebe dazu, „dass in Rezessionszeiten Neueinstellungen bei entstehenden Vakanzen zögerlicher erfolgen und in Aufschwungphasen - wenn die Auftragslage besser ist umso bereitwilliger. “15 Zur Bekräftigung ihrer These vergleichen Dürig u.a. die jährlichen Veränderungen des Beschäftigungswachstums im westdeutschen Handwerk mit derjenigen der gesamtwirtschaftlichen Erwerbstätigkeit (Zeitraum 1950 bis 2002). Ähnlich zum obigen Fall der handwerklichen Produktion kommen die Autoren zu dem Schluss, dass sich die Beschäftigung im Gesamthandwerk eher prozyklisch verhalten hat und das Handwerk daher im Zeitverlauf auch kein Beschäftigungsstabilisator gewesen ist. ${ }^{16}$

Der Überblick zu den bisherigen Forschungsarbeiten spricht folglich eher gegen die Gültigkeit der Stabilisatorhypothese. Die vorliegenden Ergebnisse sprechen sogar dafür, dass dem Handwerk anstatt einer stabilisierenden eher eine konjunkturverstärkende Funktion zukommt. Gerade im Falle der jüngsten Wirtschaftskrise wurde der Handwerkswirtschaft jedoch wiederholt eine konjunkturelle Stabilisatorfunktion zugeschrieben. ${ }^{17}$ In den folgenden Kapiteln wird daher untersucht, ob die Verneinung einer konjunkturellen Stabilisatorfunktion des Handwerks nicht dennoch einer gewissen Differenzierung bedarf. Hierzu wird in Kapitel 3 überprüft, ob das Handwerk in der Krise 2008/2009 trotz des relativ starken Rückgangs des Handwerksumsatzes im Jahr 2009 als Konjunkturstabilisator gewirkt hat. Des Weiteren wird der Frage nachgegangen, was über die aktuellen Ereignisse hinaus für eine generelle Gültigkeit der Stabilisatorhypothese sprechen könnte. Die oben angeführten Forschungsarbeiten liefern hierfür einen wichtigen Ausgangspunkt. Dennoch sollen deren Ergebnisse in den Kapitel 5 und 6 aus den folgenden Gründen vertieft bzw. ergänzt werden: Erstens fällt auf, dass die Arbeiten zur Überprüfung der Stabilisatorhypothese das Gesamthandwerk als Untersuchungseinheit gewählt haben. Gerade das Handwerk ist jedoch keine homogene Branche gemäß der

14 Die Ergebnisse von Müller, K. (2005) deuten darauf hin, dass dies nicht uneingeschränkt gilt. Für die Gesamtwirtschaft konnte dabei auf Grundlage des IAB-Betriebspanels zwar in der Tat eine steigende Fluktuationsrate mit sinkender Betriebsgröße festgestellt werden. Im Falle des Handwerks verhielt es sich hingegen genau umgekehrt, d.h. die Fluktuationsrate stieg mit der Betriebsgröße an. Hierbei lag die Fluktuation im Handwerk in den unteren Betriebsgrößenklassen in etwa auf dem Niveau der Gesamtwirtschaft. Vgl. Müller, K. (2005), S. 49 und S. 107.

15 Dürig, W. u. a. (2004), S. 71.

16 Vgl. ebd., S. 54 und S. 71.

17 Vgl. z.B. IW-Köln (2010). In der öffentlichen Diskussion zur Wirtschaftskrise wurde die stabilisierende Wirkung des Handwerks wiederholt hervorgehoben. Vgl. z.B. die folgenden Artikel aus der Handwerkspresse: "Handwerk trotzt der Wirtschaftskrise“, in: Allgemeine Fleischer-Zeitung Nr. 45 vom 04.11.2009, S. 9; "Handwerk in Rezession weitgehend robust", in: Allgemeine Bäcker-Zeitung Nr. 01 vom 16.01.2010, S. 28; "Handwerk als stabilisierender Faktor“, in: Deutsche Handwerkszeitung, Ausgabe HWK Wiesbaden vom 18.12.2009, S. 1. 
Wirtschaftszweigsystematik. ${ }^{18}$ Diese Heterogenität sollte daher auch in einer Untersuchung zur etwaigen Stabilisatorfunktion des Handwerks durch eine getrennte Betrachtung von handwerklichen Gewerbegruppen berücksichtigt werden. Zweitens liegen den bereits vorliegenden Untersuchungen jährliche Werte zur Messung des langfristigen Konjunkturverlaufs im Handwerk zu Grunde. Zur Analyse von eher kurzfristigen Konjunkturschwankungen sind jedoch gerade Quartalsdaten von Interesse, da sie die Datengrundlage um wichtige unterjährige Informationen erweitern. ${ }^{19}$ Der Analyse in den Kapiteln 5 und 6 liegen daher vierteljährliche Daten über die Beschäftigungs- und Umsatzentwicklung im Handwerk zu Grunde. ${ }^{20}$ Drittens setzt eine Beantwortung der Ausgangsfrage zumindest näherungsweise das Aufspüren von konjunkturellen Zyklen im Handwerk durch einen Ausschluss von anderweitigen Einflüssen voraus. Solche Einflüsse können saisonaler Natur sein oder eher mit längerfristigen Trendentwicklungen zusammenhängen, wie z.B. den strukturell bedingten Beschäftigungs- und Umsatzverlusten im Handwerk seit der Mitte der 90er Jahre. ${ }^{21}$

\section{Die Krise 2008/2009: das Handwerk als Stabilitätsanker?}

\subsection{Lage der Gesamtwirtschaft und wirtschaftspolitische Reaktionen}

Im Winterhalbjahr 2008/2009 griff die weltweite Nachfrageschwäche in Folge der anfänglichen Finanzmarktkrise auch auf die deutsche Wirtschaft über. In der Folge stand in der Bilanz des Krisenjahres 2009 mit einer Schrumpfung des realen Bruttoinlandsprodukts um $5 \%$ gegenüber dem Vorjahr die bis dato schwerste Rezession in der bundesdeutschen Wirtschaftsgeschichte zu Buche. Der Konjunkturabschwung hatte sich zuvor bereits seit einiger Zeit angekündigt. Hierbei verlief die Entwicklung bis zum August 2008 zunächst noch ähnlich wie in den vorangegangenen Abschwungphasen der Jahre 1992 und 2001. Durch die Insolvenz der Investmentbank Lehman Brothers im September 2008 kulminierte jedoch nicht nur das Misstrauen an den Weltfinanzmärkten, wodurch der Interbankenmarkt zum Erliegen kam und weltweit die Zentralbanken zur Sicherstellung der Liquiditätsversorgung einspringen mussten. Die durch eine starke psychologische Komponente geprägte Finanzkrise schlug nun auch realwirtschaftlich durch, was sich z.B. in verschlechterten Bedingungen für die Unternehmensfinanzierung äußerte. Vor allem im internationalen Wettbewerb stehende Industrieunternehmen waren hiervon in hohem Maße betroffen, was sich in massiven Auftrags- und Produktionseinbrüchen äußerte. Das Welthandelsvolumen ging zwischen November 2008 und Januar 2009 um fast 20 \% zurück. Die starke internationale Verflechtung von Branchen und Wertschöpfungsketten hat somit erheblich zur weltweiten Verbreitung der zunächst nur auf die USA beschränkten Nachfrageschwäche beigetragen. Aufgrund ihrer hohen Export- und In-

\footnotetext{
18 Vgl. Kornhardt, U. und Kucera, G. (2003), S. 108ff.

19 Vgl. Schirwitz, B. u. a. (2008), S. $18 f$.

20 Zur Datenquelle vgl. Kapitel 4.

21 Vgl. Lageman, B. u. a. (2005), S. 4ff.
} 
dustrieorientierung war Deutschland hiervon in stärkerem Maße betroffen als viele andere fortgeschrittene Volkswirtschaften. ${ }^{22}$

Abbildung 2: Entwicklung ausgewählter BIP-Verwendungskomponenten (saison- und preisbereinigt; 2000 bis 2009; Kettenindex; 2000 = 100)

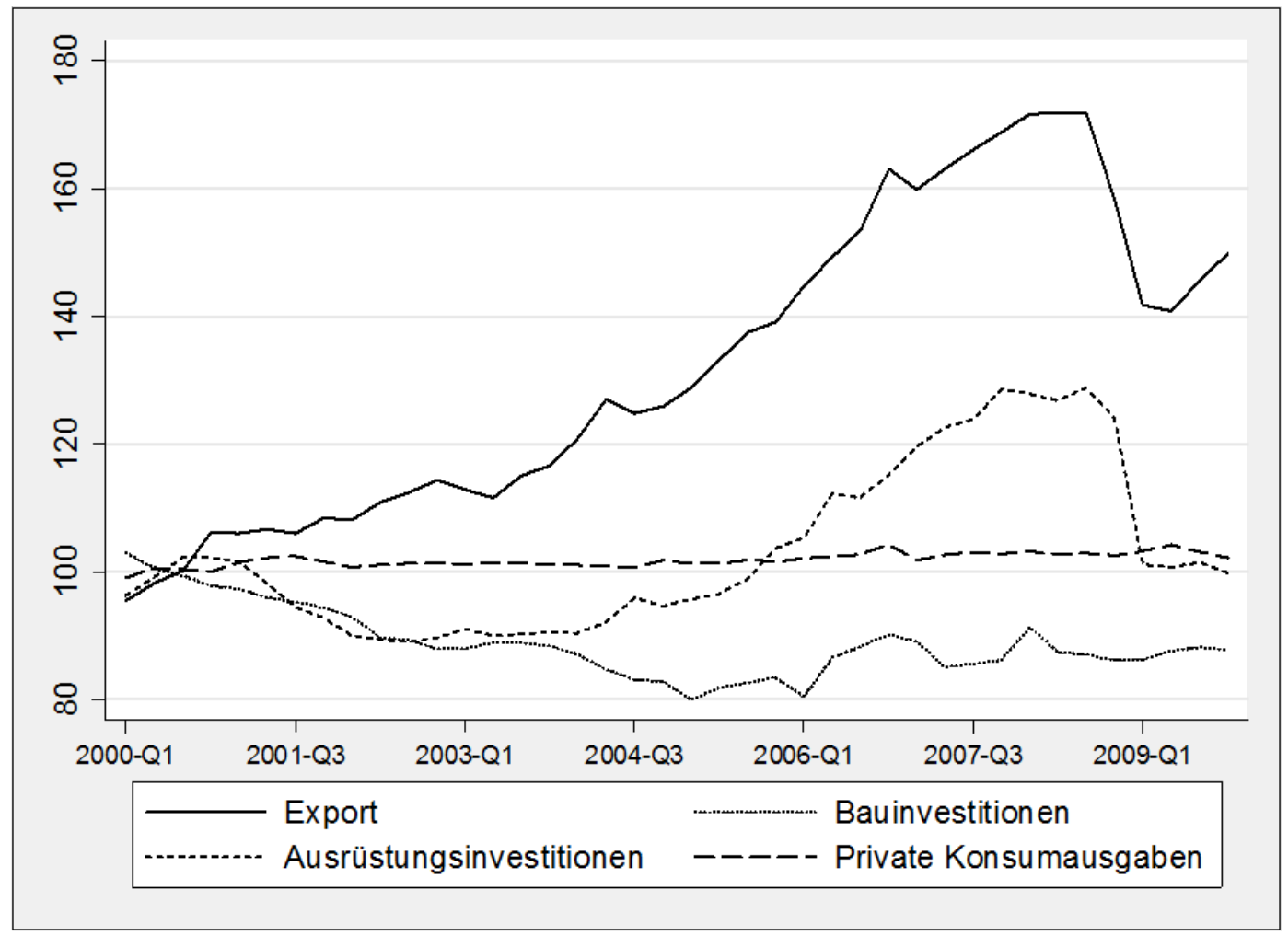

Quelle: Sachverständigenrat

Die Entwicklung der deutschen Wirtschaft im Krisenjahr 2009 kann dabei (vereinfacht) am Verlauf von einzelnen Verwendungskomponenten ${ }^{23}$ des Bruttoinlandsprodukts abgelesen werden (vgl. Abbildung 2). ${ }^{24}$ War der Export im vergangenen Konjunkturzyklus noch die treibende Wachstumskraft gewesen, bremste er im Jahr 2009 die gesamtwirtschaftliche Entwicklung deutlich. Der Exporteinbruch beendete damit (vorerst) das prägende Wachstumsmuster der vorangegangenen Jahre: Erstmals seit 1993 lag in Deutschland die reale Ausfuhr an Waren und Dienstleistungen unter dem Wert des Vorjahres. Da sich in der deutschen Volkswirtschaft das Exportgeschäft zu fast 90 \% aus Gütern zusammensetzt, wurden Unternehmen des Verarbeitenden Gewerbes und hierbei vor allem diejenigen der exportorientierten Industrie besonders stark in Mitleidenschaft gezogen. Im Jahresverlauf setzte zwar wieder eine

22 Vgl. IW-Köln (2009), S. $15 f$ und ifo (2009a), S. 3f.

23 Die Volkswirtschaftliche Gesamtrechnung setzt zur Ermittlung des Bruttoinlandsprodukts u.a. auf der Nachfrageseite an (Verwendungsrechnung). Das Bruttoinlandsprodukt ergibt sich hier aus der Summe der privaten Konsumausgaben, der Konsumausgaben des Staates, der Bruttoinvestitionen (einschließlich Vorratsveränderungen) und des Außenbeitrags (Exporte abzüglich Importe).

24 Zur Lage der deutschen Volkswirtschaft im Jahr 2009 vgl. im Folgenden auch ifo (2009a), S. 29ff und ifo (2009b), S. 27ff. 
allmähliche Erholung der Exporte ein; gegenüber dem Vorjahr sanken die deutschen Ausfuhren im Jahr 2009 dennoch preisbereinigt um 14,2 \% (nominal:-16,7 \%). ${ }^{25}$

Mit dem Krisenjahr 2009 endete zudem die hohe Investitionsdynamik der vorangegangenen drei Jahre. Die Bruttoinvestitionen, welche sich gemäß der Volkswirtschaftlichen Gesamtrechnung aus den Bruttoanlageinvestitionen (vorrangig Ausrüstungsinvestitionen und Bauinvestitionen) und den Vorratsänderungen zusammensetzen, sanken preisbereinigt um 15,7 \%. Dieser Rückgang war in erster Linie auf den Einbruch der Ausrüstungsnachfrage zurückzuführen: Im Vergleich zum Vorjahr investierten die Unternehmen sowohl nach realer (-20,5 \%) als auch nach nominaler Rechnung (-22\%) deutlich weniger in Ausrüstungsgüter, worunter z.B. Maschinen, Geräte (einschließlich Ausstattungen) oder Fahrzeuge fallen. Die Unternehmen haben somit auf den negativen Nachfrageschock der Krise durch eine rasche Anpassung ihrer Investitionspläne reagiert. An diesem Beispiel zeigt sich sehr gut, dass es sich bei den Ausrüstungsinvestitionen in der Regel um die konjunktursensibelste Verwendungskomponente des Bruttoinlandsprodukts handelt. Angesichts des eingetrübten Investitionsklimas waren die gewerblichen Bauinvestitionen ebenfalls stark von der Rezession betroffen. In der Summe blieb die Baunachfrage jedoch vergleichsweise stabil, da im Jahr 2009 bei den Bauinvestitionen nur ein leichter preisbereinigter Rückgang gegenüber $2008 \mathrm{zu}$ verzeichnen war (-0,8 \%). Nominal gesehen blieben die Bauinvestitionen nahezu auf dem Vorjahresniveau ( $+0,1 \%)$. Der private Konsum wirkte im Krisenjahr 2009 ebenfalls stabilisierend. Von ihm gingen sogar leicht positive Impulse aus, da gegenüber dem Jahr 2008 die Konsumausgaben der privaten Haushalte preisbereinigt um 0,17 \% bzw. nominal um 0,18 \% zulegten. Einen wichtigen Beitrag zu diesem positiven Jahresergebnis lieferten die durch die sog. „Abwrackprämie“ angeregten privaten Kraftfahrzeugkäufe. $^{26}$

Die „Abwrackprämie“ war zuvor im Rahmen des deutschen Konjunkturprogramms von der Bundesregierung beschlossen worden. Neben einer expansiven Ausrichtung der Geldpolitik und weiterer Maßnahmen zur Stabilisierung der Finanzmärkte wurden im Zuge der Finanzund Wirtschaftskrise weltweit diskretionäre Konjunkturprogramme aufgelegt, um die rezessionsbedingten Folgen für die Realwirtschaft abzufedern. Die hiermit verbundenen fiskalpolitischen Maßnahmen umfassen grundsätzlich alle Möglichkeiten eines Staates, öffentliche Ausgaben und Einnahmen für Zwecke der Konjunkturpolitik anzupassen. ${ }^{27}$ Nach anfäng-

25 Vgl. IW-Köln (2009), S 27f; Statistisches Bundesamt (2010), S. 178; Statistisches Bundesamt, Pressemitteilung Nr. 012 vom 13.01.2010, „Deutschland erlebte 2009 schwerste Rezession der Nachkriegszeit“, in: http://www.destatis.de (letzter Zugriff: 30.03.2010).

26 Vgl. IW-Köln (2009), S. 30 ff; Statistisches Bundesamt (2010), S. 178; Statistisches Bundesamt, Pressemitteilung Nr. 012 vom 13.01.2010, „Deutschland erlebte 2009 schwerste Rezession der Nachkriegszeit“, in: http://www.destatis.de (letzter Zugriff: 30.03.2010).

27 Unabhängig hiervon haben in der Krise die automatischen Stabilisatoren eine starke Wirkung entfaltet. Dabei handelt es sich um bestimmte Staatsausgaben bzw. -einnahmen, die naturgemäß mit der Konjunktur schwanken und somit von sich aus eine stabilisierende Wirkung entfalten können (z.B. die Arbeitslosenversicherung oder die progressive Ausgestaltung der Einkommenssteuer). 
lichem Zögern schlug die Bundesregierung diesen Weg mit dem Beschluss der Konjunkturpakete I (5.11.2008) und II (27.1.2009) sowie weiterer nachfrageorientierter Impulse in einem Gesamtumfang von ca. 85 Mrd. Euro ein. Auch im Handwerk boten sich hierdurch eine Reihe von Chancen (vgl. Tabelle 1). ${ }^{28}$

Tabelle 1: Handwerksrelevante Teile der Konjunkturpakete

\begin{tabular}{|c|c|}
\hline Konjunkturpaket I & Konjunkturpaket II \\
\hline $\begin{array}{l}\text { - Sonderkreditprogramm der KfW im Umfang } \\
\text { von } 15 \text { Mrd. Euro für kleine und mittlere } \\
\text { Unternehmen zur Vermeidung einer Kredit- } \\
\text { klemme im Mittelstand } \\
\text { - Erhöhte Absetzbarkeit von Handwerker- } \\
\text { leistungen durch Verdoppelung des } \\
\text { Steuerbonus } \\
\text { - } \quad \text { Aufstockung der Fördermittel für ener- } \\
\text { getische Gebäudesanierung und ener- } \\
\text { gieeffizientes Bauen um } 3 \text { Mrd. Euro } \\
\text { - Aufstockung der Infrastrukturprogramme für } \\
\text { Kommunen und Verkehrsinvestitionen sowie } \\
\text { der Gemeinschaftsaufgabe regionale Wirt- } \\
\text { schaftstruktur um über } 5 \text { Mrd. Euro. } \\
\text { Beschleunigung der Verfahren } \\
\text { - Aufstockung der KfW-Innovationsförderung } \\
\text { (ERP-Innovationsprogramm, ERP-Start- } \\
\text { fonds, Sonderfonds Energieeffizienz) } \\
\text { Verlängerung der Bezugsdauer von } \\
\text { Kurzarbeitergeld } \\
\text { Wiedereinführung der degressiven Ab- } \\
\text { schreibung für bewegliche Wirtschaftsgüter } \\
\text { des Anlagevermögens i. H. v. 25 Prozent } \\
\text { sowie Erweiterung der Sonderabschrei- } \\
\text { bungen und von Investitionsabzugsbeträgen } \\
\text { für kleine und mittlere Unternehmen } \\
\text { Stützung der Automobilindustrie und des } \\
\text { Kfz-Gewerbes durch Einführung einer Um- } \\
\text { weltprämie (,Abwrackprämie“), befristete } \\
\text { Kfz-Steuerbefreiung für Neuwagen und } \\
\text { schadstoffbezogene Umstellung der Kfz- } \\
\text { Steuer }\end{array}$ & $\begin{array}{l}\text { - Senkung der einkommenssteuerlichen Belas- } \\
\text { tung (Anhebung des Grundfreibetrags auf } \\
\text { 7.834 Euro und Rechtsverschiebung der Tarif- } \\
\text { eckwerte um } 400 \text { Euro) als Vorteil für Einzel- } \\
\text { unternehmer und Personengesellschaften im } \\
\text { Handwerk } \\
\text { - Stabilisierung der Lohnzusatzkosten durch } \\
\text { Senkung des paritätisch finanzierten Beitrags- } \\
\text { satzes zur gesetzlichen Krankenversicherung } \\
\text { um 0,6 Prozentpunkte sowie durch eine } \\
\text { Beitragssatzgarantie in der } \\
\text { Arbeitslosenversicherung } \\
\text { - Zusätzliche Investitionen der öffentlichen } \\
\text { Hand in Infrastruktur und Bildung von ins- } \\
\text { gesamt } 14 \text { Mrd. Euro; vereinfachtes Vergabe- } \\
\text { recht zur Beschleunigung von Investitionen } \\
\text { - Flexibilisierung des KfW-Sonderprogramms } \\
\text { für den Mittelstand. Einrichtung eines zusätz- } \\
\text { lichen Kredit- und Bürgschaftsprogramms im } \\
\text { Volumen von } 100 \text { Mrd. Euro. Prüfung staat- } \\
\text { licher Unterstützung im Bereich Warenkredit- } \\
\text { versicherung, Exportgarantien, Leasing und } \\
\text { Factoring } \\
\text { - Nochmalige Aufstockung des Zentralen Inno- } \\
\text { vationsprogramms Mittelstand (ZIM) um } 450 \\
\text { Mio. Euro und Ausweitung der Förderung auf } \\
\text { ganz Deutschland sowie mittelgroße } \\
\text { Unternehmen } \\
\text { - Weitere Verlängerung und Erleichterung des } \\
\text { Kurzarbeitergeldes u.a. durch Übernahme der } \\
\text { vollständigen Sozialbeiträge ab dem siebten } \\
\text { Monat } \\
\text { - Stärkere Förderung der beruflichen Wei- } \\
\text { terbildung von Beschäftigten } \\
\text { - Aufstockung der Mittel für die „Abwrack- } \\
\text { prämie“ }\end{array}$ \\
\hline
\end{tabular}

Quelle: PKM (2009), S. 16. 
Obwohl eine Skepsis hinsichtlich der grundsätzlichen Tauglichkeit von fiskalpolitischen Konjunkturprogrammen stark verbreitet ist herrscht unter Experten weitgehend Einigkeit darüber, dass im Fall der Wirtschaftskrise 2008/2009 angesichts des globalen Ausmaßes der realwirtschaftlichen Krisenerscheinungen und der gestörten Wirksamkeit der Geldpolitik in Folge der Finanzkrise eine aktive Fiskalpolitik notwendig war. Trotz vorhandener Unsicherheiten hinsichtlich der Effektivität von einzelnen fiskalpolitischen Maßnahmen und den zukünftigen Herausforderungen der unter anderem hierdurch gestiegenen Staatsverschuldung kam der Sachverständigenrat zur Begutachtung der gesamtwirtschaftlichen Entwicklung daher zu dem Schluss, dass „die Konjunkturprogramme die gesamtwirtschaftliche Nachfrage stabilisiert und einen noch stärkeren Einbruch des Bruttoinlandsprodukts verhindert [haben]. Ohne sie wäre alles noch schlimmer gekommen.“29

\subsection{Betroffenheit des Handwerks}

Auch die Handwerkskonjunktur konnte sich im Winterhalbjahr 2008/2009 der weltweiten Krise nicht entziehen. Der konjunkturelle Abschwung hatte sich dabei insbesondere im 1. Quartal 2009 verschärft, wobei jedoch kein Einbruch wie in anderen Bereichen der deutschen Wirtschaft zu verzeichnen war. In der Folge rutschte die Stimmung der Handwerksbetriebe im Frühjahr 2009 zwar spürbar ab. Nur noch 64\% der Betriebsinhaber beurteilten ihre Geschäftslage als zufriedenstellend oder gut, was im Vergleich zum Vorjahresquartal einem Rückgang um 9 Prozentpunkte entsprach. Diese Eintrübung der Stimmungslage im Handwerk fiel jedoch deutlich schwächer aus als in der gewerblichen Wirtschaft (vgl. Abbildung 3). Trotz des konjunkturellen Rückschlags blieb damit die (gefühlte) Wirtschaftslage im Handwerk - auch an den Rückgängen anderer Konjunkturindikatoren gemessen - vergleichsweise stabil. $^{30}$

Im 1. Quartal 2009 hatte sich dennoch die wirtschaftliche Lage in allen Gewerbegruppen des Handwerks verschlechtert. Die Wirtschaftskrise traf dabei einige Teile des Handwerks und hierbei in erster Linie die handwerklichen Zulieferer der Industrie besonders hart. Die weggebrochene Auslandsnachfrage und der freie Fall der Industrieproduktion hatten in diesem Handwerksbereich mit einer gewissen zeitlichen Verzögerung zu einem massiven Nachfragerückgang geführt. In Folge eines langen und harten Winters verschlechterte sich zudem die Entwicklung der Bauhandwerke neben konjunkturellen Gründen auch aufgrund von besonders widrigen saisonalen Umständen. Aus konjunktureller Sicht hatte die Wirtschaftskrise in diesem Handwerksbereich vor allem zu einem starken Rückgang der Nachfrage nach gewerblichen Bauten geführt. Vergleichsweise positiv stellte sich die Lage in den Ausbauhandwerken dar, was auf eine fortwährend hohe Nachfrage nach Sanierungsmaßnahmen im Bestand und energiesparende Maßnahmen zurückzuführen war. Auch in den konsumnahen

29 Sachverständigenrat (2009), S. 166.

30 Vgl. ZDH (2009a), S. 7ff. 
Gewerbegruppen blieb am Anfang des Krisenjahrs 2009 die Lage weitgehend stabil. Im KfzHandwerk hatte die Einführung der „Abwrackprämie" im Rahmen der Konjunkturpakete zu einer spürbaren Belebung der Nachfrage nach Kleinwagen geführt. Absatzprobleme in den Mittel- und Oberklassesegmenten und ein nachlassendes Werkstattgeschäft bremsten jedoch hier die Entwicklung. ${ }^{31}$

Abbildung 3: Geschäftslage im Handwerk im Vergleich zur gewerblichen Wirtschaft (Werte zum 1. und 3. Quartal; Salden; saisonbereinigt)

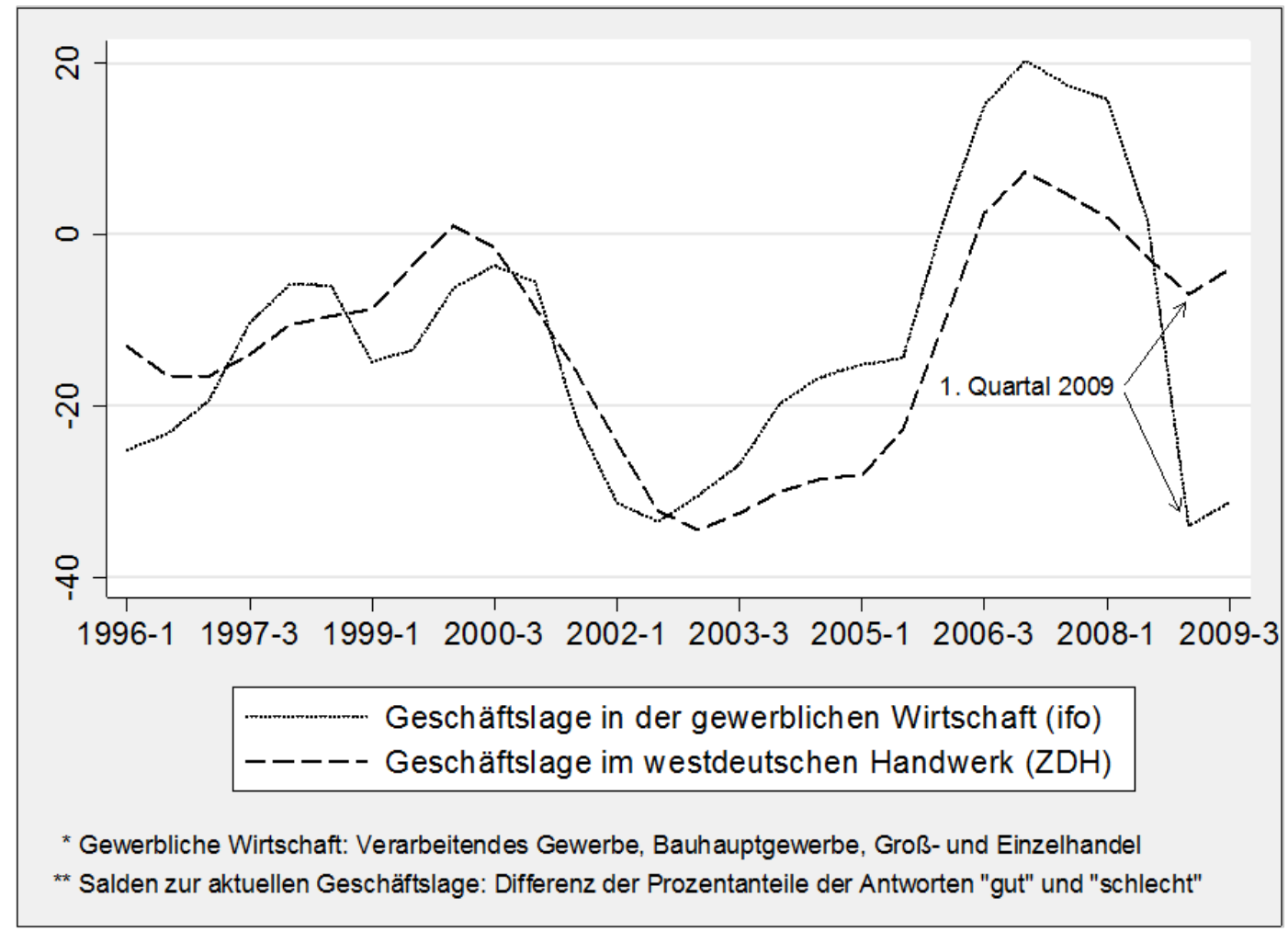

Quelle: ifo-Institut, Zentralverband des Deutschen Handwerks, eigene Berechnungen

Im Laufe des Krisenjahrs 2009 belebte sich die Handwerkskonjunktur spürbar. Im 3. Quartal 2009 zeigte sich die Stimmung in den Handwerksbetrieben deutlich aufgehellt (vgl. Abbildung 3). Nun beurteilten 76 \% der befragten Handwerksbetriebe ihre Geschäftslage als zufriedenstellend oder gut. Im Vergleich zum krisengeschüttelten 1. Quartal entsprach dies einer Verbesserung um 12 Prozentpunkte. Diese Entwicklung basierte neben günstigen saisonalen Effekten auch auf den anregenden Wirkungen der Konjunkturpakete im Bereich der Bauwirtschaft und der privaten Konsumnachfrage. Zugleich wirkten jedoch die Folgen der gesunkenen Industrie- und Exportnachfrage in den hiervon betroffenen Handwerksbereichen weiterhin deutlich nach. Hierdurch erklärt sich der Umstand, dass innerhalb der Handwerkskonjunktur im Zuge der Wirtschaftskrise 2008/2009 fortwährend starke branchenspezifische

31 Vgl. ebd., S. 7f. Siehe auch Creditreform (2009). 
Unterschiede bestanden haben. In der Summe hatte sich die Handwerkswirtschaft jedoch am Ende des Krisenjahrs 2009 wieder gefestigt. ${ }^{32}$

Im Zuge der skizzierten Entwicklung musste sich im Frühjahr 2009 ein größerer Anteil der Betriebsinhaber von Mitarbeitern trennen als noch im Vorjahresquartal (I/2009: 20 \% vs. I/2008: 16 \%). Zugleich konnten weniger Betriebe zusätzliche Mitarbeiter einstellen (I/2009: 7 \% vs. I/2008: $10 \%$ ). Eine Reihe von Inhabern sah sich folglich im 1. Quartal 2009 gezwungen, auf die krisenbedingten Nachfragerückgänge mit einem Beschäftigungsabbau zu reagieren. Dennoch hatten damit aber immerhin noch 80 \% der Handwerksbetriebe ihren Beschäftigungsstand zumindest stabil halten können. Die Handwerkskonjunktur war auch in diesem Fall in sich sehr heterogen. Vor allem die krisengeschüttelten Zulieferer der Industrie mussten im Frühjahr 2009 Mitarbeiter entlassen. 23 \% der entsprechenden Betriebe verzeichneten einen Beschäftigungsabbau (I/2008: $12 \%$ ), während nur noch 8 \% zusätzliche Mitarbeiter einstellen konnten (I/2008: 16 \%). Vor dem Hintergrund der aufgehellten Konjunkturlage stabilisierte sich dann auch die Handwerksbeschäftigung in der Summe wieder im 3. Quartal des Jahres 2009. Viele Handwerksunternehmer hatten nicht nur versucht, so lange wie möglich an ihren Fachkräften festzuhalten, sondern stellten sogar nach Möglichkeit zusätzliche Mitarbeiter ein. Hierbei war jedoch ebenfalls zu beobachten, dass das positive Gesamtergebnis vom Herbst 2009 die immer noch angespannte Beschäftigungslage in einigen Handwerksbereichen (v.a. bei den handwerklichen Zulieferern) überdeckte. ${ }^{33}$

Trotz der belebten Handwerkskonjunktur im Jahresverlauf und der im Vergleich zur Gesamtwirtschaft in vielen Handwerksbetrieben stabil gebliebenen Geschäftslage fällt die Bilanz zum Krisenjahr 2009 aus Sicht des Handwerks - in erster Linie aufgrund des Einbruchs der handwerklichen Zulieferer - kritisch aus (vgl. Tabelle 2). Im Gesamtjahr 2009 verzeichneten die zulassungspflichtigen Handwerke im Vergleich zum Vorjahr einen Rückgang ihrer nominalen Umsätze um $-5 \%{ }^{34}$ Prozentual gesehen fiel dieser Ausschlag somit stärker aus als im Falle des nominalen Bruttoinlandsprodukts (-3,5\%). Daher liegt zunächst der Schluss nahe, dass das Handwerk in der Wirtschaftskrise 2008/2009 nicht als Konjunkturstabilisator gewirkt hat.

Die mit Abstand stärksten Umsatzeinbußen im Handwerk meldeten dabei die industrienahen Handwerke für den gewerblichen Bedarf, worunter die handwerklichen Zulieferer fallen ($17,7 \%)$. Im Vergleich hierzu deutlich schwächer, aber prozentual gesehen dennoch relativ stark gesunken sind zudem die Umsätze im Bauhauptgewerbe (-6,9 \%). Im Jahresverlauf hatte sich die konjunkturelle Lage in dieser Gewerbegruppe zwar zunehmend verbessert, zudem entfaltete das kommunale Investitionsprogramm als Teil des Konjunkturprogramms ab Herbst 2009 erste stimulierende Wirkungen. Der krisenbedingte Nachfrageeinbruch im Wirtschaftsbau schlug sich dennoch deutlich in der Jahresbilanz des Bauhauptgewerbes nieder. In den

\footnotetext{
32 Vgl. ZDH (2009b), S. 7f und ZDH (2009c).

33 Vgl. ZDH (2009a), S. 8f und S. 14f; ZDH (2009b), S. 8f und S. 14f.

34 Zugleich ist die Beschäftigung im zulassungspflichtigen Handwerk um -1,5 \% gesunken.
} 
Ausbauhandwerken fiel das Krisenjahr 2009 ebenfalls negativ aus (3,3 \% weniger Umsätze als im Vorjahr). Dieses Ergebnis musste überraschen, da die Betriebe des Ausbaugewerbes in den Konjunkturumfragen des Jahres 2009 ihre Stimmungslage durchweg besser eingeschätzt hatten als im übrigen Handwerk. In den anderen Gewerbegruppen des Handwerks verlief die Umsatzentwicklung hingegen deutlich stabiler, wozu nicht zuletzt die anregenden Wirkungen der Konjunkturpakete auf die Konsumbereitschaft der privaten Verbraucher beigetragen haben. Das Kraftfahrzeuggewerbe erzielte im Jahr 2009 aufgrund der „Abwrackprämie“ ein Umsatzwachstum von $+1,7$ \%. Im Lebensmittelgewerbe war ein leichter Umsatzrückgang um $-1,2 \%$ zu verbuchen, die Gesundheitshandwerke legten leicht zu (+0,9 \%) und bei den personenbezogenen Dienstleistungshandwerken stagnierte die Umsatzentwicklung auf dem Vorjahresniveau. ${ }^{35}$

Tabelle 2: Umsatzentwicklung im zulassungspflichtigen Handwerk - Bilanz 2009

\begin{tabular}{|l|c|}
\hline & $\begin{array}{c}\text { Veränderung der Umsätze im Jahr } \\
2009 \text { gegenüber 2008 in \% }\end{array}$ \\
\hline Zulassungspflichtiges Handwerk insgesamt & $-5,0$ \\
\hline davon folgende Gewerbegruppen: & $-6,9$ \\
Bauhauptgewerbe & $-3,3$ \\
Ausbaugewerbe & $-17,7$ \\
Handwerke für den gewerblichen Bedarf & 1,7 \\
Kraftfahrzeuggewerbe & $-1,2$ \\
Lebensmittelgewerbe & 0,9 \\
Gesundheitsgewerbe & 0,0 \\
Personenbezogene Dienstleistungsgewerbe & \\
\hline
\end{tabular}

Quelle: Statistisches Bundesamt, Pressemitteilung Nr. 110 vom 19.03.2010, „5,0 \% weniger Umsatz im Handwerk im Jahr 2009“, in: http://www.destatis.de (letzter Zugriff: 27.04.2010).

Die bisherigen Ausführungen scheinen einen Widerspruch in sich zu bergen: Die laut den Konjunkturumfragen in der Wirtschaftskrise 2008/2009 insgesamt vergleichsweise stabil gebliebene Geschäftslage des Handwerks spiegelt sich nicht im oben genannten Gesamtergebnis zur jährlichen Umsatzentwicklung im Handwerk wider. Denn demzufolge hat das Handwerk im Vergleich zur Gesamtwirtschaft keineswegs als Stabilisator wirken können. Zwei Gründe sprechen dennoch dafür, dass zumindest vom überwiegenden Teil des Handwerks im Krisenjahr 2009 eine stabilisierende Wirkung ausgegangen ist.

35 Vgl. Statistisches Bundesamt, Pressemitteilung Nr. 110 vom 19.03.2010, „5,0 \% weniger Umsatz im Handwerk im Jahr 2009“, in: http://www.destatis.de (letzter Zugriff: 27.04.2010). Siehe auch ZDH (2009a), S. 7 ff; ZDH (2009b), S. 7ff und ZDH (2009c). 
Erstens wäre nach Schätzungen des Zentralverbandes des Deutschen Handwerks (ZDH) ohne die krisengeschüttelten Handwerke für den gewerblichen Bedarf die Jahresbilanz 2009 mit einem nominalen Rückgang des Handwerksumsatzes in Höhe von etwa -2 \% deutlich geringer ausgefallen. Zweitens ist zu beachten, dass der starke nominale Umsatzrückgang im Handwerk von -5 \% gegenüber dem Jahr 2008 neben eher kurzfristigen konjunkturellen Faktoren auch längerfristigen strukturellen Einflüssen geschuldet sein dürfte. Denn gerade im Handwerk wird die konjunkturelle Entwicklung seit geraumer Zeit durch starke strukturelle Anpassungsprozesse überlagert. ${ }^{36}$ Die Antwort auf die Frage, ob das Handwerk in der Wirtschaftskrise 2008/2009 als Konjunkturstabilisator gewirkt hat oder nicht, setzt daher zumindest näherungsweise eine Verdeutlichung der tatsächlichen Konjunkturzyklen im Handwerk durch einen Ausschluss von längerfristigen Entwicklungstrends voraus. In diesem Fall wäre dann von einer konjunkturellen Stabilisatorfunktion des Handwerks auszugehen, wenn sich die zyklischen Ausschläge des Handwerksumsatzes gegenläufig zu den zyklischen Ausschlägen der Gesamtwirtschaft verhalten (antizyklisch) oder diese gegebenenfalls nur geringfügig nachvollziehen. $^{37}$

Ein Vergleich der für das Handwerk und die Gesamtwirtschaft näherungsweise bestimmten Konjunkturschwankungen im Zeitraum 1994-2009 wirft dann auch ein neues Licht auf die Ausgangsfrage (vgl. Abbildung 4). ${ }^{38}$ Gemessen an den prozentualen Abweichungen vom längerfristigen Trendverlauf erlebte die Handwerkskonjunktur demnach im Jahr 2009 zwar einen deutlichen Rückgang gegenüber dem unter konjunkturellen Gesichtspunkten noch besonders positiv verlaufenen Vorjahr, dennoch war kein Einbruch wie in der Gesamtwirtschaft $\mathrm{zu}$ verzeichnen. Relativ gesehen hat das Handwerk demzufolge in der Wirtschaftskrise 2008/2009 durchaus bis zu einem gewissen Grad eine stabilisierende Wirkung entfaltet. Aufgrund der deutlichen Abweichung zwischen den zyklischen Ausschlägen des Handwerksumsatzes und des Bruttoinlandsprodukts dürfte diese Aussage selbst unter der Annahme gelten, dass das ermittelte Schätzergebnis aufgrund von Ungenauigkeiten am aktuellen Rand der Zeitreihen ein Stück weit verzerrt ist. Bekräftigt wird diese Schlussfolgerung wiederum dadurch, dass bspw. die im Jahresverlauf 2009 laut den Konjunkturumfragen vergleichsweise stabil gebliebene Geschäftslage im Handwerk (vgl. Abbildung 3, S. 127) in deutlich plau-

36 Vgl. Lageman, B. u. a. (2005), S. 4ff. Vgl. hierzu auch Abschnitt 6.2.

37 Vgl. Dürig, W. u. a. (2004), S. 82.

38 Das zyklische Verlaufsmuster wurde folgendermaßen berechnet: Mit Hilfe des Hodrick-Prescott-Filters für Jahresdaten $(\lambda=100)$ wurde der sog. HP-Trend für die logarithmierten Ausgangsreihen geschätzt und von diesen dann jeweils abgezogen. Im Ergebnis stehen die zyklischen Komponenten der betrachteten Zeitreihen, die sich (näherungsweise) als prozentuale Abweichungen der Ausgangsreihen von ihrem jeweiligen Trendverlauf ergeben. Da der HP-Trend am aktuellen Rand einer Zeitreihe Ungenauigkeiten aufweisen kann („Endpunktproblem“), wurden in der Schätzung zudem die beim Abschluss der vorliegenden Untersuchung aktuellen Prognosewerte für das Jahr 2010 berücksichtigt. Hierbei wurde für das Jahr 2010 ein Rückgang des nominalen Handwerksumsatzes um -1 \% und ein Anstieg des nominalen Bruttoinlandsprodukts um 2,3 \% unterstellt. Vgl. hierzu das interne Protokoll zur Sitzung der ZDH-Planungsgruppen „Konjunkturprognosen und Handwerksstatistik“ am 20. April 2010 in Berlin und ifo (2010). Zur näheren Beschreibung des methodischen Vorgehens siehe auch Abschnitt 5.1. 
siblerer Übereinstimmung zum ermittelten zyklischen Verlaufsmuster steht als die nichttrendbereinigten Ergebnisse zur Entwicklung der Handwerksumsätze im Jahr 2009.

Abbildung 4: Prozentuale Trendabweichungen im Handwerk und in der Gesamtwirtschaft (zyklische Komponenten; nominale Jahresdaten; 1994 bis 2009)

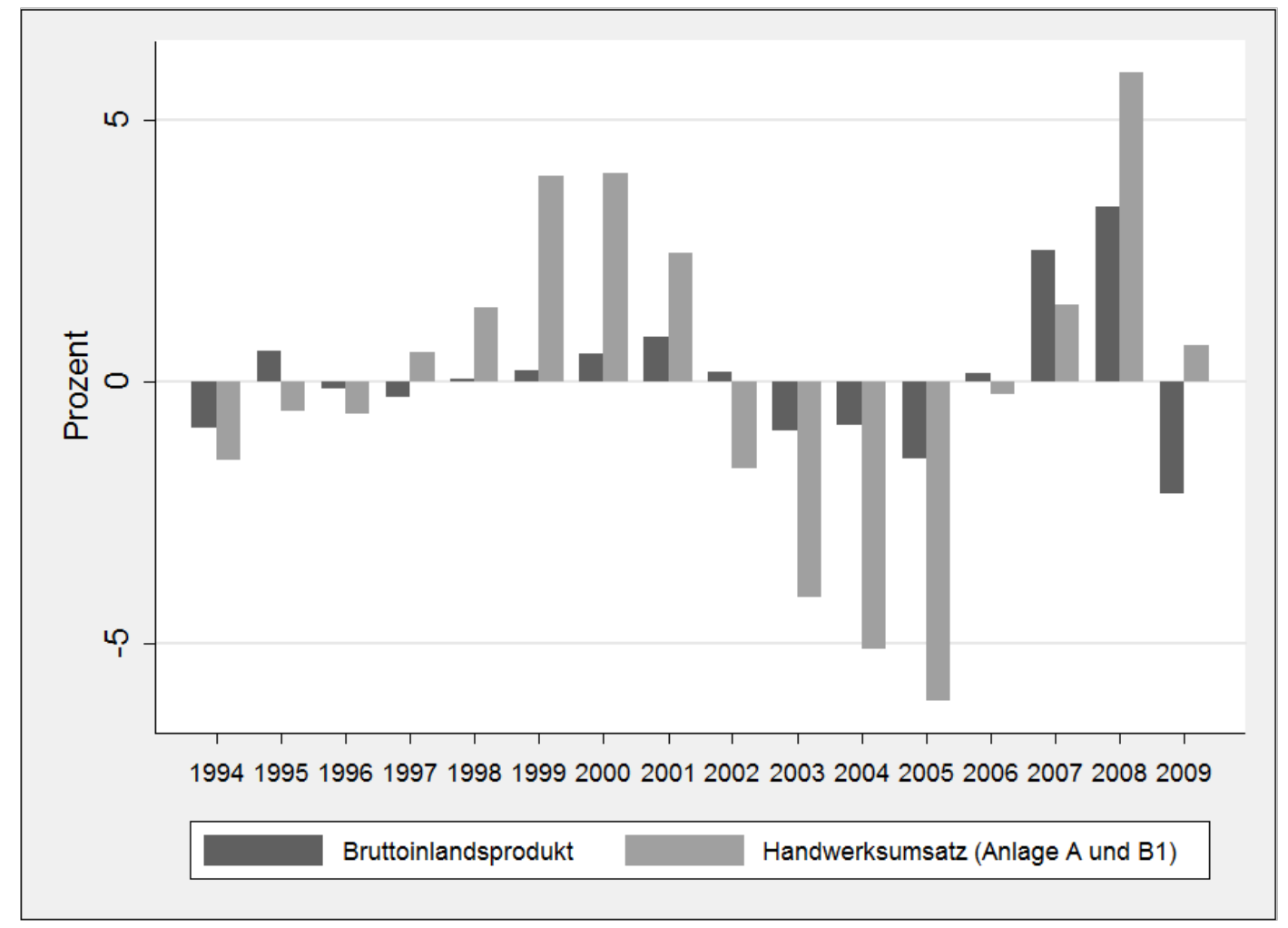

Quelle: Statistisches Bundesamt, Zentralverband des Deutschen Handwerks, eigene Berechnungen

Vor diesem Hintergrund wird im Folgenden der Frage nachgegangen, wodurch sich die konjunkturelle Lage des Handwerks während der Wirtschaftskrise 2008/2009 erklärt. Zugleich soll hierbei untersucht werden, inwieweit die Handwerkswirtschaft eine allgemeine Stabilisatorfunktion besitzt. Eine uneingeschränkte Gültigkeit dieses Phänomens scheint bereits angesichts der Tatsache fraglich, dass das Handwerk z.B. in der gesamtwirtschaftlichen Schwächeperiode der Jahre 2002 bis 2005 - gemessen an der Stimmungslage und am ermittelten zyklischen Verlaufsmuster - offenbar keineswegs stabilisiert hat (vgl. Abbildung 3 und Abbildung 4). Es ist daher nach spezifischen Ausgestaltungen der konjunkturellen Gesamtkonstellation zu fragen, die eine Stabilisatorfunktion des Handwerks eher begünstigen als andere. Hierzu werden im Rahmen von Kapitel 6 die gesamtwirtschaftlichen Determinanten der Handwerkskonjunktur verdeutlicht, welche angesichts der sektoralen Struktur des Handwerks einen wichtigen Beitrag zur Erklärung einer etwaigen Stabilisatorfunktion liefern. Die Grundlage zum Verständnis der Auswirkungen dieser gesamtwirtschaftlichen Verflechtungen auf die konjunkturellen Ausschläge der Handwerkswirtschaft liefert jedoch zunächst eine Verdeutlichung von statistisch messbaren Konjunkturmustern im Handwerk (vgl. Kapitel 5). 


\section{Datenbasis}

Die Analyse in Kapitel 5 und 6 basiert auf den vierteljährlichen Beschäftigungs- und Umsatzdaten der Handwerksberichterstattung. Das primäre Ziel dieser vom Statistischen Bundesamt seit 1960 veröffentlichten Handwerksstatistik ist eine Abbildung des konjunkturellen Verlaufs im Handwerk. Bis zum Jahr 2008 wurden hierzu die Daten im Rahmen einer vierteljährlichen Befragung von etwa 50.000 Handwerksbetrieben erhoben, wobei die Ergebnisse der verschiedenen Handwerkszählungen jeweils zur strukturellen Überprüfung und Ergänzung der erhobenen Daten herangezogen wurden. Seit 2008 wird die Primärerhebung durch eine Auswertung von unterjährigen Verwaltungsdaten ersetzt. Hierbei werden zum einen die Angaben zu den sozialversicherungspflichtigen und geringfügig Beschäftigten aus den Meldungen zur Sozialversicherung für die Bundesagentur für Arbeit genutzt. Zum anderen fließen die Umsatzsteuervoranmeldungen der Unternehmen für die Finanzverwaltung in die statistische Erhebung mit ein. ${ }^{39}$

Seit der letzten Handwerkszählung 1995 werden vom Statistischen Bundesamt in der Handwerksberichterstattung keine Absolutzahlen über Beschäftigte und Umsatz im Handwerk mehr veröffentlicht. Stattdessen erfolgt nur eine Darstellung von Indexwerten bzw. prozentualen Veränderungen. Die im Rahmen der vorliegenden Untersuchung ausgewerteten Zeitreihendaten wurden daher vom Zentralverband des Deutschen Handwerks (ZDH) bezogen. Hierbei handelt es sich um vierteljährliche Daten über Beschäftigte und Umsatz im zulassungspflichtigen Handwerk (Anlage A HwO) im Zeitraum 1996 bis 2009, die vom ZDH auf Grundlage der Handwerksberichterstattung und der Handwerkszählung 1995 näherungsweise wieder auf ihre Absolutwerte rückgerechnet wurden. ${ }^{40}$ Neben dieser Aufbereitungsform besitzen die ZDH-Daten den weiteren Vorteil, dass sie eine separate Betrachtung von handwerklichen Gewerbegruppen erlauben. Angesichts der großen Heterogenität der Handwerkswirtschaft ist aus konjunkturanalytischer Sicht eine Zusammenfassung der handwerklichen Gewerbezweige in möglichst homogene Gewerbegruppen geboten. Gleichwohl ist zu beachten, dass innerhalb der Gewerbegruppen auch weiterhin erhebliche konjunkturelle und strukturelle Unterschiede bestehen können. ${ }^{41}$

Tabelle 3 führt die sieben Gewerbegruppen des Handwerks auf. Beispielhaft werden den einzelnen Gruppen dabei solche Gewerbe zugeordnet, die nach Anlage A der Handwerksordnung einer Zulassungspflicht unterliegen. Die Aufzählung macht bereits deutlich, dass durch die nötige Beschränkung auf die zulassungspflichtigen A-Handwerke eine Reihe von handwerklichen Berufen in der folgenden Analyse außer Acht gelassen werden muss. Dies führt

\footnotetext{
39 Vgl. Müller, K. (2010), S. 13ff.
}

40 Erst seit dem Jahr 2008 wird das zulassungsfreie Handwerk (Anlage B Abschnitt 1 HwO) wieder in die Handwerksberichterstattung einbezogen. Für die Längsschnittbetrachtung der vorliegenden Studie konnten die B1-Handwerke daher nicht berücksichtigt werden. Vgl. Neuhäuser, J. (2008), S. 404f. 
freilich dazu, dass die Repräsentativität der Untersuchungsergebnisse ein Stück weit eingeschränkt ist. Angesichts der Tatsache, dass nach Schätzungen des ZDH auf die A-Handwerke jedoch in etwa $90 \%$ des Umsatzes und ca. $75 \%$ der Beschäftigten im Gesamthandwerk entfallen (Stand 2008), kann hinsichtlich der folgenden Einschätzungen zur Handwerkskonjunktur dennoch eine weitreichende Aussagekraft unterstellt werden.

Tabelle 3: Einteilung des zulassungspflichtigen Handwerks (Anlage A HwO) in Gewerbegruppen mit Beispielen

\section{Bauhauptgewerbe \\ - Maurer und Betonbauer \\ - Zimmerer \\ - Dachdecker \\ - Straßenbauer \\ - Gerüstbauer}

\section{Ausbaugewerbe}

- Maler und Lackierer

- Klempner

- Installateur und Heizungsbauer

- Elektrotechniker

- Tischler

- Glaser

- Ofen- und Luftheizungsbauer

\section{Handwerke für den gewerblichen Bedarf}

- Feinwerkmechaniker

- Elektromaschinenbauer

- Landmaschinenmechaniker

- Kälteanlagenbauer

- Metallbauer

- Informationstechniker

\section{Kraftfahrzeuggewerbe}

- Karosserie- und Fahrzeugbauer

- Zweiradmechaniker

- Kraftfahrzeugtechniker

\section{Lebensmittelgewerbe}

- Bäcker

- Konditoren

- Fleischer

\section{Gesundheitsgewerbe}

- Augenoptiker

- Zahntechniker

- Hörgeräteakustiker

- Orthopädieschuhmacher

- Orthopädietechniker

\section{Personenbezogene}

Dienstleistungsgewerbe

- Friseure

- Schornsteinfeger

- Steinmetzen und Steinbildhauer

- Boots- und Schiffbauer

Quelle: Neuhäuser (2008), S. 406 und ZDH (2009b), S. 48.

\section{Allgemeine Konjunkturmuster im Handwerk}

\subsection{Methodisches Vorgehen}

Als methodische Grundlage zur möglichst weitreichenden Bereinigung von nicht-konjunkturellen Einflüssen dient das Komponentenmodell der Zeitreihenanalyse. Es umfasst im Kern 
vier Bausteine: Der Trend bestimmt die längerfristige Entwicklungsrichtung einer Zeitreihe. Dagegen misst die Konjunkturkomponente zyklisch wiederkehrende Schwankungen und wird daher auch als zyklische Komponente bezeichnet. Die Saisonkomponente bildet jahreszeitliche Schwankungen ab, wohingegen die irreguläre Komponente alle restlichen (unsystematischen) Einflüsse erfasst. ${ }^{42}$ Es würde nun für das Vorliegen einer Stabilisatorfunktion des Handwerks sprechen, wenn sich die zyklischen Komponenten der handwerklichen Umsatz- und Beschäftigungsentwicklung gegenläufig (antizyklisch) zur gesamtwirtschaftlichen Konjunktur verhalten oder gegebenenfalls die dortigen zyklischen Ausschläge nur geringfügig nachvollziehen. $^{43}$

Die statistische Filterung einer Zeitreihe bietet eine Möglichkeit, nicht-konjunkturelle Einflüsse möglichst auszuschließen und somit die zyklische Komponente zu ermitteln. Im Rahmen dieser Untersuchung wurden zu diesem Zweck verschiedene methodische Schritte durchgeführt. Zunächst wurden die Zeitreihendaten mit Hilfe des „Berliner Verfahrens“44 einer Saisonbereinigung unterzogen. Eine saisonbereinigte Reihe ist jedoch noch immer durch ihren längerfristigen Trend überlagert. Zur Messung von konjunkturell bedingten Schwankungen musste daher zusätzlich eine entsprechende Trendbereinigung erfolgen. Die Zeitreihendaten wurden hierzu zunächst logarithmiert. Für diese Reihen wurde daraufhin mit Hilfe des Hodrick-Prescott-Filters für Quartalsdaten $(\lambda=1600)$ der sog. HP-Trend geschätzt, welcher zum Abschluss von der logarithmierten Ausgangsreihe abgezogen wurde. Im Ergebnis steht die zyklische Komponente eine Zeitreihe, welche näherungsweise die prozentuale Abweichung der saisonbereinigten Ausgangsreihe von ihrem Trend darstellt. ${ }^{45}$

In der modernen Konjunkturforschung hat insbesondere eine Charakterisierung von zyklischen Schwankungen durch sogenannte Konjunkturmuster („stilisierte Fakten“) weite Verbreitung gefunden. ${ }^{46}$ Bei stilisierten Fakten handelt es sich um „feste Muster im zeitlichen Nebeneinander vieler Zeitreihen“ ${ }^{\star 47}$. Entsprechend werden im folgenden Abschnitt für den Zeitraum 1996 bis 2009 die zentralen stilisierten Fakten der Handwerkskonjunktur anhand des Korrelationskoeffizienten $\rho$ und der Standardabweichung $s$ beschrieben. Der Korrelationskoeffizient misst die Stärke des linearen Zusammenhangs zwischen zwei Zeitreihen. ${ }^{48}$ Zwei

42 Vgl. Maußner, A. (1994), S. 6 f. Wird eine Zeitreihe als Summe (Produkt) der vier Komponenten verstanden, wird von einem additiven (multiplikativen) Komponentenmodell gesprochen.

43 Vgl. Dürig, W. u. a. (2004), S. 82.

44 Das Berliner Verfahren (BV) ist ein mathematisch-statistisches Verfahren, das vom Statistischen Bundesamt in seiner aktuellen Version BV4.1 zur Zeitreihenzerlegung benutzt wird. Zur ausführlichen Information vgl. Speth, H.-T. (2004).

45 Zur ausführlichen methodischen Beschreibung vgl. Maußner, A. (1994), S. 7ff und S. 248ff; Winker, P. (2007), S. 215ff.

46 Vgl. Tichy, G. (1994), S. 39.

47 Maußner, A. (1994), S. 14.

48 Der Korrelationskoeffizient ist auf $-1 \leq \rho \leq 1$ normiert. Bei $\rho=0$ besteht kein Zusammenhang. Werte von $+1(-1)$ lassen auf einen vollständig positiven (negativen) Zusammenhang schließen. 
Reihen verhalten sich prozyklisch (antizyklisch) zueinander, wenn ein signifikant positiver (negativer) Korrelationskoeffizient zum Zeitpunkt $t$ vorliegt. Die zyklische Komponente einer Zeitreihe besitzt dabei im Vergleich zu einer Referenzreihe einen Vorlauf (Nachlauf), wenn sie im Vergleich zum Höhepunkt der Referenzreihe ihr Maximum $n$ Perioden vorher (nachher) erreicht. Der Korrelationskoeffizient ist in diesem Fall bei $i=n$ am größten (vgl. Abbildung 5). Die Standardabweichung misst hingegen die Schwankungsintensität (Volatilität) der zyklischen Komponente einer Zeitreihe. ${ }^{49}$

Abbildung 5: Idealtypische Verlaufseigenschaften von Konjunkturzeitreihen

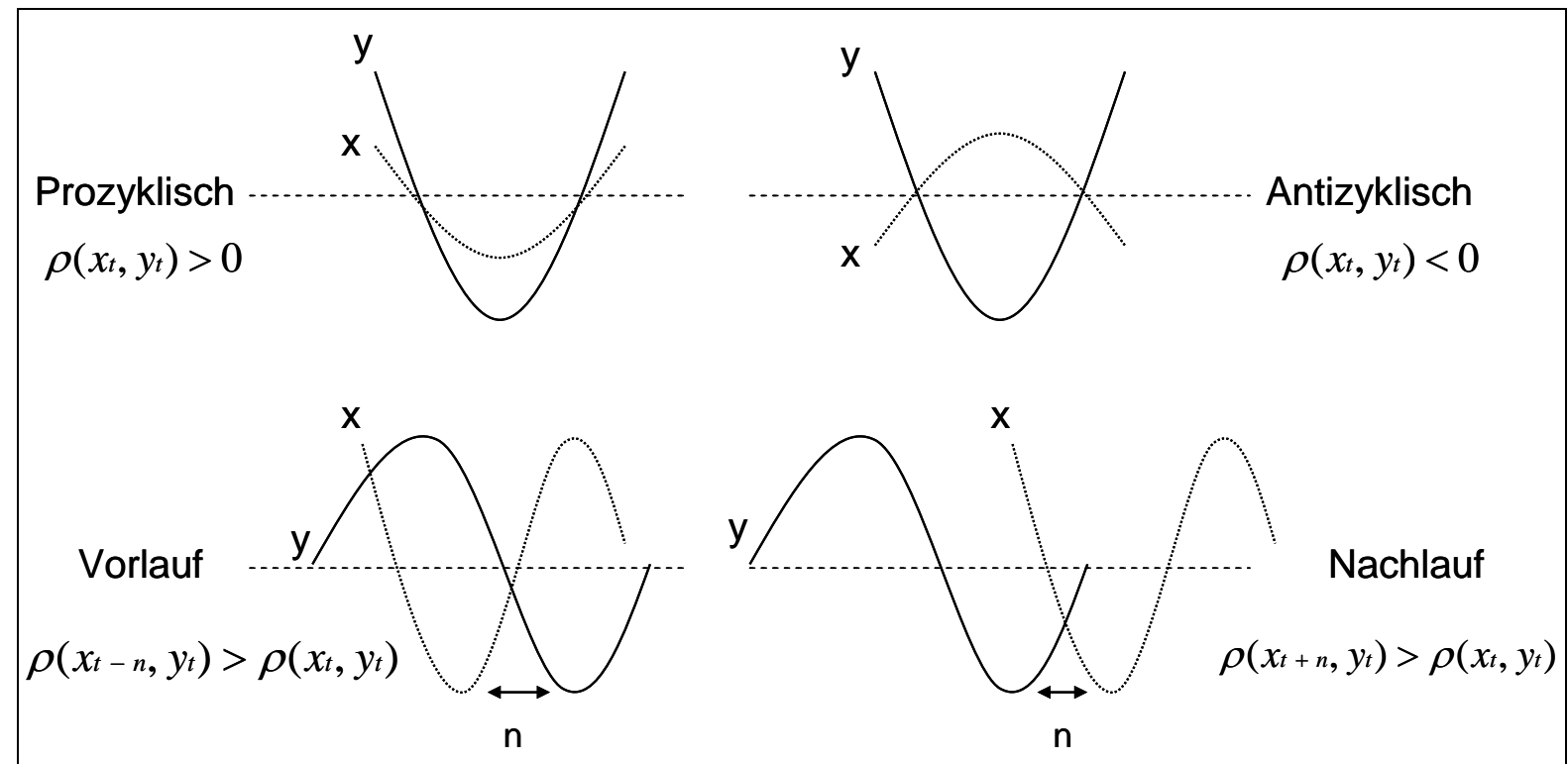

Quelle: Eigene Darstellung auf Grundlage von Maußner (1994), S. 15

\subsection{Stilisierte Fakten zur Handwerkskonjunktur}

Tabelle 4 liefert einen Eindruck zum Zusammenhang zwischen der nominalen Umsatzentwicklung in den Gewerbegruppen des Handwerks und dem Bruttoinlandsprodukt als Maß für den gesamtwirtschaftlichen Konjunkturzyklus (Referenzreihe). ${ }^{50}$ Neben den Standardweichungen der jeweiligen zyklischen Komponente sind in den Spalten die Korrelationskoeffizienten der Reihen zur zyklischen BIP-Komponente abgebildet. Anhand $\mathrm{t} \pm \mathrm{i}(\mathrm{i}=0,1,2,3,4)$

49 Vgl. Maußner, A. (1994), S. 15 f.

50 Der Vergleich zwischen Handwerksumsätzen und dem BIP ist mit zwei methodischen Problemen verbunden. Erstens ist die grundsätzliche Vergleichbarkeit aufgrund einer unterschiedlichen Berücksichtigung der Vorleistungen nicht unproblematisch. In der Entstehungsrechnung der Volkswirtschaftlichen Gesamtrechnung wird das BIP u.a. durch einen Abzug der Vorleistungen vom Bruttoproduktionswert errechnet, welcher somit besser vergleichbar zu den nicht von Vorleistungen bereinigten Handwerksumsätzen wäre. Angesichts des in etwa gleichgerichteten Verlaufs von BIP und Bruttoproduktionswert ist der vorgenommene Vergleich jedoch dennoch möglich. Zweitens liegt kein geeigneter Preisindex für Produkte und Leistungen des Handwerks vor, weshalb in der vorliegenden Untersuchung auf die Verwendung realer Größen verzichtet werden musste. Vgl. Müller, K. u. a. (2009), S. 109, Fußnote 128 zum Problem der Preisbereinigung von Handwerksumsätzen. 
ist ersichtlich, um wie viele Quartale hinsichtlich der Referenzreihe ein Vorlauf (i < 0) bzw. Nachlauf (i $>0)$ besteht.

Tabelle 4: Zeitliche Korrelationen zwischen Umsatzschwankungen im zulassungspflichtigen Handwerk und der gesamtwirtschaftlichen Konjunkturlage

\begin{tabular}{|c|c|c|c|c|c|c|c|c|c|c|}
\hline \multirow{2}{*}{$\begin{array}{l}\text { Gewerbe- } \\
\text { gruppen des } \\
\text { Handwerks }\end{array}$} & \multirow{2}{*}{$S_{x}^{b)}$} & \multicolumn{4}{|c|}{ Vorlauf ${ }^{c) d)}$} & \multirow[b]{2}{*}{$\mathrm{t}^{c) d)}$} & \multicolumn{4}{|c|}{ Nachlauf $^{c) d)}$} \\
\hline & & $t-4$ & $\mathrm{t}-3$ & $\mathrm{t}-2$ & $\mathrm{t}-1$ & & $\mathrm{t}+1$ & $t+2$ & $t+3$ & $\mathrm{t}+4$ \\
\hline $\mathrm{Bau}^{a)}$ & 3,9 & 0,05 & 0,13 & 0,28 & $0,41^{*}$ & $0,52 *$ & $0,41^{*}$ & 0,20 & 0,04 & $-0,06$ \\
\hline Ausbau $^{a)}$ & 3,6 & 0,06 & 0,15 & 0,33 & $0,43^{*}$ & $0,52 *$ & $0,43^{*}$ & 0,29 & 0,22 & 0,16 \\
\hline $\begin{array}{l}\text { Gewerblicher } \\
\text { Bedarf }^{a)}\end{array}$ & 4,6 & 0,03 & 0,25 & $0,53^{*}$ & $0,85^{*}$ & $0,89 *$ & $0,70^{*}$ & $0,40 *$ & 0,17 & 0,04 \\
\hline $\begin{array}{l}\text { Kraftfahr- } \\
\text { zeuge }^{a)}\end{array}$ & 2,6 & $-0,23$ & $-0,33$ & $-0,40 *$ & $-0,40^{*}$ & $-0,23$ & 0,02 & 0,10 & 0,05 & $-0,01$ \\
\hline $\begin{array}{l}\text { Lebens- } \\
\text { mittel }^{a)}\end{array}$ & 2,0 & 0,26 & $0,37 *$ & $0,46^{*}$ & $0,44^{*}$ & $0,39 *$ & 0,28 & 0,18 & 0,10 & 0,07 \\
\hline Gesundheit $^{a}$ ) & 4,2 & 0,26 & 0,17 & 0,10 & $-0,01$ & $-0,02$ & 0,02 & $-0,04$ & $-0,13$ & $-0,25$ \\
\hline $\begin{array}{l}\text { Personen- } \\
\text { bezogene } \\
\text { Dienstleis- } \\
\text { tungen }^{a} \text { ) }\end{array}$ & 2,6 & 0,32 & 0,31 & 0,23 & 0,08 & 0,00 & $-0,06$ & $-0,11$ & $-0,22$ & $-0,23$ \\
\hline
\end{tabular}

a) Zyklische Komponenten der Umsatzentwicklung: HP-gefilterte natürliche Logarithmen $(\mu=1600)$ von saisonbereinigten Quartalsdaten (BV 4.1)

b) Standardabweichung zur Messung der Volatilität

c) Referenzreihe: Zyklische Komponente des nominalen Bruttoinlandprodukts

d) Korrelationskoeffizienten der Reihe zum angeführten Zeitpunkt mit der zyklischen Komponente des Bruttoinlandsprodukts zum Zeitpunkt t. Werte von $\rho=|0,4|$ und geringer deuten auf einen schwachen Zusammenhang. Werte zwischen $\rho=|0.41|$ und $\rho=|0.60|$ weisen auf eine moderaten Zusammenhang. Werte von $\rho=|0.61|$ und höher bedeuten einen starken Zusammenhang.

e) Signifikanzniveau: * $1 \%$

f) Quelle: Zentralverband des Deutschen Handwerks, eigene Berechnungen

Mit Blick auf die Konjunkturabhängigkeit der einzelnen Gewerbegruppen fällt zunächst eine grundsätzliche Zweiteilung auf. Zunächst sind die Bau- und Ausbauhandwerke sowie die Handwerke für den gewerblichen Bedarf zu nennen. Diese Gewerbegruppen zeichnen sich durch einen prozyklischen Gleichlauf aus, wobei der Zusammenhang zum Bruttoinlandsprodukt bei den Handwerken für den gewerblichen Bedarf am größten ausfällt. Auf Grundlage des errechneten Korrelationskoeffizienten kann in diesem Fall auf einen sehr engen Zusam- 
menhang zur Gesamtwirtschaft geschlossen werden. Bei den Bau- und Ausbauhandwerken besteht hingegen nur ein moderater Zusammenhang zur Gesamtkonjunktur. Im Falle der Lebensmittelhandwerke ist ebenfalls ein tendenziell prozyklisches Verhalten zu beobachten, wobei jedoch ein Vorlauf von ein bis zwei Quartalen besteht und ebenfalls kein starker Zusammenhang feststellbar ist.

Des Weiteren sind Gewerbegruppen zu nennen, die sich durch keine bzw. eine sehr geringe Konjunkturabhängigkeit auszeichnen. Die Kfz-Handwerke haben sich im Beobachtungszeitraum antizyklisch verhalten, wobei die Umsatzentwicklung dem Bruttoinlandsprodukt um ein bis zwei Quartale voran gelaufen ist. Der Zusammenhang ist hier jedoch nur schwach ausgeprägt. Die handwerklichen Gewerbegruppen „Gesundheit“ und „Personenbezogene Dienstleistungen“ sind von der gesamtwirtschaftlichen Wirtschaftslage offenbar kaum abhängig, da kein merklicher Zusammenhang zum gesamtwirtschaftlichen Konjunkturzyklus besteht.

Die zyklische Komponente mit der größten Volatilität weisen die Handwerke für den gewerblichen Bedarf auf. Gemessen an der Standardabweichung sind demnach die konjunkturellen Ausschläge in diesem Handwerksbereich als besonders hoch anzusehen. Des Weiteren besitzt die Umsatzentwicklung im Bau- und Ausbauhandwerk für den Untersuchungszeitraum eine relativ hohe Volatilität. In Kapitel 6 wird auf die Hintergründe dieser Befunde detailliert eingegangen. Zunächst überraschend ist jedoch die hohe Schwankungsintensität der Umsatzentwicklung in den Gesundheitshandwerken. Hierbei ist jedoch zu beachten, dass sich gesundheitspolitische Vorgaben zur Kostenübernahme von Hilfsmitteln (Brillen, Hörgeräte, Prothesen etc.) durch die Krankenkassen unmittelbar auf die Wirtschaftslage im Gesundheitshandwerk auswirken. ${ }^{51}$ Die hohe Volatilität in diesem Fall dürfte sich daher durch die zurückliegenden Kostenstrukturreformen im Gesundheitswesen erklären, wovon die Gesundheitshandwerke in Form von deutlichen Umsatz- und Beschäftigungsschwankungen betroffen waren. ${ }^{52}$

Analog zum soeben vorgenommenen Vergleich stellt Tabelle 5 die zyklischen Ausschläge der Handwerksbeschäftigung dem konjunkturellen Verlauf der gesamtwirtschaftlichen Erwerbstätigkeit $^{53}$ gegenüber. Bei den Handwerken für den gewerblichen Bedarf zeigt sich auch in diesem Fall ein prozyklisches Verhalten mit starkem Gleichlauf zur Gesamtwirtschaft. Im Falle der Handwerke für personenbezogene Dienstleistungen ist ebenfalls ein prozyklisches Verhalten mit der Tendenz zum Vorlauf um ein Quartal zu beobachten, wobei jedoch kein starker Zusammenhang vorliegt. Von besonderem Interesse ist indes die Beschäftigungsentwicklung in den Bau-, Ausbau-, Kfz-, und Lebensmittelhandwerken. Hier zeigt sich je nach

51 Vgl. Dürig u.a. 2004, S. 253.

52 Vgl. Dispan, J. (2003), S. 119. Siehe hierzu beispielhaft auch die folgenden ZDH-Konjunkturberichte: Ausgabe 1/1999, S. 8; Ausgabe 2/1999, S. 12; Ausgabe 2/2004, S. 12, 14 und 16.

53 „Zu den Erwerbstätigen in den Volkswirtschaftlichen Gesamtrechnungen zählen alle Personen, die als Arbeitnehmer (Arbeiter, Angestellte, Beamte, geringfügig Beschäftigte, Soldaten) oder als Selbstständige beziehungsweise als mithelfende Familienangehörige eine auf wirtschaftlichen Erwerb gerichtete Tätigkeit ausüben.“ Siehe die Erwerbstätigendefinition auf http://www.destatis.de. 
Gewerbegruppe ein Nachlauf der Beschäftigung um zwei bis vier Quartale, wobei in allen Fällen ein starker Zusammenhang zum Verlauf der gesamtwirtschaftlichen Erwerbstätigkeit vorliegt. Für diesen Handwerksbereich kann somit im Vergleich zur Gesamtwirtschaft auf ein beharrendes Beschäftigungsverhalten geschlossen werden und folglich die oben diskutierte These von Dürig u. a. (2004) nicht bestätigt werden.

Eine relativ gesehen hohe Volatilität der Beschäftigung zeigt sich daneben bei den Bauhandwerken und den Handwerken für den gewerblichen Bedarf. Die Gesundheitshandwerke fallen zudem auch im Falle der Beschäftigungsentwicklung durch ein spezifisches Konjunkturmuster auf (antizyklisch, Nachlauf, hohe Volatilität), was auf die diskutierten Umbrüche in dieser Gewerbegruppe zurückzuführen sein dürfte.

Tabelle 5: Zeitliche Korrelationen zwischen Beschäftigungsschwankungen im zulassungspflichtigen Handwerk und gesamtwirtschaftlicher Erwerbstätigkeit

\begin{tabular}{|c|c|c|c|c|c|c|c|c|c|c|}
\hline \multirow{2}{*}{$\begin{array}{l}\text { Gewerbe- } \\
\text { gruppen des } \\
\text { Handwerks }\end{array}$} & \multirow{2}{*}{$S_{X}{ }^{b)}$} & \multicolumn{4}{|c|}{ Vorlauf $^{c) d \text { ) }}$} & \multirow[b]{2}{*}{$\mathrm{t}^{c) d)}$} & \multicolumn{4}{|c|}{ Nachlauf $^{c) d)}$} \\
\hline & & $\mathrm{t}-4$ & $t-3$ & $t-2$ & $\mathrm{t}-1$ & & $t+1$ & $t+2$ & $t+3$ & $t+4$ \\
\hline $\mathrm{Bau}^{a)}$ & 2,1 & 0,09 & 0,26 & $0,44^{*}$ & $0,60^{*}$ & $0,69 *$ & $0,8^{*}$ & $0,87^{*}$ & $0,89 *$ & $0,84^{*}$ \\
\hline Ausbau $^{a}$ ) & 1,4 & $0,40 *$ & $0,50^{*}$ & $0,58^{*}$ & $0,62 *$ & $0,59 *$ & $0,65^{*}$ & $0,67 *$ & $0,65 *$ & $0,60 *$ \\
\hline $\begin{array}{l}\text { Gewerblicher } \\
\text { Bedarf }^{a)}\end{array}$ & 1,7 & 0,34 & $0,52^{*}$ & $0,67 *$ & $0,78^{*}$ & $0,82 *$ & $0,79 *$ & $0,68^{*}$ & $0,54^{*}$ & $0,41^{*}$ \\
\hline $\begin{array}{l}\text { Kraftfahr- } \\
\left.\text { zeuge }^{a}\right)\end{array}$ & 0,9 & $-0,14$ & $-0,04$ & 0,10 & 0,27 & $0,44^{*}$ & $0,57^{*}$ & $0,62^{*}$ & $0,59 *$ & $0,50 *$ \\
\hline $\begin{array}{l}\text { Lebens- } \\
\text { mittel }^{a)}\end{array}$ & 1,1 & $-0,06$ & 0,10 & 0,25 & $0,37 *$ & $0,44^{*}$ & $0,56^{*}$ & $0,64 *$ & $0,69 *$ & $0,72 *$ \\
\hline Gesundheit $^{a}$ ) & 2,6 & 0,21 & 0,04 & $-0,13$ & $-0,28$ & $-0,40 *$ & $-0,47 *$ & $-0,51 *$ & $-0,54 *$ & $-0,54^{*}$ \\
\hline $\begin{array}{l}\text { Personenbe- } \\
\text { zogene } \\
\text { Dienstleis- } \\
\text { tungen }^{a)}\end{array}$ & 0,6 & 0,27 & $0,44^{*}$ & $0,55^{*}$ & $0,60 *$ & $0,57^{*}$ & $0,55^{*}$ & $0,50^{*}$ & $0,45^{*}$ & $0,43^{*}$ \\
\hline
\end{tabular}

a) Zyklische Komponenten der Beschäftigungsentwicklung: HP-gefilterte natürliche Logarithmen $(\mu=1600)$ von saisonbereinigten Quartalsdaten (BV 4.1)

b) Standardabweichung zur Messung der Volatilität

c) Referenzreihe: Zyklische Komponente der gesamtwirtschaftlichen Erwerbstätigkeit

d) Korrelationskoeffizienten der Reihe zum angeführten Zeitpunkt mit der zyklischen Komponente der Erwerbstätigkeit zum Zeitpunkt t. Werte von $\rho=|0,4|$ und geringer deuten auf einen schwachen $\mathrm{Zu}$ sammenhang. Werte zwischen $\rho=|0.41|$ und $\rho=|0.60|$ weisen auf eine moderaten Zusammenhang. Werte von $\rho=|0.61|$ und höher bedeuten einen starken Zusammenhang.

e) Signifikanzniveau: * $1 \%$

f) Quelle: Zentralverband des Deutschen Handwerks, Bundesbank, eigene Berechnungen 


\subsection{Zwischenfazit}

Der vorgenommene Vergleich der Umsatz- und Beschäftigungsentwicklung im Handwerk mit den entsprechenden gesamtwirtschaftlichen Größen lässt verschiedene Schlüsse zu. Gemessen an der Umsatzentwicklung bleibt festzuhalten, dass sich wichtige Bereiche der Handwerkskonjunktur (Bau, Ausbau, Gewerblicher Bedarf, abgeschwächt auch Lebensmittel) insgesamt prozyklisch zum Auf und Ab der Gesamtkonjunktur verhalten. Gemessen am Umsatz machen diese Gewerbegruppen knapp 70 \% des zulassungspflichtigen Handwerks aus (vgl. Abbildung $6)$.

Abbildung 6: Prozentuale Verteilung des Umsatzes im zulassungspflichtigen Handwerk (Stand 2008)

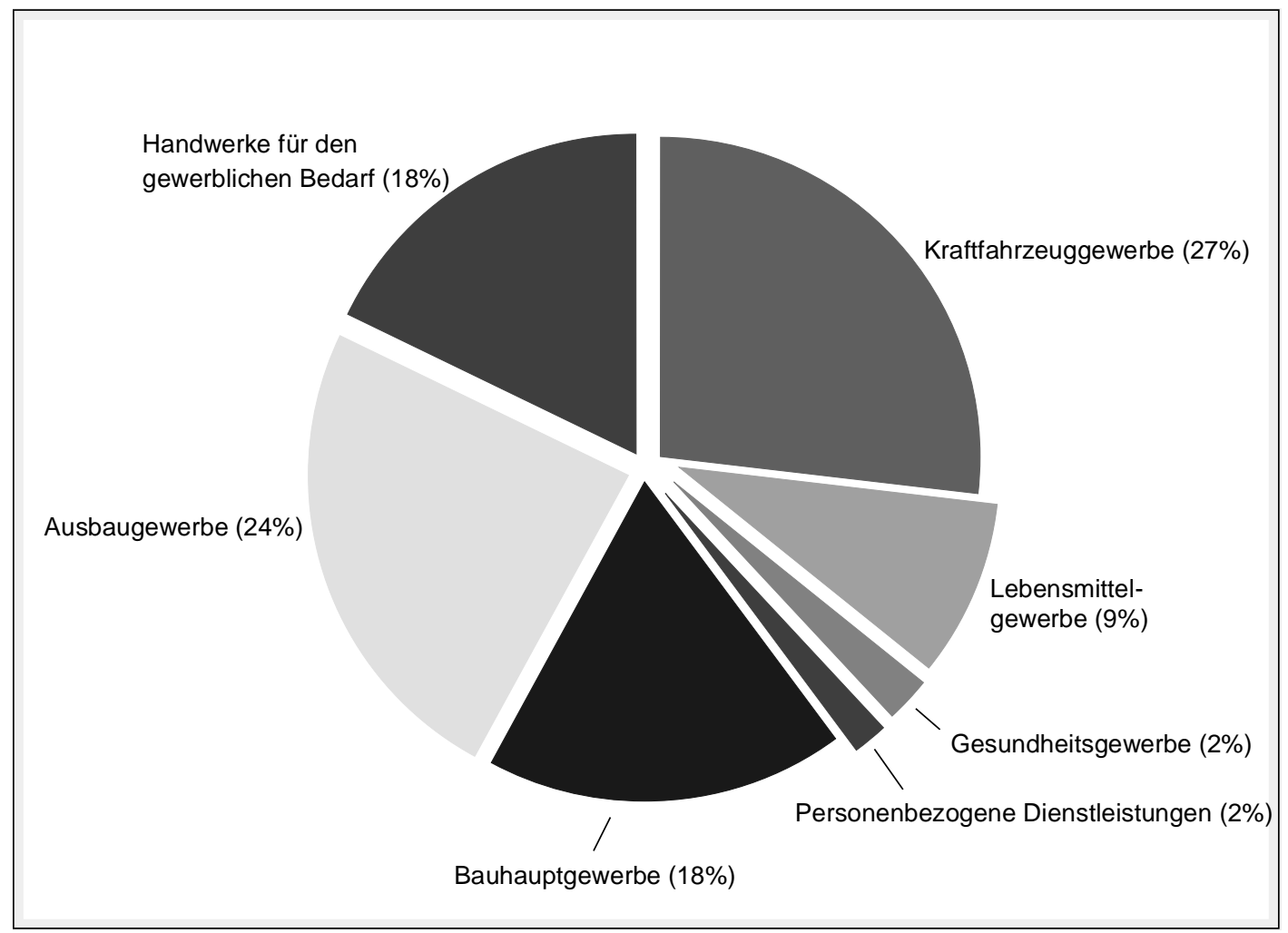

Quelle: Zentralverband des Deutschen Handwerks

Nach den oben definierten Kriterien spricht dieser Befund eher gegen die Gültigkeit der Stabilisatorhypothese. Allerdings muss hierbei beachtet werden, dass nur bei den Handwerken für den gewerblichen Bedarf von einem starken Zusammenhang gesprochen werden kann. Zudem sind am Umsatz gemessen ca. 30 \% des zulassungspflichtigen Handwerks kaum konjunkturabhängig und dürften daher weit stärker durch branchenspezifische Entwicklungen geprägt sein. Das Kfz-Gewerbe hat sich z.B. im Untersuchungszeitraum mit einem leichten Vorlauf antizyklisch entwickelt. Die Gesundheitshandwerke und die Handwerke für personenbezogene Dienstleistungen weisen zudem keinen nennenswerten statistischen Zusammenhang zur Gesamtwirtschaft auf, was wie im Falle des Kfz-Gewerbes eher für die Gültigkeit der Stabilisatorhypothese spricht. Mit Blick auf die Umsatzentwicklung des zulassungspflichtigen Handwerks kann daher festgehalten werden, dass sich die Handwerkswirtschaft in 
einen eher konjunkturabhängigen und einen eher konjunkturunabhängigen Bereich gliedert, wobei Ersterer umsatztechnisch überwiegt. Diese Zweiteilung der Handwerkswirtschaft hinsichtlich des Ausmaßes der Konjunkturabhängigkeit wurde letztlich bereits am Beispiel der Wirtschaftskrise 2008/2009 deutlich (vgl. Abschnitt 3.2). Hieran zeigt sich, dass eine Aussage zur etwaigen Stabilisatorfunktion des Handwerks stets differenziert ausfallen muss.

Eine eindeutige Verneinung der Stabilisatorfunktion des Handwerks erscheint auch angesichts der stilisierten Fakten zur handwerklichen Beschäftigungsentwicklung fraglich. Hier zeigte sich, dass sich die Handwerke für den gewerblichen Bedarf und in abgeschwächter Form auch die Handwerke für personenbezogene Dienstleistungen prozyklisch und gleichlaufend zum gesamtwirtschaftlichen Konjunkturverlauf verhalten. Demgegenüber weisen jedoch verschiedene Gewerbegruppen des Handwerks zwar ebenfalls ein prozyklisches Beschäftigungsverhalten auf. Dieses läuft den Ausschlägen der gesamtwirtschaftlichen Erwerbstätigkeit jedoch mit einem Nachlauf von zwei bis vier Quartalen hinterher, was für ein vergleichsweise beharrendes Beschäftigungsverhalten im überwiegenden Teil des Handwerks spricht. Bei den Gesundheitshandwerken war darüber hinaus sogar ein antizyklisches Verhalten im Untersuchungszeitraum feststellbar. Dieser Befund kann somit als Indiz für eine zumindest bedingte Gültigkeit der Stabilisatorhypothese gelten.

Als Zwischenfazit bleibt festzuhalten, dass sich auch die Handwerkswirtschaft dem Auf und Ab der Gesamtwirtschaft nicht entziehen kann. Angesichts der sektoralen Verflechtung des Handwerks ist dies auch nicht weiter verwunderlich. Die überwiegende Konzentration des Handwerksumsatzes in eher konjunkturabhängigen Gewerbegruppen spricht in diesem Zusammenhang eher gegen eine Stabilisatorfunktion des Handwerks. Die aufgezeigten Konjunkturmuster zeigen aber auch, dass die Stabilisatorhypothese dennoch nicht ohne weiteres verworfen werden kann, wofür eine geringere Konjunkturabhängigkeit einzelner Gewerbegruppen (gemessen am Umsatz) und ein beharrendes Beschäftigungsverhalten in weiten Teilen des Handwerks spricht. Vor allem die Umsatz- und Beschäftigungsentwicklung in den Handwerken für den gewerblichen Bedarf steht dabei angesichts eines hohen Gleichlaufs zur Gesamtkonjunktur im Widerspruch zur Stabilisatorhypothese. Diese Sonderstellung innerhalb der Handwerkskonjunktur bringt Abbildung 7 mit Hilfe eines Kreuzkorrelogramms zum Ausdruck.

Es handelt sich um eine grafische Darstellung der zeitabhängigen Korrelationskoeffizienten zwischen den zyklischen Umsatz- und Beschäftigungsschwankungen im Handwerk. Umsätze werden hier als Präsensindikator und die Beschäftigung als Spätindikator der Handwerkskonjunktur verstanden. Folglich stellt sich die Frage, wie stark die aktuelle Beschäftigungslage im Handwerk die zeitlich vorgelagerte Umsatzentwicklung nachvollzieht, also inwiefern ein Nachlauf der Beschäftigung mit Bezug zur vorherigen Umsatzentwicklung gemessen werden kann. Hierbei zeigt sich, dass im Vergleich zum übrigen zulassungspflichtigen Handwerk nur bei den Handwerken für den gewerblichen Bedarf ein starker, prozyklischer Nachlauf zu beobachten ist. Ansonsten ist der Zusammenhang deutlich schwächer ausgeprägt, was auf ein stabileres Beschäftigungsverhalten deutet. Hinsichtlich der Erklärung dieses Befunds ist u.a. zu 
vermuten, dass z.B. handwerkliche Zulieferer der Industrie von „,industriellen“ Zulieferern kaum noch zu unterscheiden sind. Das Beschäftigungsverhalten der Industrie dürfte von diesen Handwerksbetrieben in der Folge weitgehend übernommen worden sein. ${ }^{54}$

Abbildung 7: Korrelationen zwischen Umsatz und Beschäftigung im Handwerk (Kreuzkorrelogramm; zyklische Komponenten; Quartalswerte; 1996-2009)

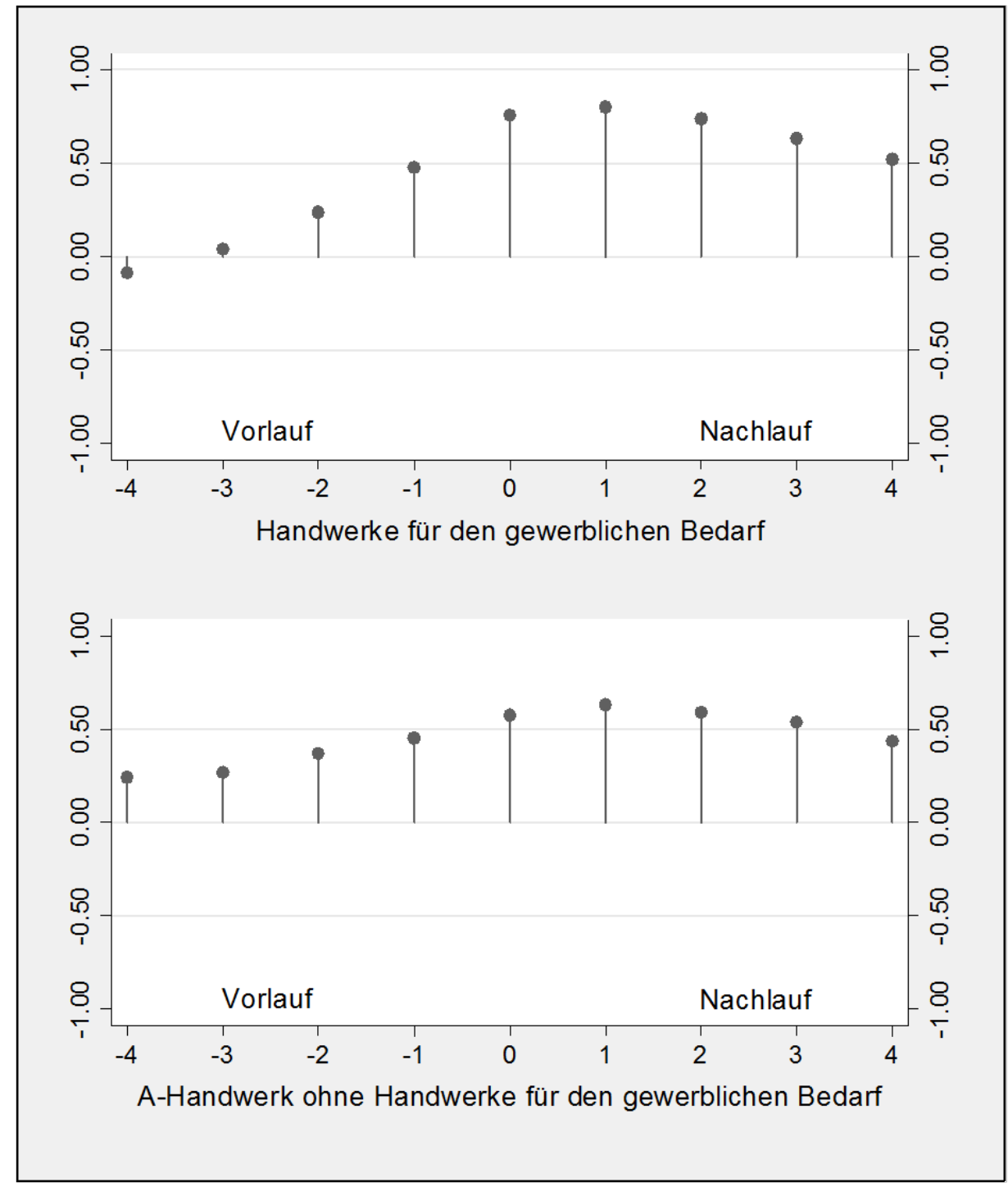

Quelle: Zentralverband des Deutschen Handwerks, Bundesbank, eigene Berechnungen

Abschließend bleibt an dieser Stelle zu betonen, dass die ermittelten Korrelationen zwischen Handwerks- und Gesamtwirtschaft noch keinen Rückschluss auf kausale Zusammenhänge hinsichtlich der gesamtwirtschaftlichen Verflechtung des Handwerks und der unterschiedlichen Konjunkturabhängigkeit von einzelnen Gewerbegruppen zulassen. Zur Einschätzung der eher proyzyklischen Umsatzschwankungen im Handwerk und somit auch mit Blick auf die Ausgangsfrage ist ebendies jedoch von besonderem Interesse. 


\section{Maßgebliche Determinanten der Handwerkskonjunktur}

\subsection{Bestimmungsfaktoren der Nachfrage nach Handwerksleistungen}

Am Beispiel von Abbildung 2 wurden zur Verdeutlichung der gesamtwirtschaftlichen Nachfrageschwäche im Zuge der Wirtschaftskrise 2008/2009 bereits die Entwicklungsverläufe von verschiedenen BIP-Verwendungskomponenten diskutiert. Angesichts der gesamtwirtschaftlichen Verflechtung des Handwerks beeinflussen die Schwankungen der volkswirtschaftlichen Nachfrageaggregate über drei Wege die konjunkturellen Ausschläge der Handwerkswirtschaft. Erstens unterscheiden sich die Verwendungskomponenten in ihrer grundsätzlichen Relevanz für das Handwerk. Hinsichtlich der Nachfrage des Auslandes nach deutschen Waren und Dienstleistungen ist diesbezüglich z.B. festzuhalten, dass sich der Exportumsatz im Handwerk seit 1995 zwar fast verdoppelt hat. Insgesamt betrug der Exportanteil am Gesamtumsatz des Handwerks im Jahr 2006 jedoch nur 3,5\%. ${ }^{55}$ Gemessen an direkten Einflüssen durch getätigte Auslandsgeschäfte hat die Entwicklung der volatilen Exportnachfrage folglich kaum einen Einfluss auf die konjunkturellen Ausschläge der Handwerkswirtschaft. Nennenswerte Anstöße durch den Export ergeben sich im Handwerk allenfalls auf indirektem Wege durch die Ausrüstungsnachfrage der Industrie (Handwerke für gewerblichen Bedarf) oder die Nachfrageentwicklung im Wirtschaftsbau (Bauhauptgewerbe). Zweitens wirken sich die konjunkturellen Schwankungen der besonders handwerksrelevanten BIP-Verwendungskomponenten, in Abhängigkeit von der Umsatzhöhe der hiervon jeweils betroffenen Gewerbegruppen, unterschiedlich stark auf die Wirtschaftslage des Gesamthandwerks aus. Drittens unterscheiden sich die Verwendungskomponenten selbst hinsichtlich ihrer konjunkturellen Dynamik. $^{56}$ Die einzelnen Nachfrageaggregate wirken dadurch entweder als „Zyklusverstärker“ oder als „Zyklusdämpfer“ der gesamtwirtschaftlichen Entwicklung, was wiederum konkrete Rückwirkungen auf die Konjunkturabhängigkeit der hiervon betroffenen handwerklichen Gewerbegruppen hat. Vor diesem Hintergrund ist aus konjunkturanalytischer Sicht eine Einteilung der Gewerbegruppen des Handwerks in drei (grobe) funktionale Gruppen und deren Vergleich mit den jeweils relevanten BIP-Verwendungskomponenten von besonderem Interesse. $^{57}$

Als erste funktionale Gruppe sind die Bau- und Ausbauhandwerke zu nennen. Mit einem Anteil am Gesamtumsatz in Höhe von 42 \% besitzt diese Gruppe das größte Gewicht innerhalb des zulassungspflichtigen Handwerks (vgl. Abbildung 6, S. 139). Hieran zeigt sich bereits die große Bedeutung der wirtschaftlichen Entwicklung im Baubereich für die Wirtschaftslage im Handwerk. Der Verlauf der gesamtwirtschaftlichen Baunachfrage lässt

\footnotetext{
55 Vgl. Müller, K. (2008), S. 60ff. Die Handwerke für den gewerblichen Bedarf (vor allem Feinwerkmechaniker) waren dabei am stärksten international tätig. Die meisten Handwerksbetriebe zeichnen sich jedoch weiterhin durch eine starke regionale Verankerung aus.

56 Vgl. Pätzold, J. und Baade, D. (2008), S. 49ff.

57 Vgl. Dürig, W. u. a. (2004), S. 82ff.
} 
sich hingegen am Umfang der Investitionen ablesen, die pro Jahr in den verschiedenen Bausparten (Wohnungsbau, Wirtschaftsbau, öffentlicher Bau) getätigt werden. Die Bauwirtschaft selbst wiederum ist stark durch das Handwerk geprägt. ${ }^{58}$ Aufgrund der hohen Absatzkonzentration dieser Handwerksgruppe erklärt sich folglich auch der Umstand, dass die zyklischen Schwankungen der Umsätze im Bau- und Ausbauhandwerk sehr stark mit denjenigen der Bauinvestitionen korrelieren und auch deren Volatilität im Zeitablauf ähnlich hoch ist (vgl. Abbildung 8). ${ }^{59}$ Die konjunkturelle Lage des Bau- und Ausbauhandwerks spiegelt folglich die Schwankungen der Baunachfrage deutlich wider.

Die Handwerke für den gewerblichen Bedarf bilden die zweite funktionale Handwerksgruppe. Mit einem Umsatzanteil von 18 \% besitzt dieser Bereich ein - relativ gesehen - geringes Gewicht innerhalb der Handwerkswirtschaft (vgl. Abbildung 6, S. 139). Die inländische Nachfrage nach beweglichen Investitionsgütern (Maschinen, maschinelle Anlagen, Geräte, Betriebs- und Geschäftsausstattungen etc.) kann am Verlauf der Ausrüstungsinvestitionen abgelesen werden. Als vorrangig zuliefernde Handwerke sind die Handwerke für den gewerblichen Bedarf wiederum in besonderem Maße von der volatilen Ausrüstungsnachfrage abhängig. Die zyklischen Schwankungen der Umsätze in dieser Gewerbegruppe verlaufen in der Folge in enger Korrelation zu denjenigen der Ausrüstungsinvestitionen. Im Vergleich zu den anderen funktionalen Handwerksgruppen bzw. den anderen Nachfrageaggregaten ist im Zuge dessen auch die Volatilität in beiden Fällen ähnlich hoch ausgeprägt (vgl. Abbildung 8). ${ }^{60}$

58 Neben dem fast vollständig handwerklich dominierten Ausbaugewerbe ist auch das Bauhauptgewerbe mit einem Anteil von knapp 70 \% der Betriebe und 73 \% der Beschäftigten stark durch das Handwerk geprägt (Stand 2008). Vgl. ZDB (2009), S. 25 und S. 68.

59 Nennenswerte Unterschiede ergeben sich im Beobachtungszeitraum nur aufgrund eines konjunkturellen Sondereffekts: Die Anhebung des Mehrwertsteuer-Regelsatzes auf 19 \% zum 1. Januar 2007 hat im Bauund Ausbauhandwerk zu besonders deutlichen Vorzieheffekten geführt.

60 Als Einschränkung sei an dieser Stelle nochmals angemerkt, dass sich die vorliegenden Ergebnisse nur auf das zulassungspflichtige Handwerk beziehen. Die zulassungsfreien B1-Handwerke für den gewerblichen Bedarf, zu denen z.B. das beschäftigungsstarke Gebäudereinigerhandwerk zählt, wurden daher nicht berücksichtigt. Gerade ein Dienstleistungshandwerk wie die Gebäudereiniger dürfte jedoch die Schwankungen der Ausrüstungsinvestitionen weniger stark nachvollziehen als z.B. handwerkliche Zulieferer der Industrie. Vgl. Dürig, W. u. a. (2004), S. 83f. 
Abbildung 8: BIP-Verwendungskomponenten und Umsätze in funktionalen Handwerksgruppen (zyklische Komponenten; Quartalswerte; 2000-2009)
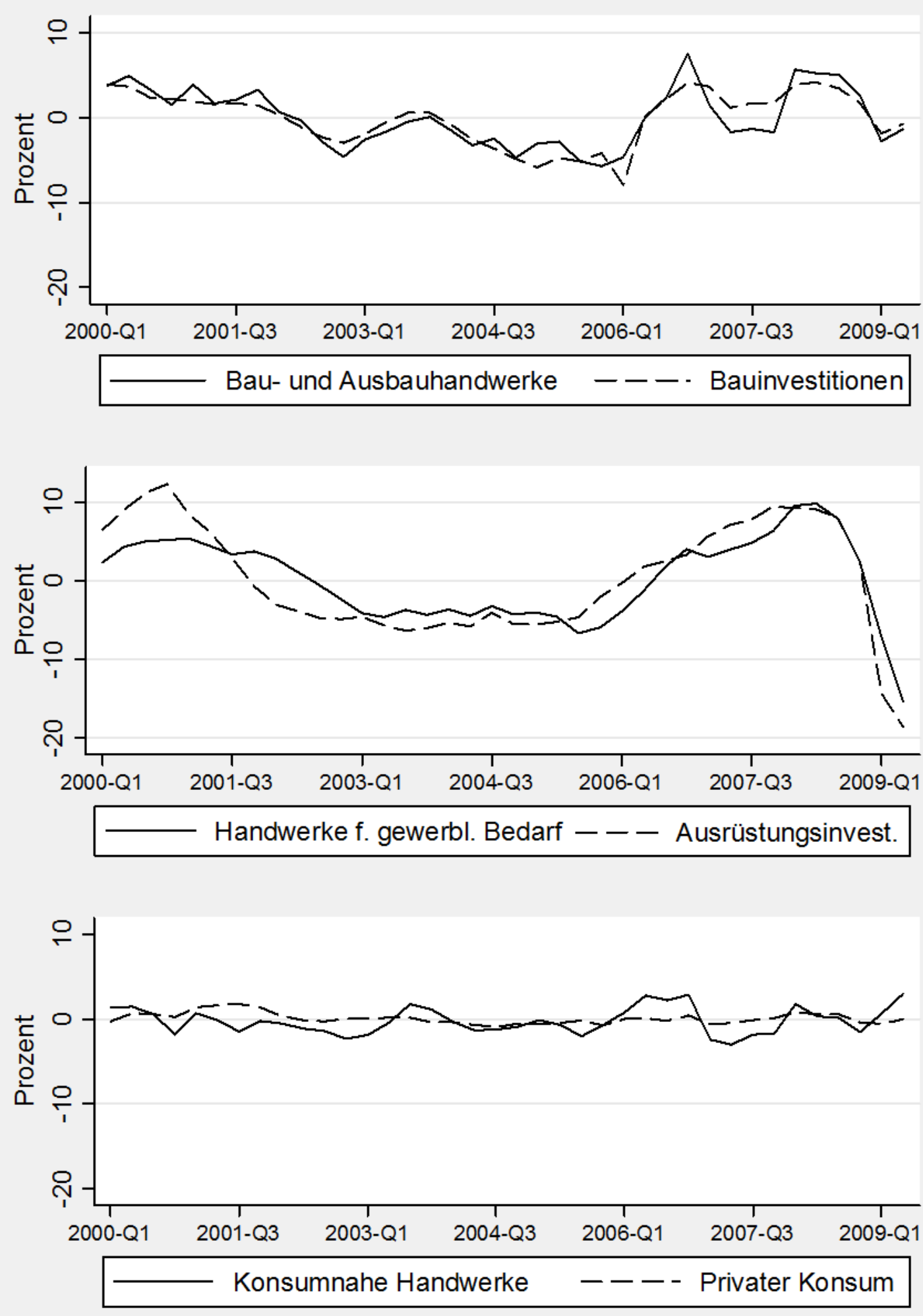

Quelle: Bundesbank, Zentralverband des Deutschen Handwerks, eigene Berechnungen 
Mit einem Umsatzanteil von 40 \% haben des Weiteren die konsumnahen Handwerke (Kfz-, Lebensmittel-, Gesundheits-, Personenbezogenes Dienstleistungsgewerbe) ein hohes Gewicht innerhalb der Handwerkswirtschaft. ${ }^{61}$ Die für diese Handwerksgruppe vor allem relevanten Konsumausgaben der privaten Haushalte werden in der Volkswirtschaftlichen Gesamtrechnung mit den Konsumausgaben der privaten Organisationen ohne Erwerbszweck zum privaten Konsum zusammengefasst. Bei Ersteren werden wiederum alle Waren- und Dienstleistungskäufe der inländischen Privathaushalte für Konsumzwecke erfasst, worunter folglich auch langlebige Gebrauchsgüter, wie z.B. Kraftfahrzeuge, gehören. Der private Konsum verläuft insgesamt proyzklisch und in etwa gleichlaufend zum Bruttoinlandsprodukt, wobei der Zusammenhang aber nur relativ schwach ausgeprägt und in beiden Fällen die Volatilität ähnlich niedrig ist. Absolut gesehen tragen die niedrigen Schwankungen des privaten Konsums jedoch aufgrund der Größe des Nachfrageaggregats - im Jahr 2008 betrug der Anteil am nominalen Bruttoinlandsprodukt $56 \%^{62}$ - in erheblichem Maße zum gesamtwirtschaftlichen Konjunkturgeschehen bei. Die relativ schwachen Konjunkturbewegungen des privaten Konsums sind dabei vorwiegend auf die im Aggregat dominierenden Konsumausgaben für nichtdauerhafte Güter und Dienstleistungen zurückzuführen. ${ }^{63}$ Die Käufe von langlebigen Gebrauchsgütern schwanken zwar in etwa dreimal so stark wie der gesamte private Konsum und sind folglich als eher konjunktursensibel zu bezeichnen. Aufgrund ihres geringen Anteils an den gesamten Konsumausgaben der privaten Haushalte wirkt sich dies in der Regel jedoch nur wenig auf den privaten Konsum als Ganzes aus. ${ }^{64}$

Vor diesem Hintergrund wird auch das zyklische Verlaufsmuster der konsumnahen Handwerke verständlich (vgl. Abbildung 8). Mit Blick auf die beiden anderen Vergleichspaare weist dieses eine ähnlich schwache Volatilität auf wie der private Konsum, wodurch bereits die hohe Bedeutung der privaten Konsumnachfrage für die konjunkturelle Lage der konsumnahen Handwerke zum Ausdruck kommt. Es wird jedoch auch deutlich, dass eine Korrelation zwischen den zyklischen Umsatzschwankungen dieser Handwerksgruppe mit denjenigen des privaten Konsums zwar vorhanden, aber nicht sehr stark ausgeprägt ist. Zudem fallen die zyklischen Schwankungen der konsumnahen Handwerke etwas höher aus als beim privaten Konsum. Dies dürfte auf verschiedene Umstände zurückzuführen sein. Erstens stellt sich im Falle der konsumnahen Handwerke die Verteilung der Konsumausgaben nach der Dauerhaftigkeit

61 Trotz der Einordnung des Kfz-Handwerks im konsumnahen Handwerksbereich muss beachtet werden, dass ein großer Teil der Kfz-Nachfrage auch auf gewerbliche Nutzer entfällt.

62 Vgl. Statistisches Bundesamt (2009), S. 77.

63 Im Jahr 2004 betrug der Anteil der langlebigen Konsumgüter an den inländischen Konsumausgaben der privaten Haushalte nur 11,3\%. Zu den nicht-dauerhaften Gütern und Dienstleistungen gehören neben kurzlebigen Gebrauchsgütern auch Verbrauchsgüter (z.B. Nahrungsmittel) und die einzelnen Dienstleistungsformen (z.B. Reparaturleistungen an Konsumgütern, Wohnungsmieten oder Beherbergungs- und Gaststättenleistungen). Vgl. Burghardt, M. (2006), S. 143.

64 Vgl. Tichy, G. (1994), S. 81ff. Die relativ starke Wirkung der durch die „Abwrackprämie“ angeregten Kraftfahrzeugkäufe auf die Entwicklung des privaten Konsums im Jahr 2009 dürfte diesbezüglich eine Ausnahme darstellen. Vgl. Abschnitt 3.1. 
der Güter deutlich anders dar als im volkswirtschaftlichen Vergleichsaggregat. Denn gemessen an der Umsatzhöhe dominiert das Kraftfahrzeuggewerbe innerhalb der konsumnahen Handwerke (vgl. Abbildung 6). Die Umsatzentwicklung in dieser Gewerbegruppe hängt wiederum stark vom konjunkturanfälligen Neu- und Gebrauchtwagenhandel $a b^{65}$, wodurch die Umsatzentwicklung der konsumnahen Handwerke insgesamt stärker von der schwankungsintensiveren Nachfrage nach langlebigen Konsumgütern beeinflusst sein dürfte als der private Konsum. Zweitens und hiermit eng zusammenhängend verschiebt sich innerhalb des privaten Konsums seit geraumer Zeit die Verteilung der Konsumausgaben zu Gunsten der Dienstleistungen. Allein zwischen 1991 und 2004 hat sich im Zuge dessen der Anteil der langlebigen Güter an allen Konsumausgaben der privaten Haushalte von 14,3 \% auf 11,3 \% kontinuierlich verringert. ${ }^{66}$ Der auch von Dürig u. a. (2004), S. 83 f. festgestellte Umstand, dass seit Ende der 90er Jahre der Zusammenhang zwischen der Umsatzentwicklung im konsumnahen Handwerk und dem privaten Konsum schwächer geworden ist, dürfte daher u.a. auch mit dieser Strukturverschiebung zusammenhängen. Drittens entfällt ein nicht unerheblicher Teil der Kfz-Nachfrage auf gewerbliche Nutzer, was ebenfalls zur geringeren Korrelation mit dem privaten Konsum beitragen dürfte. Vor dem Hintergrund dieses und des vorangegangenen Abschnitts erklärt sich somit die in Abschnitt 5.2 getroffene Feststellung, wonach bei den konsumnahen Gewerbegruppen des Handwerks ein eher schwacher Zusammenhang zum gesamtwirtschaftlichen Konjunkturverlauf besteht.

Die Betrachtung der handwerksrelevanten Nachfrageaggregate der Volkswirtschaftlichen Gesamtrechnung hat damit zugleich auch weitere Hinweise zur Beantwortung der Ausgangsfrage geliefert. Die obigen Ausführungen machten diesbezüglich deutlich, dass vom privaten Konsum in der Regel eine dämpfende bzw. stabilisierende Wirkung auf den Konjunkturverlauf ausgeht. Eine relativ stabile Entwicklung in diesem Bereich lässt darauf schließen, dass private Konsumausgaben weniger vom aktuell verfügbaren Einkommen, sondern eher von der durchschnittlichen Einkommensentwicklung abhängig sind. Kurzfristige zyklische Schwankungen des privaten Konsums sind daher weniger wahrscheinlich. ${ }^{67}$ Kann die private Konsumnachfrage somit in der Regel als „Zyklusdämpfer“ gelten, wird die private Investitionsnachfrage hingegen zu den „Zyklusverstärkern“ gezählt. Dies gilt vor allem für die Bruttoanlageinvestitionen, die sich gemäß der Volkswirtschaftlichen Gesamtrechnung überwiegend aus den diskutierten Bau- und Ausrüstungsinvestitionen zusammensetzen. Die Anla-

65 Im Jahr 2008 betrug der Anteil des Neu- und Gebrauchtwagenhandels am Gesamtumsatz des Kraftfahrzeuggewerbes 78\%. Im Kfz-Handwerk fällt dieser Anteil zwar vor allem aufgrund des Werkstattgeschäfts geringer aus. Dennoch spielt auch dort der Handel eine zentrale Rolle. In der Handwerkszählung 1994 entfielen knapp 68 \% des Gesamtumsatzes im Kfz-Technikerhandwerk auf Handelsgeschäfte. Vgl. ZDK (2009), S. 9 und Dürig, W. u. a. (2004), S. 245ff.

66 Vgl. Burghardt, M. (2006), S. 143.

67 Schwankungen der Konsumnachfrage können freilich dennoch großen Einfluss auf die konjunkturelle Gesamtentwicklung nehmen. Gemäß der Akzeleratorhypothese wird davon ausgegangen, dass bereits geringe Ausschläge des privaten Konsums zu überproportionalen Schwankungen der privaten Investitionstätigkeit führen. Vgl. Pätzold, J. und Baade, D. (2008), S. 51. 
geinvestitionen verlaufen weitgehend gleichlaufend und stark prozyklisch zum Bruttoinlandsprodukt, wobei jedoch bei Ersteren die Ausschläge zwei- bis viermal höher ausfallen. Hierbei gelten die Ausrüstungsinvestitionen als besonders konjunkturreagibel. Ein wichtiger Grund für den unsteten Verlauf der privaten Investitionsnachfrage wird darin gesehen, dass das Investitionsverhalten von Unternehmen vor allem in kurz- und mittelfristiger Perspektive - stärker als z.B. der private Konsum - von Erwartungen abhängig ist und auf Zinsänderungen, Lohnstückkosten sowie institutionelle Neuregelungen (Abschreibungsmodalitäten etc.) sensibel reagiert. Starke zyklische Schwankungen der Anlageinvestitionen haben dabei zum einen zur Folge, dass auch der Auslastungsgrad der gesamtwirtschaftlichen Produktionskapazitäten zyklischen Schwankungen unterworfen ist. Zum anderen unterliegt hierdurch auch die Zuwachsrate der Kapazitätsentwicklung einem zyklischen Verlaufsmuster. Die zyklisch schwankende Investitionstätigkeit ist daher stets ein wichtiger Ansatzpunkt für eine Politik der Konjunkturstabilisierung. ${ }^{68}$

An dieser Stelle bleibt folglich festzuhalten, dass verschiedene Aggregate der Volkswirtschaftlichen Gesamtrechnung als Bestimmungsfaktoren der Nachfrage nach Handwerksleistungen gelten können. Je stärker diese Verwendungskomponenten im Konjunkturverlauf schwanken und umso höher der jeweilige Umsatzanteil der hiervon betroffenen Handwerksgruppen ist, desto stärker ist auch der Einfluss auf die Konjunkturlage des Gesamthandwerks. Die gesamtwirtschaftlichen Nachfrageschwankungen übertragen sich dabei innerhalb des Handwerks vor allem auf die funktionalen Handwerksgruppen „Gewerblicher Bedarf“ sowie „Bau und Ausbau“, da deren Abnehmer ein eher prozyklisches Nachfrageverhalten aufweisen. Die konjunkturellen Schwankungen der gesamtwirtschaftlichen Bau- bzw. Ausrüstungsnachfrage spiegeln sich daher in der Umsatzentwicklung der Bau- und Ausbauhandwerke bzw. den Handwerken für den gewerblichen Bedarf deutlich wider. Die bereits in Abschnitt 5.2 festgestellte Konjunkturabhängigkeit der entsprechenden Gewerbegruppen (vor allem bei den Handwerken für den gewerblichen Bedarf) wird somit im gesamtwirtschaftlichen $\mathrm{Zu}$ sammenhang verständlich. Zugleich haben die obigen Ausführungen gezeigt, dass der private Konsum in der Regel als Zyklusdämpfer wirkt, woraus sich eine stabilisierende Funktion für den gesamtwirtschaftlichen Konjunkturverlauf ergibt. Angesichts dieser Tatsache erklärt sich auch die geringere Konjunkturabhängigkeit der konsumnahen Gewerbegruppen des Handwerks. Die Betrachtung der gesamtwirtschaftlichen Determinanten der Nachfrage nach Handwerksleistungen hat damit aber auch vor Augen geführt, dass in der Vergangenheit von der privaten Konsumnachfrage kaum konjunkturelle Impulse ausgegangen sind. Die konjunkturelle Dynamik der deutschen Wirtschaft wurde im Vorfeld der Wirtschaftskrise 2008/2009 statdessen vor allem durch umfangreiche Exportaktivitäten gespeist ${ }^{69}$, von denen das Handwerk insgesamt nur unterproportional profitieren konnte.

68 Vgl. Tichy, G. (1994), S. 81ff; Pätzold, J. und Baade, D. (2008), S. 51f; Duong, M. H. (2002), S. 638 .

69 Vgl. Loschky, A. und Ritter, L. (2007); Steinfelder, J. (2007). 
Letztlich wurde damit deutlich, dass die Betroffenheit des Handwerks von wirtschaftlichen Krisen stets von der Struktur der jeweils aktuellen gesamtwirtschaftlichen Nachfrageschwäche abhängt. Das folgende Kapitel reflektiert vor diesem Hintergrund den konjunkturellen Trendverlauf im Handwerk von der Mitte der 90er Jahre bis zur Wirtschaftskrise 2008/2009.

\subsection{Konjunkturelle Trendentwicklungen im Vorfeld der Krise}

Die Entwicklung der Handwerkswirtschaft von der Mitte der 90er Jahre bis zum Ausbruch der Wirtschaftskrise in der zweiten Hälfte des Jahres 2008 unterlag sowohl konjunkturellen als auch strukturellen Einflüssen. Von 1996 bis zum Jahr 2005 sanken die Umsätze und die Beschäftigung in den A-Handwerken kontinuierlich ab, wobei die Beschäftigungsverluste stärker ausgefallen sind (vgl. Abbildung 9). Ab dem Jahr 2006 legten die Handwerksumsätze wieder zu. Der etwas unregelmäßige Verlauf ergab sich hierbei durch Vorzieheffekte im Zuge der Mehrwertsteuererhöhung zum 1. Januar 2007. Die Beschäftigung stabilisierte sich in diesem Zeitraum. Erst das Krisenjahr 2009 beendete (vorerst) diese für das Handwerk positive Entwicklung (vgl. Abschnitt 3.2). Die Entwicklung der Handwerkskonjunktur seit der Mitte der 90er Jahre bis zum Ausbruch der Wirtschaftskrise im Jahr 2008 kann daher grob in die Zeitabschnitte 1996 bis 2005 und 2006 bis 2008 eingeteilt werden.

Abbildung 9: Umsatz- und Beschäftigungsentwicklung im zulassungspflichtigen Handwerk $(1996=100)$

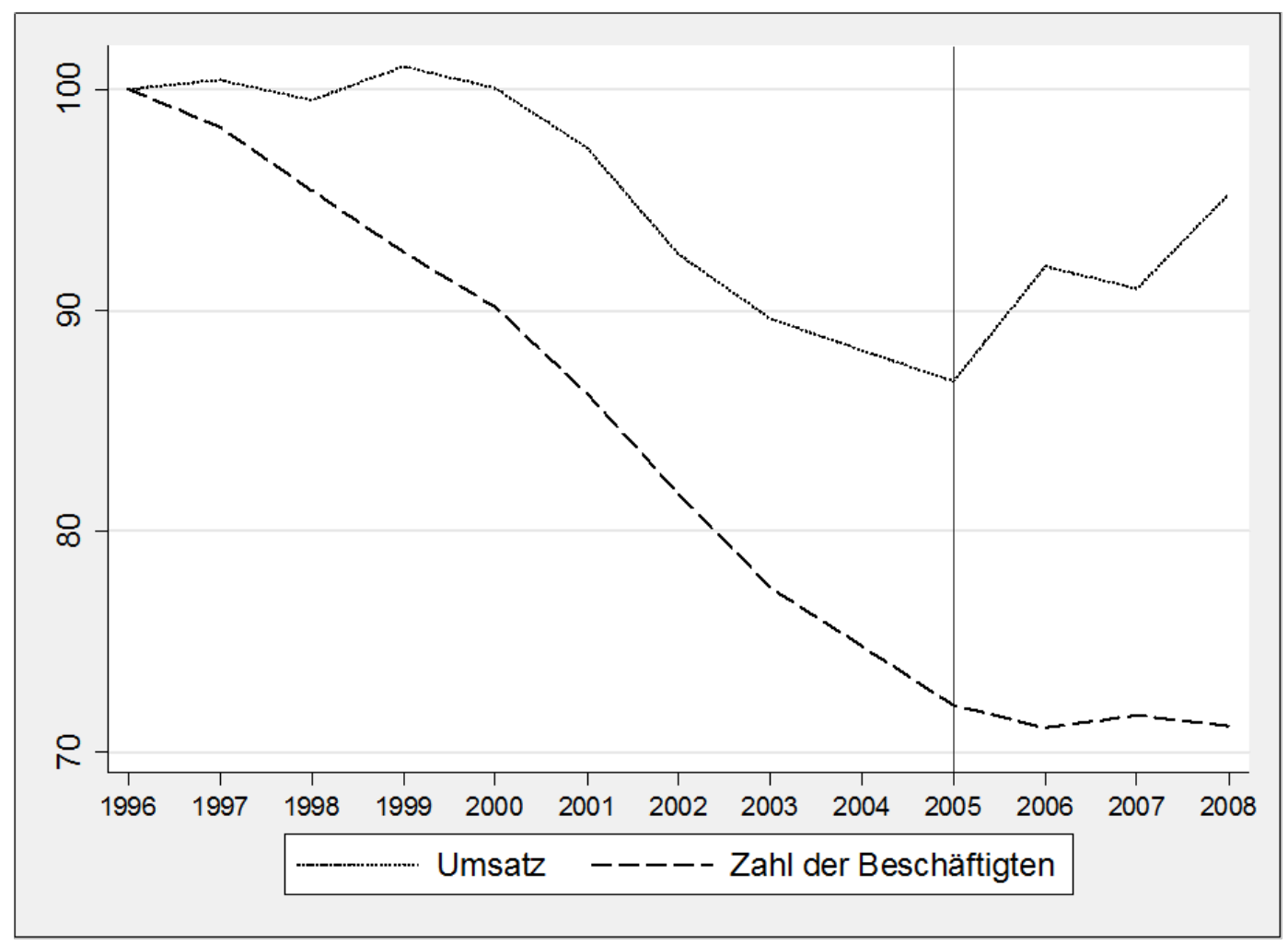

Quelle: Zentralverband des Deutschen Handwerks, eigene Berechnungen 
In den Jahren 1996 bis 2005 durchlief das Handwerk eine ausgeprägte Abschwungphase, die durch starke Umsatzrückgänge und Beschäftigungsverluste gekennzeichnet war. Die Bauund Ausbauhandwerke waren hierbei von den größten kumulierten Verlusten betroffen. Nach Schätzungen des RWI baute diese Handwerksgruppe allein zwischen 1996 und 2003 ca. 26 \% an Umsätzen und etwa 33 \% ihrer Beschäftigten ab. Die langjährige Baukrise kann daher als wichtigste Ursache für den allgemeinen Schrumpfungsprozess im Handwerk gelten. Mit Blick auf den Transformationsprozess in Ostdeutschland war diese Krise dabei eindeutig strukturell bedingt, da dem Wiedervereinigungsboom in der Bauwirtschaft ein notwendiger Abbau von Überkapazitäten ab den späten 90er Jahren folgte. Konjunkturell verstärkt wurde die Baukrise noch durch den zeitgleichen Eintritt der westdeutschen Bauwirtschaft in eine Abschwungphase im Rahmen ihres acht- bis zehnjährigen Bauzyklus. ${ }^{70}$ Gesamtwirtschaftliches Spiegelbild dieser Entwicklung war wiederum der Verlauf der Baunachfrage. Nach dem starken Anstieg nach der Wiedereinführung (+20,5\% im Jahr 1994) sanken die Zuwachsraten der realen Bauinvestitionen nahezu kontinuierlich ab auf -8,7 \% im Jahr 2005. ${ }^{71}$

Des Weiteren stellte sich die allgemeine konjunkturelle Lage der Handwerkswirtschaft im Zeitraum 2002 bis 2005 als besonders problematisch dar. ${ }^{72}$ Den Hintergrund hierfür lieferte eine parallel verlaufende gesamtwirtschaftliche Nachfrageschwäche. Bereits seit Mitte 2000 war die Konjunktur der deutschen Wirtschaft durch einen Wechsel von rezessiven und stagnativen Tendenzen gekennzeichnet. Im Jahr 2002 stagnierte die Wirtschaft (Nullwachstum) und erreichte im Rezessionsjahr 2003 eine reale Wachstumsrate von -0,2 \%. Zur gleichen Zeit brach die Handwerkskonjunktur auf breiter Front ein. ${ }^{73}$ Maßgeblich beigetragen zur Abkühlung der Konjunktur hatte die Verschlechterung der außenwirtschaftlichen Rahmenbedingungen, was insbesondere die stark exportorientierte deutsche Wirtschaft zu spüren bekam. Das Platzen der „Dotcom-Blase“, die Terroranschläge vom 11. September 2001, die Unsicherheit durch den Irak-Konflikt und eine Aufwertung des Euro lieferten zentrale Dämpfer der Konjunktur. Nachdem sich die Exportdynamik im Jahr 2002 merklich verlangsamt hatte und die Ausfuhren im Jahr 2003 sogar relativ stark gesunken waren, wirkte die Auslandsnachfrage aber bis zum Jahr 2005 als Stütze der schwächelnden Gesamtwirtschaft. Ein prägendes Merkmal der damaligen Wirtschaftslage war stattdessen die anhaltend schwache Binnennachfrage. $^{74}$

70 Vgl. Lageman, B. u. a. (2005), S. 4ff; Kornhardt, U. und Kucera, G. (2003), S. 108ff.

71 Vgl. ZDB (2007), S. 13. Nur im Jahr 1999 ergab sich noch ein leichtes Zwischenhoch (+1,5 \%).

72 Siehe hierzu auch Abbildung 3 und Abbildung 4.

73 Vgl. z.B. die folgenden ZDH-Konjunkturberichte: Ausgabe 2/2002 (Titel: „Konjunkturkrise im Handwerk zugespitzt“) und Ausgabe 1/2003 (Titel: „Handwerk ringt um Aufträge“). Im Frühjahr 2003 schätzte Creditreform (2003), S. 1 die Wirtschaftslage im Handwerk folgendermaßen ein: „Nahezu alle Wirtschaftsbereiche sind von der Konjunkturflaute betroffen, doch in diesem Jahr hat insbesondere das Handwerk auf seine schlechte wirtschaftliche Lage aufmerksam gemacht.“

74 Mit Bezug auf den folgenden Abschnitt vgl. ifo (2003), S. 16ff; ifo (2004),S. 18ff; ifo (2005), S. 21ff. Zum Überblick vgl. auch Abbildung 2. 
Die binnenwirtschaftliche Nachfrageschwäche dieser Jahre zeigte sich bereits am obigen Beispiel der kontinuierlich sinkenden Baunachfrage. Neben den starken Rückgängen im Wohnungsbau kam in diesen Jahren hinzu, dass sich die öffentlichen Bauinvestitionen angesichts einer angespannten kommunalen Haushaltslage verringerten und die Nachfrage im Wirtschaftsbau ebenfalls bis zum Jahr 2005 rückläufig war. Angesichts dieses breiten Rückgangs der Baunachfrage erklärt sich die besonders kritische Lage der Bau- und Ausbauhandwerke in dieser Zeit (vgl. Abbildung 10). Parallel dazu durchlief aufgrund des eingetrübten Investitionsklimas auch die Nachfrage nach Ausrüstungsgütern seit 2001 eine fortwährende und nur von leichten Erholungstendenzen unterbrochene Schwächephase, deren Ende sich erst im Jahr 2004 allmählich abzeichnete. Die konjunkturelle Entwicklung der Handwerke für den gewerblichen Bedarf hat diese Entwicklung wiederum deutlich nachvollzogen (vgl. Abbildung 10). Zusätzlich hierzu war die Zeit seit 2002 auch durch eine anhaltende Konsumschwäche geprägt. Der private Konsum entwickelte sich außerordentlich schwach und stagnierte mehr oder weniger auf niedrigem Niveau. Die Zurückhaltung der privaten Konsumenten in dieser Zeit macht daher die - relativ zu anderen Jahren - kritische Lage der konsumnahen Handwerke zwischen 2002 und 2005 verständlich (vgl. Abbildung 10).

Abbildung 10: Konjunkturelle Trendentwicklung von funktionalen Handwerksgruppen im Vergleich zum BIP (nominale Umsätze; 1996 bis 2009; 1996 = 100)

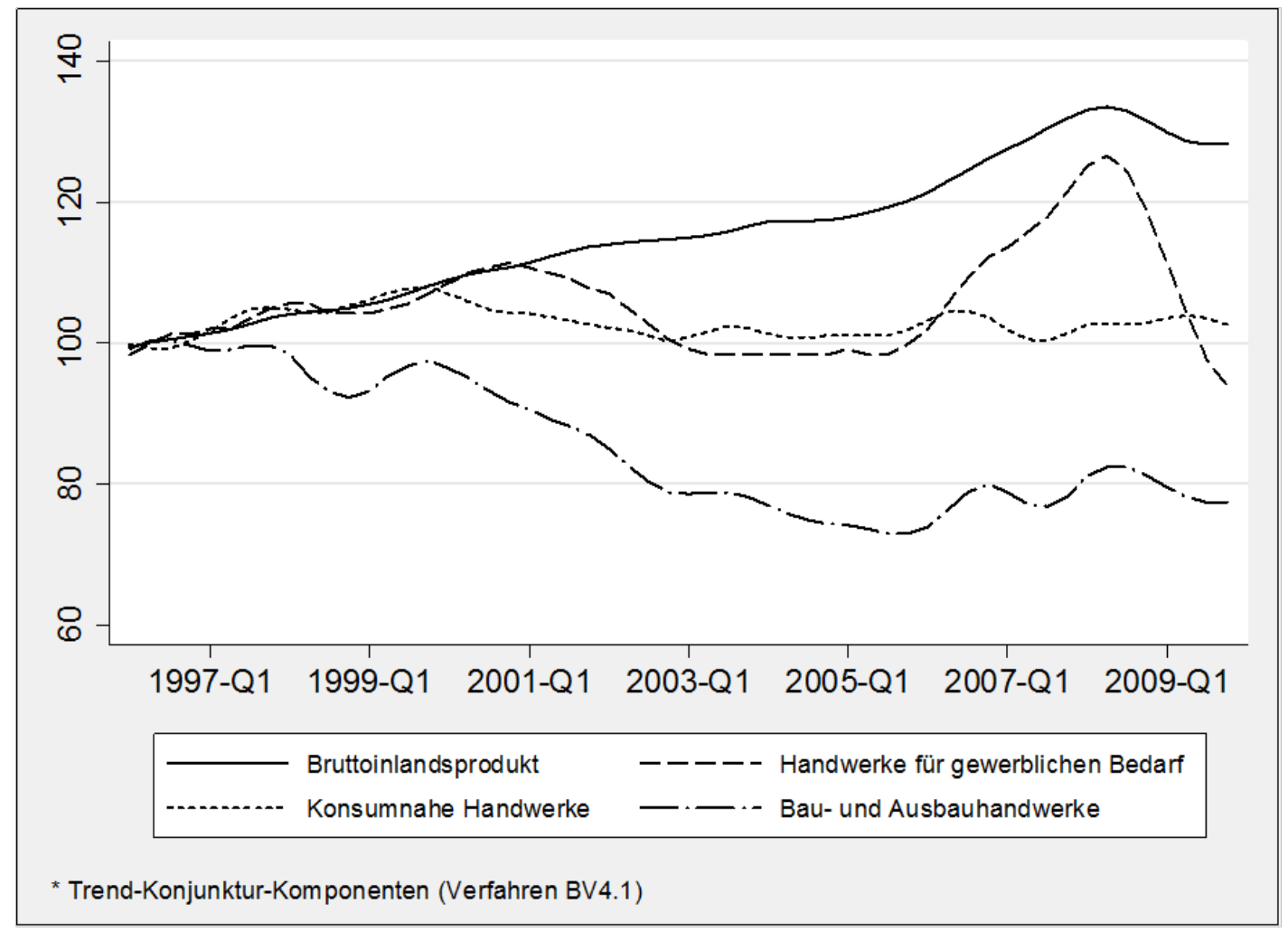

Quelle: Zentralverband des Deutschen Handwerks, eigene Berechnungen

Alles in allem wird somit deutlich, wieso mit Blick auf die Zeitperiode 2002 bis 2005 zuweilen von den „Krisenjahren des Handwerks“ gesprochen wird. Die Struktur der damaligen gesamtwirtschaftlichen Nachfrageschwäche führte dazu, dass das Handwerk in seiner Gänze 
von einem Konjunktureinbruch betroffen war und in der Gesamtschau somit auch nicht einzelne Krisenherde aufgrund der großen Heterogenität der Handwerkskonjunktur kompensiert werden konnten. Die ungünstigere Entwicklung des Handwerks im Vergleich zur Gesamtwirtschaft ist in dieser Phase daher neben strukturellen Faktoren zudem auf eine nachteilige konjunkturelle Konstellation zurückzuführen. ${ }^{75}$

In der Phase 2006 bis 2008 wurde auch das Handwerk vom allgemeinen Konjunkturaufschwung der deutschen Wirtschaft erfasst. Nachdem erste Tendenzen einer Erholung bereits seit dem Jahr 2004 zu spüren waren, gewann die Konjunktur ab 2006 deutlich an Fahrt. Der Konjunkturaufschwung wurde hierbei angesichts einer kräftigen Expansion der Weltwirtschaft vor allem von der rasch wachsenden Exportgüternachfrage getrieben. Zudem war die Konjunktur insofern nicht mehr gespalten, da nun auch von der Binnennachfrage Wachstumsimpulse ausgingen. ${ }^{76}$ Die Nachfrage der Unternehmen nach Ausrüstungsgütern legte angesichts positiver Absatz- und Gewinnerwartungen kräftig zu. Allein im Durchschnitt des Jahres 2006 wuchsen die Ausrüstungsinvestitionen um 7,3 \%, was der stärkste Anstieg seit dem Jahr 2000 war. Neben den Exporten wurden damit die Ausrüstungsinvestitionen bis in das erste Halbjahr 2008 hinein zum Wachstumstreiber der deutschen Wirtschaft. Entsprechend verzeichneten die Handwerke für den gewerblichen Bedarf im gleichen Zeitraum einen starken Anstieg ihrer Umsätze, wodurch diese Gewerbegruppe zur Konjunkturlokomotive des Handwerks wurde (vgl. Abbildung 10).

Ebenfalls mit dem Jahr 2006 endete zudem der über zehn Jahre andauernde Abwärtstrend der Baunachfrage, da nun die Bauinvestitionen in allen drei Bausparten wieder zulegten. ${ }^{77}$ Die Nachfrage der Unternehmen nach gewerblichen Bauten (Wirtschaftsbau) stieg im Zuge des allgemeinen Investitionsbooms zwischen 2006 und 2008 deutlich an. Vom Wohnungsbau gingen im Jahr 2006 noch starke Impulse aus, danach bremsten aber die Mehrwertsteuererhöhung und der Wegfall der Eigenheimzulage die dortige Gesamtentwicklung. Aus Sicht des Handwerks ist hierbei jedoch hervorzuheben, dass trotz der insgesamt eher schwachen Entwicklung im Wohnungsbau von energetischen Bestandssanierungen fortwährend wichtige nachfragewirksame Impulse ausgegangen sind. Angesichts eines kräftigen Anstiegs der Aufträge durch öffentliche Auftraggeber (Abbau von angestautem Baubedarf) und einer stark verbesserten Finanzsituation in den Kommunen gingen in den Jahren 2006 bis 2008 des Weiteren auch vom öffentlichen Bau starke Impulse aus. Vor diesem Hintergrund ist die konjunkturelle Lage der Bau- und Ausbauhandwerke in dieser Zeit zu sehen, deren Umsätze mit dem

75 Dispan, J. (2003), S. 118ff.

76 Mit Bezug auf diesen und die folgenden beiden Abschnitte vgl. ifo (2006), S. 16ff; ifo (2007), S. 19ff; ifo (2008), S. 27ff. Zum Überblick vgl. auch Abbildung 2.

77 Die Zunahme der Bauinvestitionen im Jahr 2006 war jedoch nicht nur konjunkturell bedingt. Aufgrund einer turnusmäßigen Überarbeitung der Ergebnisse der Volkswirtschaftlichen Gesamtrechnung stellte sich der Zuwachs etwas überzeichnet dar. Vgl. ZDB (2007), S. 13. 
Jahr 2006 nach langer Zeit wieder auf einen leichten Wachstumspfad eingeschwenkt waren (vgl. Abbildung 10).

Auch der private Konsum hatte im Verlauf des Jahres 2006 merklich zugenommen. Neben Realeinkommenszuwächsen hatten insbesondere Vorzieheffekte im Vorfeld der Mehrwertsteuererhöhung dazu geführt, dass die Nachfrage nach langlebigen Gebrauchsgütern, wie z.B. Pkw, anstieg. Im Jahr 2007 erlitt der private Konsum dann aufgrund der vorgezogenen Käufe und des Kaufkraftentzugs in Folge der steuerbedingt erhöhten Verbraucherpreise einen Dämpfer. Trotz einer leichten Belebung konnte der private Konsum damit bis in das Jahr 2008 hinein keine wirkliche Dynamik entfalten. Vor diesem Hintergrund ist auch die Entwicklung der konsumnahen Handwerke in den Jahren 2006 bis 2008 zu sehen. Deren Lage stellte sich zwar eher schwach, aber relativ zu den vorangegangenen Krisenjahren dennoch etwas besser dar (vgl. Abbildung 10).

Im Vergleich zur vorherigen Phase zeichnete sich der Zeitabschnitt 2006 bis 2008 somit durch eine gesamtwirtschaftliche Nachfragesituation aus, in der auch von der Inlandsnachfrage konjunkturelle Anstöße ausgegangen sind. Hiervon konnte wiederum das binnenmarktorientierte Handwerk stark profitieren. Strenggenommen hat die Handwerkswirtschaft in dieser Phase daher freilich nur bedingt zur Glättung des gesamtwirtschaftlichen Konjunkturverlaufs beigetragen (vgl. Abbildung 4, S. 131). Mit Blick auf die Ausgangsfrage zeigt der Vergleich dieser beiden Zeitperioden damit folgendes: Eine konjunkturelle Stabilisatorfunktion kann sich im Falle des Handwerks zwar aufgrund einer relativ geringen Abhängigkeit vom volatilen Exportgeschäft ergeben. ${ }^{78}$ In exportinduzierten Wirtschaftskrisen trägt das wirtschaftliche Geschehen im Handwerk damit auch eher zur Stützung des gesamtwirtschaftlichen Konjunkturverlaufs bei. Zur Erfüllung einer solchen Stabilisatorfunktion ist es aber notwendig, dass sich die Inlandsnachfrage entsprechend stabil entwickelt. Eine schwache Binnennachfrage, wie sie z.B. in den Jahren 2002 bis 2005 zu beobachten war, führt dazu, dass das Handwerk eine solche Funktion kaum erfüllen kann.

Zum Abschluss begründet sich damit auch die stabilisierende Wirkung weiter Teile des Handwerks in der Wirtschaftskrise 2008/2009. Wie in Abschnitt 3.1 ausgeführt, griff die weltweite Finanz- und Wirtschaftskrise vor allem durch einen massiven Einbruch der Exporte auf die deutsche Realwirtschaft über. Die sich hieraus ergebenden Multiplikatorprozesse wirkten sich des Weiteren vornehmlich auf die konjunktursensible private Investitionsnachfrage aus, was sich in deutlichen Rückgängen der Nachfrage nach Ausrüstungsgütern und gewerblichen Bauten äußerte. Die Handwerke für den gewerblichen Bedarf wurden von dieser Entwicklung aufgrund ihrer großen Industrienähe voll erfasst (vgl. Abbildung 10). Trotz des historisch einmaligen Einbruchs der Gesamtwirtschaft blieben jedoch im Krisenjahr 2009 - nicht zuletzt

78 Den überwiegenden Teil des Handwerks (und darüber hinaus das Gros der kleinen und mittleren Unternehmen) erreichen exportinduzierte Impulse allenfalls zeitverzögert und in abgeschwächter Form über Lieferund Leistungsverflechtungen sowie gesamtwirtschaftliche Nachfrageeffekte. Vgl. KfW u. a. (2003), S. 9f. 
auch aufgrund verschiedener konjunkturpolitischer Maßnahmen - die Inlandsnachfrage und damit auch weite Teile des Handwerks in der Summe relativ stabil. Die geringe Exportabhängigkeit der Handwerkswirtschaft dürfte sich daher in der Wirtschaftskrise 2008/2009 (ausnahmsweise) gleich doppelt zum Vorteil für das Handwerk ausgewirkt haben: Erstens war das binnenmarktorientierte Handwerk vom massiven Einbruch der Exportnachfrage deutlicher weniger betroffen als andere Wirtschaftsbereiche. Zweitens zielen konjunkturpolitische Maßnahmen auf eine Stimulierung der binnenwirtschaftlichen Nachfrage, weshalb Konjunkturpakete die Folgen eines weltweiten Produktionsrückgangs und eines Zusammenbruchs des Welthandels allenfalls mildern können. ${ }^{79}$ Das Handwerk dürfte daher von den fiskalpolitischen Maßnahmen der Konjunkturpakete - relativ gesehen - deutlich stärker profitiert haben als die exportorientierte Industrie. ${ }^{80}$

\subsection{Ausblick: Eine zukünftige Stabilisatorfunktion des Handwerks?}

Auf Grundlage der dargestellten Ergebnisse stellt sich die Frage, inwieweit zukünftig eher eine stabilisierende Wirkung des Handwerks zu erwarten ist. Es wurde deutlich, dass die diesbezüglichen Grundvoraussetzungen eine stabile Entwicklung der Inlandsnachfrage und eine geringe Exportabhängigkeit sind. Hierbei zeigte sich wiederum, dass durch den fortwährend hohen Anteil des Bau- und Ausbaugewerbes am Gesamtumsatz des Handwerks, das relative geringe Gewicht der industrienahen Handwerke und die eher stagnativen Tendenzen in den konsumnahen Gewerbegruppen das Auf und Ab der Handwerkskonjunktur letztlich vor allem von den zyklischen Schwankungen in der Bauwirtschaft bestimmt wird. Die Einschätzung von Beckermann (1974), wonach „die konjunkturellen Bewegungen im Handwerk in erster Linie vom Baugeschehen [...] bestimmt werden“" ${ }^{81}$ hat damit trotz der zurückliegenden Umbrüche in diesem Handwerksbereich immer noch ihre Gültigkeit.

Die hohe Abhängigkeit von der inländischen Baunachfrage und die geringe Abhängigkeit vom unmittelbaren Außenhandel sind folglich zentrale Determinanten einer etwaigen Stabilisatorfunktion des Handwerks. Mit Blick auf die entsprechenden Verwendungskomponenten zeigt die Entwicklung des Bruttoinlandsprodukts seit Anfang der 90er Jahre eine zunehmend deutlichere Verlagerung der hiermit verbundenen Wachstumskräfte. Spätestens mit Beginn des Jahrzehnts 2000-2009 gingen die (positiven und negativen) Wachstumsbeiträge in der deutschen Wirtschaft in immer stärkerem Maße vom Außenhandel aus. Im Vergleich dazu verharrte die Baunachfrage hingegen auf einem geringen Wachstumsniveau (vgl. Abbildung

\footnotetext{
79 Vgl. ifo (2009a), S. 75ff.
}

80 Dies lässt freilich noch keinen Rückschluss auf eine hohe inländische Wirksamkeit von fiskalpolitischen Maßnahmen zu. Gerade bei Volkswirtschaften mit hohem Offenheitsgrad wie der deutschen ergeben sich relativ starke Sickerverluste von fiskalpolitischen Maßnahmen ins Ausland. Vgl. hierzu Roos, M. W. M. (2009). Zum Zeitpunkt der Manuskriptfertigstellung war des Weiteren nicht im Detail bekannt, wie stark einzelne Bereiche des Handwerks von den Konjunkturpaketen profitiert haben. 
11). ${ }^{82}$ Angesichts der hiermit verbundenen Anfälligkeit für weltweite Konjunkturschwankungen führte die Wirtschaftskrise 2008/2009 eindrücklich vor Augen, welche Nachteile mit der überdurchschnittlich hohen Exportabhängigkeit der deutschen Volkswirtschaft verbunden sein können. Infolgedessen entwickelte sich im Zuge der Krise eine intensive Debatte um die deutsche Exportorientierung, in der von einigen Experten die deutsche Außenwirtschaftsstrategie als zu einseitige Fokussierung kritisiert wurde. ${ }^{83}$

Abbildung 11: Beiträge der Bauinvestitionen und des Außenbeitrags zum Wachstum des BIP (preis- und saisonbereinigt; 1992 bis 2009)

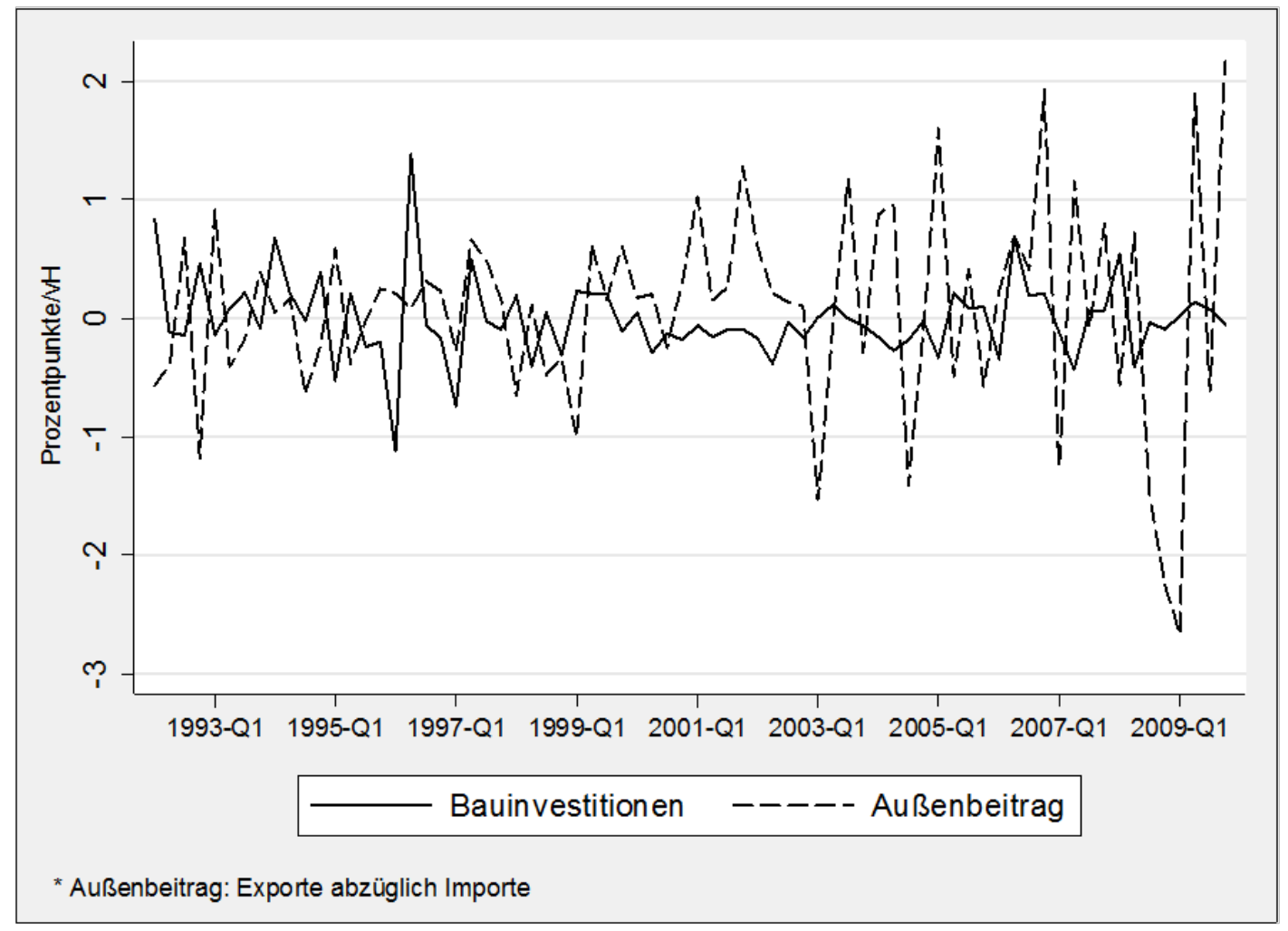

Quelle: Sachverständigenrat

Ungeachtet möglicher wirtschaftspolitischer Maßnahmen zur Erzielung einer ausgewogeneren Nachfragestruktur der deutschen Wirtschaft dürften jedoch angesichts der vergangenen Entwicklung auch zukünftig vom Export deutlich höhere Wachstumsimpulse ausgehen als bspw. von der inländischen Baunachfrage. Hieran wird sehr gut deutlich, dass eine etwaige Stabilisatorfunktion des Handwerks stets zwei Seiten hat. Da sich erstens an der geringen (unmittelbaren) Exportabhängigkeit des Handwerks im Wesentlichen auch in Zukunft nichts ändern dürfte, wird die Handwerkswirtschaft weiterhin von exportinduzierten Wirtschaftskrisen

82 Gleichwohl gehörte eine zunehmende Weltmarktverflechtung von jeher zu den Grundlinien der bundesdeutschen Wirtschaftsgeschichte. Vgl. Abelshauser, W. (2004), S. 217ff und 262ff.

83 Vgl. Horn, G. u. a. (2010). Siehe beispielhaft die folgenden Diskussionsbeiträge von Ökonomen im Handelsblatt: „Weltmeisterschaft auf Pump“ von Hans-Werner Sinn, in: Handelsblatt Nr. 174 vom 10.09.2009, S. 6 und „Ein Rückzug wäre fatal“ von Renate Ohr, in: Handelsblatt Nr. 129 vom 09.07.2009, S. 6. 
weniger stark betroffen sein als andere Wirtschaftsbereiche und in solchen Fällen somit eher als konjunktureller Stabilisator der Volkswirtschaft wirken. Letzteres dürfte künftig umso eher gelten, da im Zuge des demografischen Wandels von der besonders handwerksrelevanten Baunachfrage in der Summe kaum noch umfangreiche gesamtwirtschaftliche Wachstumsimpulse zu erwarten sind. ${ }^{84}$ Zweitens bringt die konjunkturstabilisierende Funktion aufgrund einer geringen Exportabhängigkeit für die Handwerkswirtschaft auch Schattenseiten mit sich. Denn aufgrund einer solchen Rolle dürfte das Handwerk weiterhin nur unterproportional von exportgetragenen Konjunkturaufschwüngen profitieren. Eine zukünftige Stabilisatorfunktion würde in diesem Falle somit bedeuten, dass die Handwerkswirtschaft im Wesentlichen von wichtigen Wachstumsbereichen der deutschen Wirtschaft abgekoppelt bleibt.

\section{Zusammenfassung und Implikationen}

Auch das Handwerk konnte sich den Auswirkungen der schweren Wirtschaftskrise 2008/2009 nicht entziehen. Prozentual gesehen fiel der Rückgang des nominalen Handwerksumsatzes im Jahr 2009 sogar stärker aus als im Falle des nominalen Bruttoinlandsprodukts. Zuvor war im Jahresverlauf hingegen von unterschiedlicher Seite immer wieder die konjunkturstabilisierende Wirkung des Handwerks hervorgehoben worden. Dies nicht zuletzt deshalb, da die im Rahmen von Konjunkturumfragen festgestellte Lage vieler Handwerksbetriebe im Vergleich zu anderen Wirtschaftsbereichen relativ stabil geblieben war. Der vorliegende Beitrag hat sich mit diesem Spannungsverhältnis auseinandergesetzt, indem er der Frage nachgegangen ist, inwiefern das Handwerk eine konjunkturelle Stabilisatorfunktion besitzt und welche wirtschaftspolitischen Folgerungen sich hieraus ergeben.

Die Untersuchung führte zu folgenden Ergebnissen:

- Der relativ starke Rückgang des Handwerksumsatzes im Jahr 2009 lässt zunächst darauf schließen, dass das Handwerk nicht als Stabilisator wirken konnte. Zwei Gründe sprechen dennoch dafür, dass große Teile des Handwerks in der Wirtschaftskrise 2008/2009 durchaus stabilisiert haben:

- Erstens hätten sich die summierten Handwerksumsätze im Jahr 2009 ohne die Handwerke für den gewerblichen Bedarf, worunter die von der Krise besonders stark in Mitleidenschaft gezogenen handwerklichen Zulieferer fallen, erheblich stabiler entwickelt.

- Zweitens erlebte die Handwerkswerkskonjunktur im Jahr 2009 zwar insgesamt - gemessen an der prozentualen Abweichung vom längerfristigen Trendverlauf - einen deutlichen Rückgang, dennoch war kein Einbruch wie in der Gesamtwirtschaft zu verzeichnen.

84 Vgl. Dürig, W. u. a. (2004), S. 272 f. Dessen ungeachtet bieten freilich einzelne Bereiche der Baunachfrage auch zukünftig für das Handwerk ein hohes Wachstumspotenzial, wie z.B. die energetische Sanierung und Modernisierung im Gebäudebestand. 
- Die Handwerkswirtschaft teilt sich in einen eher konjunkturabhängigen und einen weniger konjunkturabhängigen Bereich. Ersterer überwiegt hierbei umsatztechnisch, was gegen eine generelle Stabilisatorfunktion des Handwerks spricht. Die höchste Konjunkturabhängigkeit zeigen die Handwerke für den gewerblichen Bedarf. Relativ gesehen stärker fällt zudem die Konjunkturabhängigkeit im Falle der Bau- und Ausbaugewerbe aus. Die konsumnahen Gewerbegruppen des Handwerks erweisen sich demgegenüber als deutlich weniger konjunkturabhängig. Hieran bestätigt sich die bereits am Beispiel der Wirtschaftskrise 2008/2009 gemachte Beobachtung, dass eine Aussage zur etwaigen Stabilisatorfunktion des Handwerks stets differenziert ausfallen muss.

- Das charakteristische Beschäftigungsverhalten des überwiegenden Teils des Handwerks spricht eher für die Stabilisatorhypothese. Im Vergleich zur Gesamtwirtschaft weist der Großteil des Handwerks ein eher beharrendes Beschäftigungsverhalten auf. In der Summe halten demnach viele Handwerksbetriebe auch in konjunkturell schlechten Zeiten so lange wie möglich an ihren Mitarbeitern fest. ${ }^{85}$ Angesichts eines starken Gleichlaufs zum Auf und Ab der gesamtwirtschaftlichen Erwerbstätigkeit zeigte sich jedoch auch in diesem Fall die Sonderrolle der Handwerke für den gewerblichen Bedarf.

- Die Betroffenheit des Handwerks von wirtschaftlichen Krisen hängt stets von der Struktur der jeweils aktuellen volkswirtschaftlichen Nachfrageschwäche ab. Dies ist der Tatsache geschuldet, dass die unterschiedliche Konjunkturabhängigkeit der einzelnen Handwerksbereiche im Wesentlichen ein Spiegelbild der gesamtwirtschaftlichen Nachfrageentwicklung ist.

- Die Schwankungen der Handwerkskonjunktur werden in erster Linie von der Baunachfrage und in abgeschwächter Form von der privaten Konsumnachfrage bestimmt. Ein nennenswerter Einfluss auf die wirtschaftliche Lage im Gesamthandwerk geht zudem von der Nachfrage nach Ausrüstungsgütern aus. Abgesehen von indirekten Impulsen spielt demgegenüber die konjunktursensible Exportnachfrage für die Ausschläge der Handwerkskonjunktur nur eine untergeordnete Rolle. Vor diesem Hintergrund wird die aufgezeigte Konjunkturanfälligkeit der handwerklichen Gewerbegruppen verständlich. Denn im Falle der privaten Investitionsnachfrage (Bau und Ausrüstung) handelt es sich um besonders schwankungsintensive Verwendungskomponenten des Bruttoinlandsprodukts, wodurch sie eher als Zyklusverstärker der gesamtwirtschaftlichen Konjunkturentwicklung wirken. Hierdurch erklärt sich wiederum die höhere Konjunkturabhängigkeit der entsprechenden Gewerbegruppen (Bau, Ausbau und vor allem Gewerblicher Bedarf). Die private Konsumnachfrage dämpft hingegen in der Regel die konjunkturellen Ausschläge des Bruttoinlandsprodukts, weshalb die konsumnahen Handwerke eher stabilisierend wirken.

- Vor diesem Hintergrund erklärt sich die Betroffenheit des Handwerks in der Wirtschaftskrise 2008/2009: Die Gesamtwirtschaft wurde in der Krise vor allem durch einen Export-

85 Das eher konservative Beschäftigungsverhalten der Handwerksbetriebe führt freilich auch dazu, dass Neueinstellungen in konjunkturellen Aufschwungphasen eher zögerlich erfolgen. 
einbruch sowie durch deutliche Rückgänge der Nachfrage nach Ausrüstungsgütern und gewerblichen Bauten in Mitleidenschaft gezogen. Abgesehen davon blieb die Binnennachfrage - auch aufgrund erheblicher konjunkturpolitischer Eingriffe - weitgehend stabil. Die Handwerkswirtschaft wiederum ist insgesamt gesehen kaum exportabhängig. Des Weiteren spielen die im Krisenjahr 2009 besonders stark betroffenen Handwerke für den gewerblichen Bedarf für die Ausschläge der Handwerkskonjunktur eine weniger große Rolle als andere Handwerksbereiche. Die konjunkturelle Lage blieb daher in weiten Teilen des binnenmarktorientierten Handwerks vergleichsweise stabil. Die geringe Exportabhängigkeit des Handwerks dürfte dabei in zweierlei Hinsicht die relative Stabilität der Handwerkswirtschaft begünstigt haben: Erstens spielt der unmittelbare Exportumsatz in Folge von getätigten Auslandsgeschäften im Handwerk kaum eine Rolle. Zweitens stimulieren Konjunkturpakete vor allem die Inlandsnachfrage, wovon das Handwerk relativ gesehen - stärker profitiert haben dürfte als die exportorientierte Industrie.

Zusammengefasst lässt sich vor dem Hintergrund der dargestellten Ergebnisse die Ausgangsfrage nach der konjunkturellen Stabilisatorfunktion des Handwerks folgendermaßen beantworten:

1. Gemessen an der Konjunkturabhängigkeit der Umsatzentwicklung besitzt das Handwerk keine generelle Stabilisatorfunktion. In Abhängigkeit vom Charakter der jeweils aktuellen konjunkturellen Gesamtkonstellation kann ein solches Phänomen jedoch durchaus begünstigt werden. Die diesbezüglichen Grundvoraussetzungen sind eine stabile Entwicklung der Inlandsnachfrage und eine geringe Exportabhängigkeit des Handwerks. Ist die Konjunktursituation aufgrund einer schwachen Binnennachfrage ungünstig, kann das Handwerk aber auch als Krisenverstärker wirken. Vor allem die konjunkturellen Bewegungen im Bausektor und in abgeschwächter Form diejenigen im Konsumgüterbereich bestimmen dabei, ob das Handwerk stabilisiert oder nicht.

2. Da sich an der geringen Exportabhängigkeit des Handwerks im Wesentlichen in Zukunft nichts ändern dürfte, wird die Handwerkswirtschaft aller Voraussicht nach auch künftig von exportinduzierten Wirtschaftskrisen - wie am aktuellen Beispiel gezeigt - weniger stark betroffen sein als andere Wirtschaftsbereiche und in solchen Fällen damit eher als Stabilisator wirken.

3. Ein relativ beharrendes Beschäftigungsverhalten eines Großteils der Handwerkswirtschaft spricht dafür, dass das Handwerk im Bereich des Arbeitsmarkts eine stabilisierende Funktion besitzt.

Mit den Ergebnissen des vorliegenden Beitrags sind verschiedene wirtschaftspolitische Implikationen verbunden:

- Das Handwerk kann nur dann als Konjunkturstabilisator wirken, wenn sich die Inlandsnachfrage entsprechend stabil entwickelt. Dies zeigt, wie wichtig aus Sicht des Handwerks konkrete Maßnahmen zur Stärkung des Binnenmarkts sind. 
- Gleichwohl dürfte die deutsche Wirtschaft weiterhin nicht umhin können, auf die Exportund Weltmarktorientierung als wesentlichen Wachstumstreiber zu setzen. Eine etwaige Stabilisatorfunktion des Handwerks dürfte daher auch zukünftig bedeuten, dass die Handwerkswirtschaft von wichtigen Wachstumsbereichen der deutschen Wirtschaft weitgehend abgekoppelt bleibt. In den Betrieben und Organisationen des Handwerks sollten daher die Wogen der Weltkonjunktur stärker als bisher als lohnenswerte Chance begriffen werden. Dies kann zum einen durch eine unmittelbare Ausweitung der handwerklichen Auslandsgeschäfte geschehen. ${ }^{86}$ Hinsichtlich der indirekten Exportimpulse wäre zum anderen eine stärkere Einbindung des Handwerks in die gesamtwirtschaftliche Wertschöpfungskette wichtig.

- Das beharrende Beschäftigungsverhalten eines Großteils des Handwerks zeigt, dass die Handwerkswirtschaft eine wichtige konjunkturelle Stabilisatorfunktion im Bereich des Arbeitsmarkts einnimmt. Bei Bedarf sollten daher die Handwerksbetriebe durch geeignete wirtschaftspolitische Maßnahmen in die Lage versetzt werden, ihre Beschäftigten auch in konjunkturell schwierigen Zeiten möglichst lange zu halten. ${ }^{87}$ Darüber hinaus setzt die Erfüllung einer solchen Funktion die Sicherstellung des Personalangebots voraus. Damit das Handwerk daher zukünftig in Zeiten des verschärften Fachkräftemangels eine beschäftigungsstabilisierende Funktion ausüben kann, sind entsprechende Anstrengungen seitens der Betriebe, der Handwerksorganisationen und der Politik gefragt.

Mit Blick auf künftige Forschungsarbeiten ist eine Differenzierung der vorgelegten Ergebnisse wünschenswert. Hierzu wäre eine Auswertung der Handwerksberichterstattung nach Unternehmensgrößenklassen von besonderer Relevanz. Die gestiegene Bedeutung von flexiblen Formen der Beschäftigung im Handwerk (z.B. Teilzeitbeschäftigte oder befristete Arbeitsverhältnisse) konnten im Rahmen der vorliegenden Untersuchung ebenfalls nicht berücksichtigt werden. Zur Bekräftigung der gewonnenen Ergebnisse wäre es ferner von Interesse, die nur für das zulassungspflichtige Handwerk vorgenommene Analyse mit entsprechenden Daten auf das Gesamthandwerk ausdehnen zu können.

\section{Literaturverzeichnis}

Abelshauser, W. (2004): Deutsche Wirtschaftsgeschichte seit 1945, Lizenzausgabe der Bundeszentrale für Politische Bildung, Bonn.

Beckermann, T. (1974): Das Handwerk im Wachstum der Wirtschaft. Eine statistische Analyse, Schriftenreihe des Rheinisch-Westfälischen Instituts für Wirtschaftsforschung Essen, Heft 34, Berlin.

86 Wie eine Untersuchung des ifh Göttingen zeigt, besteht in der Handwerkswirtschaft noch ein erhebliches Exportpotenzial. Vgl. Müller, K. (2008), S. 33 ff und S. 63 f.

87 Die erleichterte Inanspruchnahme des Kurzarbeitergeldes im Rahmen des zweiten Konjunkturpakets kann z.B. als entsprechende Maßnahme gewertet werden. 
Burghardt, M. (2006): Zur Revision der privaten Konsumausgaben im Rahmen der Volkswirtschaftlichen Gesamtrechnungen 2005, in: Wirtschaft und Statistik, Statistisches Bundesamt, Heft 2/2006, S. 136-144.

Creditreform (2003): Wirtschaftslage Handwerk. Frühjahr 2003, Neuss.

Creditreform (2009): Wirtschaftslage Handwerk. Frühjahr 2009, Neuss.

Dispan, J. (2003): Regionale Strukturen und Beschäftigungsperspektiven im Handwerk: Regionalanalyse, Entwicklungstrends, Herausforderungen, regionalpolitische Handlungsfelder, Umsetzungsansätze in der Region Stuttgart, IMU-Informationsdienst, Heft 4/03, München.

Duong, M. H. (2002): Abgebrochener Aufschwung belastet Investitionstätigkeit, in: Wochenbericht des DIW Berlin, Heft 38/2002, S. 638-642.

Dürig, W., Lagemann, B., Rothgang, M., Trettin, L. und Welter, F. (2004): Determinanten des Strukturwandels im deutschen Handwerk. Band 1, Essen.

Horn, G., Sturn, S. und Treeck, T. (2010): Die Debatte um die deutsche Exportorientierung, in: Wirtschaftsdienst. Zeitschrift für Wirtschaftspolitik, Jg. 90, Heft 1, S. 22-28.

ifo (2003): Gemeinschaftsdiagnose. Die Lage der Weltwirtschaft und der deutschen Wirtschaft im Frühjahr 2003, in: ifo Schnelldienst, Jg. 56, Heft 7/2003.

ifo (2004): Gemeinschaftsdiagnose. Die Lage der Weltwirtschaft und der deutschen Wirtschaft im Herbst 2004, in: ifo Schnelldienst, Jg. 57, Heft 20/2004.

ifo (2005): Gemeinschaftsdiagnose. Die Lage der Weltwirtschaft und der deutschen Wirtschaft im Herbst 2005 in: ifo Schnelldienst, Jg. 58, Heft 20/2005.

ifo (2006): Gemeinschaftsdiagnose. Die Lage der Weltwirtschaft und der deutschen Wirtschaft im Herbst 2006, in: ifo Schnelldienst, Jg. 59, Heft 20/2006.

ifo (2007): Gemeinschaftsdiagnose. Die Lage der Weltwirtschaft und der deutschen Wirtschaft im Frühjahr 2007, in: ifo Schnelldienst, Jg. 60, Heft 8/2007.

ifo (2008): Folgen der US-Immobilienkrise belasten die Konjunktur. Gemeinschaftsdiagnose Frühjahr 2008, in: ifo Schnelldienst, Jg. 61, Heft 8/2008.

ifo (2009a): Im Sog der Weltrezession. Gemeinschaftsdiagnose Frühjahr 2009, in: ifo Schnelldienst, Jg. 62, Heft 8/2009.

ifo (2009b): Zögerliche Belebung - steigende Staatsschulden. Gemeinschaftsdiagnose Herbst 2009, in: ifo Schnelldienst, Jg. 62, Heft 20/2009.

ifo (2010): Erholung setzt sich fort - Risiken bleiben groß. Gemeinschaftsdiagnose Frühjahr 2010, in: ifo Schnelldienst, Jg. 63, Heft 08/2010.

IW-Köln (2009): In der Tiefebene - IW-Konjunkturprognose Frühjahr 2009, Institut der deutschen Wirtschaft Köln, in: IW-Trends, Heft 2/2009, S. 15-47.

IW-Köln (2010): Handwerk - Fast ohne Schrammen durch die Krise, in: Informationsdienst des Instituts der deutschen Wirtschaft Köln, Jg. 36, Heft 17, S. 4-5.

KfW, Creditreform, IfM, ZEW und DtA (2003): MittelstandsMonitor 2003 - Jährlicher Bericht zu Konjunktur- und Strukturfragen kleiner und mittlerer Unternehmen, Frankfurt am Main. 
KfW, Creditreform, IfM, RWI und ZEW (2010): Konjunkturelle Stabilisierung im Mittelstand - aber viele Belastungsfaktoren bleiben, MittelstandsMonitor 2010 - Jährlicher Bericht zu Konjunktur- und Strukturfragen kleiner und mittlerer Unternehmen, Frankfurt am Main.

Kornhardt, U. und Kucera, G. (2003): Investitionsverhalten im Handwerk: Ursachen für die Investitionsschwäche im Handwerk seit Mitte der 90er Jahre, Göttinger handwerkswirtschaftliche Studien, Nr. 68, Duderstadt.

Lageman, B., Dürig, W. und Trettin, L. (2005): Determinanten des Strukturwandels im Handwerk, in: Bundesarbeitsblatt, Heft 7-2005, S. 4-16.

Lehmann, S. und Müller, K. (2010): Cluster im Handwerk. Eine Analyse hinsichtlich deren Vorkommen und Bedeutung, Göttinger Handwerkswirtschaftliche Studien, Nr. 80, Duderstadt.

Loschky, A. und Ritter, L. (2007): Konjunkturmotor Export, in: Wirtschaft und Statistik, Statistisches Bundesamt, Heft 5/2007, S. 478-488.

Maußner, A. (1994): Konjunkturtheorie, Berlin.

Momm, H.-J. (1983): Konjunkturprognosen im Handwerk. Systeme der Früherkennung von Konjunkturbetroffenheiten in ausgewählten Handwerkszweigen, Göttinger handwerkswirtschaftliche Studien, Nr. 32, Göttingen.

Müller, K. (2005): Beschäftigung im Handwerk, Göttinger Handwerkswirtschaftliche Studien, Nr. 72, Duderstadt.

Müller, K. (2008): Auslandsgeschäfte im Handwerk, Göttinger Handwerkswirtschaftliche Studien, Nr. 76, Duderstadt.

Müller, K. (2010): Statistische Datenquellen für das Handwerk, Göttinger Handwerkswirtschaftliche Studien, Nr. 81, Duderstadt.

Müller, K., Koschmieder, K.-D., Trombska, D., Zapfe, A. und Rötzler, K. (2009): Unternehmensnachfolge im Thüringer Handwerk. Eine Analyse im Zeichen des demografischen Wandels, Göttinger Handwerkswirtschaftliche Studien, Nr. 78, Duderstadt.

Neuhäuser, J. (2008): Verwaltungsdaten ersetzen Konjunkturerhebungen im Handwerk, in: Wirtschaft und Statistik, Statistisches Bundesamt, Heft 5/2008, S. 398-408.

Pätzold, J. und Baade, D. (2008): Stabilisierungspolitik, 7 Aufl., München.

PKM (2009): Die Konjunkturpakete der Bundesregierung aus Sicht des Handwerks, in: PKM Journal, Parlamentskreis Mittelstand der CDU/CSU-Bundestagsfraktion, Sonderausgabe Handwerkspolitik, Heft III/2009, S. 16.

Roos, M. W. M. (2007): Die makroökonomischen Wirkungen diskretionärer Fiskalpolitik in Deutschland - Was wissen wir empirisch?, in: Perspektiven der Wirtschaftspolitik, Jg. 8, Heft 4, S. 293-308.

Roos, M. W. M. (2009): Die deutsche Fiskalpolitik während der Wirtschaftskrise 2008/2009, in: Perspektiven der Wirtschaftspolitik, Jg. 10, Heft 4, S. 389-412.

Sachverständigenrat (2009): "Die Zukunft nicht aufs Spiel setzen", Jahresgutachten 2009/10 des Sachverständigenrats zur Begutachtung der gesamtwirtschaftlichen Entwicklung, in: http://www.sachverstaendigenrat-wirtschaft.de/download/gutachten/ga09_ges.pdf (letzter Zugriff: 30.03.2010). 
Schirwitz, B., Seiler, C. und Wohlrabe, K. (2008): Regionale Konjunkturzyklen in Deutschland - Teil I: Die Datenlage, in: ifo Schnelldienst, Jg. 62, Heft 13.

Schmidt, K.-H. (1975): Die Konjunkturabhängigkeit des Handwerks, in: Aßmann, K. und Schmidt, K.-H. (Hrsg.), Die Konjunkturabhängigkeit der Klein- und Mittelbetriebe, Göttinger handwerkswirtschaftliche Studien, Nr. 26, Göttingen, S. 1-171.

Speth, H.-T. (2004): Komponentenzerlegung und Saisonbereinigung ökonomischer Zeitreihen mit dem Verfahren BV4.1, in: Methodenberichte, Statistisches Bundesamt, Heft 3.

Statistisches Bundesamt (2009): Statistische Monatszahlen, in: Wirtschaft und Statistik, Statistisches Bundesamt, Heft 10/2009.

Statistisches Bundesamt (2010): Volkswirtschaftliche Gesamtrechnungen. Inlandsproduktsberechnung, Detaillierte Jahresergebnisse 2009, in: Statistisches Bundesamt, Fachserie 18, Reihe 1.4.

Steinfelder, J. (2007): Exportweltmeister Deutschland - Titel auf Zeit? Der deutsche Außenhandel 2006 und seine Märkte, in: Wirtschaft und Statistik, Statistisches Bundesamt, Heft 4/2007, S. 362-369.

Tichy, G. (1994): Konjunktur. Stilisierte Fakten, Theorie, Prognose, 2. Aufl., Berlin.

Winker, P. (2007): Empirische Wirtschaftsforschung und Ökonometrie, 2. Aufl., Berlin.

ZDB (2007): Baumarkt 2006 - Ergebnisse, Entwicklungen, Tendenzen, Zentralverband des Deutschen Baugewerbes (ZDB), Berlin.

ZDB (2009): Baumarkt 2008 - Ergebnisse, Entwicklungen, Tendenzen, Zentralverband des Deutschen Baugewerbes (ZDB), Berlin.

ZDH (2009a): Konjunkturbericht 1/2009, Kein Einbruch der Handwerkskonjunktur - Aber starke Verlangsamung und gedämpfte Erwartungen, Zentralverband des Deutschen Handwerks (ZDH), Berlin.

ZDH (2009b): Konjunkturbericht 2/2009, Handwerkskonjunktur spürbar belebt - Starke Unterschiede zwischen den Branchen, Zentralverband des Deutschen Handwerks (ZDH), Berlin.

ZDH (2009c): Kurzbericht Konjunktur 4. Quartal 2009, Handwerkskonjunktur stabilisiert sich weiter - bei großen Gruppenunterschieden, Zentralverband des Deutschen Handwerks (ZDH), in: http://www.zdh.de (letzter Zugriff: 27.04.2010).

ZDK (2009): Zahlen \& Fakten 2008, Zentralverband des deutschen Kraftfahrzeuggewerbes (ZDK), Bonn. 